\title{
CHALMERS
}

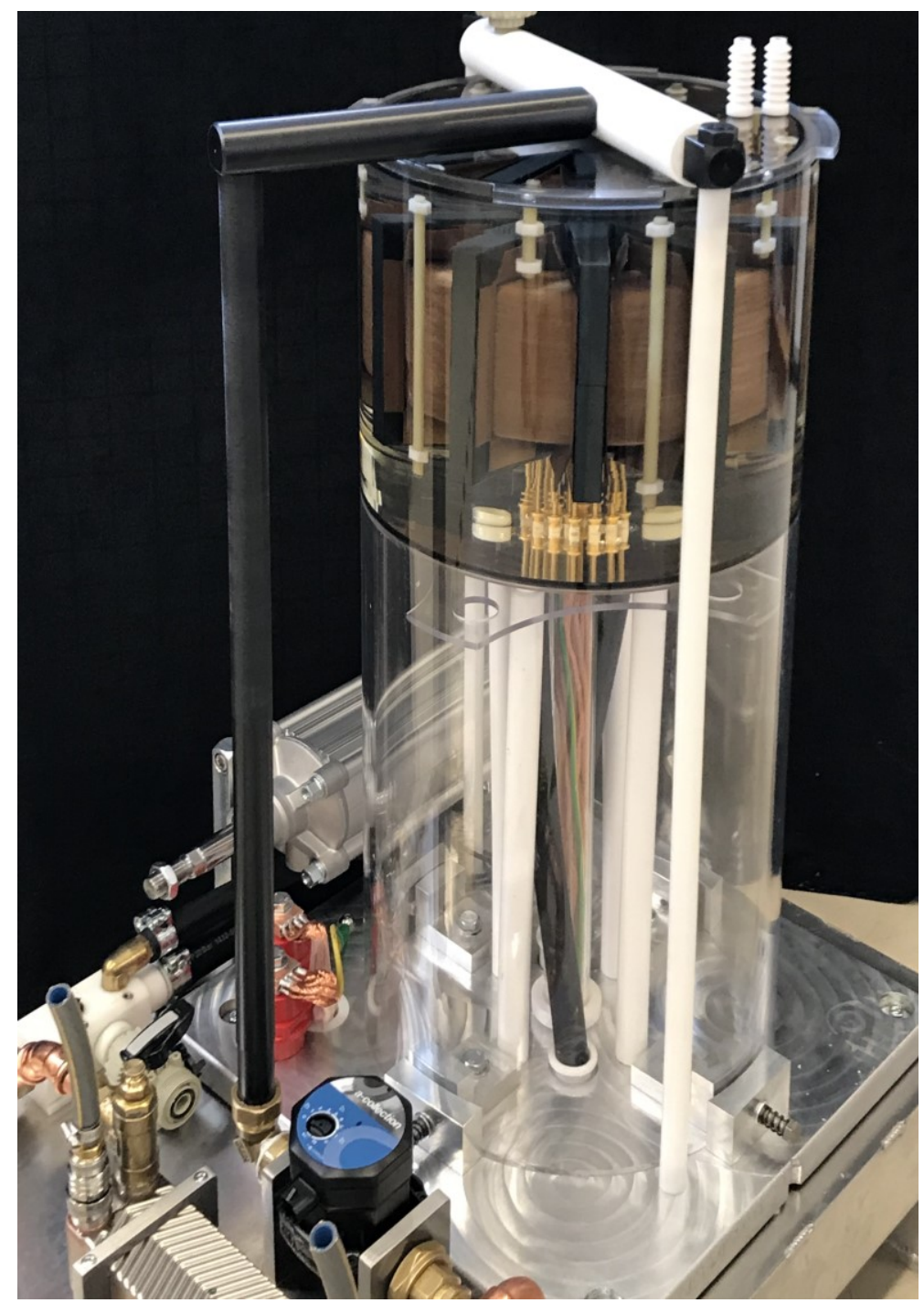

\section{A Novel Oil-immersed Medium Frequency Transformer for Offshore HVDC Wind Farms}

MOHAMMAD KHAREZY

Department of Electrical Engineering

Chalmers University of Technology

Gothenburg, Sweden 2020 
THESIS FOR THE DEGREE OF LICENTIATE OF ENGINEERING

A Novel Oil-immersed Medium Frequency Transformer for Offshore HVDC Wind Farms

MOHAMMAD KHAREZY

Department of ELECTRICAL ENGINEERING

CHALMERS UNIVERSITY OF TECHNOLOGY

Gothenburg, Sweden 2020 
A Novel Oil-immersed Medium Frequency Transformer for Offshore HVDC Wind Farms

\section{MOHAMMAD KHAREZY}

(C) MOHAMMAD KHAREZY, 2020.

Department of Electrical Engineering

Chalmers University of Technology

SE-412 96 Gothenburg

Sweden

Telephone $+46(0) 31-7721000$

Cover:

The Designed, Manufactured and Tested Novel Prototype Oil-immersed 125 kV HVDC Insulated 5 kHz Medium Frequency $0.42 \mathrm{kV} / 5 \mathrm{kV}$ Transformer.

Chalmers Library, Report Service

Gothenburg, Sweden 2020 
Har du en dröm,

fär du jaga den.

om drömmen är paj,

fär du laga den.

Har du ingen dröm,

fär du skapa en

och sätta stop för dem

som vill skada den.

så làt dem títta på díg.

ja! de kan döma díg.

Fortsätt vara du!

Jag lovar att det lönar sig.

vem e som oss

Song by Anis Don Demina 


\section{Abstract}

In this project, a design of an oil type medium frequency transformer for offshore wind farm applications is proposed. The design is intended for applications when series coupling of the output of the DC/DC converters of the wind turbine on their secondary side is done to achieve a costeffective high voltage solution for collecting energy from offshore wind parks. The focus of the work is on the insulation design of the high voltage side of the medium frequency transformer where the magnetic design constraints should also be satisfied.

Above all, a proof of concept is made demonstrating a possible solution for the design of the transformer for such a DC/DC converter unit. The transformer suggested is using oil/paper as insulation medium. Furthermore, characterisation of an eco-friendly biodegradable transformer oil for this type of HVDC transformer application is made. Moreover, an introduction of reliable high frequency characterisation test methods to medium frequency transformer designers is made. In addition, the Non-Linear Maxwell Wagner (NLMW) relations are further developed to form a method for the development of an HVDC MFT transformer. All in all, the DC series concept has been further developed one step closer to pre-commercialization, i.e. from TRL 1 to about 2. 


\section{Acknowledgment}

This work has been carried out at RISE Research Institutes of Sweden and the Department of Electrical Engineering at Chalmers University of Technology. The financial support was given by Swedish Energy Agency (Energimyndigheten) 44983-1, as part of the research program "Forskning och innovation inom vindkraftsområdet (VindEL) 2017".

First, I express my sincere thanks to my examiner and main supervisor Prof. Torbjörn Thiringer, without his help the initiation and realization of this project would be impossible. I would also thank my former supervisor Dr. Mohammad Amin Bahmani for his kind support with the application to funding and the knowledge transferred to me related this project. My supervisor from RISE, Dr. PerOlof Hedekvist and co-supervisors from Chalmers, Prof. Yuriy Serdyuk and Dr. Thomas Hammarström are gratefully acknowledged for their continuous support.

While conducting this project, I had the warm and unconditional support of my friends Dr. Morteza Eslamian and Dr. Hassareza Mirzaei, Babak Alikhanzadeh and Behrouz Bayat. I thank them wholeheartedly.

I acknowledge the effective contribution of the reference group members, Dr. Seyed Ali Mousavi from ABB power grid research center in Sweden, Prof. Sonja Lundmark from Chalmers and Charlotte Bergqvist from Trinda Energy $A B$.

Many thanks go to my Master and Bachelor thesis students Oriol Guillén Sentís, Joakim Rastamo, and Marcus Svensson who helped me effectively and with all their best to cover all I promised to do.

Special thanks go to Dr. Seyed Ali Mousavi from ABB power grid research center in Sweden for the core loss measurements, Kim Bolleter from Weidmann Electrical Technology AG and Espen Doedens from Nexsans Subsea Energy Systems for providing insulation material samples and Harri Järvinen from Habia Cable for providing a High Voltage Cable sample for free and again to Energimyndigheten for sponsoring the rest of the materials needed in the thesis work. I should also thank Dr. Xiangdong $\mathrm{Xu}$ for his effective help for buying formed insulation parts from Taizhou Xinyuan Electrical Equipment Co. Ltd and his guidelines for conductivity measurements.

I need also to thank all my colleagues at RISE and Chalmers whose warm technical help was invaluable; among them, my former colleague Johan Söderbom, Dr. Anders Bergman, Tatu Nieminen, Andreas Nilsson, Andreas Andersson, Karl-Erik Rydler, Mathias Nordlund, Stefan Fors, Reine Weijmer, Dr. Alf-Peter Elg, Fredrik Arrhén, Tanja Tränkle and Petra Lantz.

Eventually, my heartfelt gratitude goes to my family for all their patience, support and love.

Mohammad Kharezy

Gothenburg, Sweden, 2020 


\section{List of Abbreviations}

DC, Direct Current

AC, Alternating current

HV, High Voltage

LV, Low Voltage

MFT, Medium Frequency Transformer

DAB, Dual Active Bridge

ZVS, Zero Voltage Switching

PWM, Pulse Width Modulator

PD, Partial Discharge

BDV, Breakdown Voltage

OIP, Oil Impregnated Pressboard 


\section{Contents}

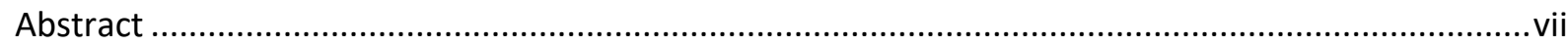

Acknowledgment...................................................................................................................

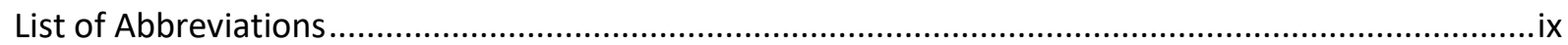

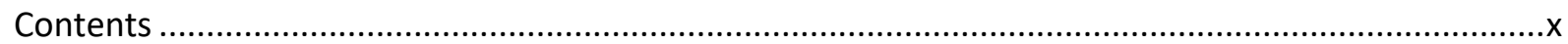

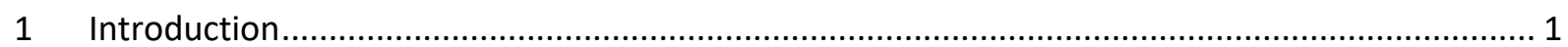

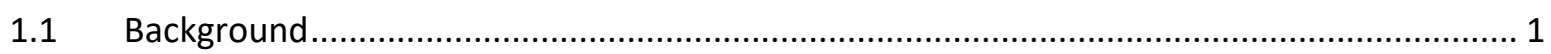

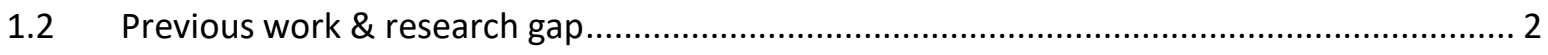

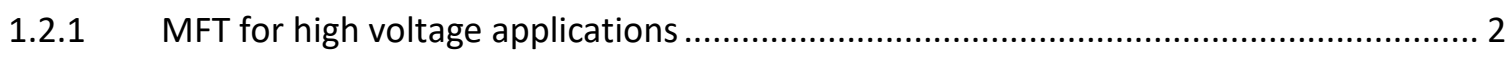

1.2.2 High voltage insulation under HVDC stress .............................................................. 4

1.2.3 Importance of measurement of electrical conductivity .............................................. 5

1.2.4 High voltage insulation under high frequency stress .............................................. 6

1.2.5 Importance of determining leakage inductance of MFTs ............................................ 7

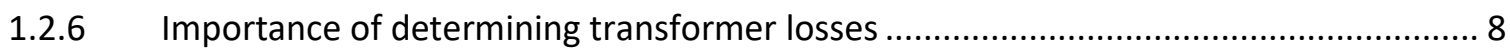

1.3 Required information on oil type MFT's components .................................................... 9

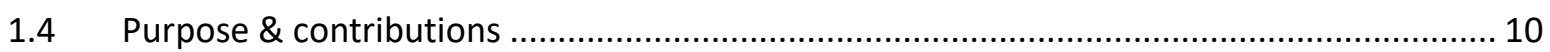

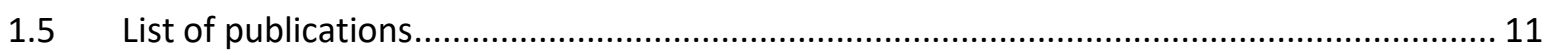

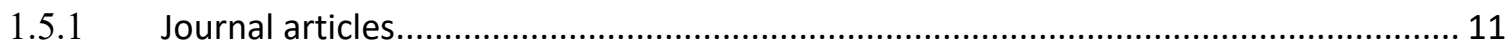

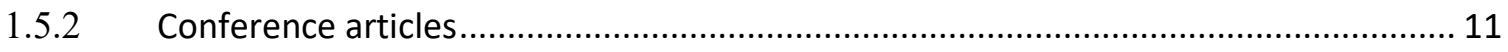

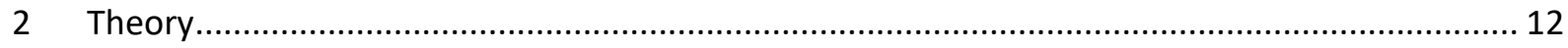

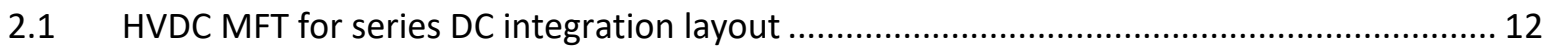

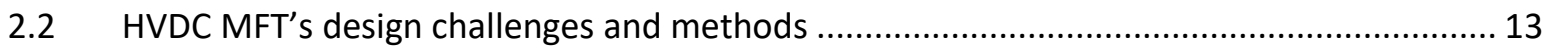

2.2.1 Non-suitability of dry insulations for high voltage DC transformers............................ 13

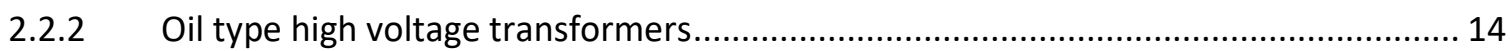

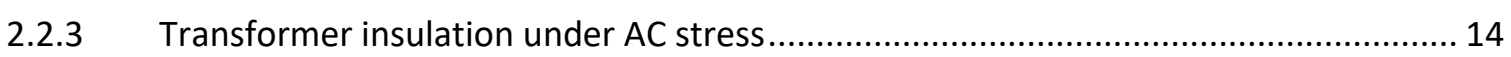

2.2.4 Effect of solid barriers at oil type transformers ...................................................... 15

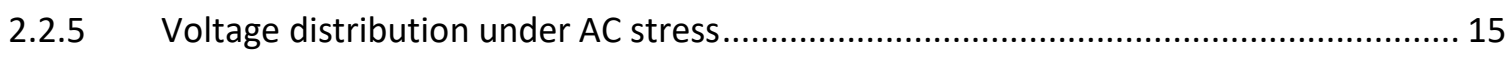

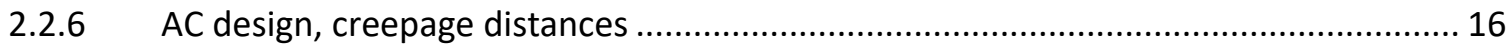

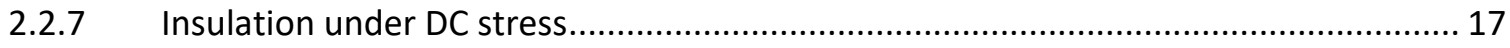

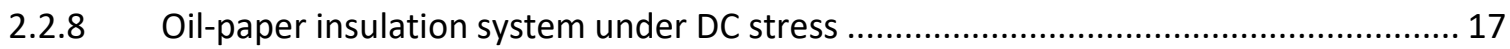

2.2.9 Effect of unequal conductivities on varying voltage distribution under DC stress ....... 19

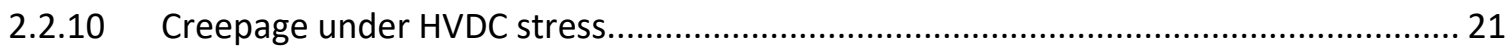

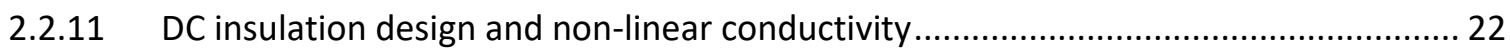




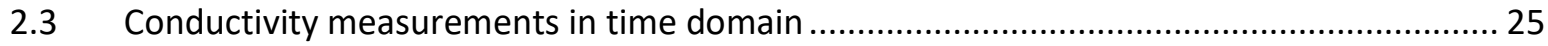

2.3.1 Conductivity measurement system set-up.................................................................. 26

2.3.2 High voltage conductivity measurement test cell ...................................................... 26

2.3.3 OIP conductivity values calculation using charge difference method ........................... 27

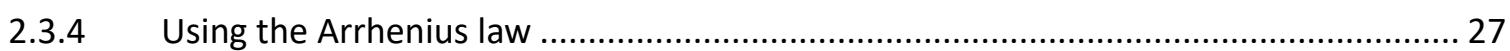

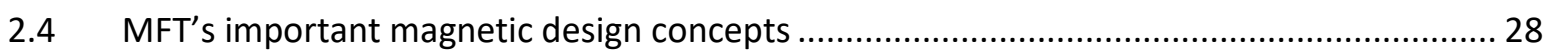

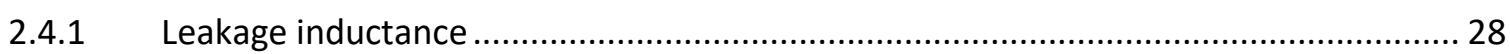

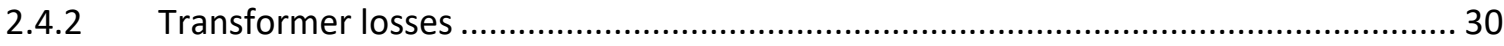

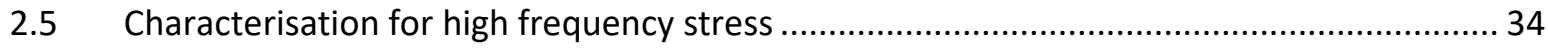

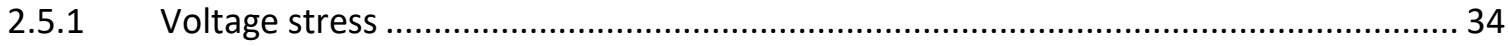

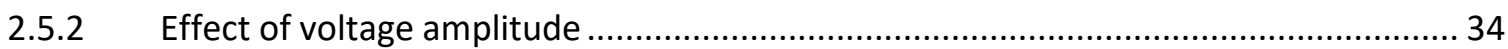

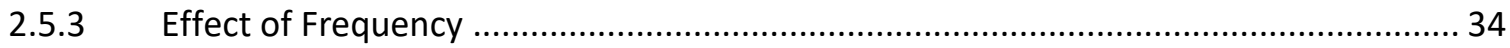

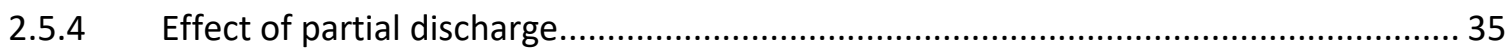

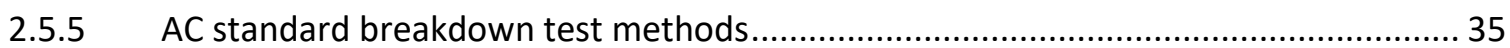

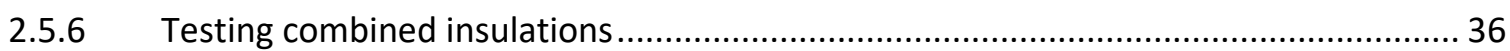

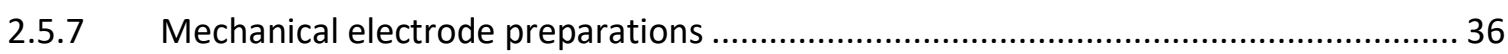

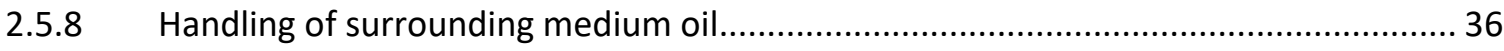

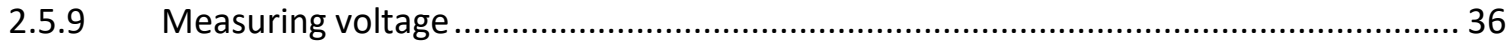

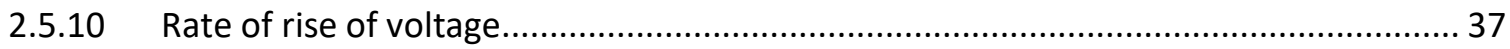

2.5.11 Monitoring PD during an aging test or before an ACBD test ..................................... 37

3 Characterisation of insulation material for HVDC MFT application ........................................... 38

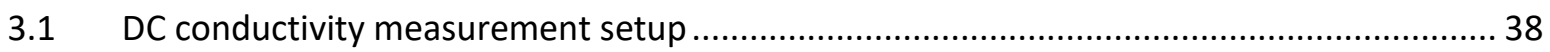

3.1.1 Current measuring and data acquisition system and its protection .............................. 40

3.1.2 High voltage application system and safety .............................................................. 41

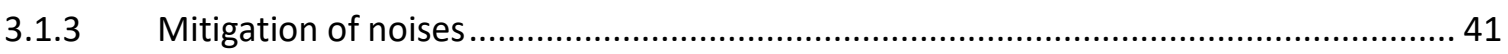

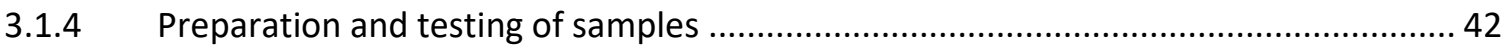

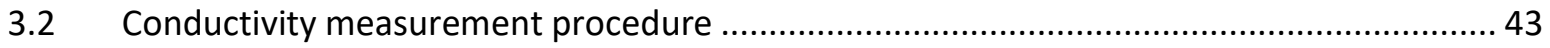

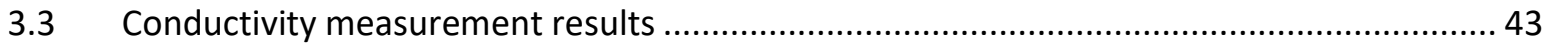

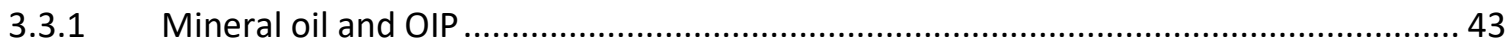

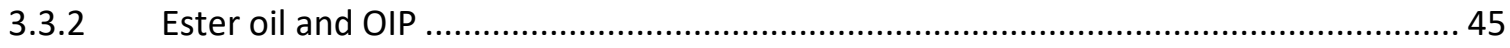

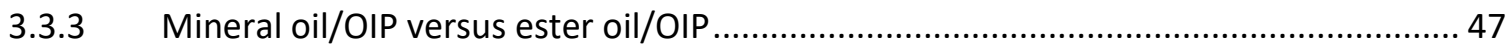

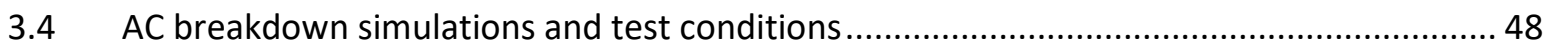

3.4.1 Effect of shape of electrodes and surrounding medium to the results of the tests ..... 48

3.5 High frequency withstand tests on the Litz wire ............................................................. 49 
3.5.1 Withstand test setup used for the project ............................................................. 49

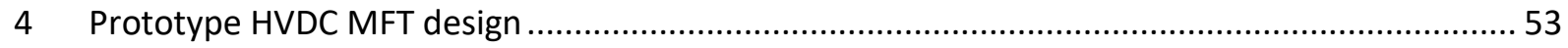

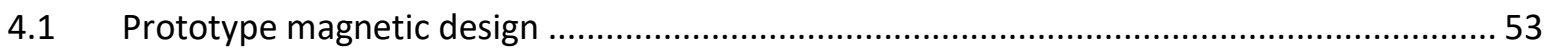

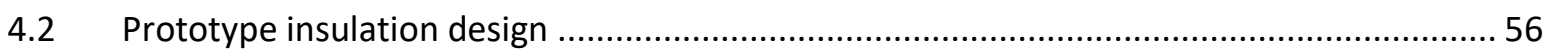

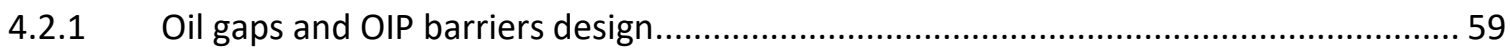

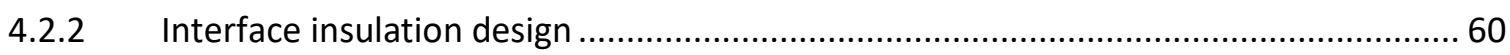

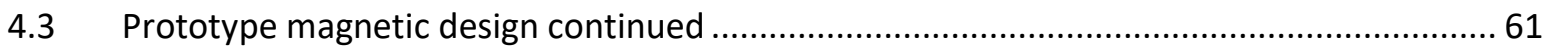

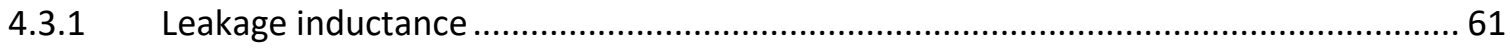

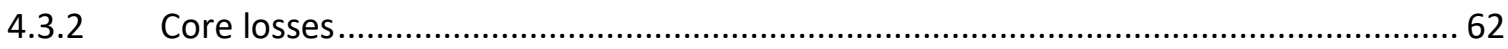

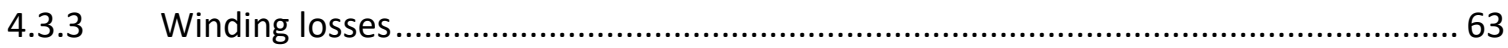

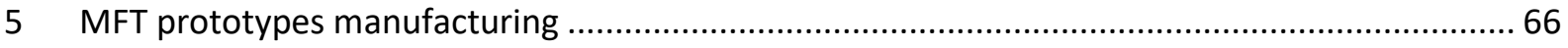

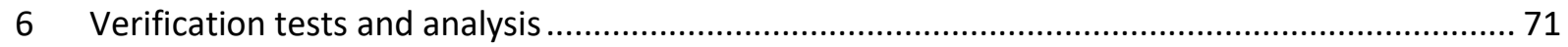

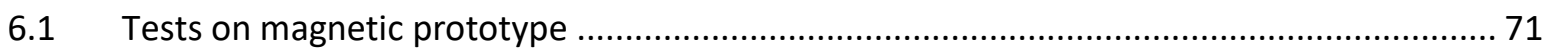

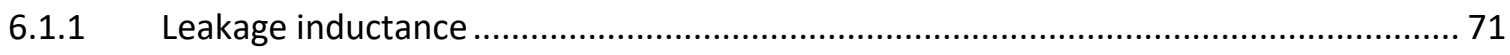

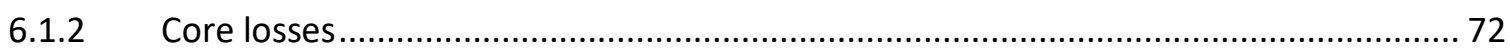

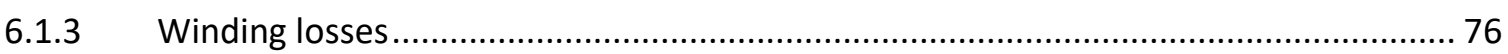

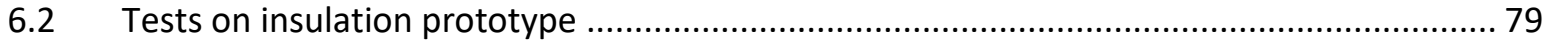

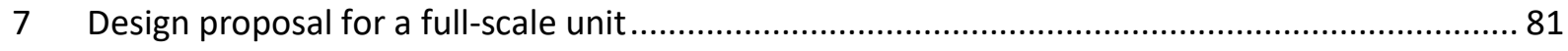

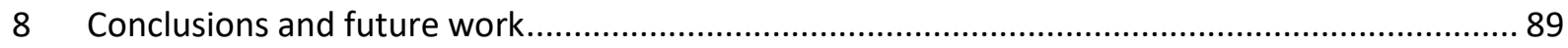

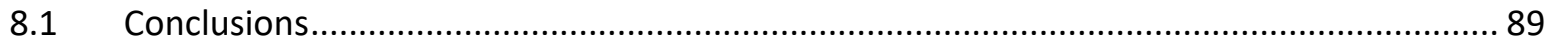

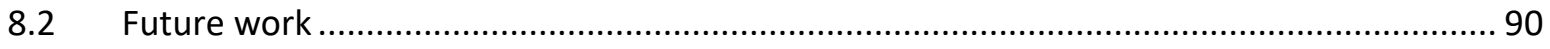

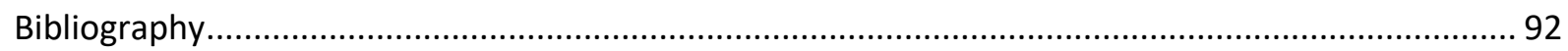




\section{Introduction}

\subsection{Background}

Offshore wind power has an enormous potential for global electricity production according to the International Energy Agency IEA [1]. It is a potential that needs to be exploited to counteract the trend of rising $\mathrm{CO} 2$ emissions from electricity generation worldwide. IEA estimates that over $120 \mathrm{GW}$ of offshore wind power will be available in Europe by 2040. With a capacity factor that generally is better than that of onshore wind power stations and better than solar power, offshore wind contributes to the security of supply and a stable electricity system. In addition to having stable policy frameworks and the ever-increasing size and number of wind turbines, continued technology development is needed in all parts of the wind energy chain to reduce costs. There is an extensive installation of wind energy parks taking place in the North Sea, as well as at other offshore areas. The main reason is that wind condition is better at sea as well as the fact that it is becoming more and more difficult to find locations on land where wind turbines can be installed without causing environmental disturbances.

Today, when a sea-based wind park reaches a size of some hundreds of MWs or more, a platform (or several ones), acting as the hub in the collection grid and a point of connection for the cable to land is needed. Among other equipment the platform carries a transformer (typically 30/230 kV), an example is the Lillgrund (110 MW) wind park, south of the Öresund bridge. Since ordinary AC cables becomes inefficient over approximately $100 \mathrm{~km}$ [2], it has become necessary to move over to DCtransmission for some of the new wind parks, an example is the Borwind wind park north-west of the German North sea coast. However, within the wind park, still a collection grid running at $50 \mathrm{~Hz} A C$ is used and two platforms of gigantic sizes are needed for $50 \mathrm{~Hz} \mathrm{AC/AC}$ and AC/DC conversion increasing the cost substantially for the wind energy installation.

A possibility here is to use the DC-technology also for the energy collection grid within the wind park. To do that, a key component is missing, the high-power DC/DC converter, that can produce a DC output voltage from the wind turbines of sufficient voltage level. Such a device can transform the voltage from the wind turbine to a high DC voltage using a much smaller converter unit compared to a $50 \mathrm{~Hz}$ transformer. An idea is to fit the converter system into a container on the outside of a wind turbine, thus utilizing the existing foundation out in the sea (See Figure 1-1); however, a location inside the tower or nacelle is also an option.

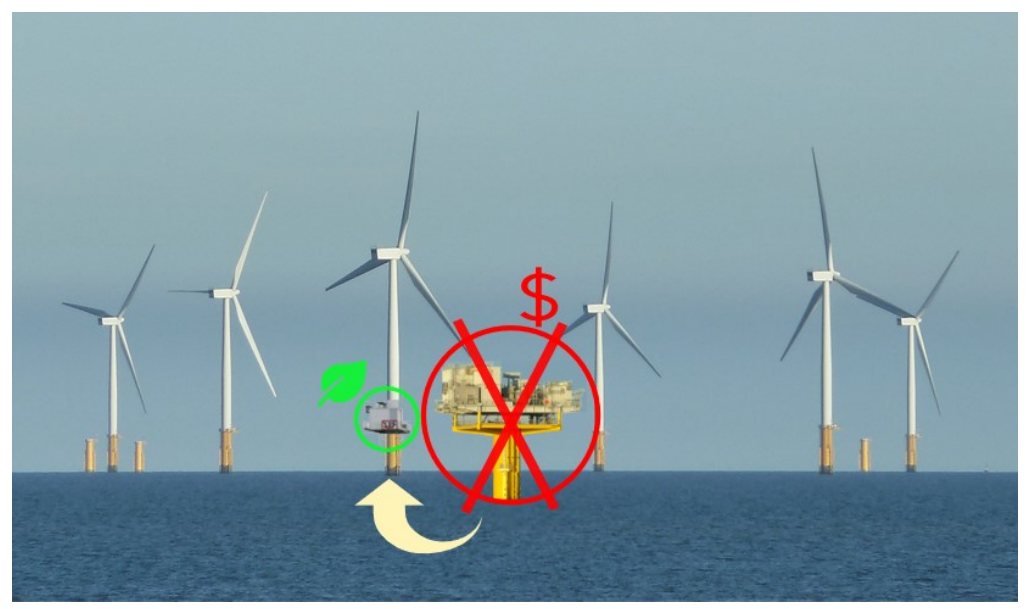

Figure 1-1: Wind turbine- mounted DC/DC converter 
A highly interesting solution is then to connect the output of the wind turbines in series, and in this way making the voltage level to reach 100 or even $200 \mathrm{kV}$. The idea would then be to continue the connection directly to shore without the need of a large transformer platform. A cable can transport up to $2000 \mathrm{~A}$, and using a bipolar set-up, $800 \mathrm{MW}$ can be reached without a platform, for the 200kV case. This is a huge investment saving. However, here comes a highly important factor: The wind turbine that is located closest to the DC-transmission cables going away to shore must take up the full insulation on its high-voltage side, and the duty falls on the output DC/DC converter. Today, the DC/DC converter technology is far away from such capabilities. This is where the proposed project comes in.

The proposed design approach that will be developed within this thesis, was introduced earlier in a Ph.D. work [3] where it was applied on two down-scaled $50 \mathrm{~kW}, 1 / 3 \mathrm{kV}, 5 \mathrm{kHz}$ prototype MediumFrequency Transformers (MFTs). These designs have later been manufactured (in a joint effort between Chalmers and RISE), and successfully tested, fulfilling the efficiency, power density and leakage inductance requirements that the prototypes were designed for [4]. The efficiency has been verified experimentally to be $99.67 \%$ and a power density of $12.2 \mathrm{~kW} / \mathrm{I}$ which is nearly 13 times more than a normal $50 \mathrm{~Hz}$ transformer, was found.

\subsection{Previous work \& research gap}

\subsubsection{MFT for high voltage applications}

Heinemann et al. (ABB) in their work published in 2002 [5] have presented a design for a shell type coaxial windings $350 \mathrm{~kW}, 3 / 3 \mathrm{kV}, 10 \mathrm{kHz}$ prototype transformer. The transformer is designed to be used in a multilevel converter and be coupled to a $15 \mathrm{kV}, 162 / 3 \mathrm{~Hz}$ line. A $95 \mathrm{kV}$ Lightning Impulse (LI) test is also performed. This transformer is not claimed to withstand HVDC voltage to ground and moreover, at $A C 50 \mathrm{~Hz}$, and its PD-free insulation level is $28 \mathrm{kV}$ to ground for a one-minute application of voltage. This is an example of the highest voltage that a dry type MFT can deliver for a PD-free application.

Steiner et al. (Bombardier) in their work published in 2007 [6] have presented a design for a core type and hallow conductor windings $350 \mathrm{~kW}, 1 / 1 \mathrm{kV}, 8 \mathrm{kHz}$ prototype transformer. The transformer is designed to be used in a multilevel converter and be coupled to a $15 \mathrm{kV}, 162 / 3 \mathrm{~Hz}$ line. A $100 \mathrm{kV}$ Lightning Impulse (LI) test is also performed. The high voltage DC insulation to ground is not investigated and even at the AC $50 \mathrm{~Hz}$, its PD-free insulation level is $33 \mathrm{kV}$. This design also has the same PD inception level as the previous dry type transformer.

STS Transformers in 2015 has presented a design for a core type cast resin windings $450 \mathrm{~kW}, 1.8 / 1.8$ $\mathrm{kV}, 8 \mathrm{kHz}$ prototype transformer. The transformer is designed to be used in a multilevel converter and be coupled to a $15 \mathrm{kV} @ 16$ 2/3 Hz \& $25 \mathrm{kV} @ 50 \mathrm{~Hz}$. At AC $50 \mathrm{~Hz}$ its PD-free insulation level is 37 $\mathrm{kV}$ to ground [7]. However, no information regarding the performance of the transformer under HVDC stress is presented.

Bahmani (Chalmers) in his work published has in 2016 [9] has presented a design for a shell type coaxial windings $666 \mathrm{~kW}, 1 / 2 \mathrm{kV}$ transformer as one stage of 15 series connected DC/DC converters. The total rating of the turbine is $10 \mathrm{MW}$, the turbine's output voltage is $1 \mathrm{kV}$ and the output voltage of the farm is $30 \mathrm{kV}$ (see Figure 2-2). The insulation level of the high voltage side to ground is designed to be $60 \mathrm{kV}$. However, the insulation design is only based on a $\mathrm{kV} / \mathrm{mm}$ high voltage strength figures of the insulation materials used as coil-former and a safety factor of 2 . He has also designed 
and manufactured a $50 \mathrm{~kW}, 1 / 3 \mathrm{kV}, 5 \mathrm{kHz}$ prototype transformer which has not been designed or proved to withstand a high DC voltage to ground which is supposed to be applied to the winding in a series DC wind farm configuration. Other parameters like insulation design for a combined insulation systems and non-uniform electric fields were not included in the scope of the study.

Figure 1-2 presents the manufactured prototypes.

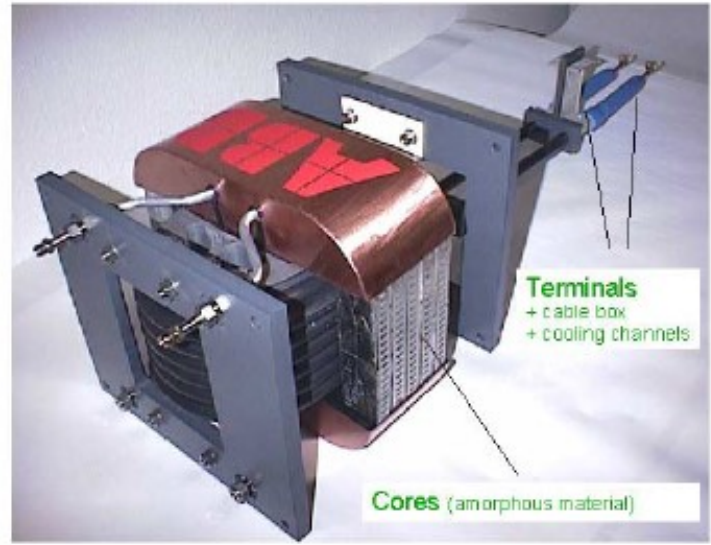

$350 \mathrm{~kW}, 3 / 3 \mathrm{kV}, 10 \mathrm{kHz}$

Heinemann et al. (ABB), 2002 [5]

Claimed HV insulation to ground 15 kV @16 2/3 Hz $P D$ free $A C 50 \mathrm{~Hz} 28 \mathrm{kV}$

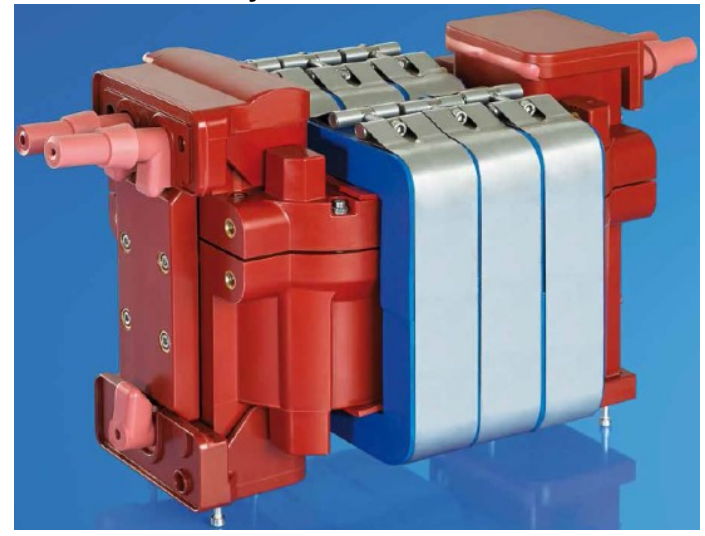

$450 \mathrm{~kW}, 1.8 / 1.8 \mathrm{kV}, 8 \mathrm{kHz}$

STS Transformers, 2015 [7]

Claimed HV insulation to ground 15 kV @ 16 2/3 Hz $P D$ free $A C 50 \mathrm{~Hz} 37 \mathrm{kV}$

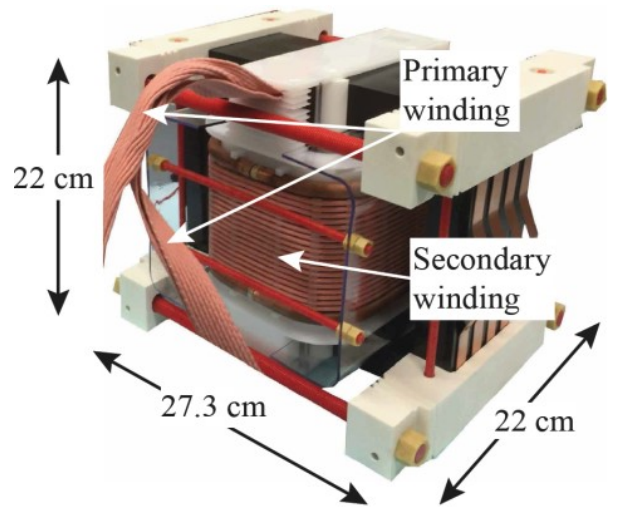

$0.4 / 14.4 \mathrm{kV}, 10 \mathrm{kHz}$

Jaritz et al. (ETH), 2017 [9]

Claimed HV insulation to ground $115 \mathrm{kV}$ DC

$P D$ free $A C 50 \mathrm{~Hz}$ in oil $110 \mathrm{kV}$

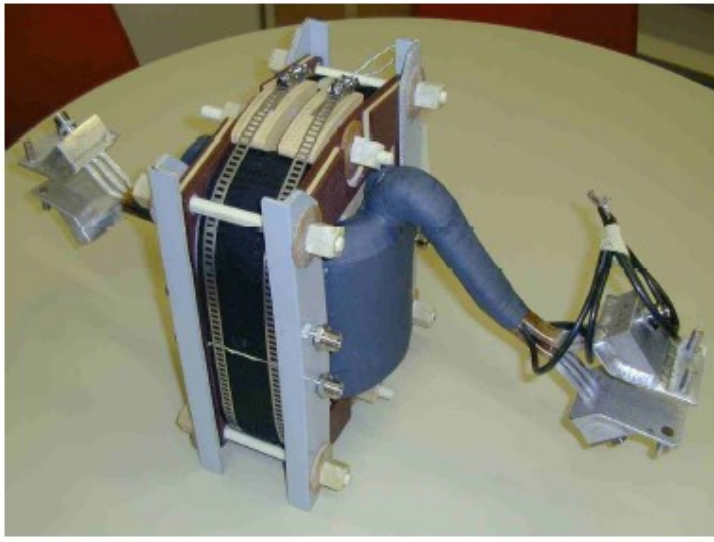

$350 \mathrm{~kW}, 1 / 1 \mathrm{kV}, 8 \mathrm{kHz}$

Steiner et al. (Bombardier), 2007 [6]

Claimed HV insulation to ground 15 kV @16 2/3 Hz $P D$ free $A C 50 \mathrm{~Hz} 33 \mathrm{kV}$

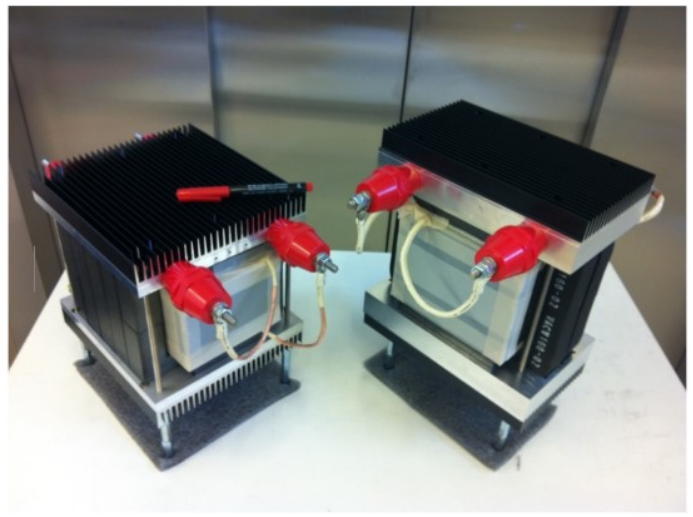

$50 \mathrm{~kW}, 1 / 3 \mathrm{kV}, 5 \mathrm{kHz}$

Bahmani, Kharezy (Chalmers), 2014 [4] [8]

Claimed HV insulation to ground 3 kV DC No information about PD test

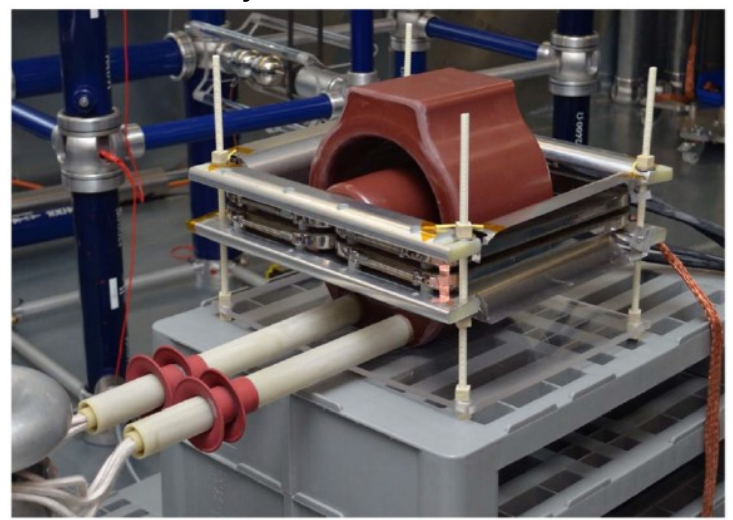

$240 \mathrm{kVA}, 0.6 / 0.9 \mathrm{kV}, 10 \mathrm{kHz}$

Gradinger et al. (ABB), 2017 [10]

Claimed HV insulation to ground $50 \mathrm{kV} \mathrm{DC}$

$P D$ free $A C 50 \mathrm{~Hz} 35 \mathrm{kV}$

Figure 1-2. Examples of MFTs having high HV to ground withstand strength 
Jaritz et al. (ETH) in their paper, published in 2017, have explained the design of an MFT having a high voltage isolation of $115 \mathrm{kV}$ [9]. The transformer is $0.4 / 14.4 \mathrm{kV}, 10 \mathrm{kHz}$. The unit is designed to be used in a system of 8 series $14.4 \mathrm{kV}$ converters (totally $115 \mathrm{kV} \mathrm{DC}$ to ground). The transformer has passed a $110 \mathrm{kV}$ r.m.s. (136\% of $115 \mathrm{kV} / \mathrm{V} 2) 5$ minutes AC PD test inside a synthetic Ester oil. However, all the insulation design is for an $A C$ stress and only based on a $\mathrm{kV} / \mathrm{mm}$ high voltage strength and primitivities values of the material used. No information about the long-term effects of oil to plastic materials used as coil-formers and barriers are presented. Furthermore, no study has been done regarding behaviour of the transformer under HVDC stress.

Gradinger et al. (ABB) in their work published in 2017 [10] have presented a design for a core type cast resin winding, $240 \mathrm{kVA}, 0.6 / 0.9 \mathrm{kV}, 10 \mathrm{kHz}$ prototype transformer. A $150 \mathrm{kV}$ Lightning Impulse (LI) test is also performed. This transformer is claimed to withstand $50 \mathrm{kV}$ HVDC voltage to ground but this has not been considered in the design process nor a DC test has been performed on the manufactured prototype. Even at $A C 50 \mathrm{~Hz}$, the transformers PD free insulation level has been found to be only $35 \mathrm{kV}$.

Only a few have studied a proper winding configuration considering high voltage stresses. Huang et al. have compared foil type winding with Litz windings and explained how an interleaved winding structure can lower the maximum electric field intensity and what is the limitations to do this in their work published in 2017 [11]. Jaritz et al. (ETH) in their paper, published in 2017 [9], have explained how different winding configurations can help with achieving minimum insulation stress. However, none of these have considered a lower breakdown and higher aging under a high frequency square wave regime.

According to Ortiz in his PhD thesis published in 2014 [12] there is a research gap in isolation strategies implemented in designing. There are other challenges regarding the insulation design of an MFT and some of them are mentioned in a tutorial given by Dujic in [13].

There are only a few works that have claimed a high insulation voltage to ground at their transformers' HV side. Insulation designs is based on a $\mathrm{kV} / \mathrm{mm}$ high voltage strength values of the insulation materials. In most of the cases a safety factor of 2 is used. None of the papers have presented an HVDC voltage to ground design.

Jaritz has presented a list of possible solid insulation materials that can be used as barriers in different places in the transformer [9]. However, the comparison is limited to the AC situation based on permittivity of the materials as the voltage distribution at DC steady state is based on conductivities. Information on chemical interaction of the materials with the insulation fluid and on the thermal properties of the materials at high transformer temperature is missing.

\subsubsection{High voltage insulation under HVDC stress}

Gäfvert et al. used an ion drift diffusion model to investigate a composite oil/paper insulation in the converter transformers and compared it with the conventional Maxwell-Wagner model. They also used the optical Kerr effect measurement technic to verify the accuracy of their model. Comparing the measured electric fields inside a two-electrode test object with the ones obtained by simulations, they have deduced that the ion drift diffusion model results in a better estimation of the electric field compared with the Maxell-Wagner model [14]. In the ion drift diffusion model, they have noticed that there is a polarity effect in electric field distribution. They found that their simulation results have a relatively tight coincidence with the measurement [15]. 
Different parameters are used in this complex model which are the positive and negative ions mobilities, their diffusion constants, and also particles dissociation and recombination constants. It seems that the accuracy when measuring such parameters as well as its complexity are the bottleneck of this model [14], [16]. Some non-linear dependency of the material properties is ignored to simplify the used model. The simulation results have coherence with the measurements, but several deviations are observed. After performing some experiments on oil/paper insulation structures that resemble real transformers, finally [14] concludes that in steady state the traditional MW model delivers reasonable results and can be used for transformer dielectric design, although it cannot represent the physical phenomena take place inside the dielectrics.

[17] used Kerr electro-optic technique to study electric field in oil/paper dielectrics. They have applied high DC voltage to two bare electrodes inside various oil insulations and observed that the electric field distribution is uniform for fresh oils and non-uniform for aged oil; having a maximum value close to the electrodes. Also, they have observed a time and polarity dependent stress in oil/paper composite dielectrics. Although these facts show the non-linear behaviour of the dielectrics, they have used a linear Maxwell-Wagner (MW) model which is based on the material's dielectric constants and conductivities. In a linear MW model, the voltage distribution at the instant of the DC voltage application is capacitive, which gradually changes to resistive distribution. The rate of change in electric fields depends on the time constants of the dielectric materials. Although this model cannot represent the non-linear behaviour of dielectrics, but it was shown in [18], [17], [19] that it has an acceptable degree of accuracy. This level of the accuracy has caused the MW model to be the prevalent method in the HVDC dielectric design of transformers [20] , [19], [21] and [22].

A summary of some important activates regarding oil-Barrier insulation system under HVDC stress is presented in Table 1-1.

Table 1-1. A summary of research activities regarding oil-Barrier insulation system under HVDC stress

\begin{tabular}{|c|c|c|c|}
\hline Authors & Theoretical Method & $\begin{array}{l}\text { Validation } \\
\text { Method }\end{array}$ & Modelling \\
\hline $\begin{array}{l}\text { U. Gäfvert, } \\
\text { O. Hjortstam } \\
\text { Y.V. Serdyuk }\end{array}$ & Ion drift Method (Onsager theory) & $\begin{array}{l}\text { Kerr effect measurement, } \\
\text { Maxwell-Wagner simulation }\end{array}$ & $\begin{array}{l}\text { Transformer } \\
\text { Oil-barriers system }\end{array}$ \\
\hline $\begin{array}{l}\text { A. Küchler, } \\
\text { M. Libschner } \\
\text { F. Schober }\end{array}$ & Equivalent RC circuit & $\begin{array}{l}\text { PDC measurement, } \\
\text { Maxwell-Wagner } \\
\text { simulation }\end{array}$ & $\begin{array}{l}\text { Transformer Bushing } \\
\text { Oil-barriers system }\end{array}$ \\
\hline $\begin{array}{l}\text { H. Okubo, } \\
\text { K. Kato } \\
\text { R. Nakane }\end{array}$ & Maxwell-Wagner & $\begin{array}{l}\text { Kerr effect measurement, } \\
\text { DC Breakdown, } \\
\text { Maxwell-Wagner simulation }\end{array}$ & $\begin{array}{l}\text { Transformer } \\
\text { Oil-barriers system }\end{array}$ \\
\hline $\begin{array}{l}\text { G. Chen, } \\
\text { B. Huang } \\
\text { M. Hao }\end{array}$ & & $\begin{array}{l}\text { PEA space charge, } \\
\text { Maxwell-Wagner simulation }\end{array}$ & Oil-barriers system \\
\hline $\begin{array}{l}\text { K. Backhaus, } \\
\text { T. Gabler } \\
\text { R. Fritsche } \\
\text { H. P. Öftering }\end{array}$ & $\begin{array}{l}\text { Ion drift Method } \\
\text { (Poisson-Nernst-Planck) }\end{array}$ & $\begin{array}{l}\text { DC Breakdown } \\
\text { PDC }\end{array}$ & Oil-barriers system \\
\hline $\begin{array}{l}\text { Y. Ebisawa } \\
\text { S. Yamada }\end{array}$ & Linear Maxwell- Wagner & DC Breakdown & $\begin{array}{l}\text { Transformer } \\
\text { Oil-barriers system }\end{array}$ \\
\hline
\end{tabular}

\subsubsection{Importance of measurement of electrical conductivity}

The physical performance of insulation oils under HVDC stress cannot be explained just by their conductivity features. Ion drift and diffusion, and space charge has a great influence on the voltage distribution in this case and can be of use when analysing the time dependent conductivity values [19], [23]. 
When an HVDC voltage is applied to the MFT's HV winding the voltage stress over the insulation layers it is not simply calculatable considering a constant conductivity figures for the materials used as insulation layers. Among others, parameters like Temperature of oil and paper, field strength, prestressing of insulation system and duration of application of voltage should be considered [24]. These parameters affect the ion mobility and density and consequently change the resistivity of materials and the time of stabilization of the coordinated insulation system when HVDC is applied.

Additives like inhibitors, pour-point depressants, antioxidants or antistatic agents are added to the mineral or synthetic oils to make them as a reliable medium for a long-time application of power transformers as possible. Dissolution of these additives, or oil and cellulose oxidation products has a considerable effect on the conductivity of insulating oil or oil immersed cellulose [25]. That is why, even for the same type of oil, there is a vast spreading in the conductivity values depending on difference in producers, production facilities, markings or even periods of production.

Wahlström has presented an equation for dependency of transformer oil and paper board resistivity to the temperature [26] and Beletsky has presented an expression for dependency of transformer oil to electric field intensity[27]. However, one cannot rely completely to these expressions and as mentioned earlier, there are several parameters that can affect conductivity values at the same time.

To sum up, for a successful insulation design, a set of measurements on oil and OIP electrical conductivity values are required.

\subsubsection{High voltage insulation under high frequency stress}

Not only an MFT, but also $50 \mathrm{~Hz}$ power transformers designed to be used as step-up transformers in wind farms are subjected to high frequency transients and high voltage stresses which is a result of resonance. At the moment, to design an MFT, one can rely on insulation materials datasheets for 50 $60 \mathrm{~Hz}$ frequencies and use a conservative safety factor, however in this way the final design can be far from optimized, and also the failure rate will be unknown and finally the designs will not be comparable with each other. Heinemann who has designed one of the high voltage prototypes of MFTs explains the importance of paying attention to effect of the voltage wave form [5].

Nowadays, wind farm transformers are designed and manufactured according to IEC 60076-16. In his $\mathrm{PhD}$ thesis [28], Khanali concentrates on the effect of rate of rise and repetition frequency of high voltage impulses on insulation of transformers. Although the study is on $50 \mathrm{~Hz}$ transformers, the same challenges exists for insulation design of MFTs which operate under square wave voltages and in the $\mathrm{kHz}$ range.

IEC 60851-5, IEC 60243-1 or ASTM D149 specifies tests of electrical properties of insulations at frequencies lower than $60 \mathrm{~Hz}$. IEC 60250 or ASTM D150 are guidelines for the determination of dielectric properties of insulating materials, however no information is given about withstand testing methods of insulation materials at frequencies higher than $60 \mathrm{~Hz}$.

For power transformers, IEC 60076-11 and IEC 60076-16 together with IEC 60076-3 cover dielectric tests only at power frequencies and IEC 60664 and IEC 61558 series are about safety measures for transformers only up to $25 \mathrm{kVA}$. In the rotating machine field there is valuable information, IEC 60034-18-41 describes qualification testing of rotating machines fed from voltage converters under high frequency stress. However, neither standardization nor defined test methods specifically addressed the insulation materials for an MFT which typically runs on medium frequency $(<20 \mathrm{kHz})$ 
square wave shape with rise times in the microsecond range. A series of insulation tests should be performed using a reportative impulse voltage and the results be compared with the data presented in high voltage materials' specification sheets.

Koltunowicz demonstrates the influence of frequency and rise time on the Breakdown Voltage (BDV) and Partial Discharge Inception Voltage (PDIV) of grid transformers [29]. A summary of results published by him [30] clearly shows how an increase in repetition frequency and/or a decrease in rise time can negatively affect the breakdown voltage. According to him, the breakdown occurs after a certain number of impulses. Khanali shows that at medium frequency, although a higher rate of rise causes a higher PD inception voltage, in the case of incepted PD the discharge energy is higher for shorter rate of rise [31].

Kiiza has described how a long PD activity combined with high voltage impulses can degrade an oilpaper insulation system [32]. He has used $\mathrm{AC} 50 \mathrm{~Hz}$ voltage and High Voltage (HV) impulses to demonstrate how this combination, resulting in insulation destruction. Morshuis has published his findings on degradation of solid dielectrics resulted from an internal PD [33]. In case of an MFT, instead of sinusoidal $50 \mathrm{~Hz}$, a medium frequency square wave can be used to create the partial discharge.

To sum up, very limited activities have focused on insulation design requirements of high voltage MFTs operating under a medium frequency square wave regime. The missing gap extends from an insulation material selection stage to reliable long-time operation. For example, the material data sheets do not present the breakdown strength under medium frequency square wave stress.

\subsubsection{Importance of determining leakage inductance of MFTs}

To achieve the lowest loss by the Zero-Voltage Switching of a Dual Active Bridge converter, it is crucial to precisely calculate the embedded Leakage Inductance of the used Medium Frequency Transformer (MFT). An effective method shall be proposed for calculation of the leakage inductance of the MFT.

A DAB can benefit from the MFT's leakage inductance $\left(L_{s}\right)$ to transfer the power between the input and output bridges and this will result in high power density unit. By controlling the switching on the two sides, the square wave voltage from the primary and the secondary converters will be adjusted to have a defined phase shift relative to each other. Such a transformer serves as an inductance, in addition to its natural duty of voltage adoption between the primary and secondary bridges (see Figure 2-2b). In other words, the design of the transformer should fulfil the criteria of having a determined leakage inductance in addition to keeping the specified isolation, efficiency and the thermal requirements [34].

The core loss is highly dependent on the duty cycle and the rise time of the wave shapes [35]. The lowest loss is achieved when zero-voltage switching (ZVS) is achieved, which among other factors depends on the phase shift, $\varphi_{\min }$ between the primary and secondary bridges. It would thus be of great importance to accurately evaluate and implement the leakage inductance of such a transformer in the design stage.

By using accurate analytical methods, time-consuming FEM simulations can be avoided in the design stage. Several methods for the calculation of the leakage inductance of a transformer can be found in the literature [36-40]. In the classical method for calculation of leakage inductance the effect of the 
core is neglected, and it is also difficult to consider asymmetries in distributions of ampere-turns. A popular approach to calculate axial and radial components of the leakage field taking the effect of the iron core into account is the method of images. In this method the effect of the iron core is represented by infinite images of the coils in all directions inside the core window [36]. A method based on double Fourier series was extended in [39] to calculate the leakage reactance for an irregular arrangement of windings. The ampere-turn density distribution as well as the flux distribution contains components which vary harmonically along both the $\mathrm{x}$ and $\mathrm{y}$ axes.

The loss characteristics of Litz wire windings is completely modelled and demonstrated in [41] and [42]. In recent works including Bahmani [34], Ouyang [43], and Zhang [44], the effect of frequency on the leakage inductance of MFTs has been studied and the accuracy of the presented methods have been confirmed by experimental measurements up to $2 \mathrm{MHz}$. In most of previous works, the Dowell method [45] or its improved form [46, 47] has been utilized. The Dowell method is based on the calculation of losses in long foils under axial leakage flux using a one-dimensional field solution. In [48] the equivalent area method has been used for a modification of Dowell's method to calculate the losses of windings with round (or Litz) wire. In [44] the skin and proximity effect losses of a round conductor subjected to a uniform applied field are used for obtaining a frequency-dependent leakage inductance relation of a high frequency transformer with Litz wire windings.

In all of these studies, it is assumed that the leakage flux inside the windings and in gaps between windings is completely axial, while the effect of the radial component of the leakage flux is considered separately. This is accomplished by applying the Rogowski factor to the average height of the windings to obtain a higher equivalent winding's height [49]. The error of using the Rogowski factor increases, especially when the difference in heights of the windings is significant or when the ratio of height to width of the windings is less than a certain level. Another important aspect is the inaccuracy of the classical leakage inductance formulas (which can be found in [36]) for MFTs with axisymmetric geometry. The shape of the leakage flux in a planar geometry is significantly different from the one in axisymmetric geometry, especially for fairly short windings with unequal heights. Ignoring this fact also can cause errors in the leakage inductance value.

\subsubsection{Importance of determining transformer losses}

The information about the losses which produces heat inside the transformer is valuable for designing an HVDC MFT in two ways. First since MFTs have high power densities [50] and heat dissipation is extra important and second, because the DC insulation design has a direct dependence to the electrical conductivity values of the insulation materials which are themselves, temperature dependent parameters [51,52]. In other words, energising and loading the transformer results in temperature changes in core and coils and consequently distresses the multilayer insulation system of the transformer. In addition, to achieve the highest efficiency of a DAB converter, it is crucial to accurately calculate the winding losses of the used MFT.

The main origins of the losses in a transformer are the losses in the cores when an alternatively changing flux having a certain density is induced in it and losses is in the windings when the high frequency current passes through them. These losses produce heat which warms up the environment which the active part is placed in (in this project transformer oil). The amount of heating will be varying during the transformer operation depending on the excitation voltage level and loading current level. Normally the losses are calculated and measured for the worst case when the transformer is supplied at its rated voltage and the rated current. 
Winding losses are caused by ohmic loss in the current carrying windings and additional losses because of proximity and skin effect and core losses consist of static hysteresis losses, classic eddy current losses and excess eddy current losses [36]. Additional losses are because of induced capacitive/inductive currents in the adjacent construction conductive parts.

In addition, the losses will contribute to the warming up of the transformer oil which will affect the conductivity and consequently the voltage stress distribution under an HVDC high voltage application [52]. The amount of produced heat is used to design the oil circulation and cooling system of the transformer.

At high frequencies, the losses in the copper MFT winding conductors will drastically rise because of the skin effect in the conductors, and the proximity effect to the adjacent conductors. The winding losses are calculated using linear superposition of the losses caused by different harmonic contents of the applied AC current [53]. The voltage and current waveforms can be observed in Figure 2-1a.

Having access to a set of precise formulas and methods, for the calculation of winding losses with an acceptable accuracy, is of high importance. Wide frequency range, possibility of having a free selection of number of layers, and number of turns per layer are among the features that should be considered during the development of new methods. Previous works [42,54-56] propose formulas to calculate the $A C$ resistance of the conductors under high frequency switching conditions. These proposed analytical methods for calculation of winding losses are based on empirical relations originally developed for foil windings, which have been modified by researchers to be applicable for Litz wires. However, these methods are approximate, and their application is time consuming, and requiring many simplifying assumptions to solve the problem.

Dielectric losses cause heating of the insulation material which is dependent to the frequency and magnitude of the applied voltage, and to the capacitance and loss angle of the insulation material as explained [36] is negligible at $5 \mathrm{kHz}$ compared with the core and winding losses [3] and is not included in the scope of this work.

\subsection{Required information on oil type MFT's components}

Inside the power transformers, solid insulation materials are used for several purposes like isolation of conductors, as coil-formers, as insulation barriers in the liquid insulations or as mechanical isolators. Gas (SF6, air, etc.) or liquid (mineral, ester, silicone oils, etc.) are also used in combination with the solid insulations for this purpose.

The liquid insulation is used for different purposes in a transformer. The most important functions of transformer oil are isolation (alone and as a saturating material for cellulosic barriers), cooling, absorbing the humidity of solid insulations, an arc-quencher and a carrier of information about the active-part's health [57]. In the case of the prototype and the real scale transformer, two oil types are considered, the mineral oil which has the most well-known characteristics for AC and HVDC applications and is used for large converter transformers [58] and synthetic ester oil which is biodegradable [59] [60]. Because of nonlinear electrical behaviour, a high volume of the research is concentrated to characterisations of these types of oil for HVDC applications [61] [62] [52].

A comprehensive review about environmental impacts of using natural ester is presented by Asano [59]. This type of oil is an environment-friendly replacement for mineral oil which has widely been in 
use in power transformers. An ISO/IEC committee 10: Fluids for electro-technical applications are preparing a guideline for the use of natural ester oils in electrical equipment.

Density, Kinematic viscosity, Flashpoint, Pour point, Interfacial tension, Corrosive sulfur content, Water content, Acidity, Breakdown voltage, Impulse breakdown voltage, Gassing tendency and Oxidation stability are among those important parameters which should be characterized for an insulation oil before deciding if it is a suitable replacement for mineral transformer oil [57].

Solid barriers act as insulation when AC or DC voltage is applied to an oil type transformer. In addition, barriers act as separators to reinforce the electrical strength of long oil gaps [63]. Both volume and surface (creepage) dielectric strength of the solid insulation are important. In this section we have concentrated on withstand of the insulation under HVDC stress. The conductivity of solid insulation which is a non-linear function of applied voltage, temperature and the time of application of voltage [52] is studied. Solid insulations are polarised and contribute to concentration of space charges in its interface with the insulation oil which shall be considered for a successful HVDC insulation system design.

Two types of solid insulation materials are used for manufacturing power transformers; cellulosic and Nomex materials [58]. Cellulosic transformer board is made of unbleached sulfate cellulose from slow and dense growing tree woods [63]. Nomex transformer board is made of synthetic crystalline aramide. The most important advantages of Nomex is its thermal stability, and it is very high resistance to aging. Its disadvantage is its tendency to attract the particles if it is statically charged [24].

As the prototype transformer is planned to be of oil type, in this project we will limit the scope of the materials to solid cellulosic materials (as barriers) in oil, plastics in air (as bushings) and finally mineral and ester transformer oils.

\subsection{Purpose \& contributions}

The purpose of this work is to develop a medium frequency transformer to be used in a special $D C / D C$ converter for series connection of the outputs of wind turbines. The uniqueness of the concept is that the transformer apart from transmitting energy from the wind turbine side to the collecting radial side has to handle a high DC voltage as well. Especially for offshore applications this system has great cost advantages.

The following contributions has been made

- Concept proof of an oil type power transformer with a high voltage DC design concept, through both a theoretical design as well as a prototype that has been built

- Characterisation of an eco-friendly biodegradable transformer oil for HVDC transformer applications,

- Introduction of reliable high frequency characterisation test methods to medium frequency transformer designers,

- Development of a method, based on Non-Linear Maxwell Wagner (NLMW) relations, to design an HVDC MFT transformer.

- A demonstration of how the leakage inductance constraint shall be realised during the insulation design in an appropriate way. 
In this work, the DC series concept further developed one step closer to pre-commercialization, i.e. from TRL 1 to about 2.

\subsection{List of publications}

\subsubsection{Journal articles}

I. M. Kharezy, H. R. Mirzaei, Y. Serdyuk, T. Thiringer and M. Eslamian, "A Novel Oil-immersed Medium Frequency Transformer for Offshore HVDC Wind Farms," Submitted to IEEE Transactions on Power Delivery, June 2020, Under review

II. M. Eslamian, M. Kharezy, and T. Thiringer, "An accurate analytical method for leakage inductance calculation of high-power shell type medium frequency transformer with circular windings," Submitted to IEEE Transactions on Power Electronics, July 2020, Under review

III. B. Alikhanzadeh, T. Thiringer and M. Kharezy, "Requirement on Leakage Inductance in Singleand Multi-level DAB Converters: Algebraic Formulations and Sensitivity Analysis," Submitted to IEEE Transactions on Power Electronics, May 2020, Under review

\subsubsection{Conference articles}

I. M. Kharezy, H. R. Mirzaei, T. Thiringer 2, J. Rastamo, M. Svensson, T. Nieminen and Y. Serdyuk, "Performance of Insulation of DC/DC Converter Transformer for Offshore Wind Power Applications," Conference on Electrical Insulation and Dielectric Phenomena, CEIDP2020, NJ, USA, October 18-22, 2020

II. M. Kharezy, M. Eslamian, and T. Thiringer, "Estimation of the winding losses of Medium Frequency Transformers with Litz wires using an equivalent permeability and conductivity method," 22stEuropean Conference on Power Electronics and Applications, EPE2020, Lyon, France, September 7-11, 2020

III. M. Kharezy, M. Eslamian and T. Thiringer, "Insulation design of a medium frequency power transformer for a cost-effective series high voltage DC collection network of an offshore wind farm," $21^{\text {st }}$ International Symposium on High Voltage Engineering, ISH2019, Budapest, Hungary, August 26-30, 2019

IV. M. Eslamian, M. Kharezy and T. Thiringer, "Calculation of the leakage inductance of medium frequency transformers having rectangular-shaped windings using an accurate analytical method," $21^{\text {st }}$ European Conference on Power Electronics and Applications, EPE2019, Genova, Italy, September 2-6, 2019

V. B. Alikhanzadeh, T. Thiringer and M. Kharezy, "Optimum Leakage Inductance Determination for a Q2L-Operating MMC-DAB with Different Transformer Winding Configurations," 20th International Symposium on Power Electronics, Ee2019, Novi Sad, Serbia, October 23 - 26, 2019

VI. M. Kharezy and T. Thiringer, "Challenges with the design of cost-effective series DC collection network for offshore wind-farms," 17th Wind Integration Workshop on Large scale Integration of Wind Power into Power Systems as well as on Transmission Networks for Offshore Wind Power Plants, WIW2018, October 17-19, Stockholm, Sweden, 2018 


\section{Theory}

\subsection{HVDC MFT for series DC integration layout}

The Dual active bridge (DAB) topology is easy to control, has wide zero voltage switching (ZVS) region without a need for snubber circuits in contrast to single active bridge converters (SAB) which have the disadvantage of hard switching on the output diodes. Moreover, it can operate at a higher frequency and power level compared to full bridge converters (FBC) and has no need for high voltage and current resonant capacitors. A final advantage is that is has no need for a large resonant inductor as compared with series resonant converters (SRC). The leakage inductance of the MFT is used as a resonant inductor inside a $D A B$ and this, results in higher efficiency and lower volume and price. Although a dual $\mathrm{H}$ bridge (DHB) has a lower number of parts, the circulating reactive current in the MFT is lower in the case of DAB [3]. As explained, the Dual-Active Bridge (DAB) is one of the proper converter topologies to be used as part of an isolated DC/DC converter for sea-based wind-farm application.

An MFT is in a DAB set-up operated using square wave voltage stress as connected between two $\mathrm{H}$ bridges in the DAB DC/DC converter (see Figure 2-1).

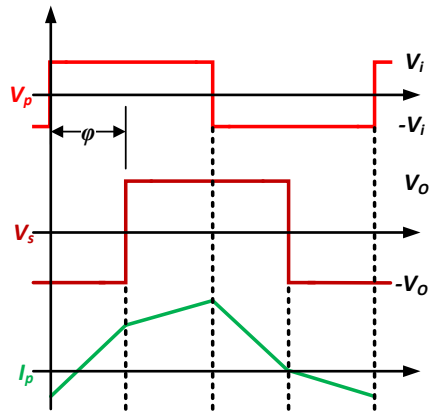

(a)

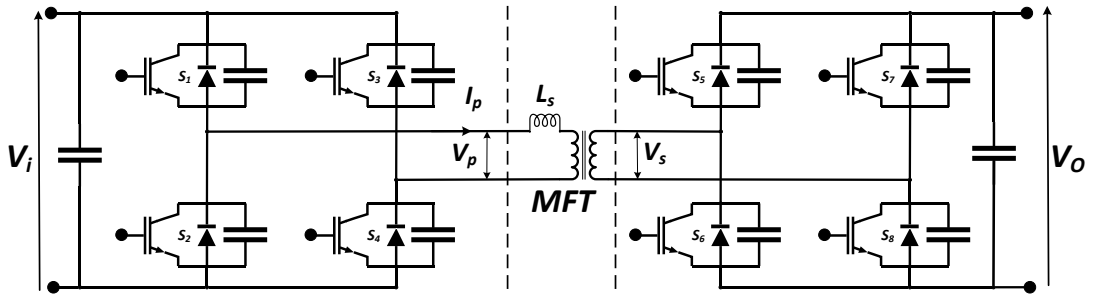

(b)

Figure 2-1. An MFT having an embedded inductance inside a DAB; Voltage \& current wave-shapes (a), An MFT between two stages of a DAB acting as a series inductance (b)

In the series DC concept, DC voltage supplied by series connected converters will be directly transferred to a suitable grid connection point on land where it is feed into the main electrical system (see Figure 2-2).

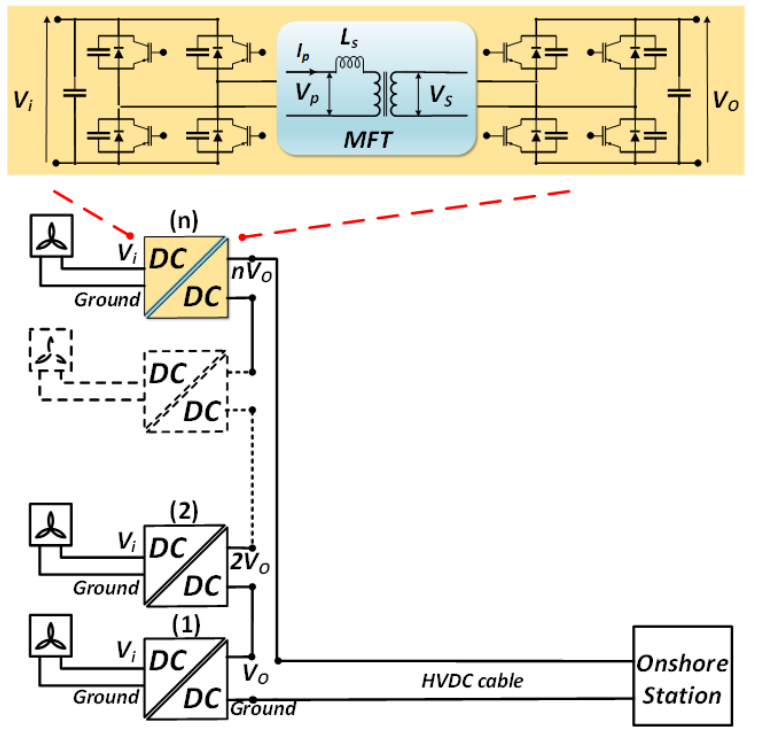

Figure 2-2. MFT inside a DAB as part of a series connected bridges 
The DC/DC converter which have the highest place (highest potential to ground) in the series connected wind farm concept (number $n$ ) can experience a high DC voltage $\left(n V_{o}\right)$ superimposed with a medium frequency square voltage of the secondary $\left(V_{s}\right)$ of a few kilovolts to ground while the primary side have the symmetrical voltage of $V_{p}$ to Ground (see Figure 2-3).

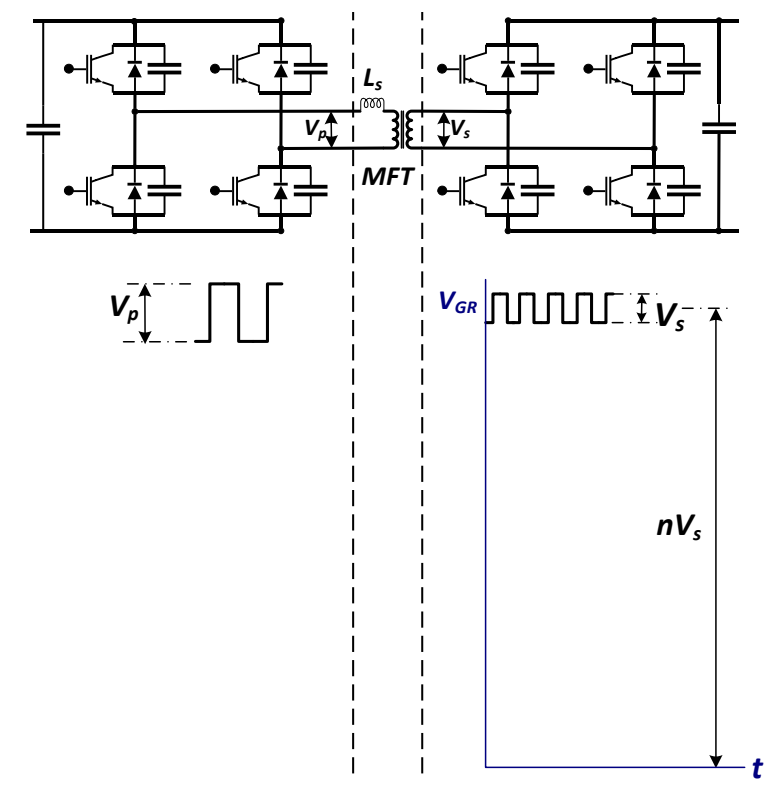

Figure 2-3.Voltage to Ground (VGR) at Secondary side of the MFT

This means that the transformer secondary winding - core, secondary winding - primary winding and secondary winding - body insulations should be dimensioned for a High DC Voltage (HVDC insulation concept).

At the same time, the primary winding - core, primary winding - body and turn-turn insulations both for $\mathrm{LV}$ and $\mathrm{HV}$ windings should be dimensioned for a medium frequency square wave voltage.

As an example, in the ideal case, if 30,1:3 kV DC/DC converter be coupled in series, the voltage at the secondary terminals of the transformer number 30 will be $87 \mathrm{kV}<V_{G R}<90 \mathrm{kV}$. If each turbine delivers 5 $\mathrm{MW}$, the total deliverable power will be $150 \mathrm{MW}$ at the voltage of $90 \mathrm{kV}$. In practice there needs to be a certain over installation of turbines to handle the varying power of the turbines as well has to handle the situation when some turbines are out of operation. In contrast to a normal shunt connected set of turbines that feeds out current to a common DC voltage, here the current between the two DC-voltage buses is the same for all turbines in a series connection.

In order to realise this system, the key issue is to have a transformer within the wind turbine DC/DC converter that apart from transmitting the power from the wind turbine to the collection radial, also is able to handle a very high common mode DC voltage to ground.

\subsection{HVDC MFT's design challenges and methods}

The key challenges with the design of a high voltage are introduced in [50]. Here the issues related to this work is highlighted.

\subsubsection{Non-suitability of dry insulations for high voltage DC transformers}

Insulation materials operating under HVDC conditions will absorb more contaminations compared to the case of AC operation. Under AC operation the net absorption effect is near zero. In addition, the electrical field distribution on the surface of the insulation material is purely resistive and therefore, 
several factors can change the field distribution along the discharge paths; among others, the nonlinear nature of surface resistivity, charge accumulation and surface contamination and humidity can be mentioned here [64]. In addition, as Figure 2-4 demonstrates, under AC excitation, with the application of few kVs there will be corners with an electric field easily exceeding the corona inception voltage in air.

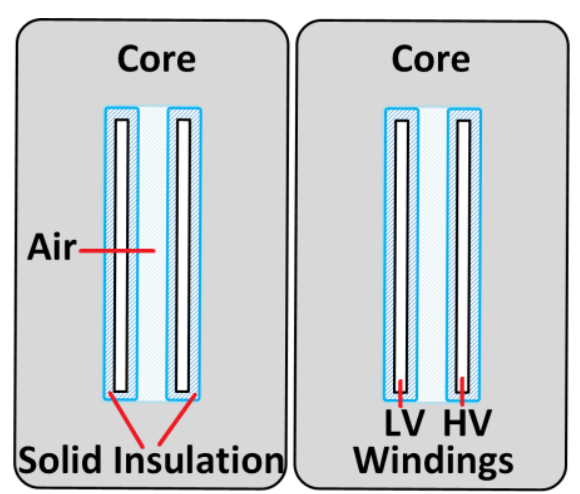

(a)

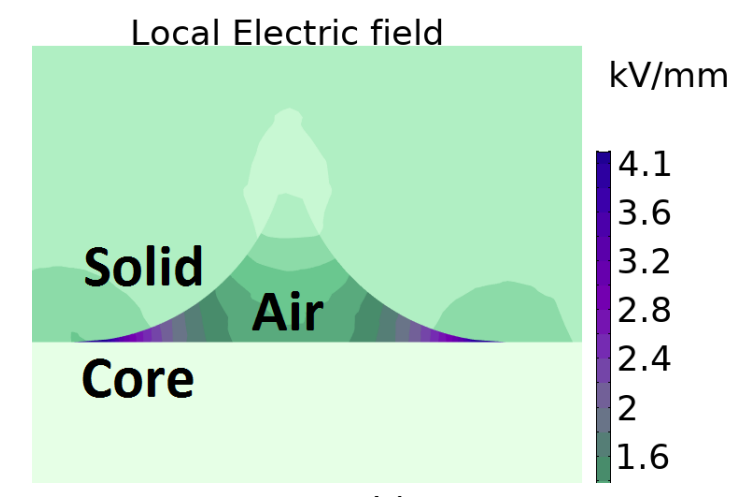

(b)

Figure 2-4. High electric stress in the air in the vicinity of solid insulation of windings; Previously build dry type MFT prototypes [8] (a), Field stress at the corners (b)

\subsubsection{0il type high voltage transformers}

Oil filled high voltage power transformers have been operating successfully for the past century in AC substations. For high power and voltage applications, mineral oil impregnated paper and pressboards have been the dominant technology in use [65]. A thick steel tank protects the active part from external contaminations, a metal core is surrounded by the windings and the oil is used as a reliable component in the insulation system and as a cooling medium (see Figure 2-5).

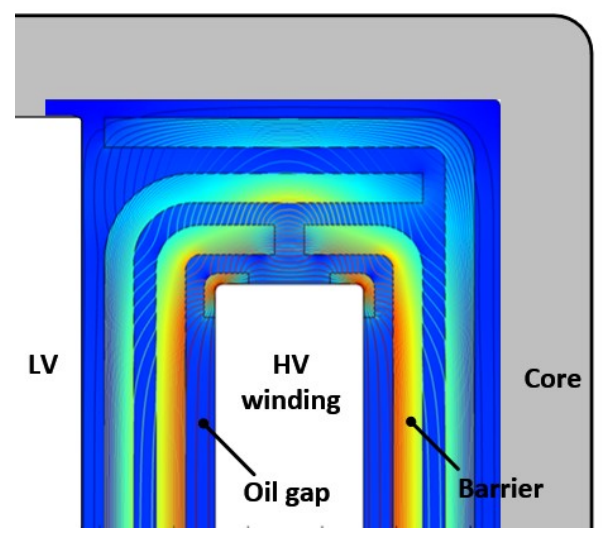

Figure 2-5. The core window filled with the oil, the distance between HV winding is divided to smaller gaps by barriers

For a DC design, the thickness of the solid barriers is important. The position, form, and overlapping of the solid barriers must be skilfully taken into consideration. Local temperature, electric stress, humidity and oil flow inside the insulation system can change the conductivity of the insulation materials and should also be considered for a successful DC design process.

\subsubsection{Transformer insulation under AC stress}

Under AC stress, the voltage distribution is determined by the permittivity of the insulation materials combined as an insulation system. At steady state DC it is the conductivity of the insulation materials which determines the voltage distribution. For an oil paper insulation system, the low permittivity oil which experiences a high voltage potential difference under $A C$ will take less voltage drop then under 
DC due to the lower conductivity compared with solid paper or pressboard insulation. When a DC voltage is applied to the transformer, first it is the permittivity values which determine the voltage distribution and at the steady state condition, the distribution is based on conductivity values [66].

The permittivity values have a weak dependence on the temperature while the conductivity values of both oil and paper, change with operating temperature. In addition, conductivities can change depending on the magnitude and duration of electrical stress applied at a function of time [52].

\subsubsection{Effect of solid barriers at oil type transformers}

Barriers can introduce physical improvements to an insulation system operating under AC regime and it is better to have a finer division in an insulation distance in transformer oil to gain more withstand voltage (see Figure 2-5) [63]. For an oil volume between two electrodes, the insulation strength is influenced by, gap distance, oil volume, electrode surface form and isolation coating of electrodes. The lower the gap distance, the higher the breakdown voltage per millimetre [65]. Therefore, the electric strength of a space between two electrodes in oil increases by inserting insulated barriers. Such a barrier has an influence both on discharge initiation process at the electrode surface and on discharge growing process at the oil gap.

\subsubsection{Voltage distribution under AC stress}

For an oil gap between two conductors divided into several gaps by solid barriers, the uniform electric stress can be calculated by [65]

$$
\frac{V}{d}=\frac{(n+1) \frac{\varepsilon_{\text {Oil }}}{\varepsilon_{B}} d_{B}+n d_{\text {Oil }}}{(n+1) d_{B}+n d_{\text {Oil }}} E_{0}\left[\frac{d_{\text {Oil }}}{1 m m}\right]^{-a}
$$

where $V$ is the applied voltage, $d$ is the total distance between the conductors that shall be minimized, $n$ is the number of oil gaps, $\varepsilon_{O i l}$ and $\varepsilon_{\mathrm{B}}$ are the permittivity of oil and barrier respectively, $d_{O i l}$ and $d_{B}$ are the thickness of the oil gaps and barriers respectively, $E_{O}$ is the PD inception field strength for a $1 \mathrm{~mm}$ gap [63] and $a$ is slope of the straight lines of the PD inception field strength-oil duct width in log-log curves [63].

For an AC Non-uniform field distribution, at the corners of the winding having a highly non-uniform or for long oil gaps like the high voltage lead exits, the local average field stress $E_{a v}(z)$ for each path length can be calculated from the following relation

$$
E_{a v}(z)=\frac{1}{z} \int_{0}^{z} E\left(z^{\prime}\right) d z^{\prime}
$$

For each critical insulation pass, the resulting curve must be compared with the maximum permissible (Partial discharge free) field strength curve $E_{b d}(z)$. This curve is based on a set of empirical measurements [65] and is given as

$$
E_{b d}=E_{0} d^{-0.37}
$$

where $E_{b d}$ is the strength of an oil gap of length of $d \mathrm{~mm} ; E_{0}$ is equal to 17.5 and $21.5 \mathrm{kVr}$.m.s./mm for bare and covered electrodes, respectively;

Finally, a safety factor curve is calculated as

$$
S F(z)=E_{b d}(z) / E_{a v}(z)
$$

which should be higher than 1 along all its length. This process should be repeated for all suspected critical insulation regions in the transformer. 
Figure 2-6 demonstrates a selected critical path, absolute value of the local stress, averaged field stress curves along the paths, the maximum permissible field strength and safety curves under 125 $\mathrm{kV}$ AC for a sample transformer.

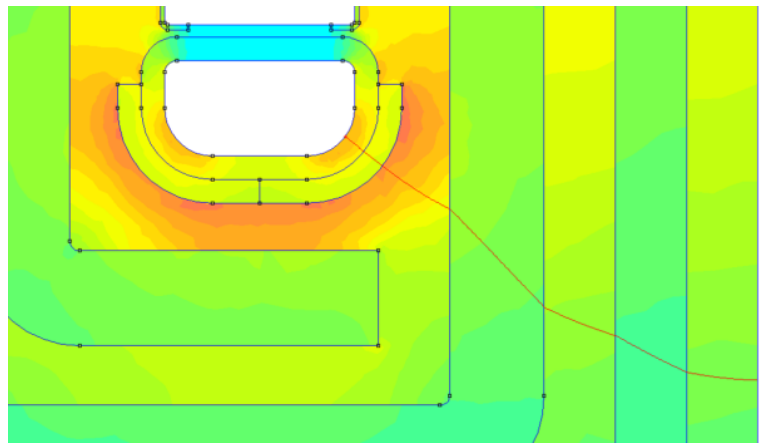

(a)

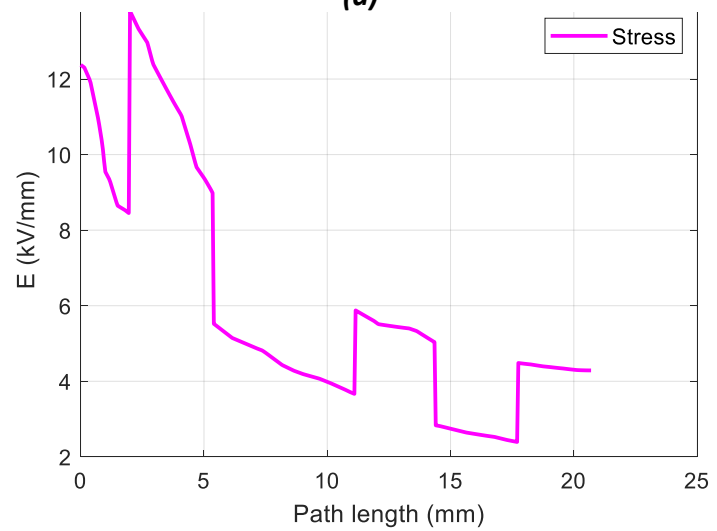

(c)

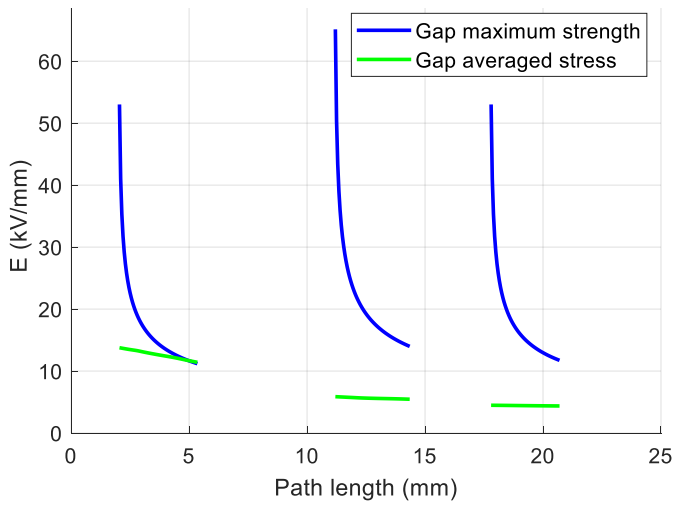

(b)

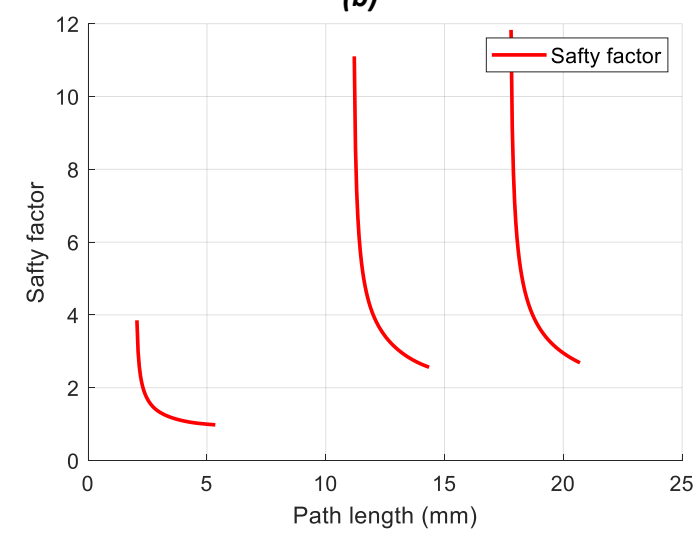

(d)

Figure 2-6. Sample critical path curves for an oil gap; A streamline pass from HV winding shield to the core body (a), Comparison of the maximum and averaged field curves in the respective oil gap lengths (b), Absolute value of the local stress along the field line(c), Safety factor curves per oil gap (d)

\subsubsection{AC design, creepage distances}

The same evaluation method as for the oil gap is applied when the electric strength over the insulations' interface is under consideration. First along the creepage path, the tangential field strength values shall be rearranged to a steadily ascending profile [65]. Then an averaging shall be performed on a path length between two points where the tangential stress is higher than 0.1 $\mathrm{kV} / \mathrm{mm}$ [22]. On the interface of the transformer board with the oil, the maximum permissible creepage strength is $30 \%$ lower than the permissible strength for an oil gap of the same length [65]. Figure 2-7 demonstrates a selected critical path, sorted values of the tangential local stress, averaged field stress curves along the path and the maximum permissible field strength curves under $125 \mathrm{kV}$ AC on a sample transformer. 

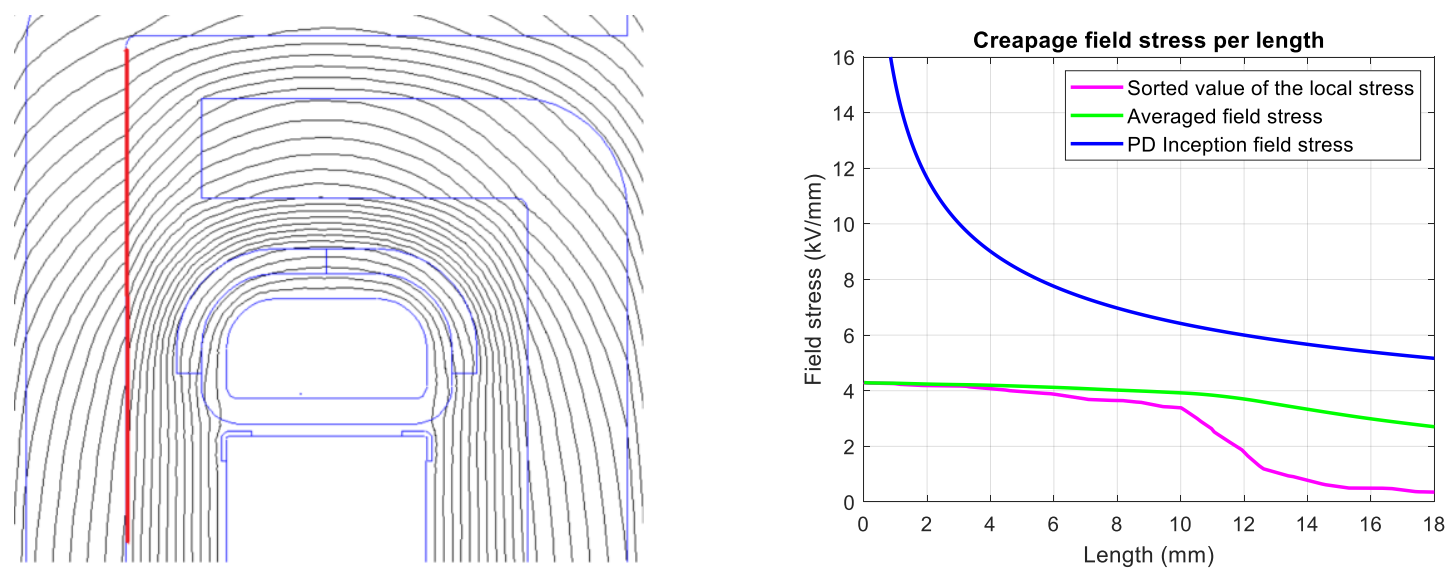

Figure 2-7. Sample creepage path curves on an insulations' interface

\subsubsection{Insulation under DC stress}

To handle a very high DC voltage applied to the high voltage winding, the transformer high voltage winding insulation should withstand induced $A C$ voltage from the low voltage winding and the superimposed DC voltage applied from its HV terminals. Steady state DC and repeated pulsed AC voltage stress is present. A combined HVDC and AC insulation design of the MFT is discussed in [66]. The study shows that for a dry-type MFT this is difficult to accomplish with a relatively high voltage to ground insulation. Oil-type insulation is thus more suitable for an AC design, the long oil gaps shall be divided by a number of solid barriers.

Figure 2-8 demonstrates an electrical circuit equivalent diagram of an insulation system when a DC step voltage is applied on it. The voltage distribution under AC stress is based on permittivity values of the insulation materials, i.e. capacitive properties. At the moment of application of DC voltage, the distribution is similarly based on permittivity values. Later it is a transition time, when the frequency dependent polarisations which have started with the voltage change, ends and finally the voltage distribution is based on the conductivities of insulation materials i.e. resistive properties.

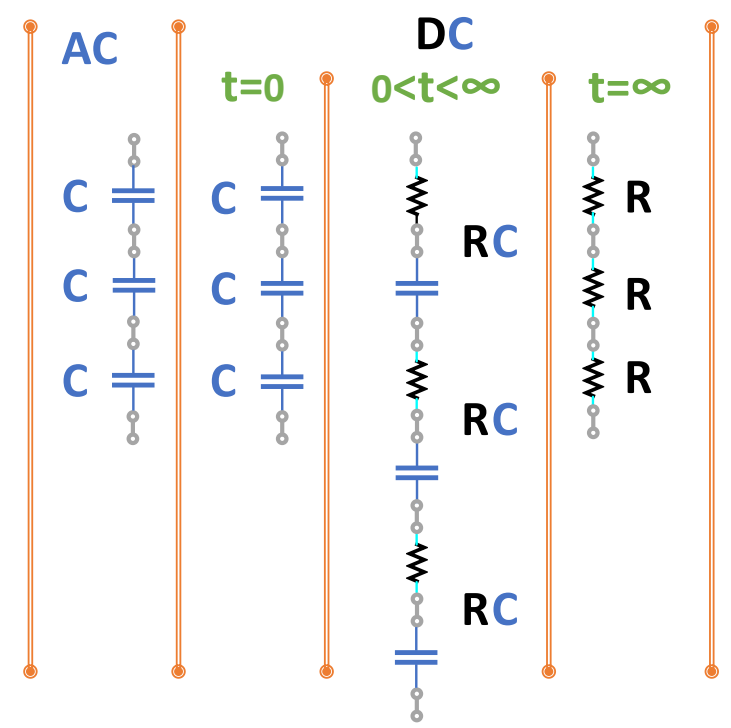

Figure 2-8. Behaviour of an insulation layer stressed with DC step voltage

\subsubsection{Oil-paper insulation system under DC stress}

For AC voltage stress, the electric field is higher in the oil gap having a lower permittivity than in the barriers which have a higher permittivity and it is the oil gap which has a dominant effect on the AC voltage withstand strength. However, for DC voltage stress, the electric field is lower in the oil gap 
having a higher conductivity than in the barriers which have a lower conductivity and it is the barrier thickness which has a dominant effect on the DC voltage strength. As a result, a proper design for an HVDC MFT should include both a high volume of oil and thick solid paper insulation.

Directly after switching in the high HVDC voltage, the capacitive field is charged to a certain HVDC voltage level and as explained, it is the permittivity of the materials which determines the voltage drop over the combined insulation system. After a long time following the applying of HVDC, the resistively divided field is supplied with HVDC voltage and it is the conductivity of the materials which determines the final voltage drop over the combined insulation system. This is demonstrated by the FEM simulations presented Figure 2-9.
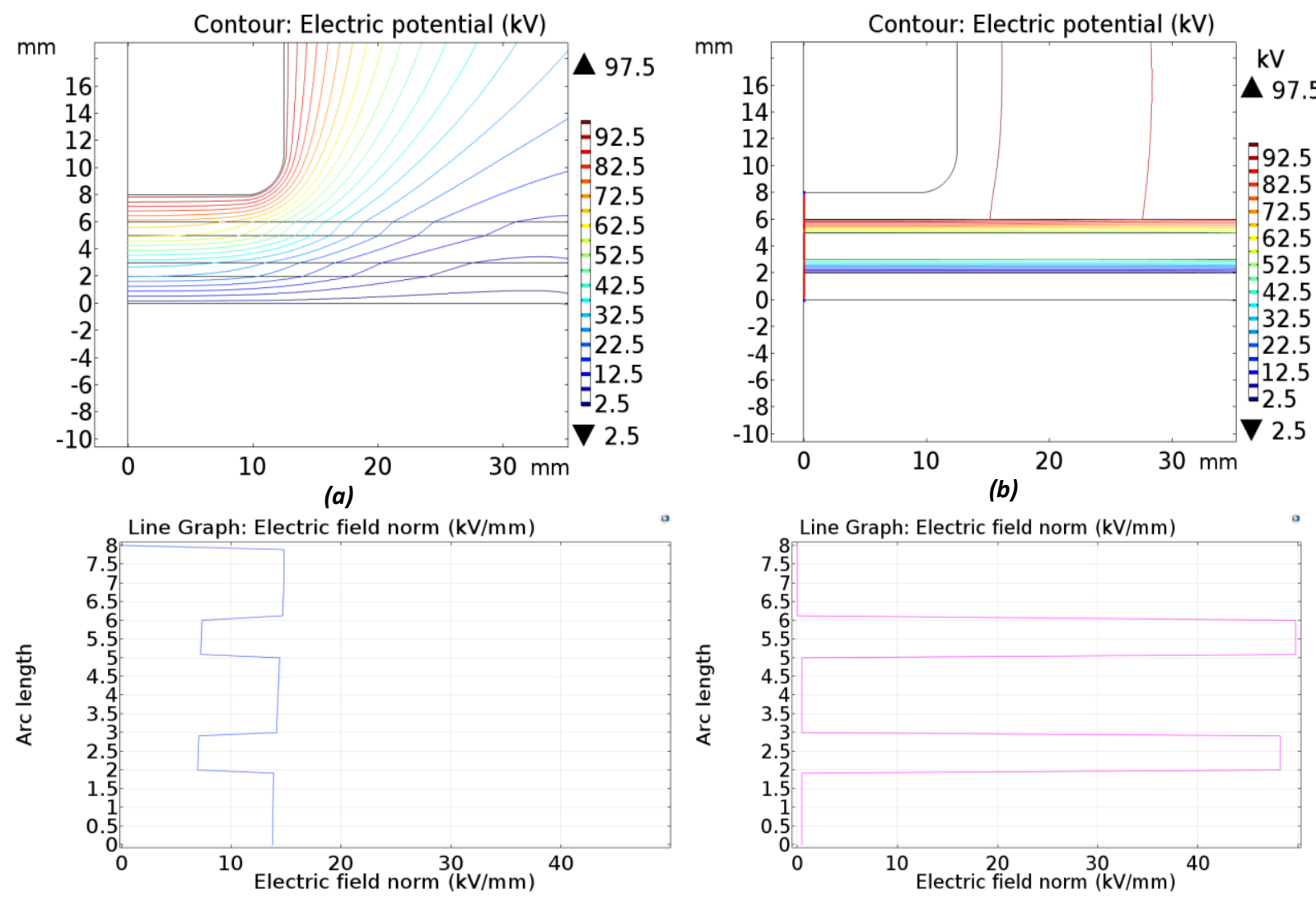

(c)

(d)

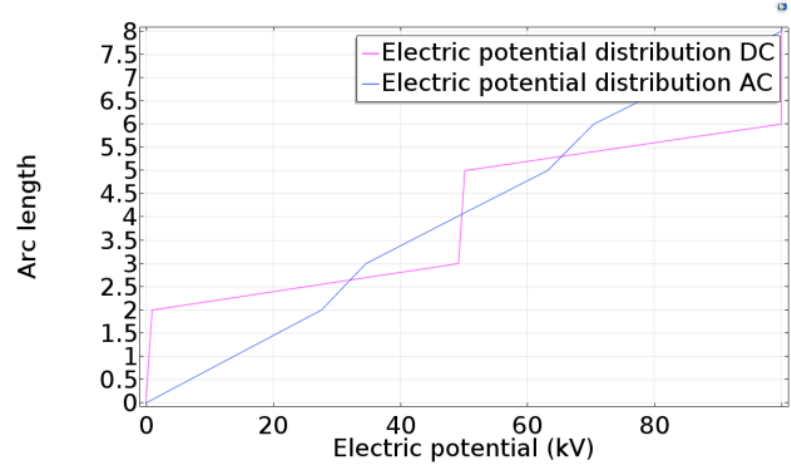

(e)

Figure 2-9. Two electrodes supplied with $100 \mathrm{kV}$ to ground, and two $1 \mathrm{~mm}$ board barriers inside mineral transformer oil, demonstrating the difference between $A C$ (blue) and DC (red) voltage distribution and effect of solid barriers; Equipotential lines distribution under AC supply (a), Equipotential lines distribution under DC supply (b), AC field strength on $8 \mathrm{~mm}$ between two electrodes (c), DC field strength $8 \mathrm{~mm}$ between two electrodes (d), Electric potential distribution (e) 


\subsubsection{Effect of unequal conductivities on varying voltage distribution under DC stress}

Figure 2-10 demonstrates an insulation system consisting of three different insulation layers. On the interface of the two dielectrics, the change of electric charge densities, $D$, with time is equal to the difference on current densities $J$ following the relation

$$
J_{2}-J_{1}=\frac{d}{d t}\left(D_{1}-D_{2}\right)
$$

Therefore, for a three-layer configuration as presented in Figure 2-10 the following differential equation system is valid,

$$
\begin{aligned}
& \sigma_{2} E_{2}-\sigma_{1} E_{1}=\frac{d}{d t}\left(\varepsilon_{1} E_{1}-\varepsilon_{2} E_{2}\right) \\
& \sigma_{3} E_{3}-\sigma_{2} E_{2}=\frac{d}{d t}\left(\varepsilon_{2} E_{2}-\varepsilon_{3} E_{3}\right) \\
& d_{1} E_{1}+d_{2} E_{2}+d_{3} E_{3}=V
\end{aligned}
$$

$E$ is the electric field intensity, $V$ is the applied voltage, $\sigma$ is the conductivity and $\varepsilon$ is the permittivity.

$$
\left[\begin{array}{cc}
\varepsilon_{1} & -\varepsilon_{2} \\
\frac{\varepsilon_{3} d_{1}}{d_{3}} & \varepsilon_{2}+\varepsilon_{3} \frac{d_{2}}{d_{3}}
\end{array}\right]\left[\begin{array}{l}
\frac{d E_{1}}{d t} \\
\frac{d E_{2}}{d t}
\end{array}\right]=\left[\begin{array}{cc}
-\sigma_{1} & \sigma_{2} \\
\frac{-\sigma_{3} d_{1}}{d_{3}} & -\left(\sigma_{3} \frac{d_{2}}{d_{3}}+\sigma_{2}\right)
\end{array}\right]\left[\begin{array}{c}
E_{1} \\
E_{2}
\end{array}\right]+\left[\begin{array}{c}
0 \\
\frac{\sigma_{3}}{d_{3}}
\end{array}\right] V(t)+\left[\begin{array}{c}
0 \\
\frac{\varepsilon_{3}}{d_{3}}
\end{array}\right] \frac{d V(t)}{d t}
$$

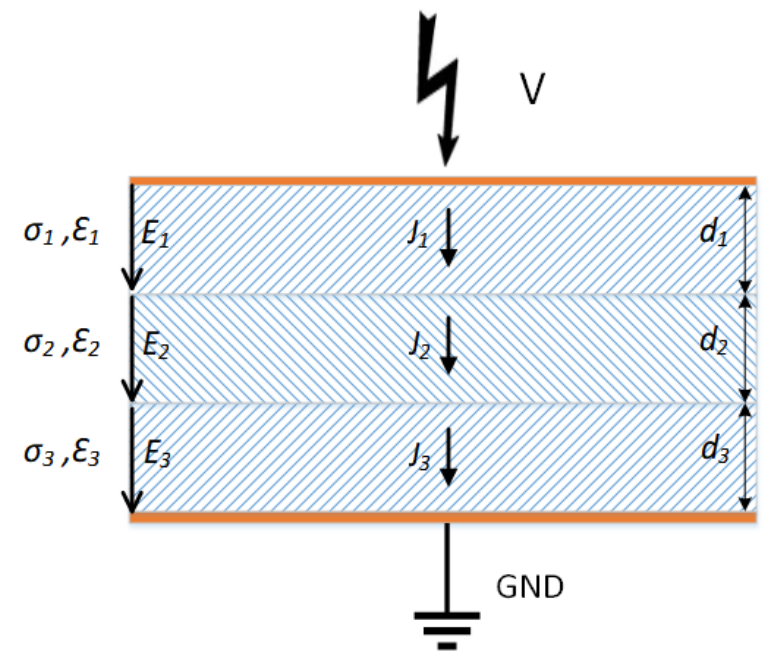

Figure 2-10. An insulation system combined of materials having three different electrical properties

Solving this system will show how the electric field intensity will change over time when an HVDC voltage is applied to this combined insulation system.

As an example, Moser and Dahinden in Weidmann's Transformer-board book present typical values for permittivity and conductivity of the oil and the oil saturated transformer board and how the applied voltage waveform modifies the voltage distribution between two metal electrodes of configuration 2, Table 2-1 [24]. 
Table 2-1. Example of $100 \mathrm{kV}$ AC versus DC voltage drop over a combined oil paper insulation system

\begin{tabular}{|c|c|}
\hline DC at the instant of switching (AC) $100 \mathrm{kV}$ & DC steady state $100 \mathrm{kV}$ \\
\hline upper and lower layers $9 \mathrm{~mm}$ transformer oil & Middle layer $4 \mathrm{~mm}$ transformer board barrier \\
\hline $\begin{array}{c}\text { Relative permittivity } \varepsilon r \\
\text { Mineral oil 2.2, Transformer board } 4.4\end{array}$ & $\begin{array}{c}\text { DC conductivity } \sigma(\mathrm{S} / \mathrm{m}) \\
\text { Mineral oil } 10^{-13} \text {, Transformer board } 10^{-15}\end{array}$ \\
\hline$E_{i}=\frac{V}{\varepsilon_{i}\left[\frac{d_{\text {oil }}}{\varepsilon_{\text {oil }}}+\frac{d_{\text {board }}}{\varepsilon_{\text {board }}}+\frac{d_{\text {oil }}}{\varepsilon_{\text {oil }}}\right]} \quad i=1,2,3$ & $E_{i}=\frac{V}{\sigma_{i}\left[\frac{d_{\text {oil }}}{\sigma_{\text {oil }}}+\frac{d_{\text {board }}}{\sigma_{\text {board }}}+\frac{d_{\text {oil }}}{\sigma_{\text {oil }}}\right]}$ \\
\hline $\begin{array}{l}\text { Voltage over } 3 \text { layers of insulation (kV) } \\
\qquad 45|10| 45\end{array}$ & $\begin{array}{l}\text { Voltage over } 3 \text { layers of insulation (kV) } \\
\qquad 2|96| 2\end{array}$ \\
\hline $\begin{array}{l}\text { Electric field over } 3 \text { layers of insulation }(\mathrm{kV} / \mathrm{mm}) \\
\qquad 5|2.5| 5\end{array}$ & $\begin{array}{l}\text { Electric field over } 3 \text { layers of insulation }(\mathrm{kV} / \mathrm{mm}) \\
\qquad 0.2|24| 0.2\end{array}$ \\
\hline
\end{tabular}

Undoubtedly it is thus interesting to consider that how much the electric fields can deviate when the applied AC voltage is changed to a DC form. The electric field distribution at the instant of switching will be based on a capacitive division and finally based on a resistive division. During the time, before reaching the steady state condition which takes nearly 2 hours for this example, the voltage distribution will be according to Figure 2-11a. The electric field inside oil layers decreases from the initial value of $5 \mathrm{kV} / \mathrm{mm}$ to the final value of $0.2 \mathrm{kV} / \mathrm{mm}$. At the same time the electric field inside the board increases from the initial value of $2.5 \mathrm{kV} / \mathrm{mm}$ to the final dramatic value of $24 \mathrm{kV} / \mathrm{mm}$. It is obvious that a transformer designed to withstand 100kV AC will never withstand, if the same DC voltage is applied to it.

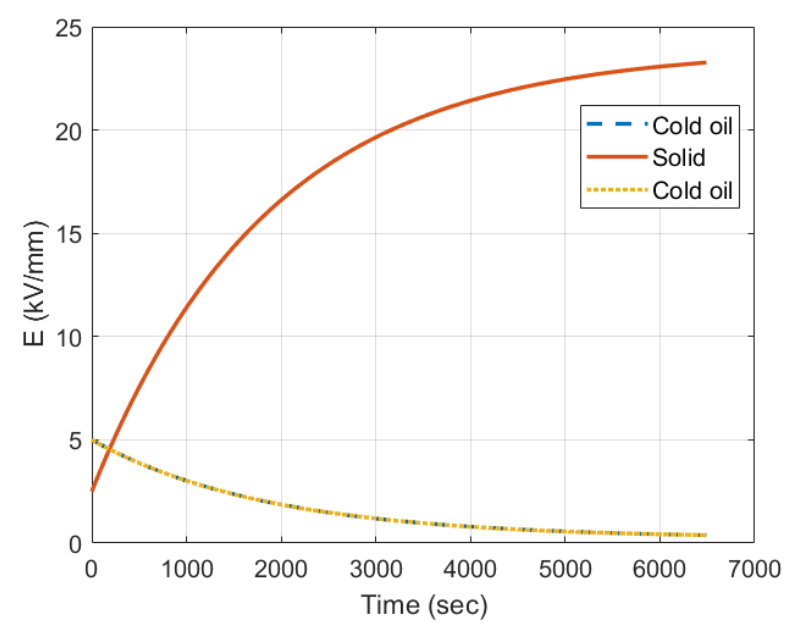

(a)

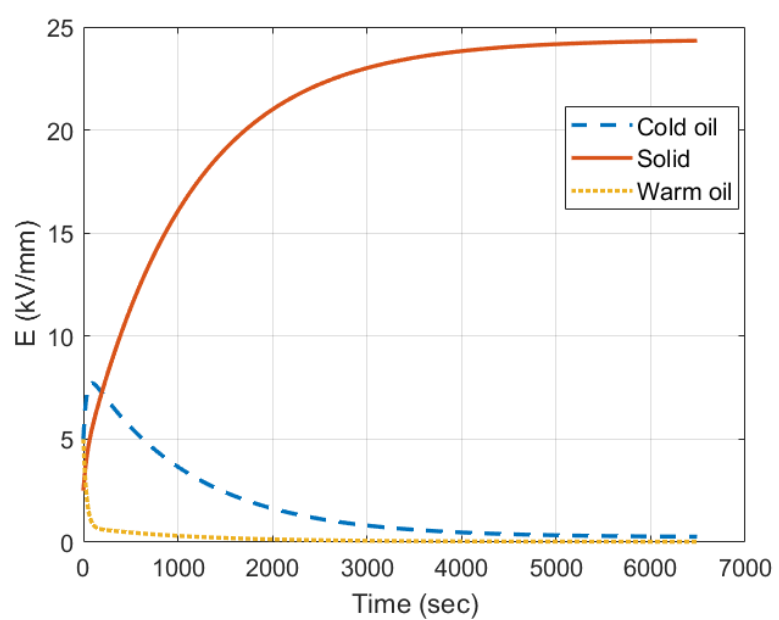

(b)

Figure 2-11. Electric field during transient state inside two similar oil layers and one transformer board layer; Two similar oil and a solid insulation layer electric field distribution under $100 \mathrm{kV} \mathrm{DC} \mathrm{(a),} \mathrm{A} \mathrm{cold} \mathrm{oil,} \mathrm{a} \mathrm{solid} \mathrm{and} \mathrm{a} \mathrm{warm} \mathrm{oil} \mathrm{insulation}$ layer electric field distribution under $100 \mathrm{kV} \mathrm{DC} \mathrm{(b)}$

The situation becomes even more complicated if for example, the conductivity of one of the oil layers has a slight difference from the conductivity of the other layer. Figure 2-11b presents an example, when this difference is in the order of 10 , which is not unusual and can be a result of different temperature, humidity, or even for the oil of the same type, a different production process [65]. Such a condition can happen when during a sudden load change the temperature of the oil near the winding becomes higher than the temperature of the oil in the successive oil ducts (DC conductivity of $10^{-12}$ instead of $10^{-13}$ for example) or when the paper layers having more distance from the winding are colder, have a lower conductivity and experience more electrical field stress. The electric stress inside the oil layer goes higher than the value which is supposed to be highest at the moment of applying the voltage $(5 \mathrm{kV} / \mathrm{mm}$ based on a capacitive division) and reaches a top value of $7.8 \mathrm{kV} / \mathrm{mm}$. 


\subsubsection{Creepage under HVDC stress}

Creepage stress under the HVDC regime should be prevented by a proper overlapping of insulation layers. The barrier arrangements will affect the distribution of the equipotential lines on the neighbouring interfaces. A uniform distribution of tangential electric field is favourable. However, in some cases a non-uniform distribution can increase the DC creepage strength (see Figure 2-12).

Figure 2-12 demonstrates a space between two horizontal parallel electrodes. The upper electrode is charged with a positive HVDC and the lower electrode is connected to ground.

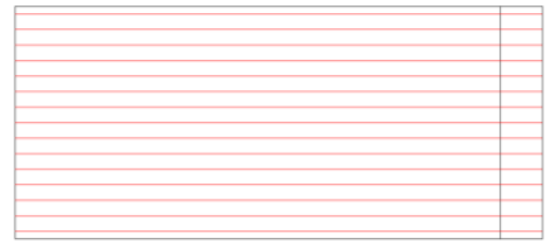

$a 1$

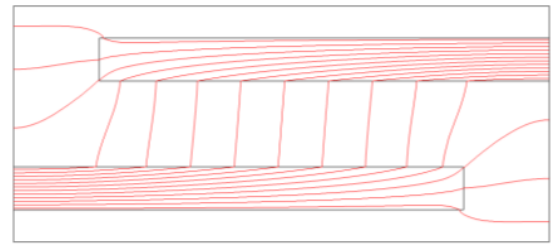

b1

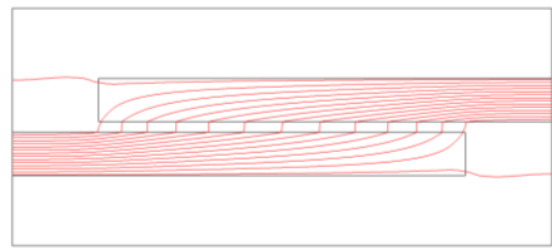

c1

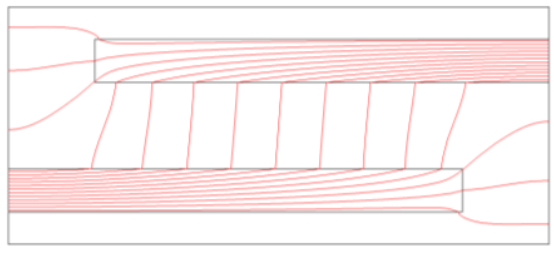

d1

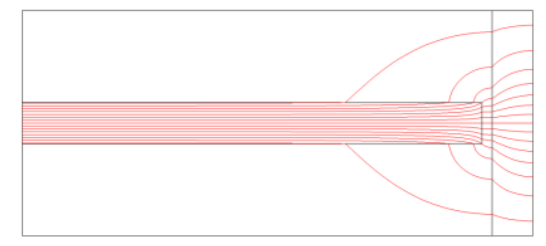

a2

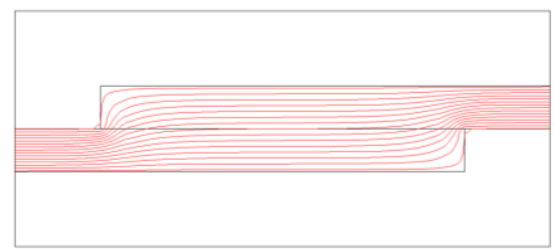

b2

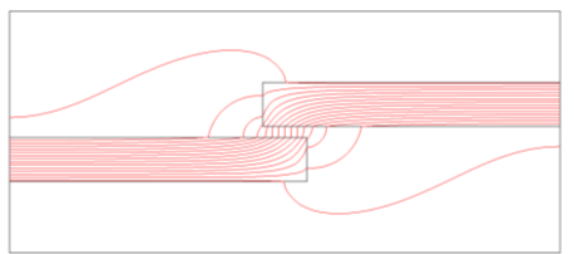

c2

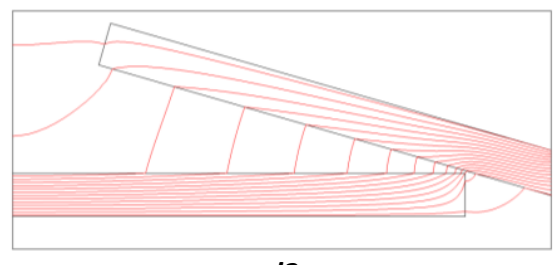

d2

Figure 2-12. Effect of physical positioning of pressboards on creepage stresses on the interfaces: Equipotential lines are shown, the two horizontal parallel electrodes are supplied with DC voltage, the space between electrodes are filled with transformer oil

In figure a1, positioning of a horizontal barrier causes a non-uniform creepage field distribution on the interface of the vertical barrier. Because of higher creepage stress in the middle of the barrier, at a first glance this seems unfavourable. However, the results of experimental creepage discharge tests unveil the positive effect of reduction of the vertical stress at the positions where the barrier meets the electrodes. The test results show a higher creepage withstand for the a2 setup compared with the a1 one [20]. A comparison of figures b1 and b2 shows how an overlap (which is very normal to be used for an $A C$ insulation design) causes a very un-uniform voltage distribution on the barriers' interface which can lead to a creepage discharge.

Figures $\mathrm{c} 1$ and $\mathrm{c} 2$ demonstrate how a reduction in the overlapping area can lead to a very high creepage stress in the boards' surface and spreads even onto the vertical surfaces. At figures $\mathrm{d} 1$ and $\mathrm{d} 2$, the angle between the boards causes a non-uniform voltage distribution which in turn can exceed the maximum permissible DC creepage strength of the OIP-oil interface. 
The creepage distances could be treated by averaging as done in case of AC. However, because of an unknown charge carrier related process it is recommended to hold a high safety factor for instance 2 . Averaging length in case of creepage strength under $A C$ voltage will be from a point on the surface of the solid dielectric having the electrical field higher than a threshold value of $0.1 \mathrm{kV} / \mathrm{mm}$ [22] to the point the strength drops down to a value lower than the threshold value. (Note: In some point the tangential field changes the polarity from positive to negative.)

Experimental creepage breakdown test results which are presented by Ebisawa [20] can be used as reference to extract acceptance criterion for allowable DC creepage stress. The reference gives DC creepage breakdown voltages and strength of three different test setups. As a rule of thumb, a low breakdown probability requires much lower stress levels, at least having a factor of two. For a simple model (a surface between two electrodes), the DC creepage strength values are half the AC breakdown value of an oil gap of the same length. The stresses are strongly dependent on oil and OIP properties and on the field distributions caused accordingly. The breakdown results might be dependent on the individual material properties during the tests. It is also shown that where the bare electrodes are involved the breakdown strength is half of the one where the creepage is on a surface with no direct contact to bare electrodes.

\subsubsection{DC insulation design and non-linear conductivity}

Four main methods to analyse the effect of conductivity of the insulation material for the purpose of DC insulation design are presented in Figure 2-13.

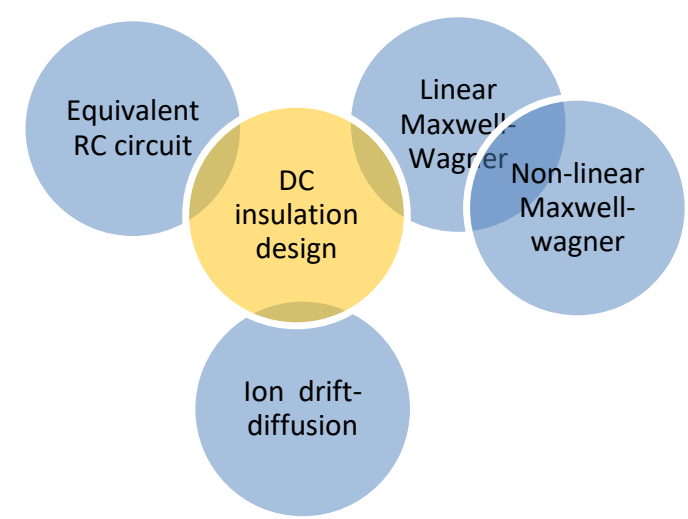

Figure 2-13. Different modelling methods for the study of insulation system under DC voltage stress

\subsubsection{Linear Maxwell-Wagner method}

In the simplest case, the Maxwell-Wagner (MW) model describes the current flow in a simple structure formed by two dielectrics with finite electric conductivities when a DC voltage is applied. It is simple and can predict the dynamics of charge transport as well as interfacial charge accumulation which affect the resulting field distribution in the insulation system. The equations describing the process and included in the MW model are

$$
\begin{array}{ll}
\mathbf{E}=-\nabla U & \\
\mathbf{J}=\sigma \mathbf{E} & \text { Ohm's law } \\
\nabla \cdot(\varepsilon \mathbf{E})=\rho & \text { Gauss's law } \\
\nabla \cdot \mathbf{J}=-\partial \rho / \partial t & \text { Continuity equation } \\
d \rho_{s} / d t=J & \text { Surface charge accumulation }
\end{array}
$$


where $U$ is the applied potential distribution; $\mathbf{E}$ and $\mathbf{J}$ are the vectors of the electric field and current density, respectively; $\sigma$ is the electrical conductivity; $\varepsilon$ is the dielectric permittivity; and $\rho$ is the space charge density, $\rho_{s}$ is the interfacial charge density and $J$ is the current density

According to the Linear MW model (LMW) after applying a step DC voltage to the oil/paper dielectrics, the initial electric field distribution is based on material dielectric constants ratios $(2.2$ for oil and 4.4 for paper for example), and therefore the electric field intensity in the oil has a value nearly twice its value inside the paper. But at the final steady state, which may last up to several hours, it is the conductivity of materials that determines stress; a material with greater conductivity will take larger field intensity, and vice versa [66]. Since the ratio of the oil to paper conductivity may vary from 10 to 1000 or higher [67] and [52], the paper will be more stressed than the oil at steady state. Therefore, the conductivity plays a crucial rule in an insulation system which is stressed by a HVDC voltage.

\subsubsection{RC network modelling based on equivalent electrical circuit elements}

One can simulate an insulation system using equivalent electrical circuit elements. Liebschner has used this method to describe the temperature and voltage dependent behaviour of different combinations of oil-paper insulation systems [68]. A simple example is a two-layer oil-paper system where a step voltage is applied between two bare electrodes (see Figure 2-14).

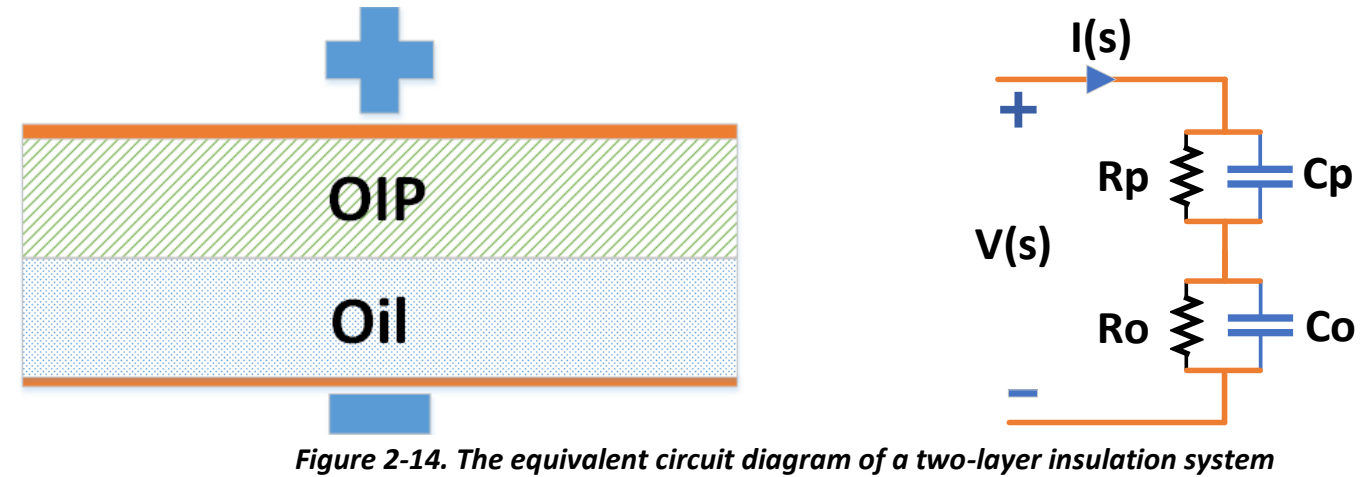

In this figure, $R_{p}$ and $R_{o}$ are the equivalent resistances of OIP, and oil and $C_{p}$ and $C_{o}$ are the equivalent capacitances of OIP and oil respectively. In a normal case $\sigma_{\mathrm{p}}$ is much bigger than $\sigma_{0}$, where $\sigma$ is the conductivity of the insulation layer. The following well known equations relates the physical properties of the insulation layers to the elements of the equivalent circuit.

$$
\begin{aligned}
& C=\varepsilon_{0} \varepsilon_{r} \frac{A}{d} \\
& R=\frac{1}{\sigma} \frac{d}{A}
\end{aligned}
$$

Assuming a zero state for the circuit (zero set initial condition) and using a system of first order differential equations, the voltage and currents of the elements can be calculated having

$$
V_{p}=\frac{C_{o}}{C_{p}+C_{o}} V_{s} e^{-t / \tau}+\frac{R_{p}}{R_{p}+R_{o}} V_{s}\left(1-e^{-t / \tau}\right)
$$

where

$$
\tau=\frac{R_{p} R_{o}}{R_{p}+R_{o}}\left(C_{p}+C_{o}\right)
$$

This method assumes having a constant (not variable with time or the applied voltage for example) conductivity values for the oil and OIP which is not true. However, the method is a simple tool for preliminary insulation design activities. 


\subsubsection{Charged particles generation, drift and diffusion method}

In 1980, ASEA researchers proposed a new model for simulation of conductivity of the transformer oil, named ion drift-diffusion. The current transport equations, the continuity equations and the Poisson equation are adopted in this model. This model can predict the density of the ions of different polarities as a function of time and their position. ABB later has used the Kerr electrooptical measuring technique to verify the model. It was shown by experiments that the oil has a nonlinear behaviour, especially at lower electric fields [14].

In [69] it is stated that a higher electric field intensity near the bare electrodes cannot be evaluated by the conventional MW method, in which the electric fields are estimated using the materials conductivities. Therefore, a charge carrier-based model using the Poisson-Nernst-Plank (PNP) equations is used. This model can predict the charge accumulation in front of the electrodes as well as the solid dielectric barriers. This model considers the charges injection from electrodes, oil intrinsic charge, charges movement inside the oil due to Coulomb forces and the diffusion process, recombination of charges and also their absorption into electrodes. Various combinations of oil/paper dielectrics are investigated to find electric field distribution using this method. But the findings are so different from the MW model results which is based on the materials conductivities ratio. But the PNP model parameters like ion mobility and its dependence on oil contamination, the charge injection rate and its dependence to electric field intensity and polarity [70] and [71] are not fully known and determined yet. It can be deduced from the results reported in [69] that at the final state after applying HVDC, the electric field intensity inside the paper obtained from the PNP model is somewhat lower than what is obtained from the MW model. This fact suggests that the stress in the paper will be overestimated in the MW model. Therefore, if we use the MW model to determine the geometry of the insulation, the safety factor for the paper will be satisfactorily high enough.

\subsubsection{Non-Linear Maxwell-Wagner Method}

After explaining the physical phenomena of the non-linear dependence of the oil conductivity to change in applied voltage, temperature, etc. it is explained why the Maxwell Wagner method is still a reliable tool for insulation design on an HVDC transformer. However, in a Linear Maxwell-Wagner (LMW) model, the electrical parameters of the oil and paper are supposed to be constants. Although this assumption is nearly true for permittivity, it is known that conductivity is highly dependent on different parameters like temperature, electric field, water content, time, Oil impregnated Pressboard (OIP) density, electrode materials and their dielectric coating and cover $[52,72,73]$.

To consider the nonlinear dependence of the oil and OIP to the applied voltage and temperature, in this work a Non-Linear Maxwell-Wagner (NLMW) method is presented and employed. This is crucial to be able to design a compact transformer, and thus accurate calculations of the dynamic behaviour of the electric fields will be done. The NLMW model is adopted for MFT insulation design in this report, which accounts for (at least) field and temperature dependencies of the electric conductivity of the constituting materials and its results is compared with LMW.

\subsubsection{Effect of covering the electrodes}

According to Weidmann design curves, for an AC design, the bare metal electrodes should be coated with solid insulation [63] to increase the total withstand strength and to prevent partial discharge originating from sharp corners. In contrary to AC voltage applications, for DC voltage there should be a pass for to the leakage current. This means that an insulation cover should not totally cover all the electrodes [65]. 
Figure 2-15 demonstrates how covering of high voltage electrode - which is common for a safe AC insulation design - can cause a very high stress on the covered OIP and lead to an unwanted discharge inside it. At Figure 2-15a, the charge carries have a pass to flow and a resistive voltage division based on the conductivities of the materials involved is in place. At Figure 2-15b, the current pass is barred. Therefore, the voltage-drop over the oil gap is zero and all the voltage difference between the electrodes stresses the covering barrier. This can lead to a stress much higher than the withstand stress of covering barrier.

Investigations made by Okubo et al. [19] and Gabler et al. [74] show that there is more charge injections from the cathode compared to the anode. According to Okubo, the electric field near the cathode drops down much faster compared to the field near the anode. According to Gabler the setup with a covered cathode has a higher DC breakdown voltage. The reason might be that the injected electrons from the cathode move inside the oil and accumulate on the surface of the OIP (negative charge affinity). This leads to a lower stress at the cathode side and a higher stress at the anode side. As a direct result, the LV winding of the transformer as well as the grounded parts should be covered for a higher isolation purpose.

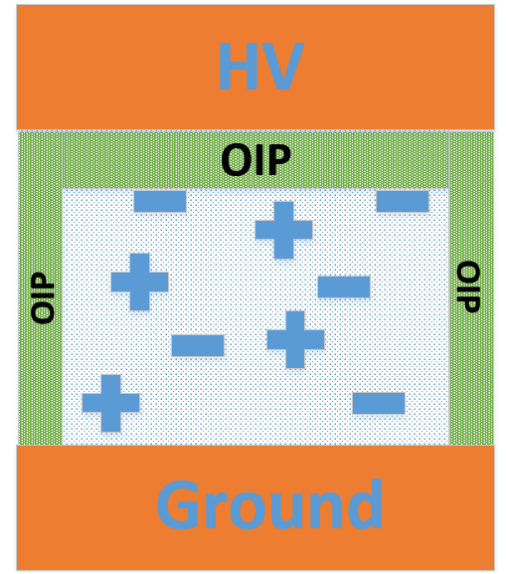

(a)

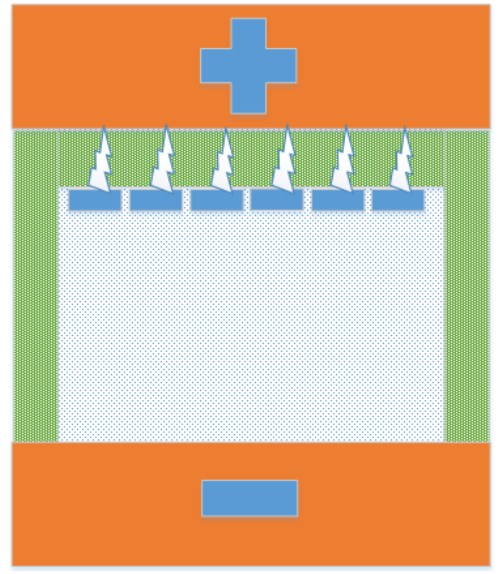

(b)

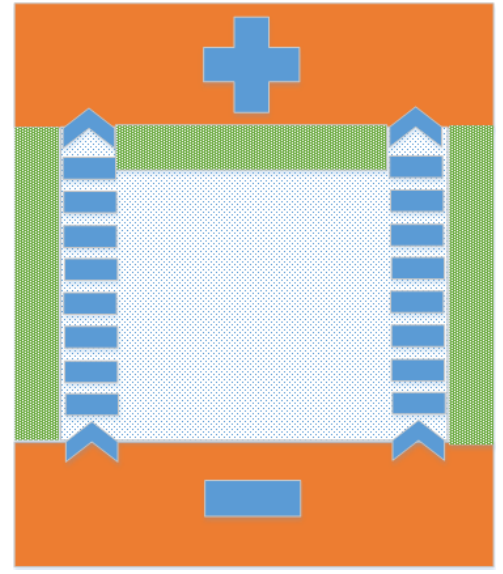

(c)

Figure 2-15. The effect of covering electrodes on the discharge in the insulation layer; An oil/OIP system between two electrodes not supplied by voltage (a), Positive electrode totally covered by OIP (b), Positive electrode partly covered by OIP (c)

\subsection{Conductivity measurements in time domain}

If a step DC voltage is applied to a liquid or solid sample (see Figure 2-16a), the current directly after application of voltage is mainly a superimposed polarisation and conduction current for solids and conductive for liquids. After a relatively long time the polarisation process ends and a current based on the conductivity of insulation material will flow (see Figure 2-16b). After short circuiting the electrodes (removing the voltage source), only a depolarisation process will take place (see Figure 2-16a), resulting in a discharge current, slowly declining towards 0 . 
$U_{c}$

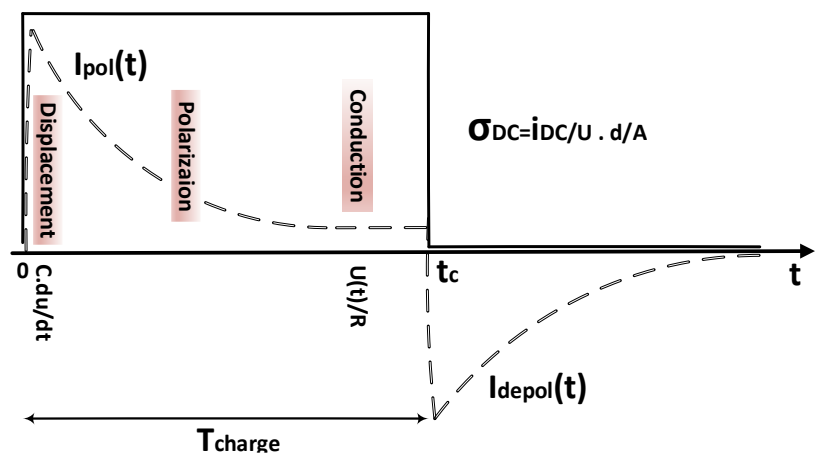

(a)

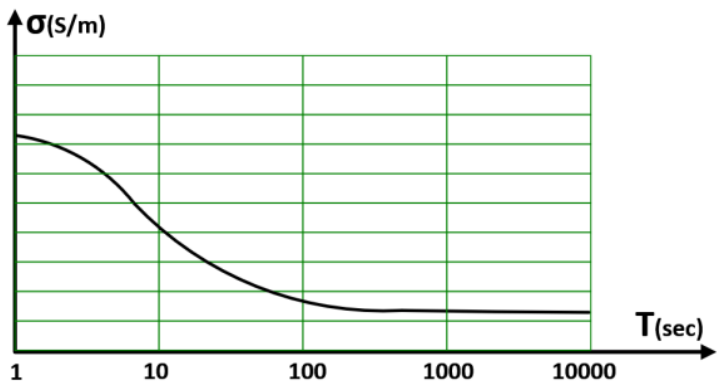

(b)

Figure 2-16. Polarisation, Depolarisation Current (PDC) measurement concept; Applied step DC voltage, short circuiting the object, and the resulting polarisation, conduction and depolarisation currents (a), Conductivity versus time of application of step voltage (b)

For a new HVDC transformer application, the oil and OIP conductivities measured at different conditions (depending on transformer operation condition after energisation) should be used for the insulation design of a transformer (see Table 2-2). The transformer design should be repeatedly checked at different physical positions in the tank, applied voltage, time of application of voltage and loading conditions. In addition, for a transformer in service, the humidity, particle contamination, aging or the thermodynamic oil flow, have considerable effects on the conductivity figures and consequently on the predicted stresses on an HVDC transformer. However, these effects are not considered in the scope of this study.

Table 2-2. Different conditions during oil conductivity measurements

\begin{tabular}{|c|c|c|c|}
\hline Conductivity & $\begin{array}{c}\text { At the instant of } \\
\text { energization }\end{array}$ & $\begin{array}{c}\text { After energization and before } \\
\text { loading }\end{array}$ & $\begin{array}{c}\text { At steady state after long time of } \\
\text { loading }\end{array}$ \\
\hline Time & Short time & Relatively long & Long \\
\hline Temperature & environment temp. & Low & Medium or High \\
\hline
\end{tabular}

\subsubsection{Conductivity measurement system set-up}

Many standards have presented methods for surface or volume conductivity of liquid or solid insulation materials (IEC 61620 1998, IEC 60247 2004, ASTM D1169 2011, IEC 62631-3-1\&2 2016 or ASTM D257 2014). A comparative table is prepared, and the strong and weak points of the measuring methods are summarized in a Cigré publication [52]. The result of this comprehensive study shows that none of these standard methods guarantee both reproducible and comparable results with the conductivities under HVDC test or service conditions. However, the measurement method proposed in this report refers to the guidelines from the same standards to be implemented at different measurement stages. To perform a reliable conductivity measurement procedure the following should be considered:

- Test object cell and temperature chamber

- Current measuring and data acquisition system and its protection

- High voltage application system and safety

- Mitigation of noises

- Preparation and testing of oil samples

- Preparation and testing of solid insulation samples

\subsubsection{High voltage conductivity measurement test cell}

The test cell for performing the measurements was essentially a guarded three-electrode system and the same as introduced in IEC 93, 1980 \& IEC 62631-3-1, 2016 [75] with some improvements 
suggested in Cigré report 646 [52] to make it suitable for measurements at higher stress voltages (see Figure 3-2). The middle part has been manufactured with two different sizes, one having $D=97.8$ $\mathrm{mm}$ with $R 2=1 \mathrm{~mm}$ [52] and to handle higher voltages, one having $D=99,8$ with $R 2=2 \mathrm{~mm}$ [76]. The other rounding dimensions are $R 1=1 \mathrm{~mm}, R 3=3 \mathrm{~mm}$ and $R 9=5 \mathrm{~mm}$.

To calculate the volume conductivity of the insulation, the effective area of the guarded measuring electrode can be calculated from the following equations considering precautions explained by Lisowski [77] and given by ASTM D257-14 [78]

$$
A_{e f f}=\pi \frac{\left(d_{1}+B g\right)^{2}}{4}
$$

where $d_{1}$ is the diameter of the measuring electrode $(99.8 \mathrm{~mm}), g$ is the gap width between the measuring and guard electrodes $(1.1 \mathrm{~mm})$, and $B$ is the factor for accounting for the diameter enhancement by spreading the electrode margin

$$
B=1-\frac{4}{\pi} \frac{h}{g} \ln \left(\cosh \left(\frac{\pi}{4} \frac{g}{h}\right)\right)
$$

\subsubsection{OIP conductivity values calculation using charge difference method}

If a DC step voltage was applied to a liquid insulation (e.g. mineral transformer oil), a decreasing current was observed in most of the cases. In contrast to solid insulation, this current is dominated by the conduction current component, and the depolarization current component is negligible from the very beginning (after a few seconds). Thus, the conductivity was calculated from the ratio of the polarization current and the voltage. Yet, the result was time-varying and strongly dependent on many other parameters (e.g. electrical stress and temperature). In the case of using oil impregnated pressboard, the conduction current was low, and the polarization current was relatively high. Therefore, the charge difference method [79] was used, which is less sensitive to noise signals and gives a good estimate of the conductivity value in short measuring times. In this method, the following governing equations are utilized:

$$
\begin{aligned}
& Q_{p o l}(t)=\int I_{p o l}(t) d t \\
& Q_{\text {depol }}(t)=\int\left|I_{\text {depol }}(t)\right| d t \\
& Q_{\text {dif }}(t)=Q_{p o l}(t)-Q_{\text {depol }}(t) \\
& Q_{\text {dif }}(t) \approx a t+b \\
& \sigma=\frac{a}{E A_{\text {eff }}}
\end{aligned}
$$

where, $I_{p o l}$ and $I_{\text {depol }}$ are the polarization and depolarization currents respectively, $Q_{p o l}$ and $Q_{\text {depol }}$ are the polarization and depolarization cumulative charges, $Q_{\text {dif }}$ is the charge difference, $a$ is the slope of the $Q_{\text {dif }}$ curve, $A_{\text {eff }}$ is the effective area of the measuring electrode and $E$ is the applied field intensity.

\subsubsection{Using the Arrhenius law}

At lower temperatures, the stabilization of the current can take long time. In addition, the signal to noise ratio can be unacceptably low. To achieve the conductivity values in these conditions, the measurements can first be done at $50{ }^{\circ} \mathrm{C}$ and $90{ }^{\circ} \mathrm{C}$ and later the conductivity in $30{ }^{\circ} \mathrm{C}$ can be calculated according to Arrhenius law [5] 


$$
\sigma_{D C}(T)=\sigma_{D C, r e f} e^{-\frac{W_{a}}{k T}}
$$

where, $\sigma_{D C \text {,ref }}$ is the reference value of conductivity at two different temperatures, $T(\mathrm{~K})$ is the target temperature, $W_{a}(\mathrm{~J})$ is the activation energy, and $k(\mathrm{~J} / \mathrm{K})$ is the Boltzmann's constant (see Figure 3-16 as an example of application of this method).

\subsection{MFT's important magnetic design concepts}

The duty cycle of the applied rectangular wave, $D$ is assumed to be 0.5 , which is defined as

$$
D=\frac{t_{\text {on }}}{T}
$$

where $T_{\text {on }}$ is the conduction (on) time of the switch and $T$ is the time period of the wave.

The relative rise time of the wave as is assumed to be 0 for the design, which is defined as

$$
R=\frac{t_{\mathrm{r}}}{T}
$$

where $t_{r}$ is the rise time of the wave.

Core cross section calculated for square wave shape can be calculated as

$$
\begin{aligned}
& V=N \frac{d \varphi}{d t} \\
& \varphi=\frac{1}{N} \int_{0}^{T} v d t
\end{aligned}
$$

and

$$
\varphi_{m}=\frac{V_{D C} D^{T} / 2}{N}=\frac{V_{D C}}{4 N f}
$$

and

$$
A_{c}=\frac{\varphi_{m}}{B_{m}}=\frac{V_{D C}}{4 N f B_{m}}
$$

where $B_{m}$ is the maximum working flux density of the core (nearly $80 \%$ of the saturation flux density), $K_{c}$ is the core filling factor (the ratio of effective to physical cross sections which is 1 for Ferrite), $N$ is the number of turns for a winding and $f$ is the switching frequency. $A_{c}$ is the effective cross section of the transformer core and $\varphi_{m}$ is the amount of flux in the core.

Core cross section calculated for sinus wave shape can be calculated as

$$
A_{c}=\frac{V_{r m s}}{4.44 N f B_{m}}
$$

\subsubsection{Leakage inductance}

The voltage wave forms applied to the MFT in a DAB converter are not sinusoidal as in a conventional low frequency transformer (see Figure 2-1b). Therefore, in case of this type of transformer, the leakage inductance and its related phase shift are under consideration and the transformer leakage inductance, $L_{\sigma}$ corresponds to the value calculated according to

$$
L_{\sigma}=V_{D C 1} V_{D C 2} \phi_{\min }\left(\pi-\phi_{\min }\right) /\left(2 P_{\text {out }} \pi^{2} f_{s} n\right)
$$


where, $P_{\text {out }}$ is the output power of the DAB converter, $V_{D C 1}$ is the $D C$ voltage of the LV side of the DAB converter, $V_{D C 2}$ is the DC voltage of the HV side of the DAB converter, $n$ is the turns ratio of the transformer, $f_{\mathrm{s}}$ is the switching frequency and $\varphi_{\min }$ is the phase shift between the bridges which should be higher than a predetermined value to achieve Zero Voltage Switching (ZVS) [34].

\subsubsection{Calculation of leakage inductance of axisymmetric MFTs}

A method based on an analytical solution of the Poisson equation in planar coordinates is developed and presented in [80]. As a more accurate calculation procedure for the leakage inductance in highpower shell-type MFTs with round windings considering the effect of the core structure one can use the analytical method proposed by Robbins in 1956 which was originally derived for calculating the power transformer's leakage inductance in cylindrical coordinates [40]. In his method, the core window is assumed to be endless from its outer side as is the case for large power transformers. In the method, the current density inside the core window is written as a single Fourier series as a function of core window height. The magnetic potential is then represented with a series expansion including modified Bessel and Struve functions. This method is suitable when the effect of curvatures of the windings needs to be taken into account in the leakage inductance calculation. Robbins' method seems to be a suitable method to be adopted for shell-type power electronic transformers such as MFTs with polygonal core structure.

In this analytical method first the solution space is divided into different regions. Maxwell's equations are used to obtain

$$
\nabla^{2} \mathbf{A}=-\mu_{0} \mathbf{J}
$$

where $\mathbf{A}$ is the magnetic vector potential and $\mu_{0}$ is the permeability of air. The current density vector $\mathbf{J}$ is azimuthal and because of the axisymmetric geometry, all the field quantities are independent of the $\varphi$ coordinate.

With these assumptions, (2-33) becomes in cylindrical coordinates

$$
\frac{\partial^{2} A}{\partial r^{2}}+\frac{1}{r} \frac{\partial A}{\partial r}-\frac{A}{r^{2}}+\frac{\partial^{2} A}{\partial z^{2}}=-\mu_{0} J
$$

The current density could be written as a Fourier series in terms of a fundamental spatial period of length $L$, or window height as

$$
J=J_{0}+\sum_{n=1}^{\infty} J_{n} \cos \left(\frac{n \pi z}{L}\right)
$$

For one coil with a constant current density $j$,

$$
\begin{aligned}
& J_{0}=j \frac{\left(z_{2}-z_{1}\right)}{L} \\
& J_{n}=\frac{2 j}{n \pi}\left[\sin \left(\frac{n \pi z_{2}}{L}\right)-\sin \left(\frac{n \pi z_{1}}{L}\right)\right]
\end{aligned}
$$

Substituting the Fourier series (2-35) into (2-34), $A$ will be obtained as

$$
A=\sum_{n=0}^{\infty} R_{n}(r) \cos (m z), m=\frac{n \pi}{L}
$$

Using the solution of $A$ inside the core window, the self and mutual inductances of a coil system can be obtained. The mutual inductance between coil 1 and coil 2 with uniform current densities $I_{1}$ and $I_{2}$ is given by

$$
L_{12}=\frac{1}{l_{1} I_{2}} \int_{V_{1}} \mathbf{A}_{2} \cdot J_{1} d V_{1}
$$


Considering two coils (with zero net ampere-turns) inside the core window, the leakage inductance can be calculated using the total magnetic energy stored inside the core window. The magnetic energy stored in the system is obtained by

$$
W=\frac{1}{2} \sum_{i=1}^{2} \sum_{j=1}^{2} L_{i j} I_{i} I_{j}
$$

where $L_{i j}$ is the mutual inductance of coils $i$ and $j$, calculated using (2-39).

Once the total energy is obtained, the leakage inductance can be calculated as

$$
L_{\text {leakage }}=\frac{2 \mathrm{~W}}{I^{2}}
$$

where $I$, denotes the current in the side from which the leakage inductance is calculated.

\subsubsection{Transformer losses}

\subsubsection{Core losses}

Basically, three methods have been developed for magnetic material's loss calculations [3]. First, the loss separation method, which includes calculation of static hysteresis losses, classic eddy current loss and excess eddy current loss and is usually too complicated to be used for engineering tasks (except in Fem analysis). Hysteresis loss is independent of the wave shape and it is frequency and flux density dependent. This loss component can be calculated and verified by measurements. The classic eddy current loss can also be calculated but the excess eddy current loss should be included which is a function of frequency and maximum flux density, which needs to be determined by a series of measurements and parametrizations for the investigated magnetic material [81]. Second is the time domain approach which is the method used for loss calculations in FEM [3]. A third strategy is to use a set of empirical methods, all based on empirical measurements and on the formulation made by Steinmetz in 1892 [82]. In this work the samples to be used were available and measurement were chosen to be used for the loss determination.

A few types of core materials are popular among the researchers which work with medium frequency transformer designs. Ferrite and Nanocrystalline cores are previously used by the author to produce two $50 \mathrm{~kW}$ transformers [8]. The 'core losses - frequency' curves of Nanocrystalline cores from their datasheets are normally given at a very low operational working point (0.1-0.3 $\mathrm{T}$ for Nanocrystalline cores compared with 1-1.2 $\mathrm{T}$ as working point flux). In case of Ferrite cores, the data given in the core data sheet does not include the working point flux density of $0.3 \mathrm{~T}$ (see Figure 2-17).

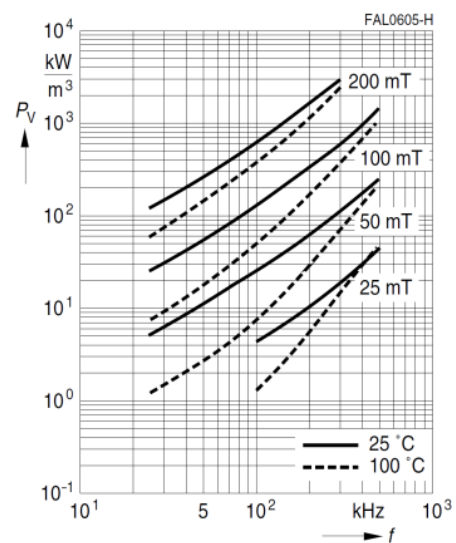

(a)

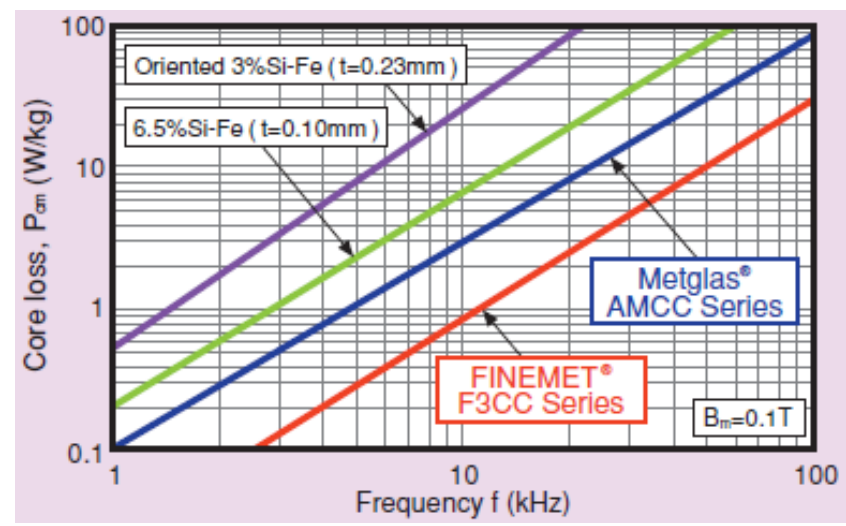

(b)

Figure 2-17. Loss-frequency curves of sample N87 Ferrite and Finemet Nanocrystalline cores; Ferrite core [83] (a), Nano crystalline core [84] (b) 


\subsubsection{Winding losses}

In this work an effective numerical method is utilized and practically verified, for copper loss calculation of the MFTs with windings having Litz wire conductors.

\subsection{MFT's current}

To calculate the current, in the transformer equivalent circuit, it is assumed that the real part of the complex impedance is ignorable compared with the imaginary part i.e. the leakage inductance [85]. With this assumption

$$
I_{p}=\left(V_{p}-V_{s}\right) / X_{L}
$$

where

$$
v_{p}=2 \sqrt{2} V_{i} / \pi
$$

The $h_{t h}$ element of the r.m.s. value current in the primary side of the transformer is calculated from

$$
I_{p h}=\frac{2 V_{i} \sqrt{1-\cos (h \varphi)}}{\pi^{2} f h^{2} L_{s}}
$$

where $V_{i}$ is the bus voltage of the primary, $\varphi$ is the phase shift between the primary and secondary side voltages of the transformer, $f$ is the frequency and $L_{s}$ is the leakage inductance of the transformer.

For an MFT, the leakage inductance seen from the primary side can be calculated from [80]

$$
L_{s}=\frac{2 W}{I_{p}^{2}} M L T
$$

where $M L T$ is the mean length of the winding turns and $W$, the total magnetic energy stored inside the core window can be obtained from

$$
W=\frac{1}{2} \int \mathbf{A} \cdot \mathbf{J} d v
$$

Using Maxwell's equations and choosing the Coulomb gauge, the well-known equation for the vector potential $\mathbf{A}$ is obtained

$$
\nabla^{2} \mathbf{A}=-\mu_{0} \mathbf{J}
$$

where $\mathbf{J}$ is the current density vector in the $z$ direction, and it can be assumed that all the field quantities of it are independent of $z$.

Losses caused by the current in a transformer are named Load losses [36]. Load losses consists of winding Ohmic resistance losses and additional losses. Winding DC resistance is calculated using

$$
R=\rho \frac{l}{A}=\rho(M L T \times N) /\left(\pi r^{2} n_{S}\right)
$$

where $R$ is the resistance of the winding, $\rho$ is the resistivity of the copper wire, MLT is the mean length of the strands in each winding turn, $N$ is the number of the turns of the measurement side, $r$ is the radius of each wire strand, $n_{s}$ is the number of the strands.

The additional or stray losses are produced by AC currents from the leakage field of windings and the field of high-current carrying leads. The additional losses are produced in the windings and structural parts. The losses in the windings are eddy current losses and circulating current losses.

Depending on the frequency, the presence of these additional losses will make AC current losses higher than equivalent DC current copper losses. Circulating current losses can be a result of having parallel conductors of different length or exposed to different electric field which leads to a different 
voltage drop over two parallel conductors of the same length. For the prototype transformer considered in this study parallel conductors are axially ordered and there are no circulating current losses in them. However, because of the relatively high frequency, eddy current losses are present and will be investigated.

Because of the high skin and proximity effects at high frequencies it is not economical to use normal wires to produce the windings. Copper foils are suitable alternatives for higher frequencies, but they need a great care during the production and for the termination when mounted in the transformer. With the introduction of Litz wires (bunch of individually insulated conductors), a higher flexibility in the production and lower losses can be gained.

A Litz wire consists of a group of enamelled strands (see Figure 2-18b). The enamel insulation of the conductor is normally modified polyurethane. At higher frequencies, increasing the number of strands together with the reduction of the strand's diameters, counteract the increase in the conductor resistance. The alternating current causes eddy currents in the conductor which acts to counteract the flow of the fundamental current. At higher frequencies, the effect of these eddy currents increases, and an AC resistance is added to the DC resistance. The eddy current loss in the middle of the conductor has a low value and increases when moving towards the outer part of the conductor. This causes the current to flow closer to the surface of the conductors instead of in the whole conductor (the skin effect). The skin depth [m] is a representation of the thickness of the conductor which is carrying the current and can be calculated from

$$
\delta=1 / \sqrt{\pi f \mu \sigma}
$$

where $\omega$ is the radian frequency $(\mathrm{rad} / \mathrm{s}), \mu$ is the permeability of the conductor $(\mathrm{H} / \mathrm{m})$ and $\sigma$ is the conductivity of the conductor $(1 / \Omega \mathrm{m})$. For example, the skin depth for copper at the frequency of 5 $\mathrm{kHz}$ is $1 \mathrm{~mm}$ and this means that the current in the depth of $1 \mathrm{~mm}$ is reduced to $36.8 \%$ of the one at the surface [36]. Because of the proximity effect, the fields of the adjacent conductors increase the eddy current losses. The cross-sectional area of the single conductor is reduced to minimize these losses. Several conductors in parallel are used to carry the nominal current of a wire. To compensate the effects of the fields on the individual strands, the conductors are twisted together so that, throughout the length of the wire, the position of one conductor transposes regularly. Because of the high capacitance effect of the conductor, Litz wires can be used only up to $2 \mathrm{MHz}$. A typical diameter of each conductor as a function of frequency is $0.4 \mathrm{~mm}$ for $50-1000 \mathrm{~Hz}, 0.25 \mathrm{~mm}$ for $1-10 \mathrm{kHz}$ or $0.032 \mathrm{~mm}$ for 1.5-2.8 MHz [86]. With an effective reduction of the skin effect, Litz wires have the best performance in high frequency circuits. Commercial production of several types and dimensions of the wire gives a good flexibility for getting the highest benefit in this project. Rectangular Litz wires are estimated to be one of the best candidates for usage as the high and low voltage conductors in this project (see Figure 2-18a). This arrangement helps for higher density and more mechanical strength of the winding. The available constructions from one of the leading Litz wires manufacturers are presented in Reference [86]. 


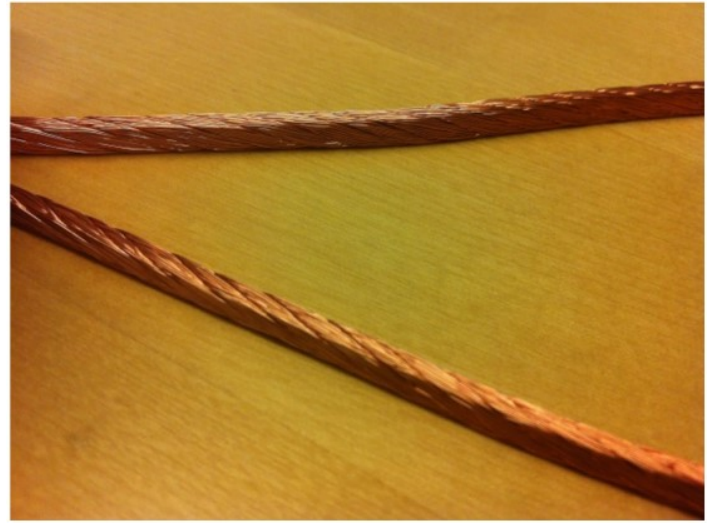

(a)

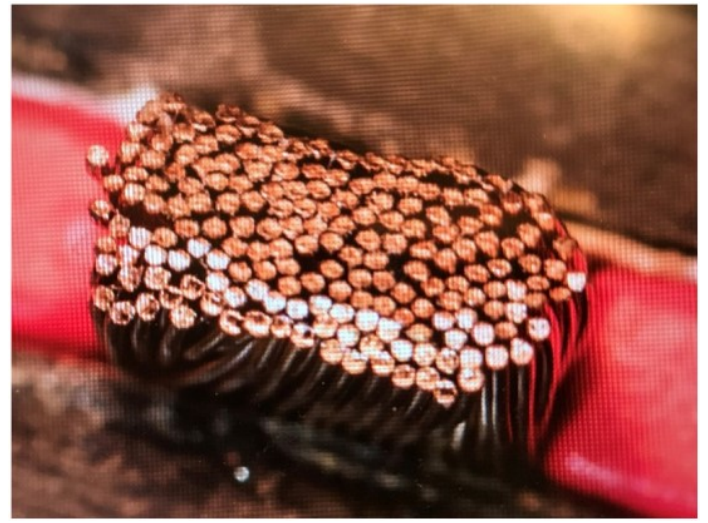

(b)

Figure 2-18. A rectangular Litz wire and its strands; Rectangular Litz wire (a), Bunch of groups of enamelled strands (b)

2.4.2.2.2 Evaluation of winding losses using equivalent permeability and conductivity method One method of calculating eddy current losses including skin and proximity effect losses is to make a finite element model, in which each turn in a winding is explicitly represented. By modelling each turn, the distribution of current density within each turn due to these effects, can be accurately determined. However, modelling every wire individually can be computationally expensive. An alternative approach is to replace a wound region composed by many wires with a region with proper equivalent complex-valued material properties.

Continuum representations of wound coils allow proximity and skin effect losses to be represented in numerical models without explicitly modelling each turn in the coil. Moreau et al. [87] described the use of a complex-valued magnetic permeability for representation of transformer windings with rectangular conductors, presenting closed-form expressions for frequency-dependent permeability. Gyselinck and Dular [88] presented a numerical method for obtaining equivalent properties of a round-wire winding with hexagonal packing. Dowell [45] replaced a winding composed of round wires with an "equivalent" foil winding that admits an analytical solution for proximity losses. Meeker [89] derived approximate but closed-form expressions for the equivalent conductivity and permeability of regions filled with hexagonally packed round wires, allowing proximity and skin effects to be included in 2D AC field computations. In this work the method developed in [89] is used for calculation of eddy current losses of MFT windings made by Litz wires.

The effective material properties of the wound region can be expressed as [89]

$$
\begin{aligned}
& \mu_{\text {eff }}=(1-c) \mu_{0}+c \mu_{f d} \\
& \sigma_{\text {eff }}=\frac{\sigma f i l l}{\frac{\mu_{0}}{\mu_{f d}}+\frac{(1-c)}{c} j \Omega-\frac{1}{3} \frac{\mu_{\text {eff }}}{c \mu_{O}} j \Omega}
\end{aligned}
$$

where the parameter $c$, representing the fill factor of the equivalent foil geometry, is defined as

$$
c=\sqrt{\frac{2 \sqrt{3}}{\pi} \text { fill }}
$$

where fill is copper fill factor and the non-dimensional frequency is defined as

$$
\Omega=\frac{\sqrt{3} \pi c \omega \sigma \mu_{0} R^{2}}{8}
$$

and the frequency-dependent permeability of a single equivalent foil is

$$
\mu_{f d}=\frac{\mu_{0} \tanh \sqrt{j \Omega}}{\sqrt{j \Omega}}
$$




\subsection{Characterisation for high frequency stress}

During a long operation time for a high-voltage equipment under high frequency stress, both the voltage and the heat contribute to the degradation of the insulation material.

\subsubsection{Voltage stress}

Any insulation material has a withstand strength which is dependent on the applied voltage rate of rise, magnitude and duration. Three examples of voltage wave shapes which are standardized during the development of the power transformer's insulation design are AC at power frequency $(50 \mathrm{~Hz})$, Lightning Impulse (LI) and Switching Impulse (SI). As an example, for testing a power transformer, a switching impulse voltage can have a time to peak value down to $100 \mu \mathrm{s}$, a magnitude or peak value of $375 \mathrm{kV}$ and a duration (time duration while the magnitude is higher than $90 \%$ of the peak value) of $200 \mu \mathrm{s}$ and time to zero of $2500 \mu \mathrm{s}$ [90]. To design an insulation system for a power transformer, the Design Insulation Levels (DIL) should be determined first [36].

The following can be considered as additional sources for overvoltage stresses on the MFTs, which are in addition to the normal stresses that any low frequency power transformer $(50 \mathrm{~Hz})$ can stand for:

- Reflection of voltage pulses at the impedance mismatching terminals

- Resonance inside the converter

- Uneven distribution of voltage pulses along the windings

The following parameters are to be considered to ensure a reliable application of any insulation material for any MFT:

- Voltage magnitude

- Voltage frequency (or pulse repetition rate)

- Partial discharge

- Pulse rise time

- Pulse duty cycle and polarity

\subsubsection{Effect of voltage amplitude}

Because of transient over voltages or temporary overloads, the voltage on or temperature of an insulation part used in an MFT placed inside a DAB converter can be much higher than the calculated stresses values at steady state operation. The safety margins for the withstand strength at the rated values are presented in the materials' data sheets. Because of the accelerated aging, when the voltage magnitude increases, the lifetime of the insulation system decreases. Many investigations have concentrated on the effect of voltage magnitude to the acceleration of the insulation material aging. The most reliable relation has been presented in IEC 61251 [91] as

$$
L=c E^{-n}
$$

where, $L$ is the time to breakdown, $E$ is the electrical field and $c$ and $n$ are the parameters depending on temperature and other environmental effects. Investigation made by Stone et al. in 1992 demonstrates how an increase in the applied voltage magnitude decreases the time to failure of an epoxy insulation [92].

\subsubsection{Effect of Frequency}

In general, like for mechanical fatigue, regardless of frequency, the same number of voltage impulses or cycles in a period causes the same degradation of an insulation material. This is shown by Johnston in 1979, during an extensive literature review where he has presented a table of summery 
for most of the efforts that has focused to the frequency acceleration of the voltage endurance [93]. However, he has also referred to the works which reported a nonlinear acceleration result. This inconsistency can be because of humidity or a change of material temperature resulting from dielectric losses in a long-term test. According to Johnston, a linearity factor can be gained for every specific experimental setup and it is shown that

$$
f_{1} t_{1}=k\left(f_{2} t_{2}\right)
$$

where $k$ is one, for most of the cases, if the temperature, humidity or test set-up effects are ignored.

\subsubsection{Effect of partial discharge}

Partial discharge is one of the physical processes which cause aging of insulation material. Chemical reactions, bombardment of cavity surfaces by charge carriers and local electrical field enhancement resulting from the discharge by-products, contribute to the creation of large holes and starting treeing activities [33].

Partial discharge inception voltage is the applied voltage when the electric field over an insulation material or system is higher than the required value for starting a PD activity. When an alternating voltage is applied, PD pulse trains can be repeated for each period (see Figure 2-19). Compared with PD pulses that appears stochastically, discharges which occurs at the voltages higher than the Repetitive Partial Discharge Inception Voltage (RPDIV) [94] are the main cause of the aging under square wave voltage regime.

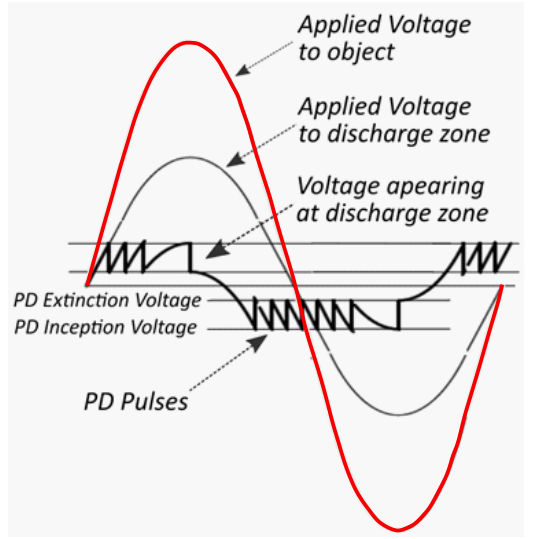

Figure 2-19. PD pulse train for each cycle of applied voltage [65]

\subsubsection{AC standard breakdown test methods}

To characterise the insulation materials, the main types of insulation tests are Insulation withstand or Breakdown (BD) test and Insulation aging test. The number of samples to undergo identical tests should be as high as possible to guarantee a reliable and repeatable result.

\section{For insulation oil}

IEC 60156 [95] describes a test method for the determination of AC breakdown voltage of transformer oil. Figure 2-20 demonstrates the electrode configuration for acquiring the insulation strength of transformer oil according to this standard.

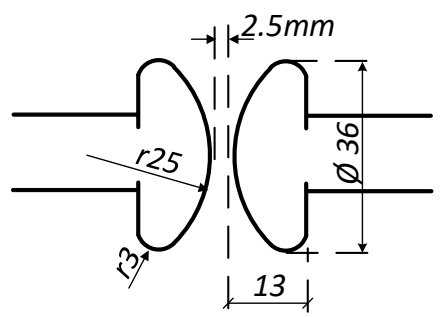

Figure 2-20 Standard electrode configuration for testing insulation strength of transformer oil [95] 


\section{For insulated wires}

The tests samples are prepared according to a well-defined standard method of IEC 60851-5 [96]. For each sample, two wire strands are twisted several times. To secure a defined contact area for similar tests, a load is applied during twisting the pairs (see Figure 3-26). The rate of voltage increase shall be defined (see Figure 2-21).

\section{For insulation tapes, plates or films}

Regarding high voltage testing of insulation materials, a series of articles and standards exists. To achieve more reliable results in a shorter period, the standardized methods are preferable to the experiments that are presented in the scientific articles. The following is list of standards which can be used:

- IEC 60243-1:2013, Test methods to determine the electric strength of insulating materials at power frequencies $(50-60 \mathrm{~Hz})$

- ASTM D149:2004, Test methods for electrical insulation tests of solid insulating materials at power frequencies

- IEC 60505:2011, Evaluation and qualification of electrical insulation systems

- IEC 61251:2015, Electrical insulating materials and systems AC voltage endurance evaluation

- IEC 62539:2007, Guide for the statistical analysis of electrical insulation breakdown data

\subsubsection{Testing combined insulations}

Testing withstand ability of single type materials may not be a real representation of withstand strength of a combined solid insulation system. At the interface of two solid insulation materials, the breakdown strength is governed by size and pressure of the voids filled of different insulating material (in case of testing in air is according to Paschen curve [97]). This is also true for the interface of insulation and electrodes.

\subsubsection{Mechanical electrode preparations}

Here are some notes presented in IEC 60243-1:2013 [98] regarding a test set-up that can guarantee a reliable and repeatable series of the tests:

- The electrodes should be kept clean, smooth and free from defects after each ACBD test.

- For most of the materials, increasing the thickness of the test object will result in lower electric strength and the results of the tests for different thicknesses are not directly comparable. The thickness of the specimen, time of voltage application, duration and intensity of surface discharges directly before a breakdown, affects the electric strength of most of the types of insulation materials [Ref IEC 60243-1:2013].

- The leads to the high voltage electrodes shall not affect the electrode setup in any way, nor change the electric field near the breakdown position.

\subsubsection{Handling of surrounding medium oil}

When oil is used as a surrounding medium, any discharge will pollute the oil and should therefore be avoided. Practical tests show that this is not easily achievable especially if the discharge current amplitude and duration cannot be kept short enough.

\subsubsection{Measuring voltage}

It is well accepted in the high voltage industry that it is the peak value of the applied voltage which shall be adjusted during standard withstand tests. For all the frequencies, the same peak value is applied. The Partial Discharge (PD) is monitored. 


\subsubsection{Rate of rise of voltage}

As the time of voltage application increases, the electric strength of most of the materials decreases with increasing the voltage application time [98]. Therefore, the rate of rise of AC voltage is important. One of the methods can be chosen; the rapid rise, the $20 \mathrm{~s}$ step-by-step or the slow rateof-rise (120 s - $240 \mathrm{~s}$ ) method.

According to ASTM D149-97a [99], the "Slow rate-of-rise test" is simpler and the preferable method to the "Short-time test" and "step-by-step test" method and gives more meaningful results when different materials (artificially contaminated in this case) are compared with each other. The test method is described in sub-clause 10-3, IEC 60243-1 [98].

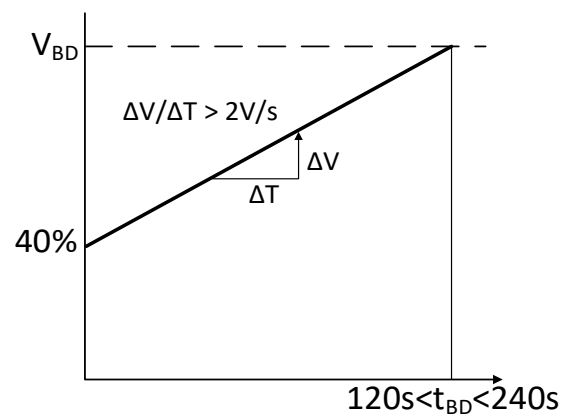

Figure 2-21. Voltage profile with a Slow rate-of-rise test according to IEC 60243-1

\subsubsection{Monitoring PD during an aging test or before an ACBD test Necessity of PD monitoring}

The partial discharge (PD) measurement is an accepted test for qualification of insulation systems. During insulation test of materials or a complete transformer, recorded PD signals include useful information about any possible design, or manufacturing mistake or a sign of undesirable aging.

\section{Proper PD measuring under square wave}

When it comes to a square wave signal on the terminals of an MFT, the transformer insulation will be under repetitive impulses of high $\mathrm{dv} / \mathrm{dt}$ stress. During the application of a square wave shape voltage to an MFT, as the PD signals become similar to the supply voltage signal, then it is very important to use a correct method to measure true PD signals.

\section{Measured PD during a dielectric test}

The existence of partial discharge should be controlled for a reliable and repeatable aging test. When increasing the applied voltage, the partial discharge (PD) starts at the PD Inception Voltage (PDIV). The PDIV depends on many parameters where just a part of them are originating from the nature of a material under test. The remaining parameters such as voids between the material and electrodes, discharges at the edges of electrodes or the insulation material surface where the touching surfaces ends, have a parasitic nature during a high voltage insulation test. 


\section{Characterisation of insulation material for HVDC MFT application}

The most important part of the insulation material specification data to be used for the DC insulation design of a HVDC MFT is the magnitude of its electrical conductivity, and as explained in section 1.2.3 a conductivity magnitude is not a straight forward predetermined value which can be find in any data base. Therefore, a great effort in this work is devoted to a series of conductivity measurement activities on mineral and ester transformer oils.

The other aspect that should be considered regarding characterisation of the insulation material used for production of a medium frequency transformer which is subjected to high frequency pulses from fast rising square wave voltage signals (see Figure 2-1a) is their voltage withstand strength under high frequency stress. This aspect is covered in section 2.5 and guidelines to tackle this challenge is presented.

\subsection{DC conductivity measurement setup}

High voltage DC conductivity measurements are performed using the background information presented in section 2.3. A complete test setup is developed which is demonstrated in Figure 3-1 and described in the following sections.

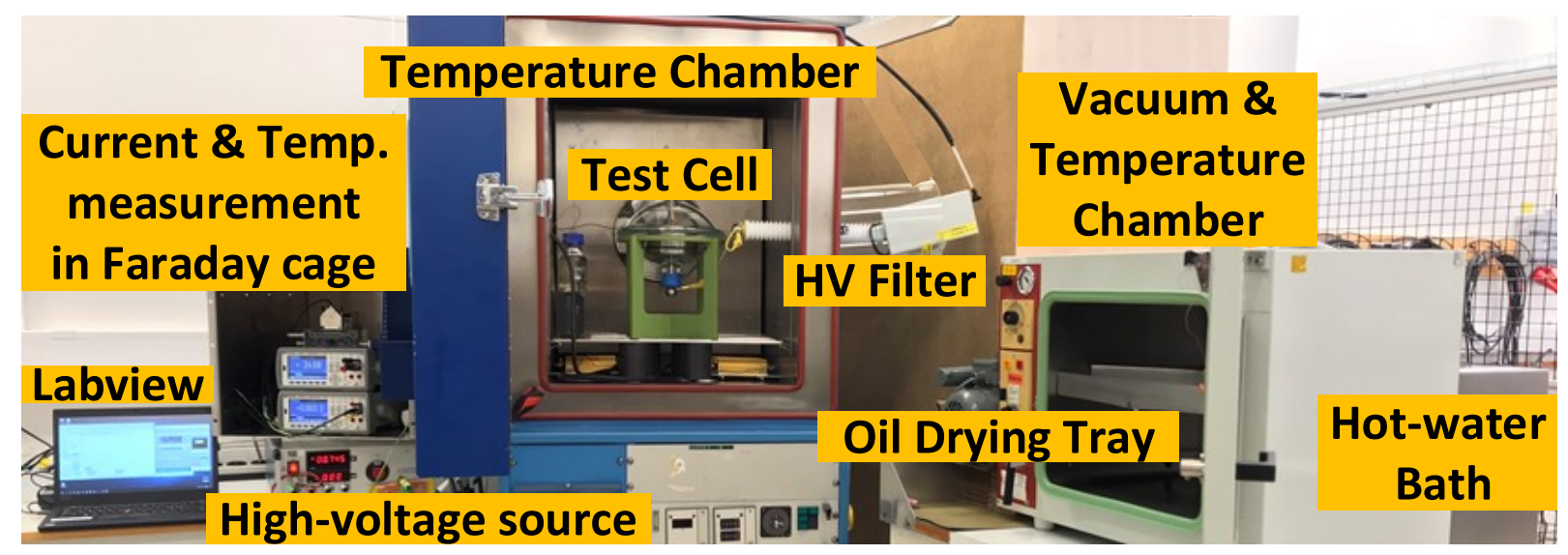

Figure 3-1. The test setup for conductivity measurements

Test cell is prepared according to precautions presented in section 2.3.2. In case of oil tests, a gap distance of $2 \mathrm{~mm}$ was held using dried and oil impregnated pressboard during the oil test. Stainless steel (EN 10020) having a maximum contact surface roughness of $4 \mu \mathrm{m}$ is used. In case of solid dielectrics, a sample thickness of $1 \mathrm{~mm}$ was used in the set-up. A minimum pressure of 10-20 $\mathrm{kPa}$ (1$\left.2 \mathrm{~N} / \mathrm{cm}^{2}\right)[52]$ is held using epoxy coated round lead metal sheets. 

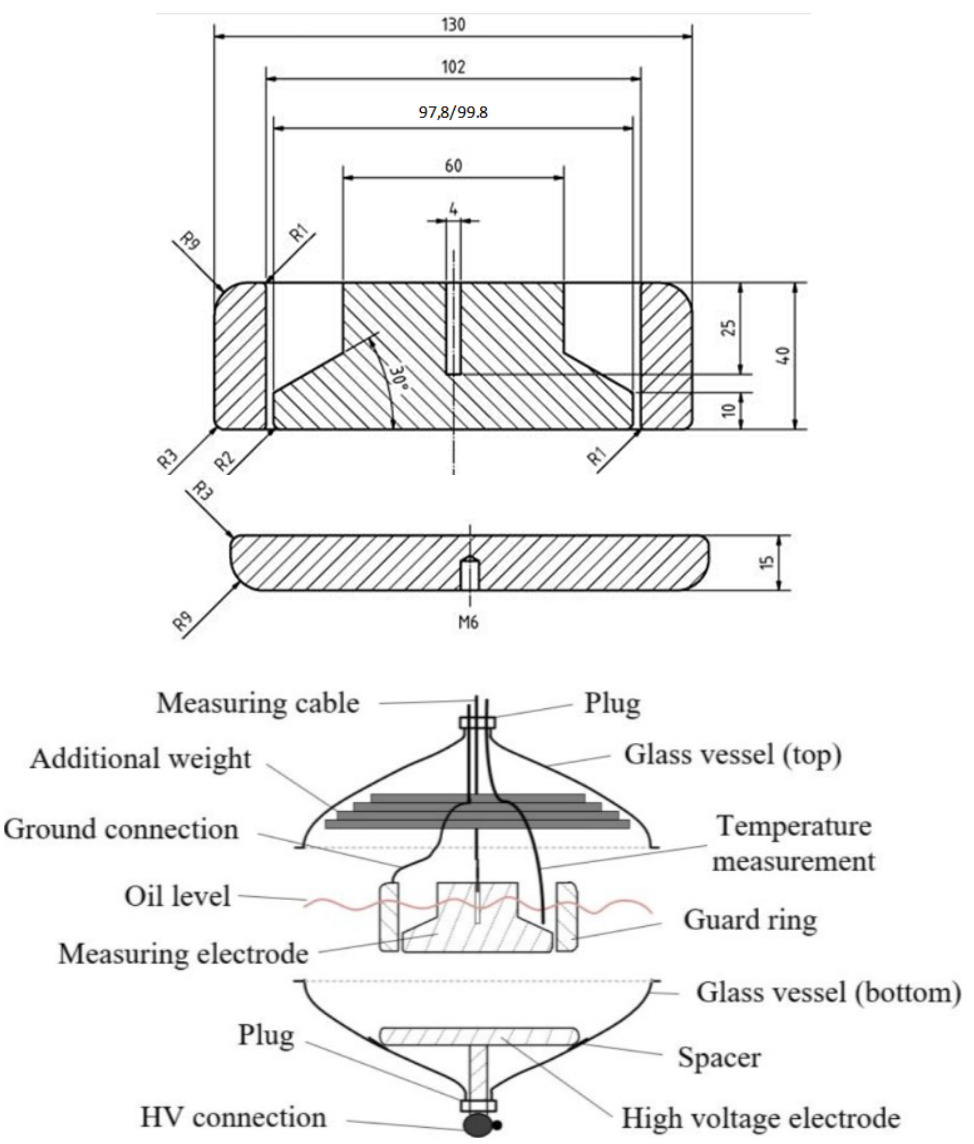
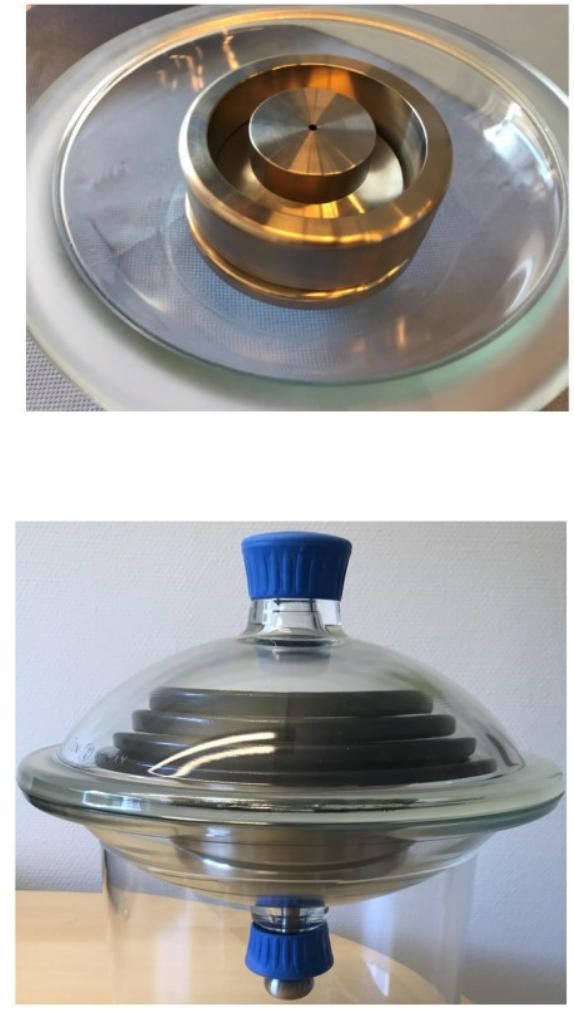

Figure 3-2. Electrode setup for high stress conductivity measurements [52]

The setup is placed in a temperature chamber. Since the measuring system is very sensitive to the external electrical and mechanical noises, to stabilise the temperature of the chamber during the polarisation phase, a set of series-parallel connected high power resistors is used as a heating setup to keep a constant temperature of the chamber during the whole period of measurement (see Figure 3-3) .

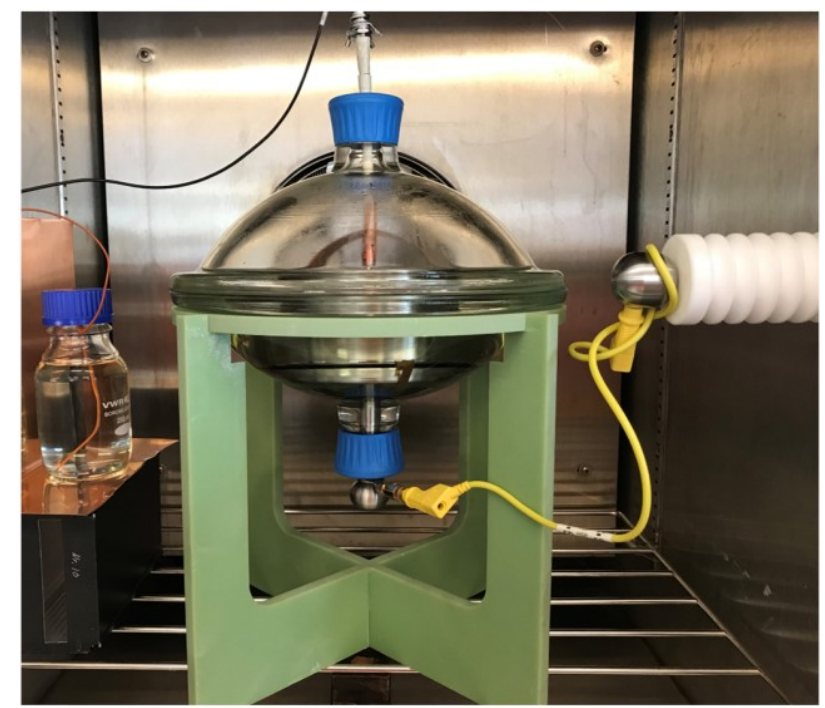

(a)

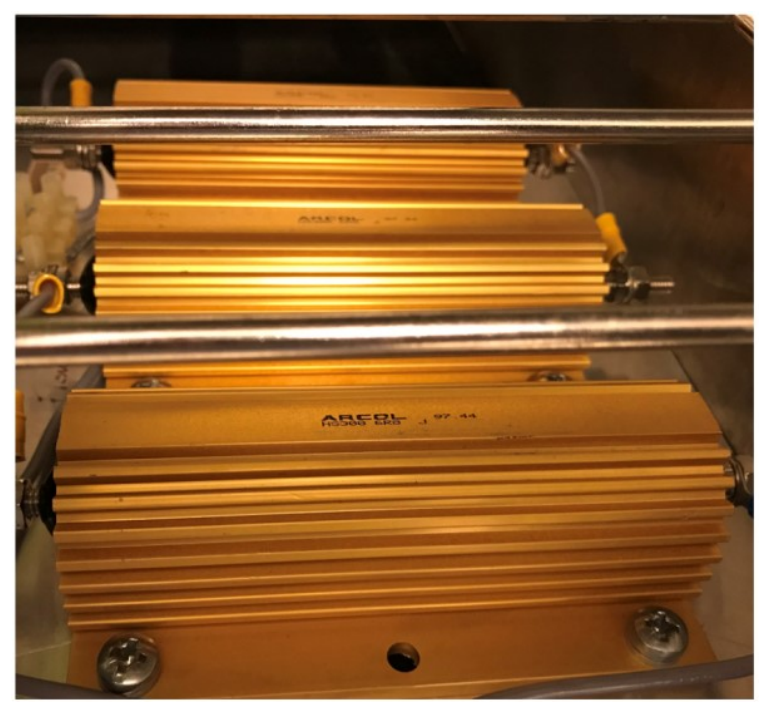

(b)

Figure 3-3. Measurement vessel inside a temperature-controlled chamber connected to HV DC bushing (a) and temperature stabilising heating resistors (b) 


\subsubsection{Current measuring and data acquisition system and its protection}

The current measuring circuit is as Figure 3-4 and similar to IEC 93, 1980 adopted for volume resistivity measurement.

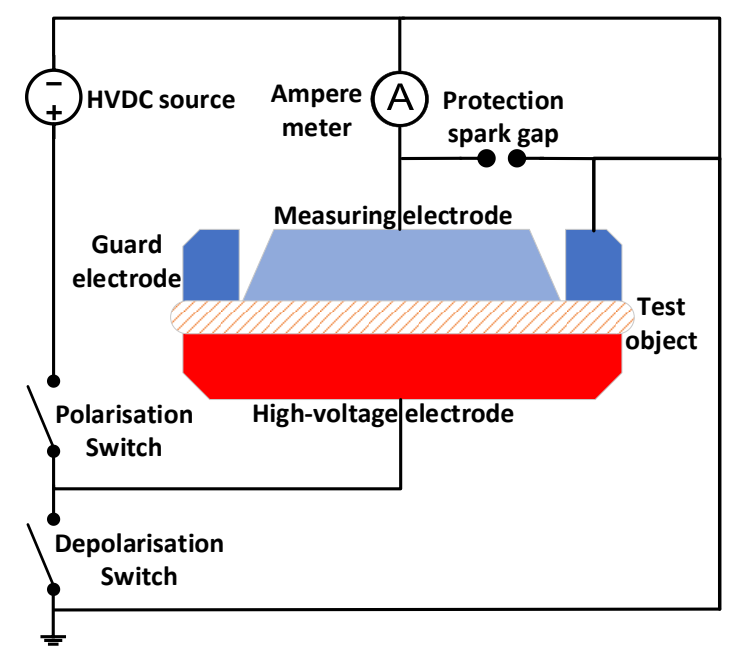

Figure 3-4. Conductivity measuring circuit diagram

The current measuring system consists of a Femto DLPCA-200 current amplifier having a current measuring range from $10 \mathrm{pA}$ to $10 \mathrm{~mA}$ (see Figure 3-5) and a Keysight 34470A Digital voltmeter.

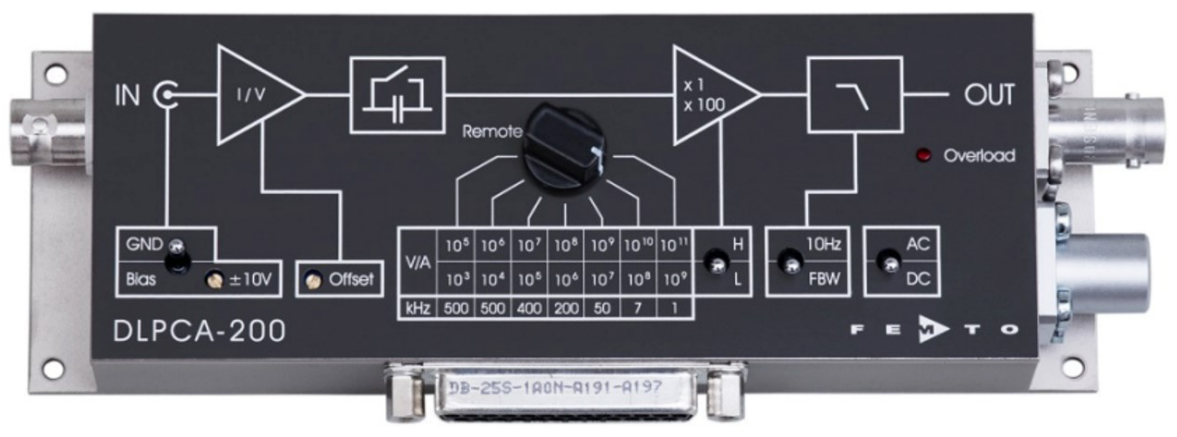

Figure 3-5. FEMTO DLPCA-200 current amplifier

A Labview based control and data acquisition software is developed for automatic amplifier gain setting as well as recording of the measured data. All the test condition data have been entered via the interface before starting each measurement, and the measurement data file is automatically named and saved every minute.

The current measuring system should be protected against high applied voltage and high short circuit current. Protection against an input current higher than the maximum permissible $10 \mathrm{~mA}$ of the Femto DLPCA-200 current amplifier, can usually be fixed using a high voltage high ohmic series resistance in the circuit. In this case it is done by setting the current limit of the high voltage DC power supply to a current lower than $10 \mathrm{~mA}$ and therefore no external current limiting series resistance is needed.

Protection against a possible high voltage transient has been done by a parallel connection of the following elements:

- A 2049-07 medium duty 2 electrode gas discharge tube having a 75V DC sparkover@100V/s

- Two parallel reverse coupled 1N3595 small signal diodes with maximum reverse current of 1nA@150 V and a minimum forward voltage of $0.5 \mathrm{~V}$ 
- A TransGuard multilayer ceramic transient voltage suppressor having minimum possible leakage current at the working voltage and a working voltage higher than the voltage at the input of Femto DLPCA-200 which is maximum $10 \mathrm{uAx} 60 \Omega=0.6 \mathrm{mV}$. In this case a VC060314A300 is used.

The manufactured high voltage protection module is subjected the maximum predicted input voltage of the current amplifier and the leakage current is measured using a precision source meter (see Figure 3-6). The measured leakage current at $0.6 \mathrm{mV}$ on the input, is less than $0.5 \mathrm{pA}$ which is much less than the typical measured conductivity current of nearly 100 pA.

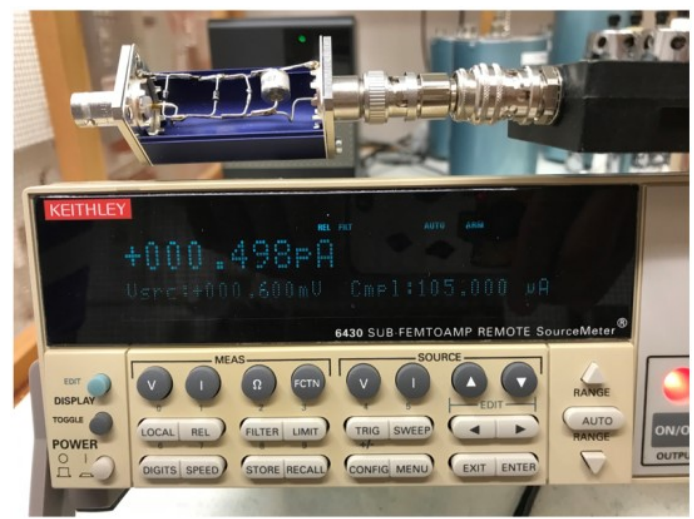

Figure 3-6. Test setup for verification of overvoltage protection system

Figure 3-7 demonstrates the current amplifier, voltage measuring device, test cell temperature meter, and a high voltage DC source with polarity reversing function.

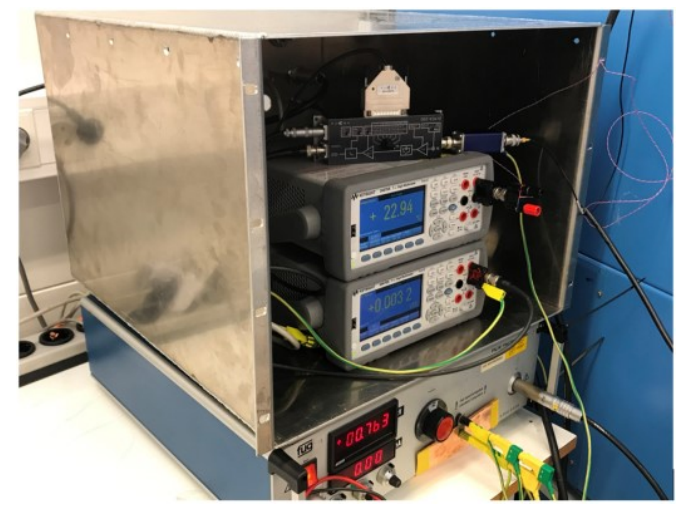

Figure 3-7. Conductivity measurement setup inside a noise suppression cage

\subsubsection{High voltage application system and safety}

A Fug HCN 140M 0-35000 V High voltage DC power supply equipped with an adjustable current limiting system of 0-4 mA is used a source. A low inductance ground connection has been prepared at the body of the high voltage DC source.

\subsubsection{Mitigation of noises}

A high voltage filter consisting of series resistors and parallel capacitors is connected to the delivery point of high voltage $D C$ to the chamber. The resistance is composed of ten $22 \mathrm{M} \Omega$ in series and the capacitance is combined of 6 series and 4 parallel capacitors. The selected parts were double metallized film pulse capacitors with a polypropylene dielectric PHE450 47nF, $2000 \mathrm{~V}$ and Metal Glaze Film Resistors MGRF $22 \mathrm{M} \Omega, 2 \mathrm{~W}, 3500 \mathrm{~V}$. Effort has been taken to avoid discharges because of low isolation distances and sharp high voltage conductors (see Figure 3-8). 


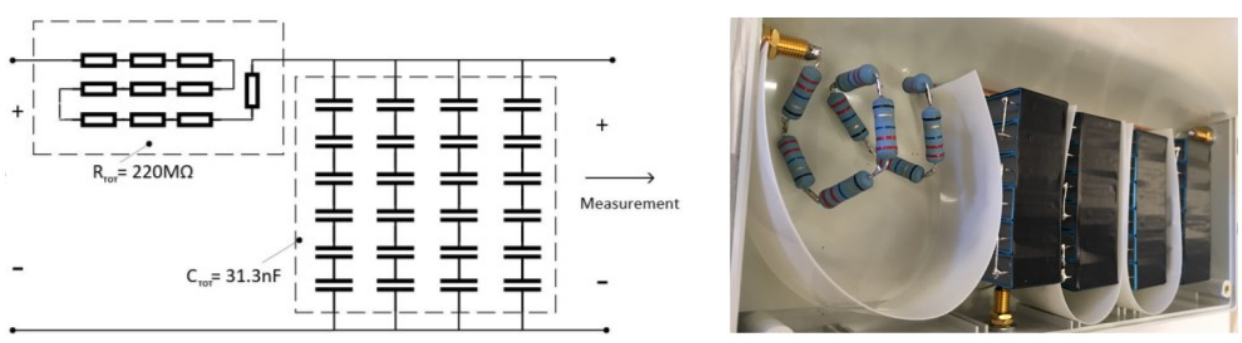

Figure 3-8. Designed and manufactured HV filter

The complete measuring setup is placed inside a grounded metal shielding box. The mains supply has been done using a 1:1 isolating transformer. The multi-meters and the current amplifier have been connected by a fibreoptic hub and cables to the computer.

\subsubsection{Preparation and testing of samples}

Oil samples and the oil in the container for testing pressboard samples were dried (down to $5 \mathrm{ppm}$ ) according to the method explained in reference [68]. A setup including a hot water bath (see Figure 3-9a), a heating and vacuum chamber and a stainless-steel tray were used for this purpose (see Figure $3-9 b$ ). The process, simply explained, is to warm up the oil to $80^{\circ} \mathrm{C}$, feed it into a vacuum chamber through a Swagelok severe-service union-bonnet needle valve, which is already warmed up to $80^{\circ} \mathrm{C}$, spread a thin layer of oil on the steel tray under a vacuum less than $1 \mathrm{mbar}$, collect the dried oil in the lower part of measuring vessel, turn off the heating and let the system cool down to room temperature. The vacuum level was constant during the whole process. This process takes minimum 72 hours.

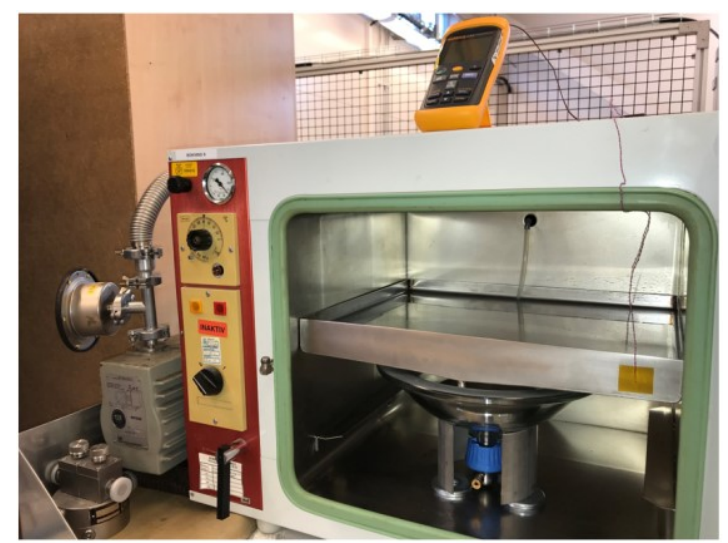

(a)

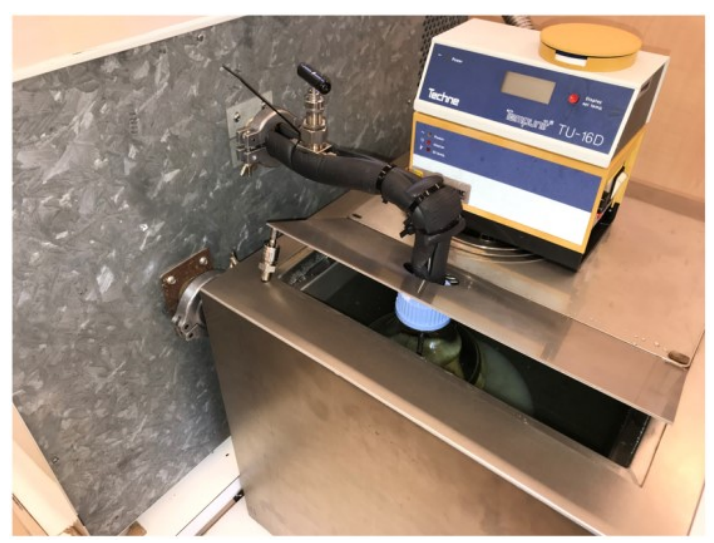

(b)

Figure 3-9. Dry oil preparation setup; $\boldsymbol{A}$ heating and vacuum chamber equipped inside with a steel tray for drying and collecting the dried oil (a), Regulated hot water bath feeding the hot oil through a needle valve into a heating and vacuum chamber (b)

The mineral oil vapor-pressure curve [100] (see Figure 3-10a) was used to adjust the temperature and vacuum level of the chamber for the most effective drying process of the samples [7]. For example, applying $10 \mathrm{mbar}$ and $60^{\circ} \mathrm{C}$ will evaporate the water from a humid mineral transformer oil without evaporating the oil itself.

A Vaisala MM70 oil humidity meter was used to measure the humidity content of the oil samples (see Figure 3-10b). The humidity content of the mineral oil before drying, before starting the conductivity tests and at the end of measurements were registered. The humidity content of the oil was checked after the processing and it was found to be less than $5 \mathrm{ppm}$ for mineral oil and less than $30 \mathrm{ppm}$ for ester oil before the conductivity measurement tests were done. 
The preparation of solid samples was done according to section 20.3 of IEC60641-2. 2004 [101]. In this work, first the new and clean samples are placed with a $1 \mathrm{~mm}$ distance in the steel tray in the chamber. Later the temperature is increased to $80{ }^{\circ} \mathrm{C}$ and after stabilising the temperature, the objects is set under full vacuum for the next 72 hours. Finally, the same procedure as the one for the oil sample preparation was followed to impregnate the hot oil in the already dried cellulose solid insulations.

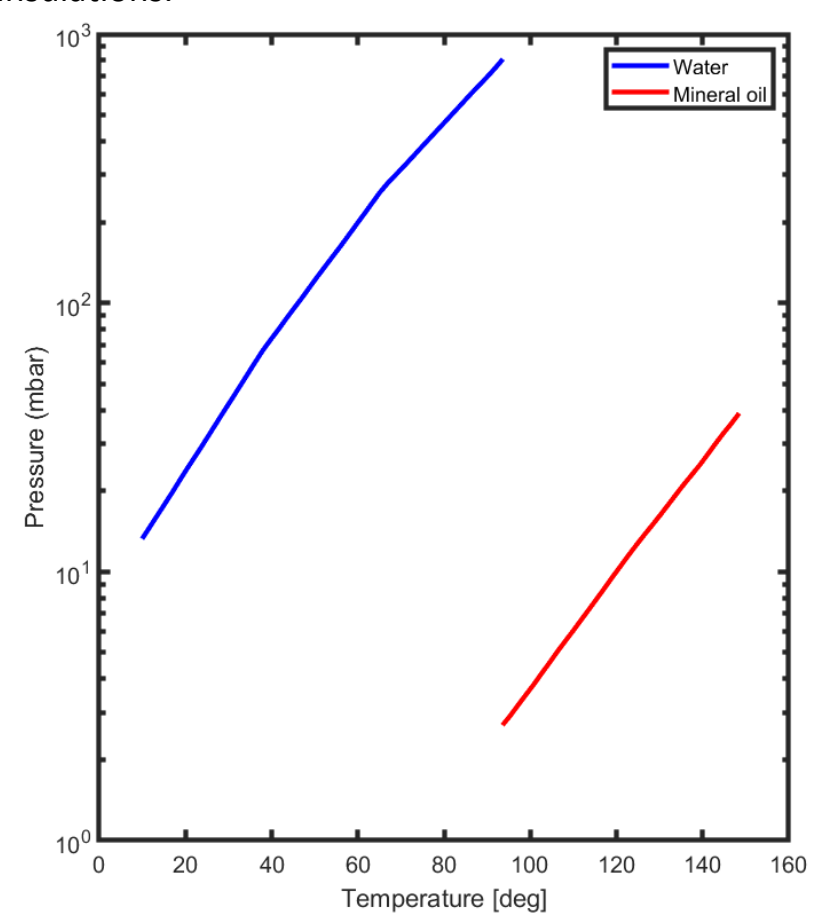

(a)

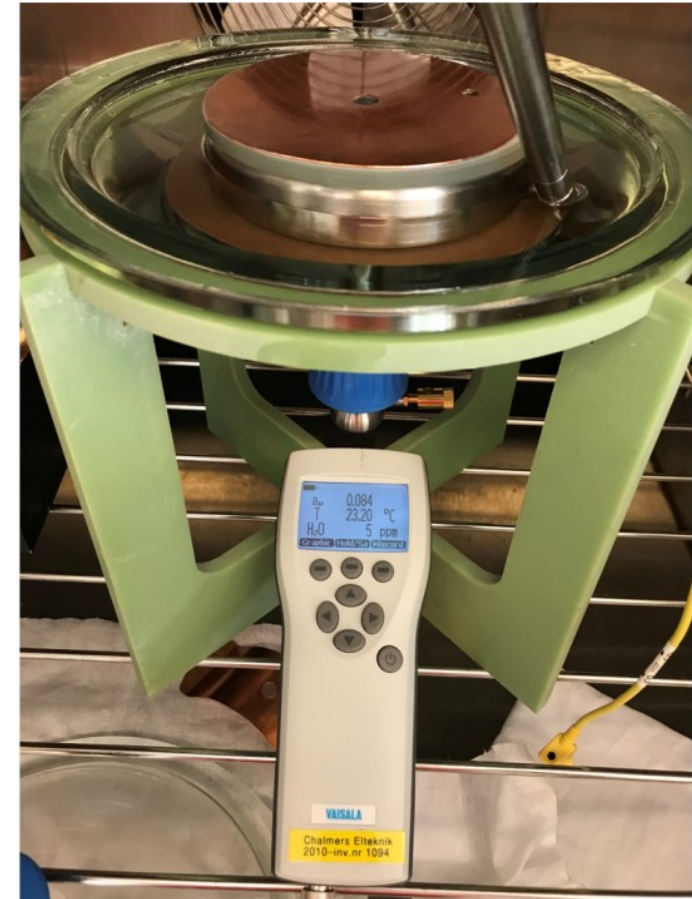

(b)

Figure 3-10. The vapor-pressure curve used and the humidity measuring equipment; The vapor-pressure curves to adjust temperature and vacuum during drying process (a), Vaisala meter showing the oil water-content after treatment (b)

\subsection{Conductivity measurement procedure}

Each experiment was conducted by applying a high DC test voltage and recording the time variation of the current through the test object until it reached a steady state (polarization phase). Directly after, the test object was short-circuited for a time interval of at least twice of the polarization time and the current was recorded again (depolarization phase). The process of a Polarisation, Depolarisation Current (PDC) measurement in demonstrated in Figure 2-16.

The conductivities were calculated in the range of the applied electric field of (1-6) kV/mm and (1-12) $\mathrm{kV} / \mathrm{mm}$ for oil and OIP, respectively, and for a temperature range of $(30-90){ }^{\circ} \mathrm{C}$.

\subsection{Conductivity measurement results}

In this section, OIP refers to the cellulosic transformer board impregnated with the mineral or ester transformer oil. The tested mineral transformer oil is Nynas Nytro $10 \mathrm{XN}$ and the ester transformer oil is Midel 7131 synthetic ester. The electrical permittivity values of mineral and ester oil and OIP are $2.2 \& 4.4$ and $3.5 \& 4.6$ respectively.

\subsubsection{Mineral oil and OIP}

Although in case of mineral oil, the conductivity data for high voltage DC applications are already given in literature $[52,76,102,103]$, the measurements have been done to support a reliable design of the measuring system. 
Oil and OIP samples considered for the manufacturing of a prototype MFT were tested and the results are presented in Figure 3-11 and Figure 3-12 respectively.

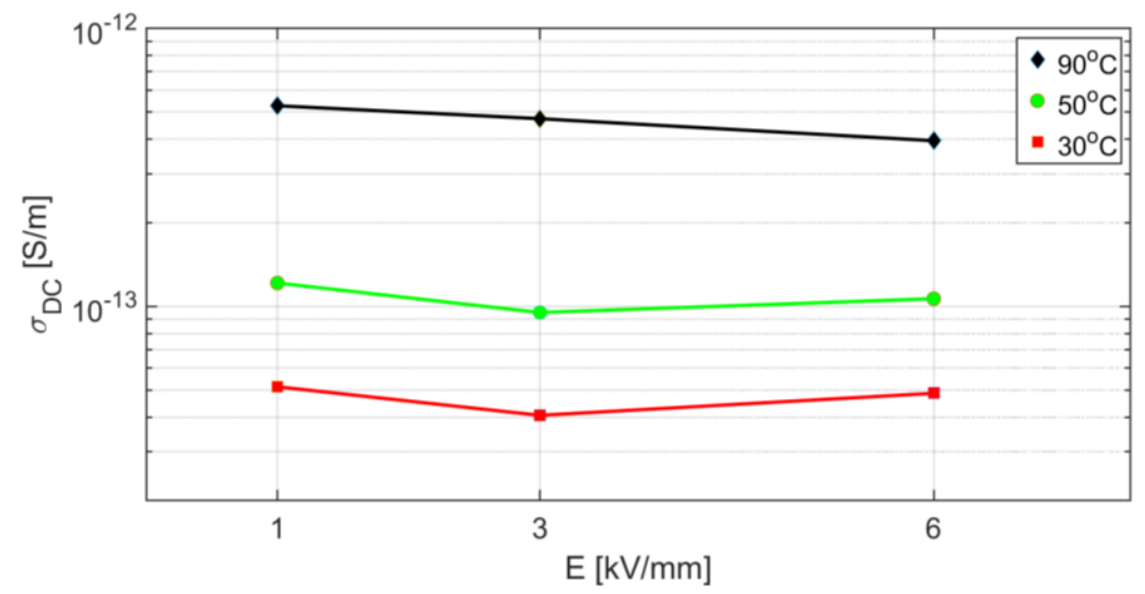

Figure 3-11. Conductivity measurement results of mineral oil

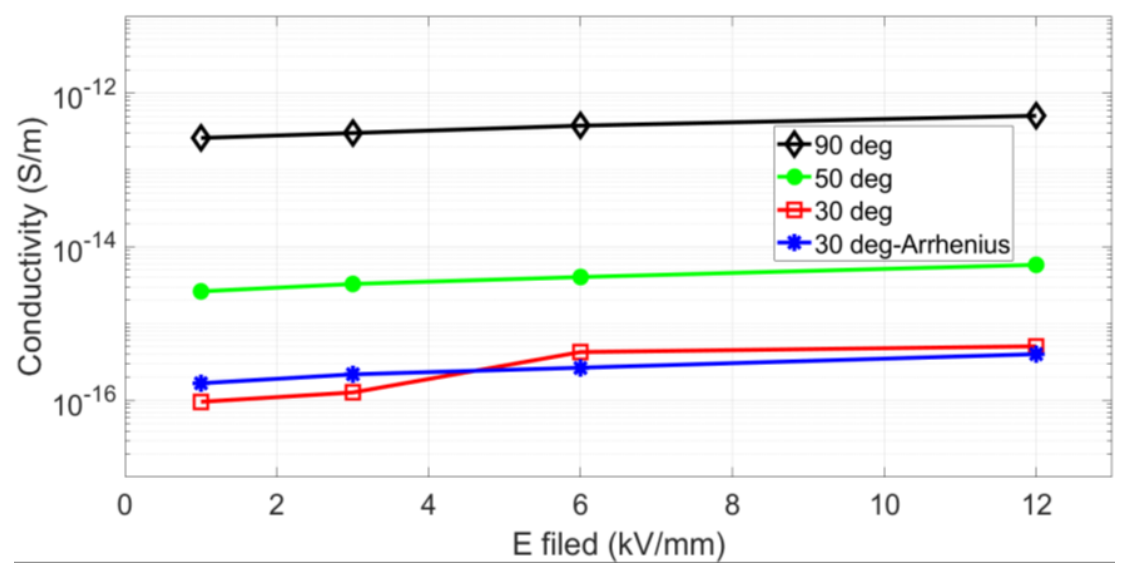

Figure 3-12. Conductivity measurement results of OIP

The measured conductivity values, which are also summarized in Table $3-1$, strongly depend on temperature, and a weak dependency to the electric stress can be observed as well. This reflects the impact of the thermal and electric stresses on the electro-chemical processes in the oil and on oilpressboard interfaces, in particular charge injection and accumulation, ionic drift, recombination, etc. As explained in section 2.2.10 these dependencies have an impact on the field dynamics in the insulation system and thus on the design of the HVDC MFT. The stress distribution on a simple series of insulation layers depends on the Conductivity Ratio $(C R)$ of oil to OIP: $C R=\sigma_{\text {Oil }} / \sigma_{\text {OIP }}$. According to Table 3-1, the $C R$ values have a large scatter ranging from 294 at $30^{\circ} \mathrm{C}$ to 1 at $90^{\circ} \mathrm{C}$. The effect of $C R$ on the electric field stress on the different points of the transformer will be discussed in section 4.2.

Table 3-1. A summary of conductivity measurement results for mineral oil

\begin{tabular}{|c|c|c|c|c|c|}
\hline & & $1 \mathrm{kV} / \mathrm{mm}$ & $3 \mathrm{kV} / \mathrm{mm}$ & $6 \mathrm{kV} / \mathrm{mm}$ & $12 \mathrm{kV} / \mathrm{mm}$ \\
\hline \multirow{3}{*}{ Oil } & $30 \circ \mathrm{C}$ & $5 \times 10^{-14}$ & $3.9 \times 10^{-14}$ & $5.8 \times 10^{-14}$ & - \\
\hline & $50 \circ \mathrm{C}$ & $1.2 \times 10^{-13}$ & $9.5 \times 10^{-14}$ & $1.1 \times 10^{-13}$ & - \\
\hline & $90 \circ \mathrm{C}$ & $5.2 \times 10^{-13}$ & $4.6 \times 10^{-13}$ & $3.9 \times 10^{-13}$ & - \\
\hline \multirow{3}{*}{ OIP } & $\begin{array}{c}30^{\circ} \mathrm{C} \\
\text { (Arrhenius) }\end{array}$ & $1.7 \times 10^{-16}$ & $2.2 \times 10^{-16}$ & $2.7 \times 10^{-16}$ & $4 \times 10^{-16}$ \\
\hline & $50 \circ \mathrm{C}$ & $2.6 \times 10^{-15}$ & $3.3 \times 10^{-15}$ & $4 \times 10^{-15}$ & $5.8 \times 10^{-15}$ \\
\hline & $90 \circ \mathrm{C}$ & $2.6 \times 10^{-13}$ & $3 \times 10^{-13}$ & $3.8 \times 10^{-13}$ & $5.1 \times 10^{-13}$ \\
\hline \multirow{3}{*}{$C R$} & $30{ }^{\circ} \mathrm{C}$ & 294 & 177 & 215 & - \\
\hline & $50 \circ \mathrm{C}$ & 46 & 29 & 27 & - \\
\hline & $90 \circ \mathrm{C}$ & 2 & 1.5 & 1 & - \\
\hline
\end{tabular}


The results have a good correspondence with one of the most reliable results presented by Küchler [76].

\subsubsection{Ester oil and OIP}

After a complete set of conductivity measurements on mineral oil and OIP which provided a series of results which are comparable with the previous literature, the method is applied to characterize the biodegradable synthetic ester oil under HVDC stress.

As preliminary measurements showed an unstable and varying results for the polarisation current of ester oil, to get stable current curves it is decided that a conditioning is to be performed on the ester oil and the polarization time to be increased from $1 \mathrm{~h}$ (as was the case for mineral oil) to $3 \mathrm{~h}$ for the ester oil. To reach repeatable results it is also decided to perform a set of conditioning stress applications before each measurement [62]. In case of ester OIP it is performed like the measuring method for mineral OIP and a 3 hours measurement is performed. Figure 3-13 and Figure 3-14 demonstrate the test duration for ester oil and ester OIP respectively.

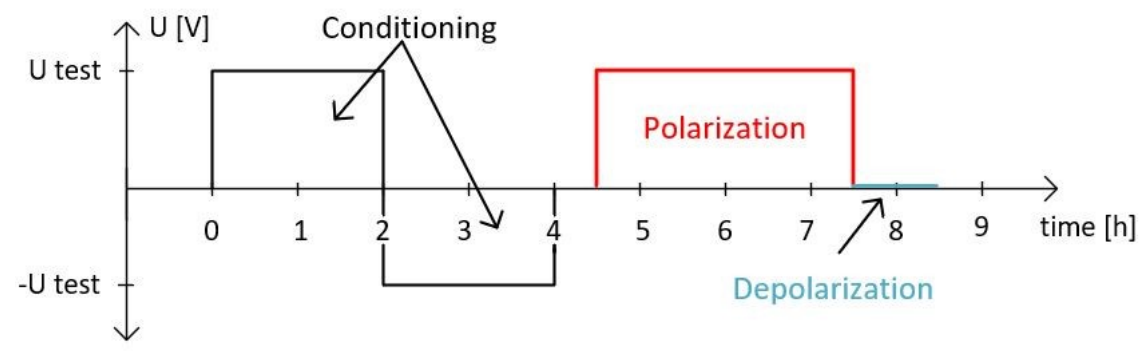

Figure 3-13. Measurement technique for ester oil

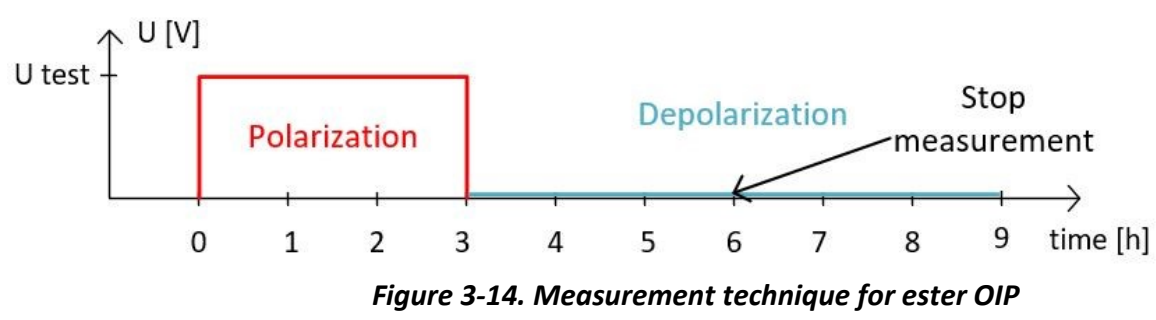

As it seemed that the depolarisation takes a longer time than expected, the measurements performed on ester OIP required a minimum of three hours depolarisation time at $90{ }^{\circ} \mathrm{C}$ (see Figure 3-15).

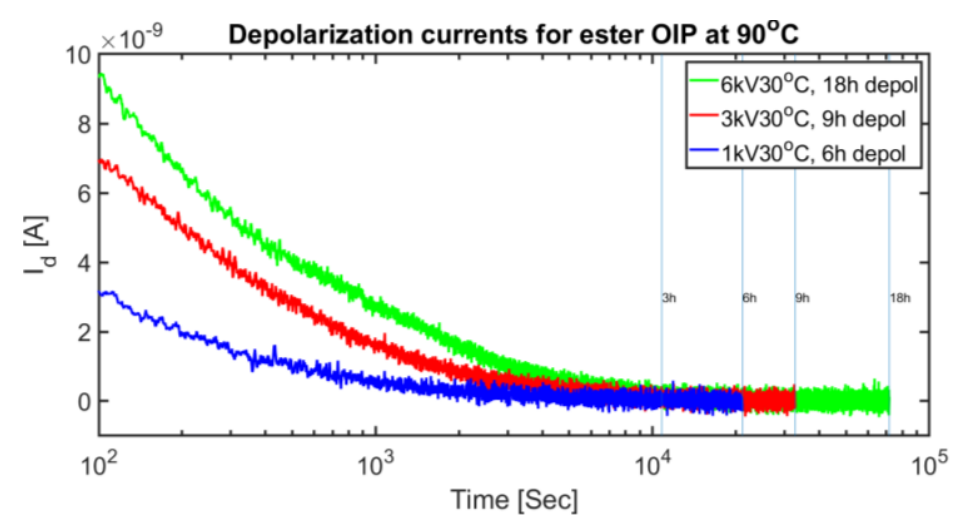

Figure 3-15. The minimum depolarisation time required at $90^{\circ} \mathrm{C}$

A summary of measurements done on the ester oil is presented in Figure 3-16. 


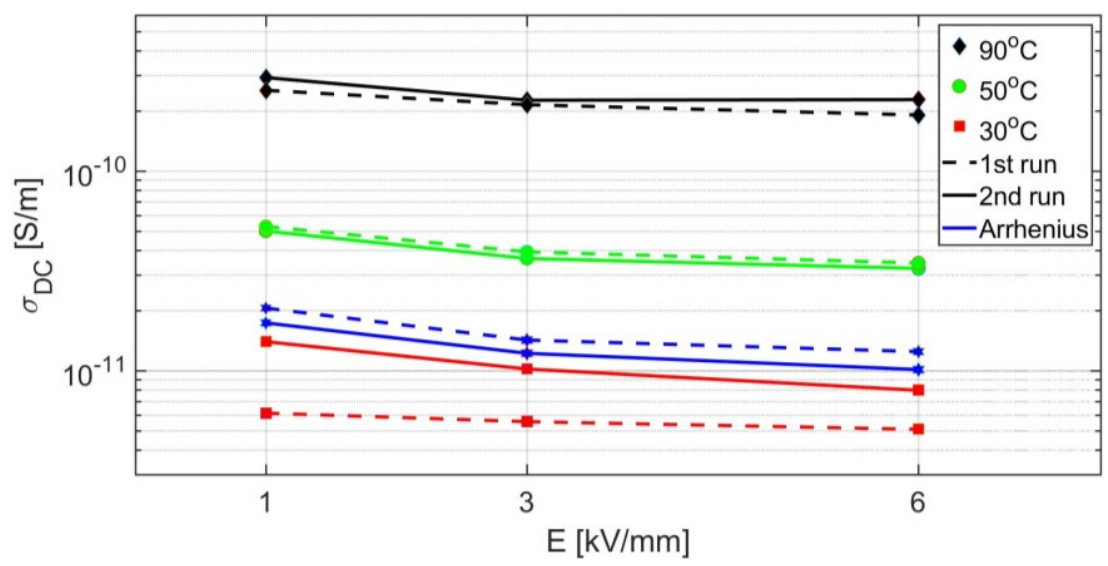

Figure 3-16. A summary of conductivity measurements of ester oil

The ester OIP measurements are presented in Figure 3-17.

For the both ester oil and OIP, the results show that, the conductivity values of the second run measured at $30{ }^{\circ} \mathrm{C}$ have good agreement with the Arrhenius calculations. For the first run, as the results of 50 and $90^{\circ} \mathrm{C}$ were available, the Arrhenius method can be applied to determine the values of conductivity at $30^{\circ} \mathrm{C}$. The measured values on the first $30^{\circ} \mathrm{C}$ run are not correct, and the results of the second run are reliable.

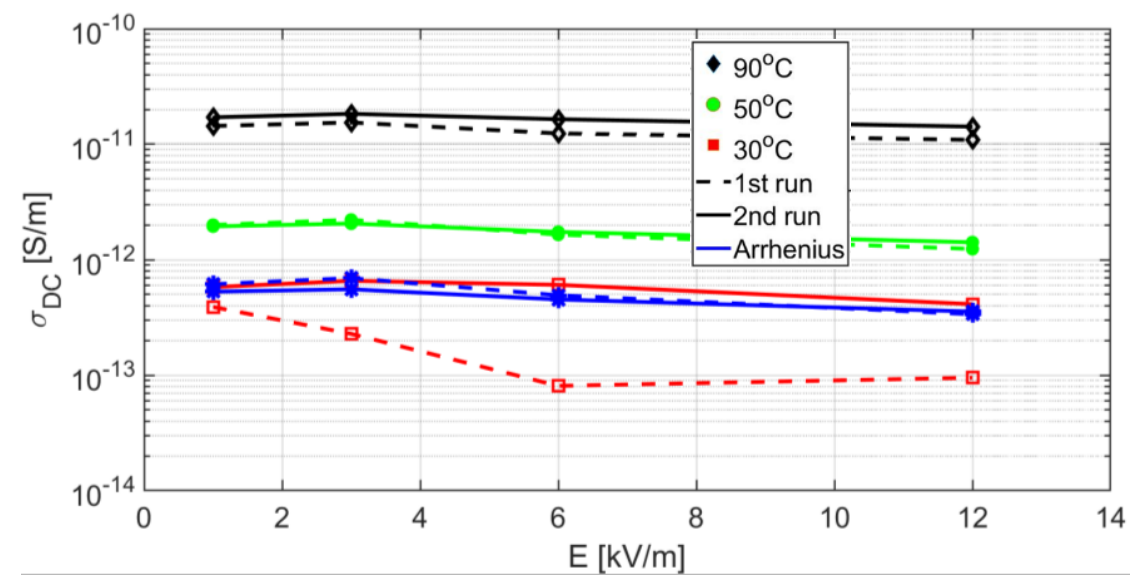

Figure 3-17. A summary of conductivity measurements of ester OIP

A summary of conductivity measurement results for ester oil and OIP is presented in Table 3-2. The results of the second series of ester OIP measurements together with the results of the first series of ester OIP measurements (with using Arrhenius results for $30^{\circ} \mathrm{C}$ ) can be preliminary used for the insulation design of an HVDC ester MFT prototype.

Table 3-2. A SUMMRY OF CONDUCTIVITY MEAUREMENT RESULTS for ester oil

\begin{tabular}{|c|c|c|c|c|c|}
\hline & & $1 \mathrm{kV} / \mathrm{mm}$ & $3 \mathrm{kV} / \mathrm{mm}$ & $6 \mathrm{kV} / \mathrm{mm}$ & $12 \mathrm{kV} / \mathrm{mm}$ \\
\hline \multirow{4}{*}{ Oil } & $30 \circ \mathrm{C}$ & $1.4 \times 10^{-11}$ & $1.0 \times 10^{-11}$ & $8.0 \times 10^{-12}$ & - \\
\cline { 2 - 6 } & $50{ }^{\circ} \mathrm{C}$ & $5.0 \times 10^{-11}$ & $3.6 \times 10^{-11}$ & $3.2 \times 10^{-11}$ & - \\
\cline { 2 - 6 } & $90{ }^{\circ} \mathrm{C}$ & $2.9 \times 10^{-10}$ & $2.3 \times 10^{-10}$ & $2.3 \times 10^{-10}$ & - \\
\hline \multirow{4}{*}{ OIP } & $30{ }^{\circ} \mathrm{C}$ \\
& (Arrhenius) & $6.1 \times 10^{-13}$ & $6.9 \times 10^{-13}$ & $4.9 \times 10^{-13}$ & $3.4 \times 10^{-13}$ \\
\cline { 2 - 6 } & $50 \circ \mathrm{C}$ & $2.0 \times 10^{-12}$ & $2.22 \times 10^{-12}$ & $1.6 \times 10^{-12}$ & $1.2 \times 10^{-12}$ \\
\cline { 2 - 6 } & $90 \circ \mathrm{C}$ & $1.4 \times 10^{-11}$ & $1.5 \times 10^{-11}$ & $1.2 \times 10^{-11}$ & $1.1 \times 10^{-11}$ \\
\hline \multirow{3}{*}{$\mathrm{CR}$} & $30 \circ \mathrm{C}$ & 23 & 15 & 16 & - \\
\cline { 2 - 6 } & $50 \circ \mathrm{C}$ & 25 & 16 & 20 & - \\
\cline { 2 - 6 } & $90 \circ \mathrm{C}$ & 20 & 15 & 18 & - \\
\hline
\end{tabular}


Limited information about the conductivity of biodegradable ester oil under HVDC stress is accessible $[61,104]$ and in case of ester OIP there is even less information available [62]. Figure 3-18 demonstrates comparisons between the measurement results of this work with the measurements presented in literature.

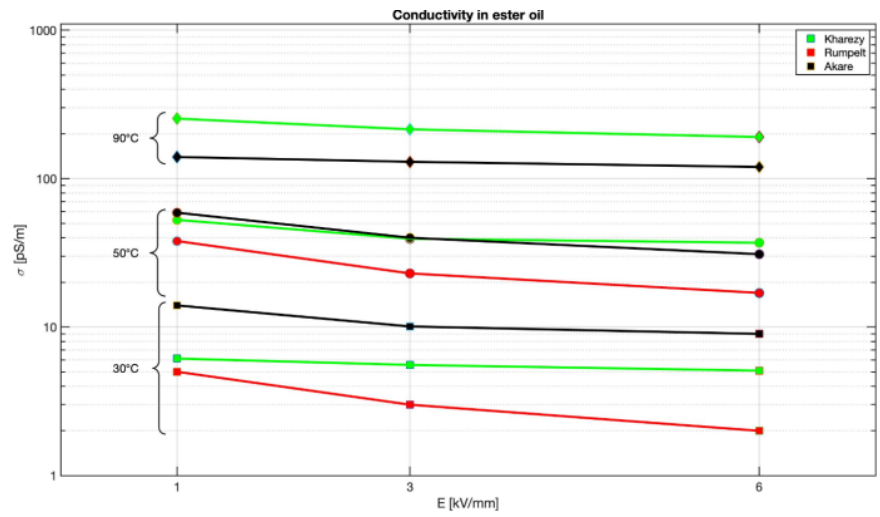

Figure 3-18. A comparison of measurement results with the conductivity values given in literature; green: Kharezy, red: Rumpelt [104] and black: Aakre [61])

\subsubsection{Mineral oil/OIP versus ester oil/OIP}

Figure 3-19 is provided for the purpose of comparison of the measurement results on the mineral and ester oil and OIP.
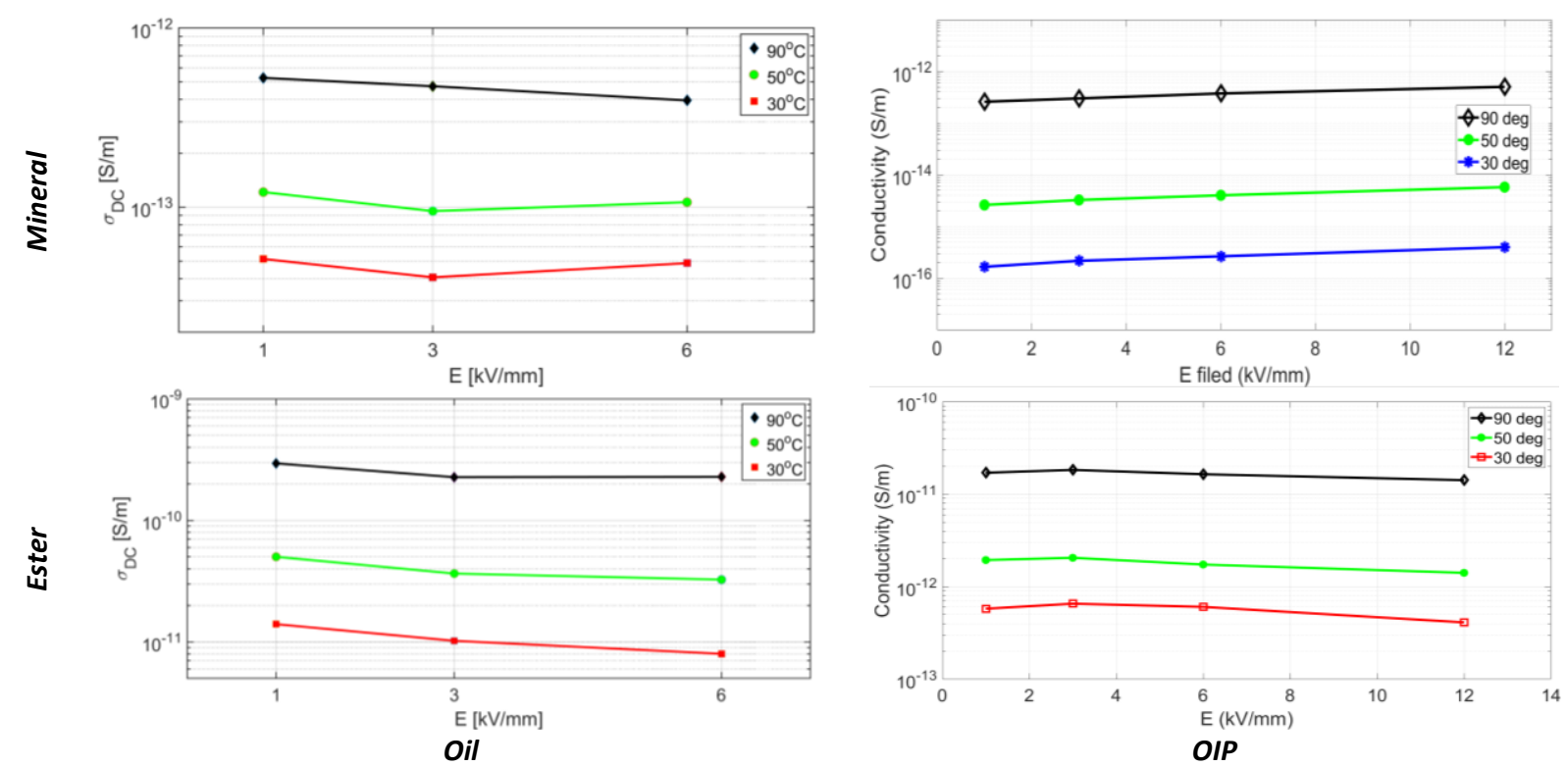

Figure 3-19. Comparison of the measurement results on the mineral and ester oil and OIP

The most important difference in the results is the higher values of conductivity for the ester oil. It is at $30{ }^{\circ} \mathrm{C}, 100-130$ times, at $50{ }^{\circ} \mathrm{C}, 320-430$ times and at $90{ }^{\circ} \mathrm{C}, 450-480$ times higher.

In mineral OIP the moisture increased from $5 \mathrm{ppm}$ to $16 \mathrm{ppm}$ and in ester OIP the increase level was from $46 \mathrm{ppm}$ to $225 \mathrm{ppm}$. It seems that the ester OIP can absorb more water without significantly increase the conductivity values.

A simple comparison of the conductivity values of ester and mineral oil and OIP highlights that the $C R$ values of ester oil/OIP are not as much temperature and voltage stress dependent as the mineral oil/OIP is. This reduces the dynamic stress level of the design in case of using ester oil and OIP. It is also noticeable that the ester oil/OIP conductivities are several hundred times higher than the mineral oil/OIP conductivities. This causes the time constants to be lower (see 4.2) and consequently 
results in a shorter time for the transformer to reach the steady state condition (see Figure 3-20b). The space charge accumulation time is much lower in the case of ester oil (see Figure 3-20d).

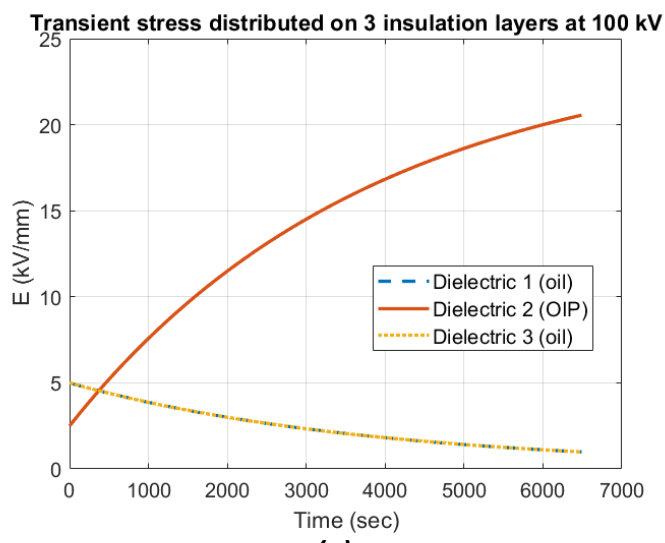

(a)

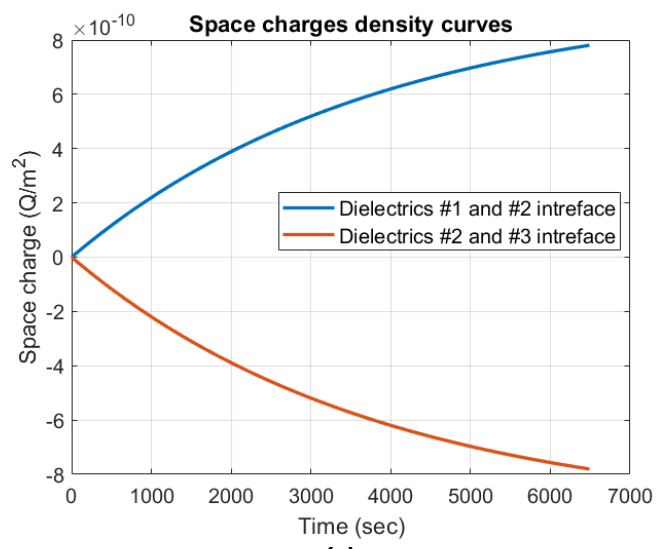

(c)

Mineral oil $\left(\sigma=5 \times 10^{-14}\right)$ and OIP $\left(\sigma=1.7 \times 10^{-16}\right)$

Figure 3-20. Space charge accumulation curve in a three-layer, oil-OIP-oil insulation system at $30^{\circ} \mathrm{C}$ and $1 \mathrm{kV} / \mathrm{mm}$

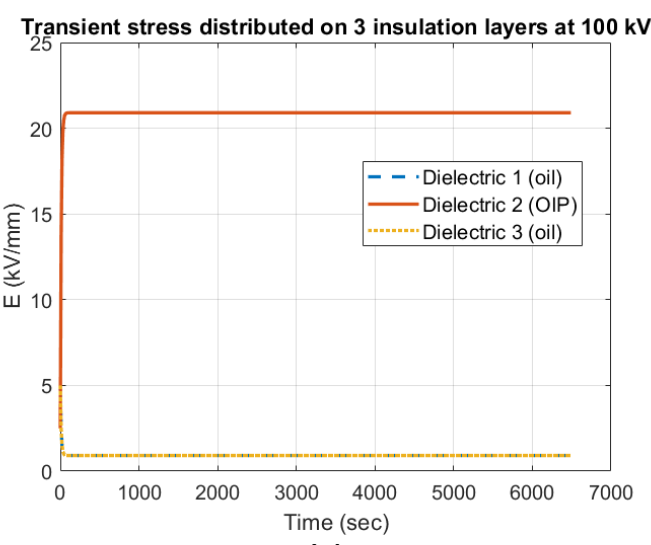

(b)

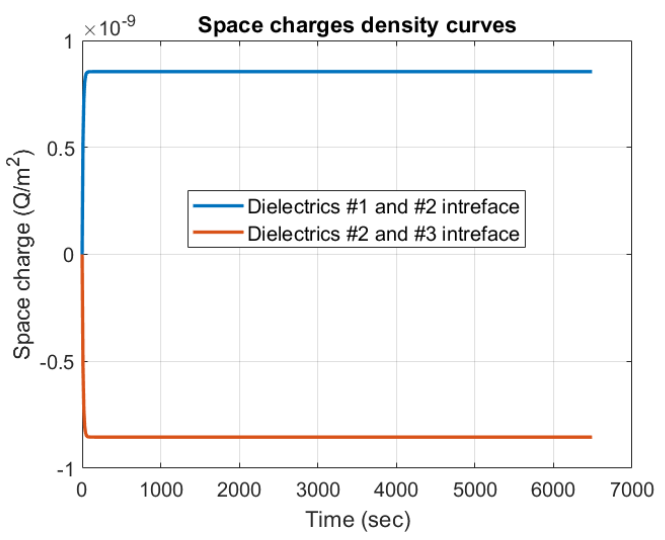

(d)

Ester oil $\left(\sigma=1.4 e^{-11}\right)$ and OIP $\left(\sigma=6.1 e^{-13}\right)$

\subsection{AC breakdown simulations and test conditions}

Based on the background information presented in section 2.5 the following activities were performed in this work.

\subsubsection{Effect of shape of electrodes and surrounding medium to the results of the tests}

Application of an electric field should not be done in such a way that any other phenomena disturb the main purpose of the voltage application. The intension is to simulate the actual electric field only inside the insulation as it will happen during a real application of the insulation material. Any discharge on the surface of the material can create heat and gas bubbles which in turn destroys the material and the electric field distribution inside it.

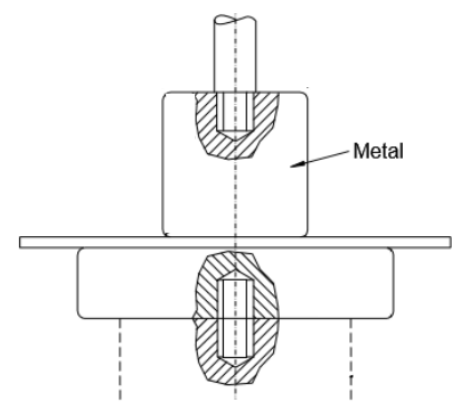

Figure 3-21. IEC 60243-1, proposed electrode setup [98] 
IEC 60243-1 has proposed the use of specially shaped electrodes for testing of electric strength of tapes (see Figure 3-21). Figure 3-22 demonstrates a FEM simulation of the IEC proposed electrode pairs stressed by $100 \mathrm{kV}$ to ground for testing a layer of insulation tape (Polyamide Kapton, $\varepsilon_{r}=7.0$ ) in air. There will be a very high electric field in the air, $757 \mathrm{kV} / \mathrm{mm}$ for this example which will lead to an extensive corona discharge around the electrode. The discharge can destroy the material at the edges and a puncture or flashover can happen outside the electrode area instead of in the middle.
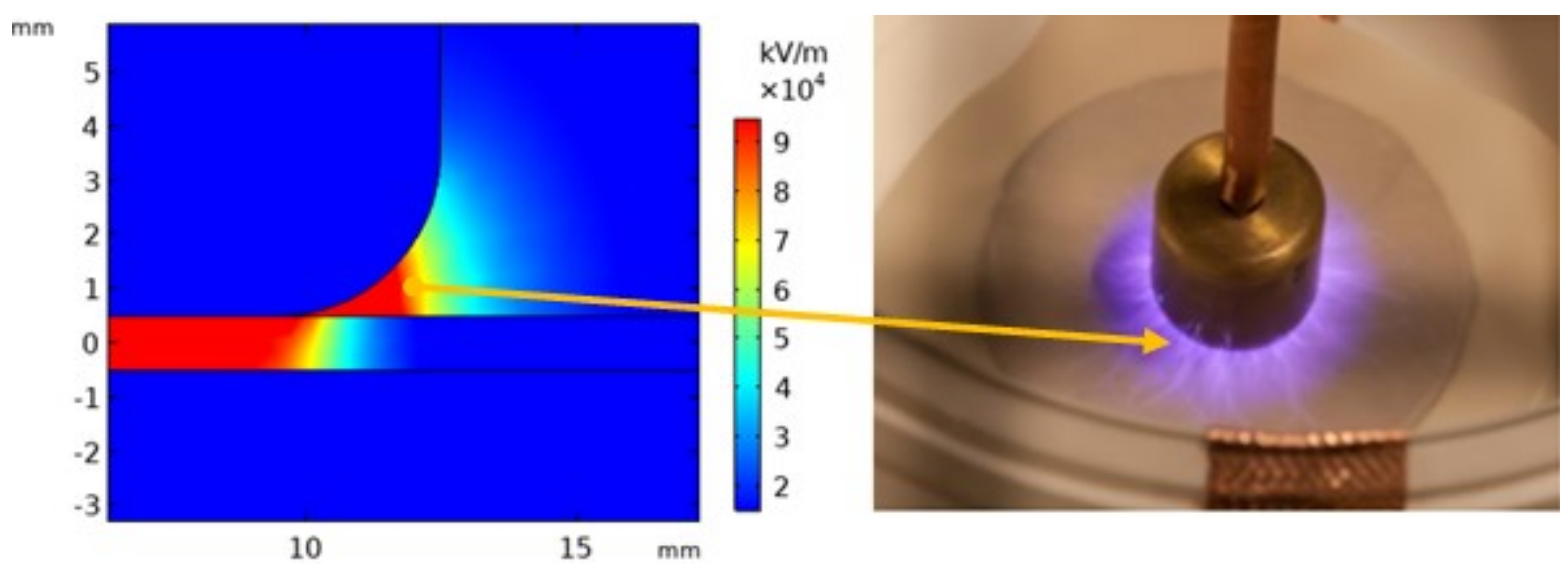

Figure 3-22. The effect of improper surrounding insulation material on the unwanted external discharges on the surface of the insulation material under test

Depending on the relative permittivity of the materials [65], the electrical field in the medium around a solid insulation material under a test can be higher than the electrical field within the test object. This causes surface discharge and partial discharges near the test object (see Figure 3-22) which deteriorates the material around the electrode corners and can affect the breakdown voltage of the test setup [105]. During application of voltage, by placing the sample inside a suitable oil as a surrounding medium, there will be no external discharge at the edges of the electrodes, and an ACBD will happen inside the insulation material any discharge over the surface of the sample will be avoided.

Mineral transformer oil (IEC 60296), organic ester fluid (IEC 61099) and silicone fluid (IEC 60836) is proposed in IEC 60243-1 among the other suitable fluids [98] as a selection for surrounding medium.

\subsection{High frequency withstand tests on the Litz wire}

In this section the withstand tests on the Polysol155 Litz wire used in this project is explained. A voltage supply has been made in a previous Master thesis project and is used here to perform the tests.

\subsubsection{Withstand test setup used for the project}

An IGBT full bridge converter was manufactured during a Master thesis defined by RISE and performed by students from Chalmers. The highest voltage available from the source is $\pm 350 V_{\text {peak- }}$ Peak. Figure 3-23 demonstrates the circuit diagram and specification of the converter. 


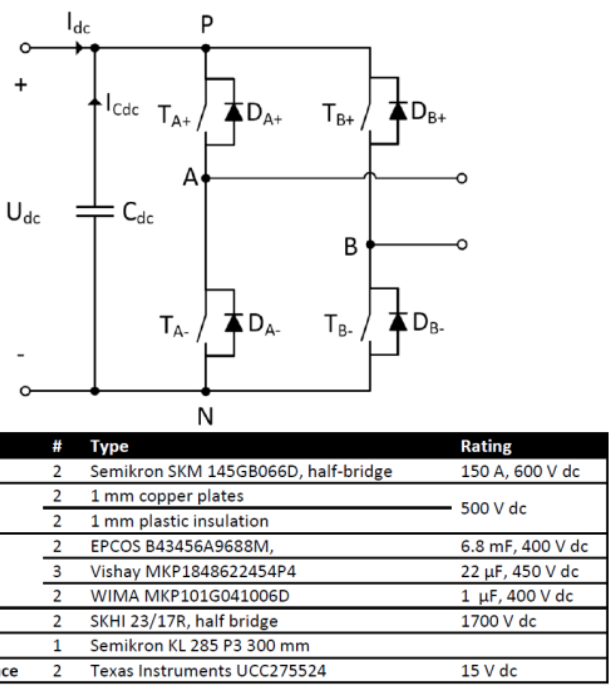

Figure 3-23. IGBT full bridge converter for production of square waves

Some points should be explained here:

- The ratio of step-up transformer is checked using an audio amplifier in the range of $50 \mathrm{~Hz}$ to $5 \mathrm{kHz}$. The measurement shows that the transformer has nearly the same ratio at this frequency range. If the applied voltage is a square wave signal it contains higher orders of voltage harmonics and the output voltage will not be a square wave signal but possibly more towards a voltage of near sinusoidal shape.

- The high voltage divider used should have a wide frequency range to truly transfer the wave shape at the high voltage primary to the scope's input.

- To do the insulation tests, it is not enough to have a high voltage source but there should be a current limiting facility to protect the converter every time the short circuit happens in the insulation material. The SKHI 23/12 (R) drive circuit for IGBTs is selected to have a short circuit turn-off protection by $\mathrm{V}_{\mathrm{CE}}$ monitoring.

The following equipment was used in the test setup:

- IGBT full bridge converter, $10 \mathrm{~kW}, \mathrm{DC}$ link $350 \mathrm{~V}$, maximum switching frequency $45 \mathrm{kHz}$

- HILO-test, HVT $240 \mathrm{R} / \mathrm{CR}$ wide frequency band (7.3 MHz), high voltage divider Ratio 12440:1

- Step-up transformer: $600 \mathrm{~V} / 22 \mathrm{kV}, 333 / 9.1 \mathrm{~A}$

- Pico, TA044, $70 \mathrm{MHz}$ High-voltage differential probe

The circuit diagram for the $5 \mathrm{kHz}$ insulation tests is presented in Figure 3-24 and the setup in Figure 3-25.

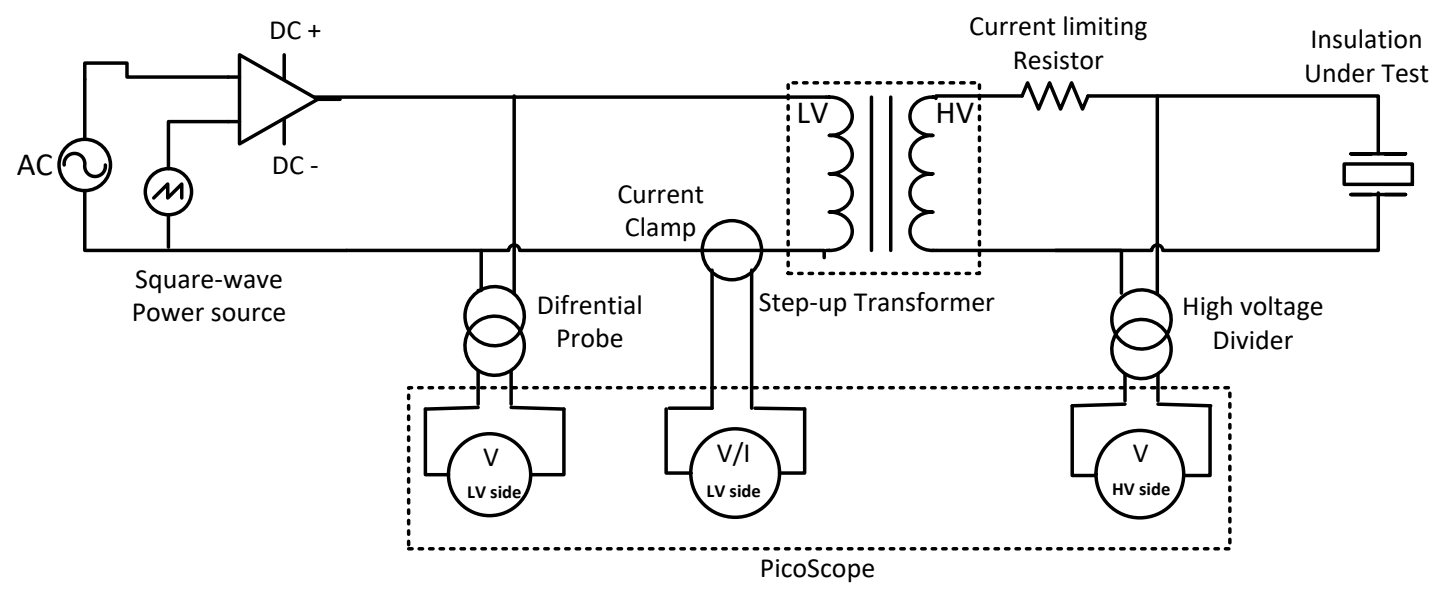

Figure 3-24. Circuit diagram for the $5 \mathrm{kHz}$ insulation tests. 

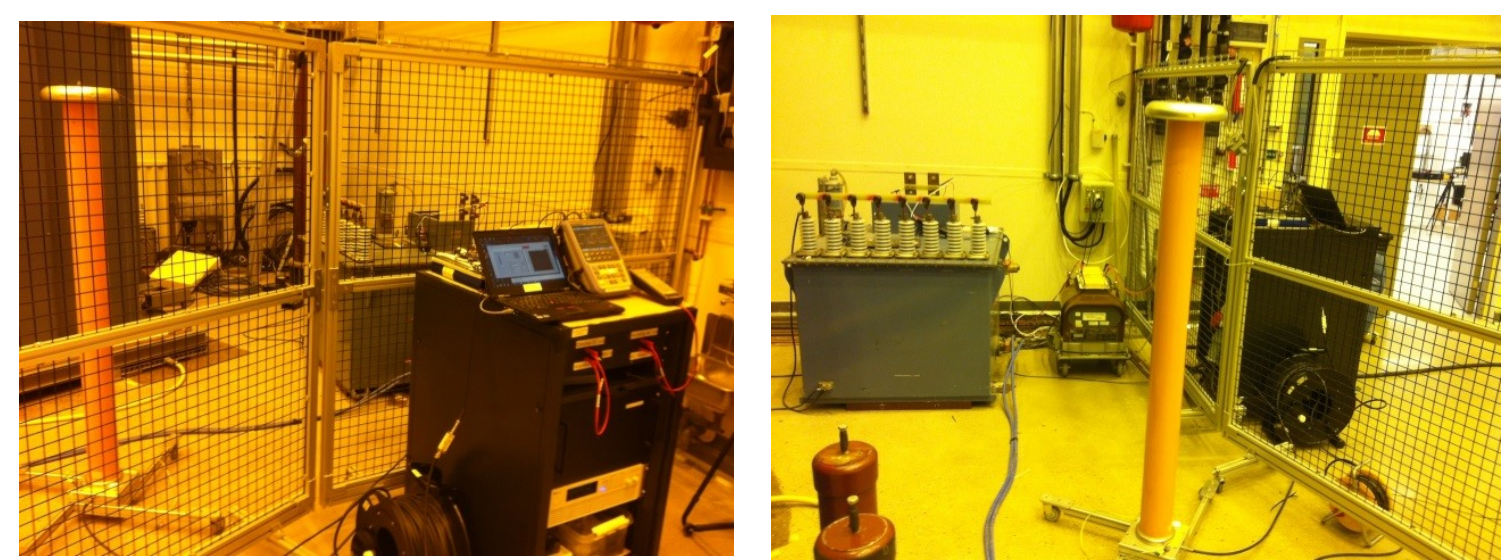

Figure 3-25. IGBT full bridge converter, step-up high voltage transformer and high voltage wide band voltage divider.

The test samples were prepared considering precautions given in IEC 60851-5 [96]. For each sample, two wire strands are twisted 33 times. A load of $0.85 \mathrm{~N}$ was applied during twisting the pairs. The rate of voltage increase was $100 \mathrm{~V} / \mathrm{sec}$. At least 5 test specimens were tested. Figure 3-26 shows the prepared samples according to precautions of IEC 60851-5.
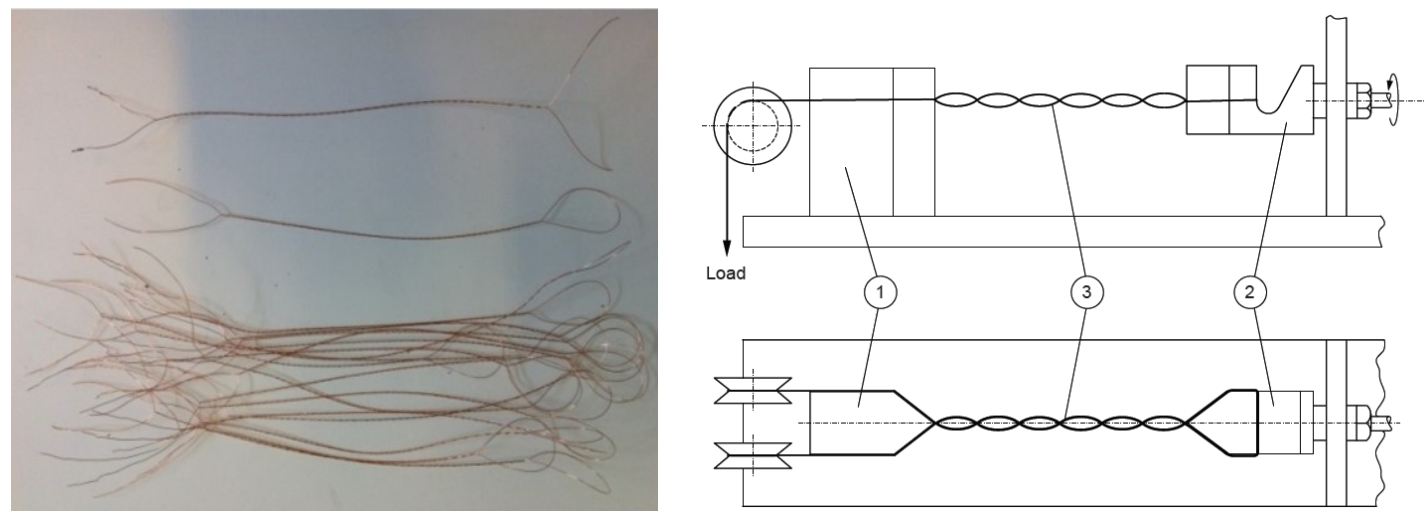

Figure 3-26. Insulated wire samples prepared according to precautions of IEC 60851-5

Unfortunately, $180 \mathrm{~V} / \mu \mathrm{m}$ is an Elektroisola's typical value for $0.25 \mathrm{~mm}$ Litz wire and no standard (IEC 60851-5 for example) is followed and mentioned in the product specification. 2.5-3.0 kV is stated from the producer side as the breakdown voltage of the wire to ground (This can be interpreted as nearly $5 \mathrm{kV} /$ wire-wire insulation). The results for all three types of the tests is presented in Table 3-3.

Table 3-3. Comparison of breakdown voltage at different frequencies on twisted strands of Litz wires

\begin{tabular}{|c|c|c|c|c|}
\hline & & DC & $\begin{array}{c}50 \text { Hz r.m.s. } \\
\text { sinus }\end{array}$ & $\begin{array}{c}5 \mathrm{kHz} \text { r.m.s. } \\
\text { sinus }\end{array}$ \\
\hline & & 6.9 & 3.5 & 1.2 \\
\hline & & 5.8 & 3.2 & 1.1 \\
\hline & & 4.6 & 2.9 & 1.1 \\
\hline & & 6.3 & 3.1 & 1.2 \\
\hline & & 6.2 & 3.4 & 1.1 \\
\hline & & 5.1 & 3.7 & 1.1 \\
\hline Information from the producer & Material Spec. & & & \\
\hline Wall thickness $\sim 73 \mu \mathrm{m} ; \sim 2.5-3.0 \mathrm{kV}$ & $180 \mathrm{~V} / \mu \mathrm{m}$ & $5.8 \mathrm{kV}$ & $3.3 \mathrm{kV}$ & $1.1 \mathrm{kV}$ \\
\hline
\end{tabular}

Compared with the value presented in the material's datasheet the following results can be presented:

- Nearly 5.8/5.0=1.2 times higher breakdown voltage for DC

- Nearly 5/3.3= $\mathbf{1 . 5}$ times lower breakdown voltage for $\mathbf{5 0 ~} \mathbf{~ H z}$

- Nearly 5/1.1= 4.6 times lower breakdown voltage for $5 \mathbf{~ k H z}$ sinus wave form 
The tests with $5 \mathrm{kHz}$ square wave voltage can show much lower breakdown voltage results. It is shown in Table 3-3 how an increase of the frequency from $50 \mathrm{~Hz}$ to $5 \mathrm{kHz}$ causes the withstand ability of the insulation material to be reduced from $5.8 \mathrm{KV}$ under a DC excitation to $1.1 \mathrm{kV}$ under a $5 \mathrm{kHz}$ stress. The frequency spectrum of a square wave signal contains frequencies which are much higher than $5 \mathrm{kHz}$. As explained in this section, the higher the frequency, the higher the stress on the insulation material. This is a subject of future research.

The rate of voltage-increase and the time of application of a fixed voltage before reaching the breakdown voltage will affect the final recorded voltage at the time of breakdown. Partial discharge starts at a lower voltage level than the breakdown voltage. The corona sound was clearly noticeable even where the voltage was one third of the breakdown voltage. The discharge destroys the wires' insulation in the long run. Many efforts have been done to produce corona resistive motor winding wires [106], however corona resistivity does not mean corona free. The lifetime will be reduced anyhow. It is suggested to measure the partial discharge inception voltage for the actual conductorinsulation-ground physical combination. Insulation system models should be based on transformer design and the actual ambient condition. PD inception levels should be measured, and the transformer insulation design should be based on critical voltage obtained from the experiment. The designed transformer should not experience partial discharge at its rated operating condition.

In order to demonstrate the effect of application of a standard method (IEC 60851-5) compared with a simulated test procedure (presented earlier), a second series of tests were performed.

The samples were $25 \mathrm{~cm}$ long twisted Litz wires. The voltage is applied between two bunches of twisted wires. The results are summarized in Table 3-4.

Table 3-4. Comparison of breakdown voltage at different frequencies on $25 \mathrm{~cm}$ of twisted Litz wires

\begin{tabular}{|c|c|c|c|}
\hline & DC & $50 \mathrm{~Hz}$ sinus & $5 \mathrm{kHz}$ sinus \\
\hline \hline & 2.9 & 0.8 & 0.90 \\
\hline & 1.9 & 0.4 & 0.92 \\
\hline & 2.6 & 1.3 & 0.91 \\
\hline & 3.0 & 1.3 & 0.89 \\
\hline & 3.4 & 0.8 & 0.90 \\
\hline Maximum & 4.1 & 2.0 & 0.92 \\
\hline Minimum & 1.6 & 0.4 & 0.89 \\
\hline Average & $2.9 \mathrm{kV}$ & $0.8 \mathrm{kV}$ & $0.9 \mathrm{kV}$ \\
\hline
\end{tabular}

Both series of results, obtained from a standard test and a simulated test, show that in a typical prototype transformer, the breakdown voltage can be several times lower than the figure presented in the material's technical specification. It is suggested to do insulation tests on the simulated conductor-insulation-ground test setups, before attempting to start designing prototype transformers. 


\section{Prototype HVDC MFT design}

One the most serious attempts reported in literature for the insulation design of a prototype power electronic transformer is reported by Jaritz et. al [9]. He has reported the design and manufacturing of a 0.4/14.4 kV MFT with $115 \mathrm{kV}$ insulation at its HV side to ground for the new linear collider at the European Spallation Source (ESS) in Lund (2.88 MW pulse modulators with pulsed output voltages of $115 \mathrm{kV})$. However, all the insulation design is for an AC stress and only based on a $\mathrm{kV} / \mathrm{mm}$ high voltage strength and the primitivity of the insulation materials values of the material used. Plastic materials in combination with synthetic ester are used for the insulation and a final AC insulation test is performed inside an oil tank. The oil used, has a high conductivity value which is in combination with a very low conductivity value of the plastic materials used which can result in a very uneven electrical field distribution under a high voltage DC stress. No report on a study on compatibility of the plastics in the oil is reported.

Bahmani [3] is presented the design of a $50 \mathrm{~kW}, 1 / 3 \mathrm{kV}, 5 \mathrm{kHz}$ dry type transformer. The insulation design was based on a $\mathrm{kV} / \mathrm{mm}$ high voltage strength values of the insulation materials used as coilformer and to guarantee a safe insulation design a safety factor of 2 was considered. In some places of the transformer a non-uniform electric field exists that possibly will not be covered, even with the using a high safety factor.

As a contribution, in this work a real DC insulation design concept is implemented which results in a more compact design (using actual voltage and temperature based conductivities instead of an application of permittivity based AC insulation design) as well as improving the reliability of the highvoltage design (avoiding simplified $\mathrm{kV} / \mathrm{mm}$ values for the sophisticated design procedure and eliminating the need for using a large safety factor). In addition, other parameters like partial discharge inception voltage (which is not avoidable in dry type transformers), insulation design for multi-layer insulation systems (which cannot be solved simply by adding $\mathrm{kV} / \mathrm{mm}$ figures of the layers), non-uniform electric fields (at the corners of the design) and the effect of frequency on the ignition and development of discharges (which are not considered in the $50 \mathrm{~Hz}$ reference breakdown voltage values) are also considered. Finally, the behaviour of the transformer is studied under HVDC stress.

As, the main task in this project is to present a reliable design method for a high HVDC insulation to ground, it was decided to design and manufacture one prototype transformer and based on the results, give guidelines for the design of an industrial scale transformer. Therefore, having an eye on the Jaritz's and Bahmani's contributions, and to go a step further, the following case is selected as the main target as prototype for verification of the design methodologies developed in this project:

A prototype transformer of $50 \mathrm{~kW}, 0.4 / 5 \mathrm{kV}, 5 \mathrm{kHz}$ with the HVDC insulation to ground of $125 \mathrm{kV}$

\subsection{Prototype magnetic design}

The transformer design starts with having the following basic design input:

- $\quad$ Output power, $P_{\text {out }}=50 \mathrm{~kW}$

- (The same with two previous prototypes and adopted to the available California Instrument $4500 \mathrm{LX} \mathrm{AC,} 50 \mathrm{~kW}, 5 \mathrm{kHz}, 150 / 300 \mathrm{~V}$ single/three phase PWM converter)

- Voltage ratio, $V_{L V} / V_{H V}=0.42 / 4.5 \mathrm{kV} / \mathrm{kV}$ 
- (The primary voltage adopted to the DAB bridge that will be available to connect the prototype transformer to it and the secondary voltage as an integer multiple of the primary voltage)

- Switching frequency, $f=5 \mathrm{kHz}$

- (According to [107] the highest power density of samples of medium frequency transformers are achieved at a frequency between $10-25 \mathrm{kHz}$. As the available California Instrument $4500 \mathrm{LX}$ source can only reach $5 \mathrm{kHz}$, this frequency is selected for the prototype transformer design)

- Duty cycle $D=0.5$ and relative rise time $R=0$

$\circ$ (see Figure 2-1a)

- Isolation Level, $V_{\text {iso }}=125 \mathrm{kV} \mathrm{DC}$

$\circ$ (The high voltage or secondary winding side DC insulation to ground)

The following design was used from the basic design idea using the equations earlier mentioned in section 2.4):

- Rated primary current, $I_{T 1}($ r.m.s. $)=50 \mathrm{~kW} / 421.8 \mathrm{~V}=118.5 \mathrm{~A}$

- Rated secondary current, $I_{T 2}$ (r.m.s.) $=50 \mathrm{~kW} / 4500 \mathrm{~V}=11.11 \mathrm{~A}$

- Maximum allowed current density, $J_{\max }=3 \mathrm{~A} / \mathrm{mm}^{2}$

- (According to Litz wire manufacturer's datasheet for operation at $95^{\circ} \mathrm{C}$ )

- Number of turns, $N_{1}$ and $N_{2}=12$ and 128

- (As shown in Figure 4-2 in this way the voltage difference between two adjacent discs of the high voltage winding will be $4.5 \mathrm{kV} / 128 \times 16=560 \mathrm{~V}$ which is less than $0.9 \mathrm{kV}$ and safe enough according to the experimental verification tests presented in section 3.5.1)

- Magnetic core cross section, from (2-32) calculated as $5493 \mathrm{~mm}^{2}$ for Ferrite where

- $V_{r m s 1}$ is the rated voltage of the winding $=421.8 \mathrm{~V}$

- $K_{c}$ is the core filling factor $\approx 1$ for Ferrite

- ( $k_{c}$ is the core filling factor or the ratio of effective to physical cross sections)

- $\quad N_{1}$ is the number of turns of the primary winding $=12$

- $B_{m}$ is the maximum working flux density of the core $\approx 0.32 \mathrm{~T}$ for Ferrite

○ $\quad(\approx 80 \%$ of the saturation flux density [83])

- $f$ is the switching frequency $=5 \mathrm{kHz}$

- A core is selected having a core window of highest dimensions achievable. The commercially available sample Epcos TDK type B67385U 126/91/20 (see Figure 4-1) is selected which has $20 \times 28=560 \mathrm{~mm}^{2}$ as physical core area. This means that 10 core stacks including $20 \mathrm{U}$ cores is suitable for the design. The maximum working flux density of the core will be $0.314 \mathrm{~T}$.
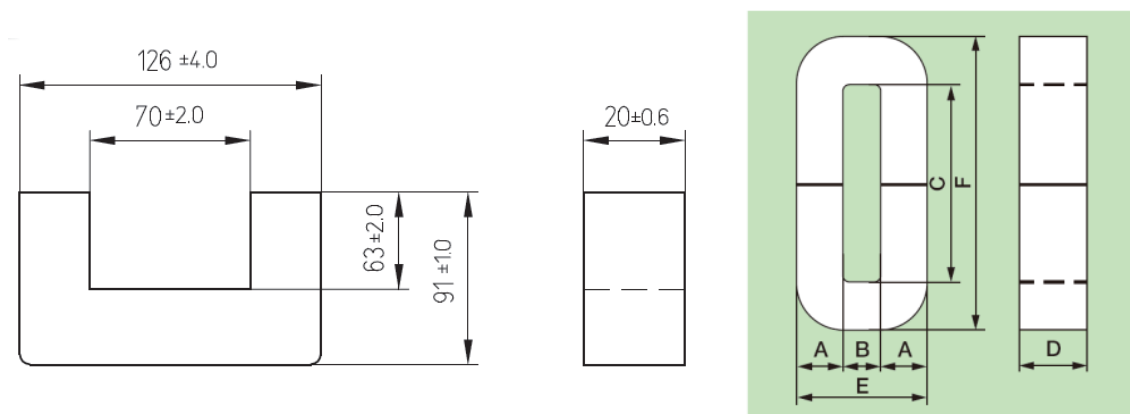

Dimension

$\mathrm{mm}$

$A=28 \pm 1$

$B=70 \pm 2$

$\mathrm{C}=126 \pm 4$

$\mathrm{D}=20 \pm 0.6$

$\mathrm{E}=126 \pm 4$

$\mathrm{F}=182 \pm 2$

Figure 4-1. Dimensions of an Off the shelf Ferrite core which is used for HVDC MFT prototype 
- For the primary winding, considering $118.5 \mathrm{~A}$ as rated r.m.s. current, the space that the primary winding occupy in the core window will have a height of $h_{w 1}=3.8\left(h_{b 1}\right) \times 8\left(n_{p 1}\right) \times 4$ $\left(N_{11}+1\right)=121.6 \mathrm{~mm}$ (Layer type winding using the Litz wire introduced in section 6.1.3) and the width of $W_{1}=2.5\left(w_{b 1}\right) \times 4\left(m_{1}\right)=10 \mathrm{~mm}$ where $h_{w 1}$ is the primary winding height, $h_{b 1}$ is the Litz wire height used for the primary winding,$n_{p 1}$ is the number of parallel Litz wires of the primary winding, $N_{11}$ is the number of Litz wires per layer of the primary winding, $W_{1}$ is the width of the primary winding, $w_{b 1}$ is the Litz wire width and $m_{1}$ is the number of layers of primary winding (see Figure 4-2). Note that the ideal magnetic height of the winding will be $3.8\left(h_{b 1}\right) \times 8\left(n_{\mathrm{p} 1}\right) \times 3\left(N_{11}\right)=91.2 \mathrm{~mm}$. From the manufacturer's datasheet for the $181 \times 0.2$ $\mathrm{mm}$ Litz wire, the maximum current density is $3 \mathrm{~A} / \mathrm{mm}^{2}$ and the filling factor is 0.6 . The actual current density will be $2.6 \mathrm{~A} / \mathrm{mm}^{2}$.

- For the secondary winding, considering $11.11 \mathrm{~A}$ as rated r.m.s. current, $3 \mathrm{~A} / \mathrm{mm}^{2}$ as maximum current density and 0.6 as filling factor of the Litz wire, the space that the secondary winding occupy in the core window will have a height of $h_{w 2}=3.8\left(h_{b 2}\right) \times 16\left(N_{12}\right)=$ $60.8 \mathrm{~mm}$ (Disc type winding) and the width of $W_{2}=2.5\left(w_{b 2}\right) \times 8\left(m_{2}\right)=20 \mathrm{~mm}$ where $h_{w 2}$ is the secondary winding height, $h_{b 2}$ is the Litz wire height used for the secondary winding , $N_{12}$ is the number of Litz wires per layer of the secondary winding, $W_{2}$ is the width of the secondary winding, $w_{b 2}$ is the Litz wire width and $m_{2}$ is the number of layers of the secondary winding ( see Figure 4-2). The actual current density will be $x 1.95 \mathrm{~A} / \mathrm{mm}^{2}$.

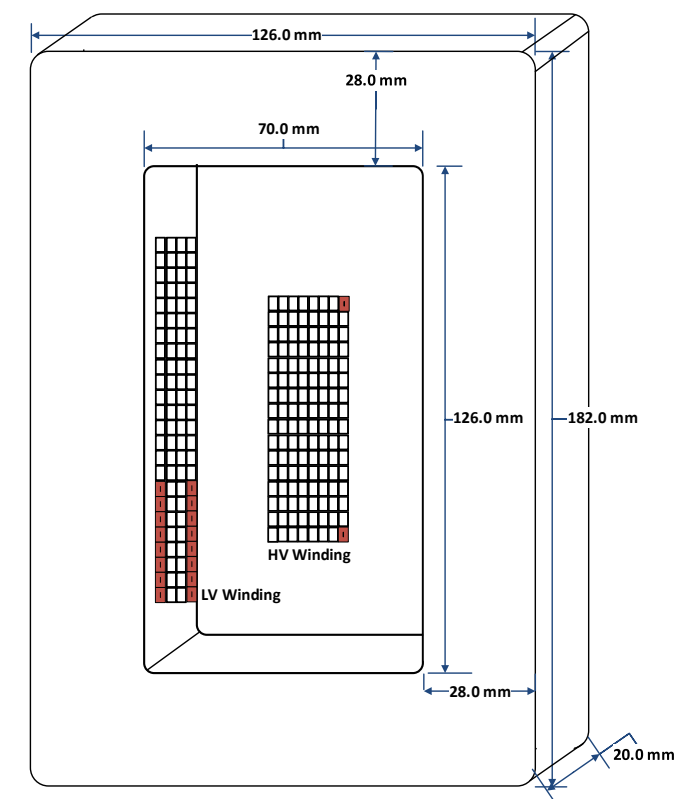

(a)

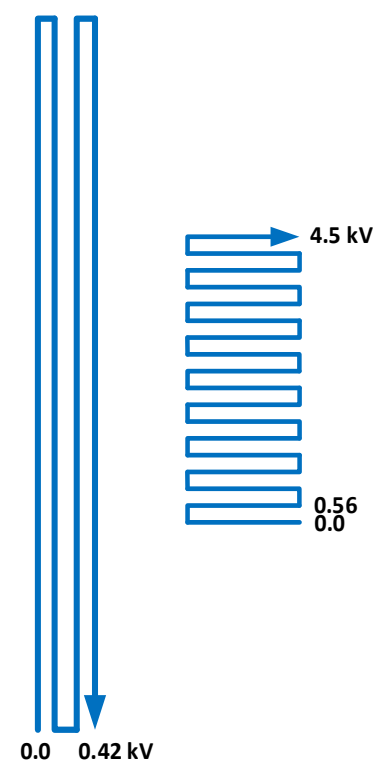

(b)

Figure 4-2. Windings in the core window and their connection type; The position of the windings in the core window (a), Connection type of the windings (b)

Before continuing with the other important magnetic design aspects which will be covered in section 4.3 it is necessary to be sure that the insulation of the transformer is safely designed to withstand a predetermined HVDC stress between the HV winding and the other conducting parts of the active part. 


\subsection{Prototype insulation design}

The insulation design is mainly focused on the isolation of the high voltage (secondary) winding in the $70 \times 12 \mathrm{~mm}^{2}$ core window. The core is grounded and the LV (primary) winding will be stressed only with $0.4 \mathrm{kV}$ to ground which can be assumed as grounded focusing on the very high $125 \mathrm{kV}$ DC which is going to be applied to the HV winding. The transformer tank is of a plastic cylinder which has a suitable electrical insulation property. Therefore, considering the windings' dimensions in the core window, the main task is to check if the winding-core clearance is enough to fulfil the DC insulation criteria if a step of $125 \mathrm{kV}$ DC voltage is applied.

A Non-Linear Maxwell-Wagner (NLMW) model is adopted for the MFT insulation design, which accounts for (at least) field and temperature dependencies of the electric conductivity of the constituting materials.

The $50 \mathrm{~kW}$ MFT operates at biased 422/4500 V/V AC (5 kHz) and $125 \mathrm{kV}$ DC voltages as mentioned earlier in the introduction to the section 4). The insulation system is built based on mineral transformer oil and Oil-Impregnated Pressboard (OIP) to fulfil the requirement of high DC voltage isolation to ground and a stabilized temperature condition.

To minimize the number of sharp corners and to provide a better heat transfer between the windings and the core stacks, a polygonal design is considered (Figure 4-3) instead of the previously used planar designs [8]. This structure allows for installing OIP barriers to achieve a high voltage withstand level in a minimum space. Justifications of the OIP thickness and oil gaps widths were performed by considering the withstand levels of the overall dielectric structure obtained from the FEM simulation. Precautions were made to achieve oil gaps of the same size to let a smooth flow of cooling oil through the entire insulation system.

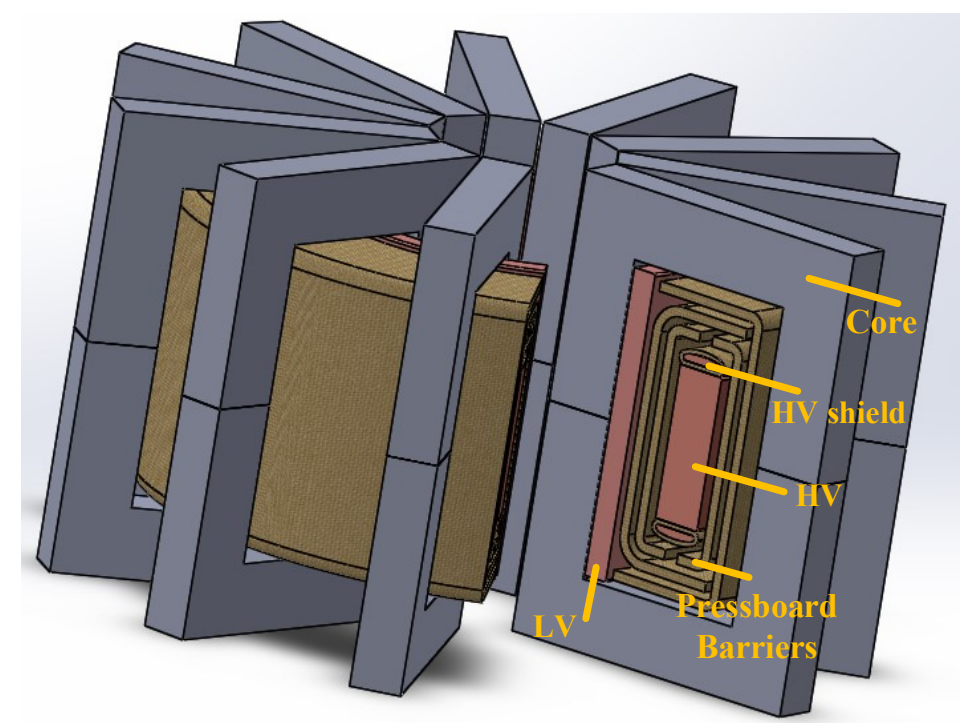

Figure 4-3. Polygonal transformer design

The NLMW model outlined in section 2.2.11.4 was utilized to estimate the withstand levels of the insulation system of the MFT prototype. The transformer geometry including its insulation system was implemented in COMSOL Multiphysics (LiveLink to MATLAB was used). The governing equations 2-8) to (2-12) were implemented using respective modules in the software. The measured temperature and field dependent conductivities of oil and OIP presented in section 3.3 were utilized to establish the NLMW model. A set of simulations was conducted for optimizing the geometry of the 
insulating barriers. The performance of each geometry was evaluated at temperatures 30,50 and $90^{\circ} \mathrm{C}$.

The calculations were performed assuming axisymmetric conditions that is rather close to the case when a single section of the core is considered. Time domain analysis was implemented to find the stresses at different time steps starting from the instant of applying a step DC voltage $(1 \mathrm{~s})$ to the final steady state (at $10^{4}$ seconds). Figure 4-4 demonstrates the potential distribution and electric field on the insulation materials for the moment of application of voltage and after a long time.
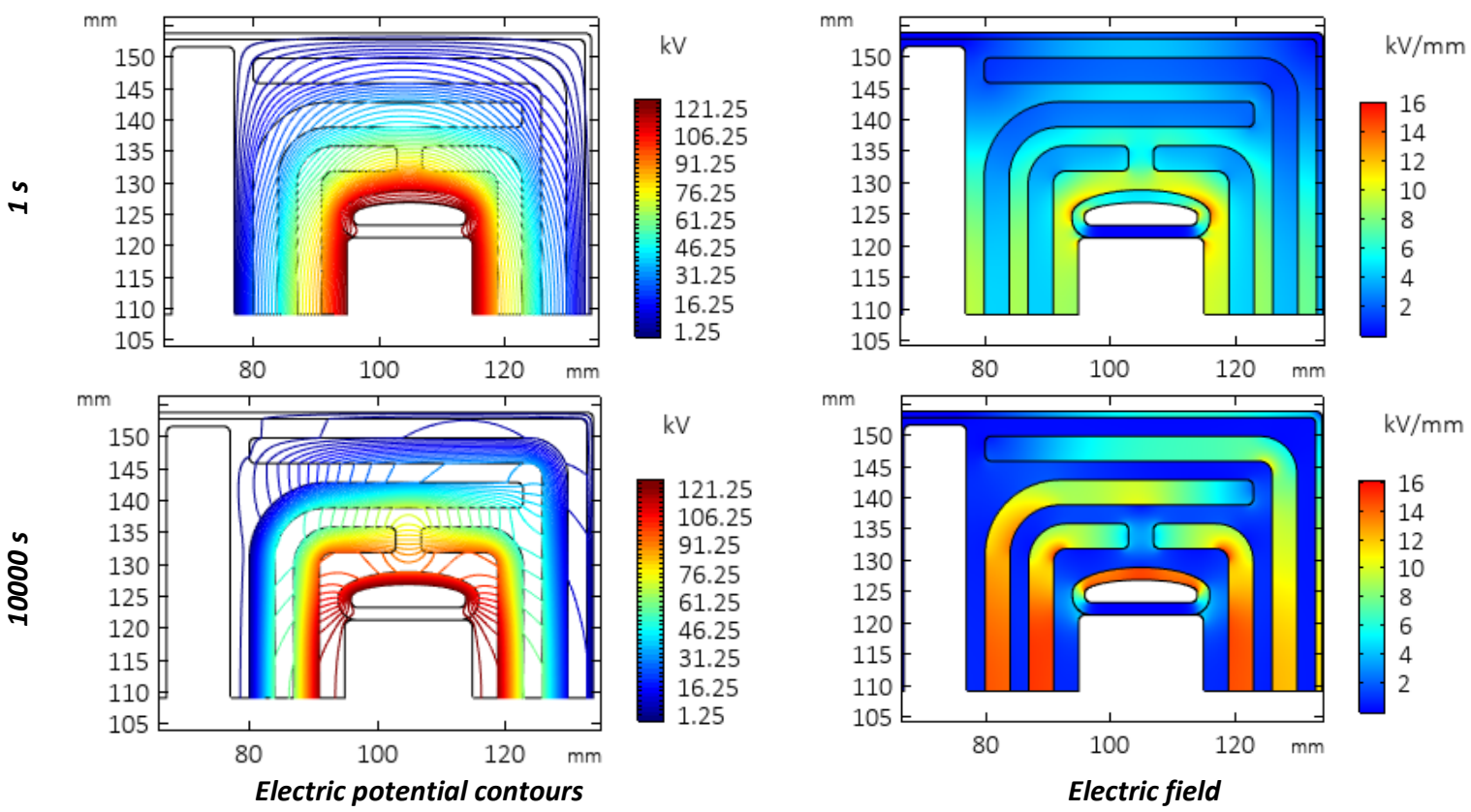

Figure 4-4. Electric potential and field distribution at 1 and 10000 seconds in the oil/OIP system

As an example, the electric field distributions in the cross-section of the winding at $50^{\circ} \mathrm{C}$ at 1,60 and 3600 seconds are shown in Figure 4-5. As expected, the results indicate clearly the high stress in the oil gaps at $1 \mathrm{~s}$, which is gradually transferred to the OIP with time (compare with the plot for 1 hour).

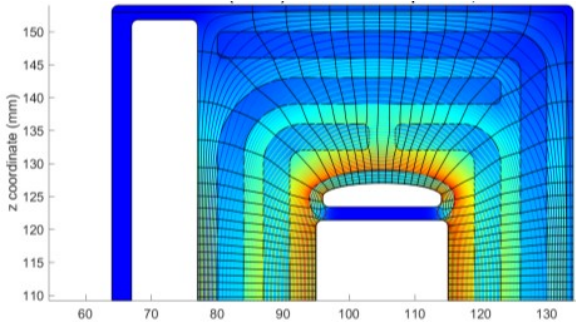

(a) One second

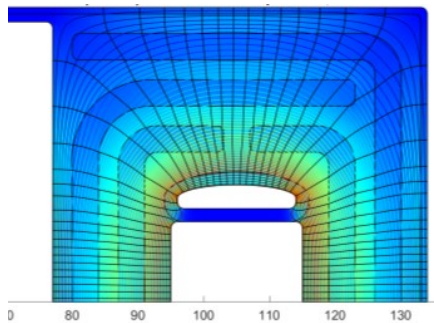

(b) One minute

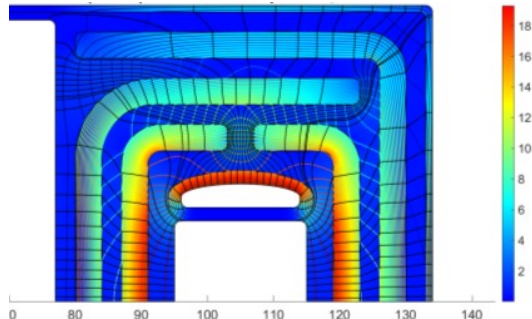

(c) One hour

Figure 4-5. Time domain analysis, electrical stresses at discrete time steps applying a step DC voltage. Surface: Electric field norm $(\mathrm{kV} / \mathrm{mm})$, Counter: Electric field potential, Streamline: Electric field

The time variation of the field strength at two points at the corner of the HV shield (one in the OIP and the other in the adjacent oil gap) is shown in Figure 4-6. The stress at the point in the OIP, has decreased after experiencing a clear maximum at about 950 seconds. Similar analysis of the electrical stress in the oil gaps, OIP barriers and along creepage paths has been performed. 

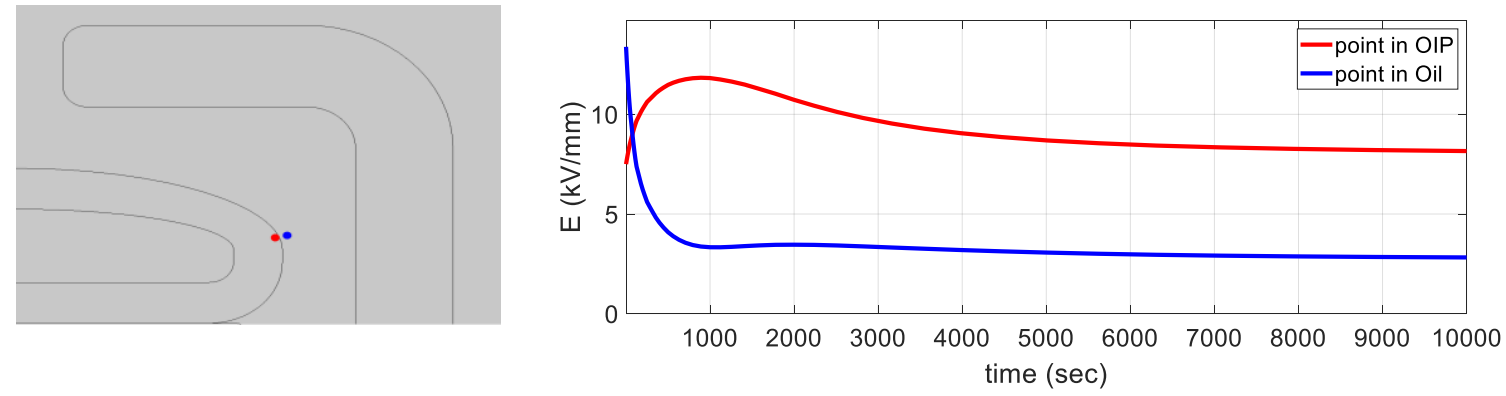

Figure 4-6. The time variation of the field strength at two points at the corner of the HV shield

The dynamic behaviour is characterized by the time constants $\tau_{\text {oil }}=\varepsilon_{\text {Oil }} / \sigma_{\text {Oil }}$ and $\tau_{\text {OIP }}=\varepsilon_{\text {OIP }} / \sigma_{\text {OIP }}$. The relative dielectric permittivity of the materials can be assumed to be constant at the operational conditions of the transformer and equal to typical values of 2.2 and 4.4 for oil and OIP, respectively [66]. In contrast, the electric conductivities depend on temperature and electric stress as demonstrated in this work. According to Table 3-1, to cover all possible combinations of oil and OIP conductivity ratios, three different conditions were considered, namely, $C R=294$ for $30^{\circ} \mathrm{C}, C R=29$ for $50^{\circ} \mathrm{C}, C R=1$ for $90^{\circ} \mathrm{C}$.

Seven different critical points were selected to represent the time dependent stress under these three conditions (see Figure 4-7).

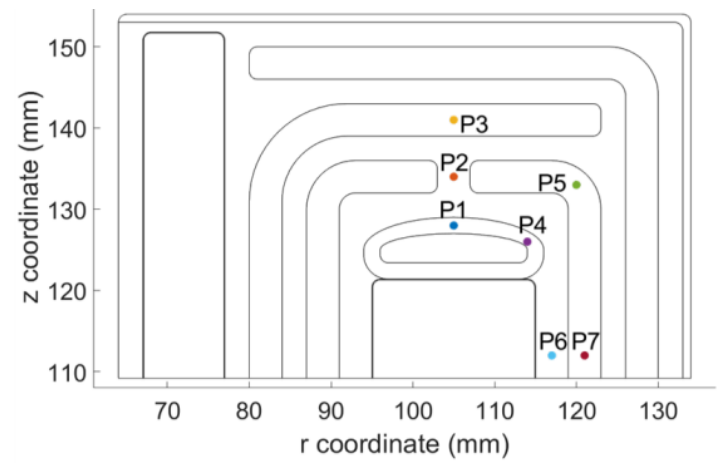

Figure 4-7. The geometry of the transformer and the points in which the electric field are obtained

As shown in Figure $4-8$, the time to reach to steady state at $30{ }^{\circ} \mathrm{C}$ with $C R=294$ is about $10^{5}$ seconds which is much longer than the stabilization time for $50{ }^{\circ} \mathrm{C}$ with $C R=29$. Meanwhile, the time for $90{ }^{\circ} \mathrm{C}$ with $C R=1$ is very short. In addition, a double exponential curve with a clear maximum occurs for both P1 and P4, which reveals that considering only the initial and final stress distribution is not enough for a practical insulation design method and such local maximums must be investigated carefully and limited as much as possible. The stresses on P6 and P7 at the lateral side of the HV winding indicate the stabilizing time of about $10^{4}$ seconds for 30 and $50^{\circ} \mathrm{C}$. However, this time span is much shorter than the 300 second value found for $90{ }^{\circ} \mathrm{C}$. A single exponential stress variation can be observed for both locations. 

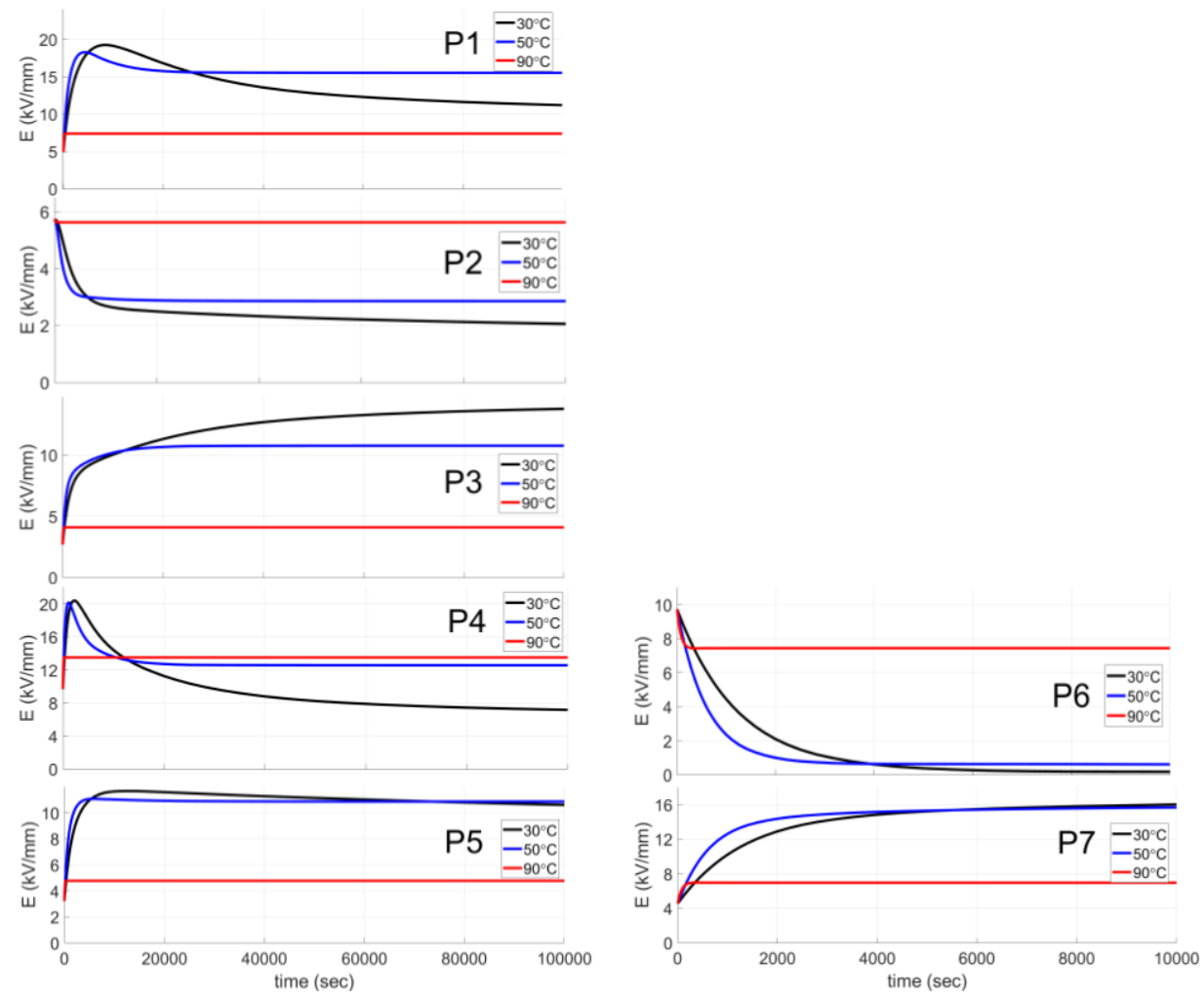

Figure 4-8. The electric field at P1 to P7 at different temperatures

\subsubsection{Oil gaps and OIP barriers design}

To identify the safety factor for each oil gap as well as the maximum stress at each OIP barrier, electric field streamlines originating from the points with the highest electric field in the transformer were utilized (Figure 4-9a). The field strength was obtained for every single streamline on each time step. Based on the streamline shown in Figure 4-9a by a thick red line, the field strength along the streamline is shown in Figure 4-9b. The variations of the values of $E_{b d}, E_{\text {sorted }}$ and $E_{a v}$ are presented in Figure 4-9c. Further, the safety factors for the oil gaps were calculated using (2-2) to (2-4) and the $S F$ curves of the oil gaps and presented in Figure 4-9d.

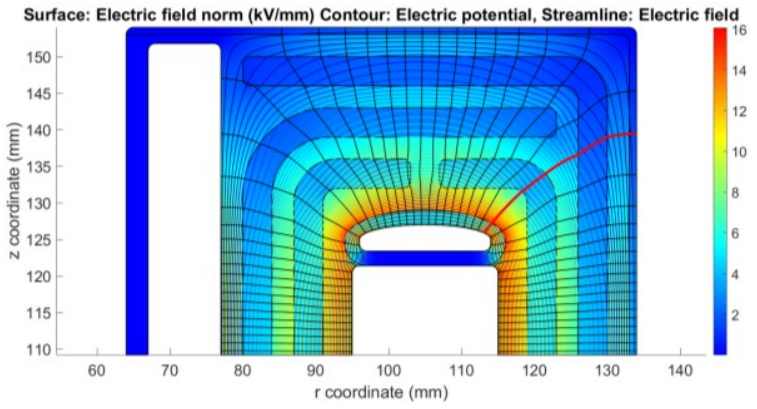

(a)

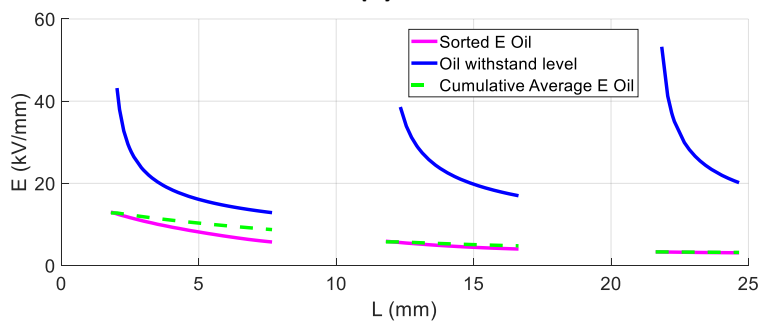

(c)

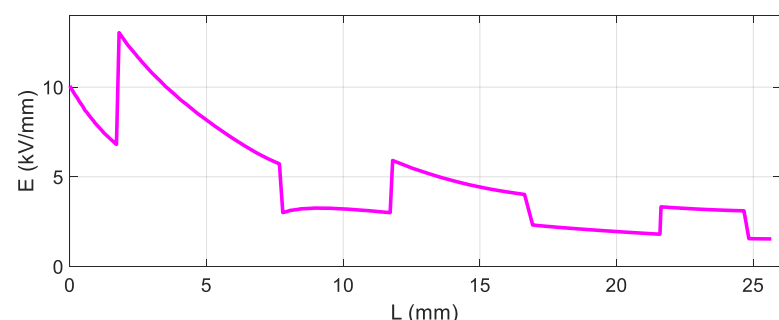

(b)

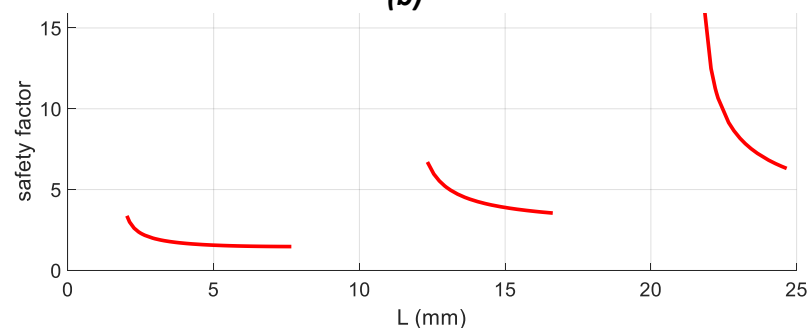

(d)

Figure 4-9. A sample simulation at $125 \mathrm{kV}, 50^{\circ} \mathrm{C}$; Streamlines originating from the points with the highest electric field and a red sample streamline $(a)$, The field strength along the red streamline $(b)$, The sorted stress $E_{\text {sorted, }}$ cumulatively integrated $E_{a v}$ curves compared with gap withstand curves $E_{b d}(c)$, Safety curves $S F(d)$ 
For the insulation design of conventional AC transformers, the safety factor of the oil gaps is considered to be $S F>1.0$ [65]. In this case, dependencies of the electrical conductivity of oil and OIP on moisture content, electrode materials and their coatings shall also be considered. In addition, as already explained in section 2.2.10, the electric field distribution in the HVDC oil-paper insulation systems does not fully comply with the LMW model. For these reasons, higher safety factors are considered as acceptable in this study for the DC insulation design. For the same reason, for the paths in the $4 \mathrm{~mm}$ OIPs the maximum stress of $20 \mathrm{kVr}$.m.s./mm are considered as critical [63].

A MATLAB script has been developed to determine the oil gaps with low safety factors and OIP barriers with high stress for further repetitive manual optimization of the design. For example, in Figure 4-10 the critical regions of the oil gaps and OIPs for all time steps are presented for the applied voltage of $125 \mathrm{kV}$. It should be mentioned that for all time steps at temperatures $30^{\circ} \mathrm{C}, 50^{\circ} \mathrm{C}$ and $90^{\circ} \mathrm{C}$, the minimum safety factor reached 1.67 in the oil gaps and the maximum local stress in the nonuniform field can be as high as $27.3 \mathrm{kV} / \mathrm{mm}$ in the OIP.

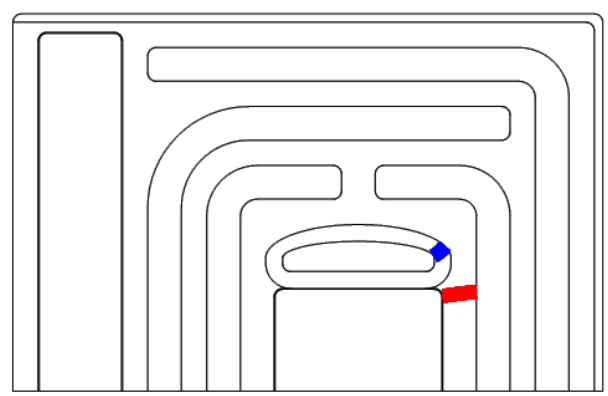

Figure 4-10. Critical regions for all the time steps at applied voltage $125 \mathrm{kV}$ are shown for oil gaps (red) with minimum SF and OIP barriers with maximum stress (blue) for temperatures 30,50 and $90^{\circ} \mathrm{C}$

\subsubsection{Interface insulation design}

To identify the safety factor for the oil-OIP interfaces, the tangential electric field on the existing creepage paths at all the time steps are considered. Figure 4-11 presents a summary of the simulations of the fields along a sample oil-OIP interface at $125 \mathrm{kV}, 50^{\circ} \mathrm{C}$ and 1 second performed with the NLMW model.

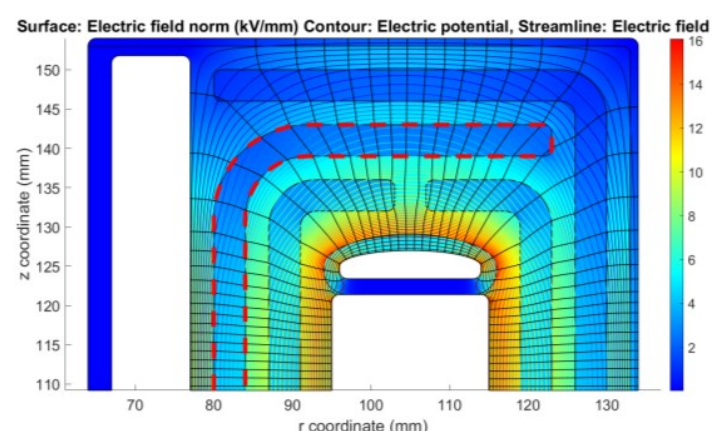

(a)

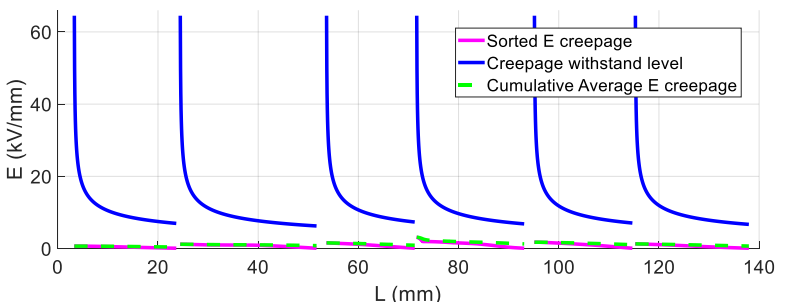

(c)

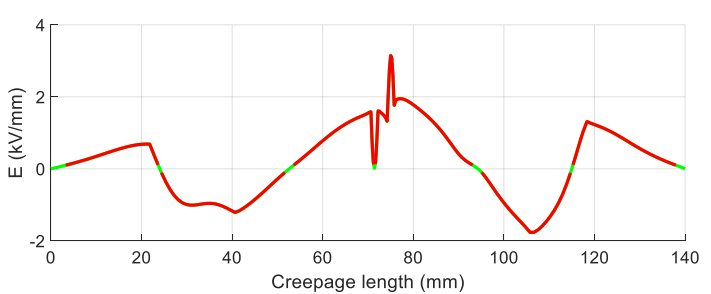

(b)

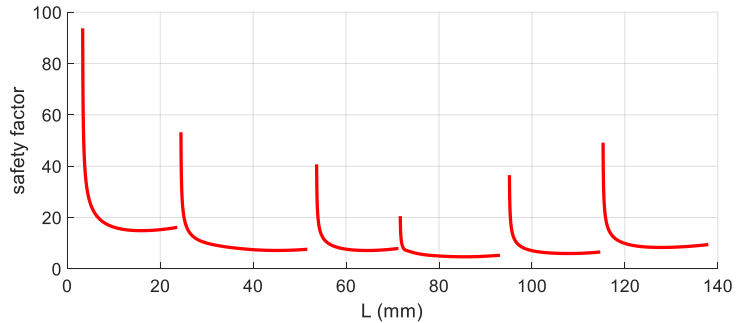

(d)

Figure 4-11. A sample simulation at $125 \mathrm{kV}, 50^{\circ} \mathrm{C}$ and 1 second; a sample oil-OIP interface (dashed red) (a), E-field along the creepage path (b), $E_{\text {sorted, }}$ cumulatively integrated $E_{a v}$ and $E_{b d}$ curves (c), Safety curves $S F(d)$ 
The selected interface is highlighted by a red dashed line for further illustrations (Figure 4-11a). The electric field along the displayed oil/OIP interface (Figure 4-11b) shows how the tangential field changes from positive to negative and drops below $0.1 \mathrm{kV} / \mathrm{mm}$ at some locations (green pieces along the curve). It is assumed that when the tangential field drops under this value, the creepage path is discontinued, and a new path starts as soon as the field reaches a stress higher than $0.1 \mathrm{kV} / \mathrm{mm}$ [22]. In such a way, the abovementioned interface is cut to pieces and, for each piece, the $E_{b d}, E_{a v}$ and sorted $E_{\text {sorted }}$ curves are displayed in Figure $4-11 \mathrm{c}$ by blue, green and pink curves, respectively. It is worth mentioning that the creepage withstand $E_{b d}$ curves are assumed to be 0.7 times of the withstand level of an oil gap of the same path length [65], which is presented in (2-3). The creepage paths safety factor curves (Figure 4-11d) are calculated by dividing the withstand curves to the cumulative average curves. The safety factor curves have been considered for temperatures 30,50 and $90^{\circ} \mathrm{C}$, and for all time steps from the time of energizing the transformer until $10^{4}$ seconds. The minimum safety factor for all time steps and all temperatures was 1.55. An output from the simulation demonstrating the critical creepage paths is presented in Figure 4-12, where the red lines indicate safety factors less than 1.6.

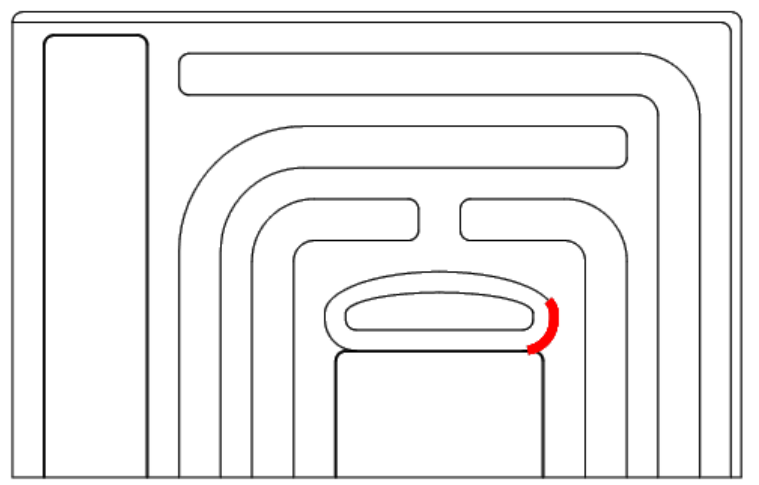

Figure 4-12. The critical paths for all time steps with minimum SF shown in red for temperatures 30,50 and $90^{\circ} \mathrm{C}$

\subsection{Prototype magnetic design continued}

As mentioned earlier in section 2.3.1, three important design aspects are Leakage inductance, core losses and winding losses.

\subsubsection{Leakage inductance}

To calculate the leakage inductance of the Decagon MFT (the prototype MFT which has a decagonal shape), FEM simulations were performed using the free available software FEMM4.2 [108]. The internal walls of the core window are replaced by Neumann boundary conditions $(\partial A / \partial n=0)$. Figure 4-13 shows flux distributions inside the core window and a simulation for the winding parts outside of the core window obtained by FEM analysis. 


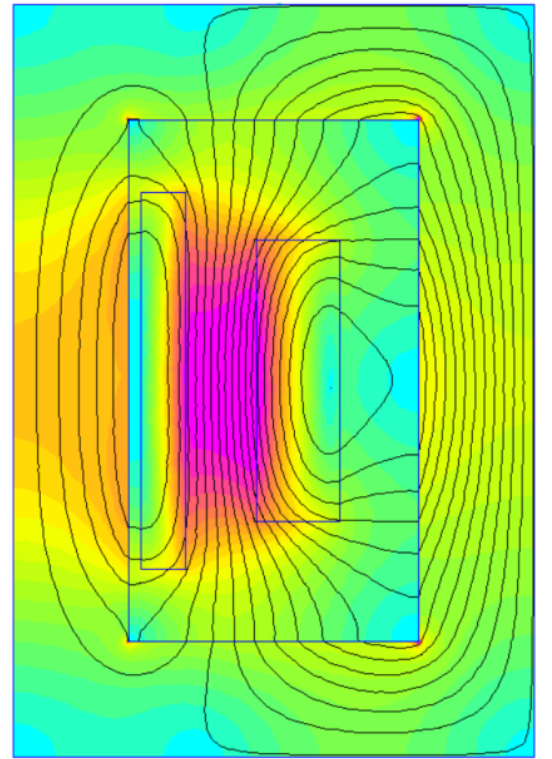

(a)

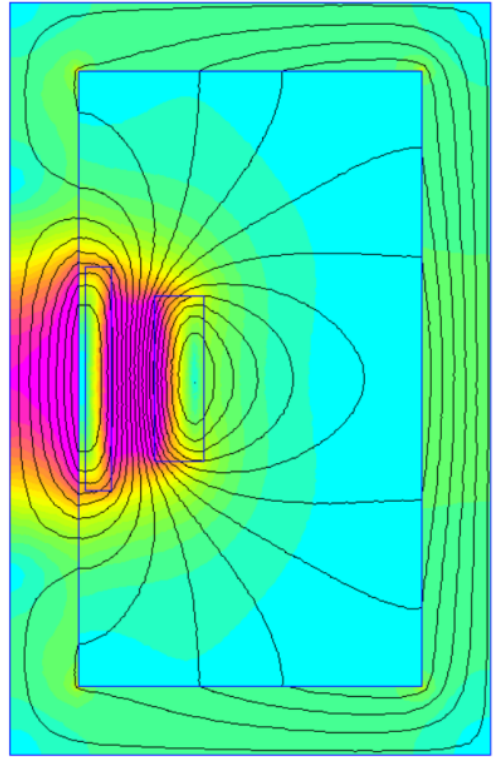

(b)

Figure 4-13. Flux distribution inside the core window obtained by FEM for Decagon MFT; $A$ winding part inside the core window (a), A winding part outside the core window (b)

The leakage inductances are calculated using the total stored magnetic energy feature. Table 4-1 shows the representative values for the leakage inductances of the MFT inside and outside the core window.

Table 4-1. Leakage inductance results of the FEM

\begin{tabular}{|c|c|c|}
\hline $\begin{array}{c}\text { Inside the core window } \\
(\mu \mathrm{H})\end{array}$ & $\begin{array}{c}\text { Outside the core window } \\
(\mu \mathrm{H})\end{array}$ & $\begin{array}{c}\text { Total } \\
(\mu \mathrm{H})\end{array}$ \\
\hline $\mathbf{2 9 . 1 7}$ & $\mathbf{2 5 . 8 0}$ & $\mathbf{2 6 . 7 1}$ \\
\hline
\end{tabular}

To calculate the total inductance, the isolation gap mean-length $\left(M L T_{\text {iso }}\right)$ has been divided into two parts: one part inside the core window, $M L T_{\text {iso, in }}$ and other part outside the core window, $M L T_{\text {iso,out. }}$ The total leakage inductance is calculated as

$$
L_{\text {leakage }}=\left(L_{\text {leak }, \text { in }} M L T_{\text {iso, in }}+L_{\text {leak }, \text { out }} M L T_{\text {iso,out }}\right) /\left(M L T_{\text {iso, in }}+M L T_{\text {iso,out }}\right)
$$

where $L_{\text {leak, in }}$ and $L_{\text {leak,out }}$ are the leakage inductances calculated inside and outside the core window respectively. As mentioned before, for calculation of inductance outside the core window, it is only required to take the outer walls of the core far enough away.

\subsubsection{Core losses}

The suitability for the relatively high operating frequency is the first criterion for the selection of a core for a medium frequency transformer which experiences high frequency voltage harmonic components of the applied square wave from the DAB side (see Figure 2-1a) [8]. Another crucial criterion for the selection of a core for manufacturing a prototype HVDC MFT is a large window size. This ensures an enough insulation clearance for the high DC voltage which is going to be applied to the HV winding.

Nanocrystalline cores can carry more flux which helps the reduction of the weight and size of the prototype. Because these finished cores can be produced from raw sheets of Nanocrystalline bands, there are several producers which can shape products suitable for individual needs. But the raw material is produced only by very few producers and it is expensive, and the cost of post processing is high. Fortunately, Ferrite cores are relatively cheap and available from several commercial suppliers. 
Of course, it is possible to order cores of larger sizes; however, in order to keep the total cost of manufacturing low, it is decided to select the biggest off the shelf product available on the market.

As mentioned earlier, the prototype MFT's power is $50 \mathrm{~kW}$, the voltage ratio is $421.9 / 4500 \mathrm{~V}$, rated transformer currents are 118.5/11.11 $\mathrm{A}$ and the number of turns is 12/128 for the primary and secondary sides respectively. Using (2-30) a core consists of 10 core stacks of Epcos TDK type B67385U 126/91/20 made of the Ferrite N87material is selected (see Figure 4-14). The physical core area is $20 \times 28=560 \mathrm{~mm}^{2}$ and for 10 core stacks including $20 \mathrm{U}$ cores is $5600 \mathrm{~mm}^{2}$ which is suitable for the design. The maximum working flux density of the core will be $0.31 \mathrm{~T}$ which gives 0.8 of saturation flux density of the core (0.39 $\mathrm{T})$ at room temperature [83].

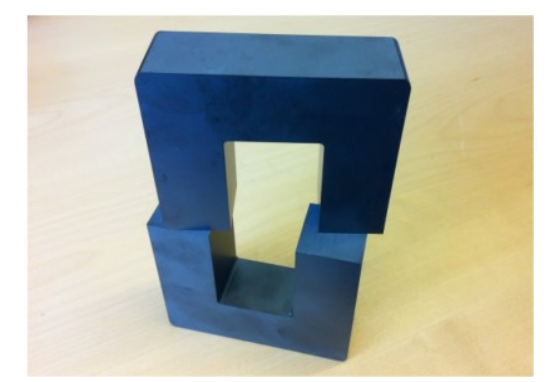

(a)

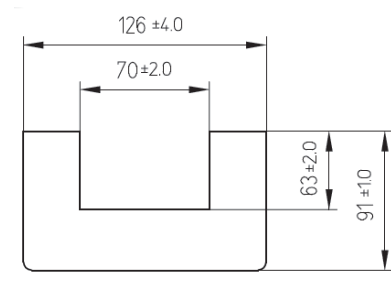

(b)

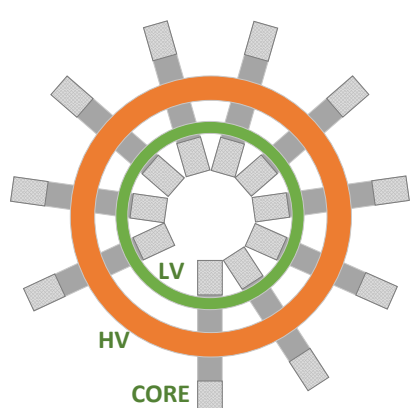

(c)

Figure 4-14. Ferrite core used for the HVDC MFT; Ferrite core shape (a), Core dimensions (b), HVDC MFT cores stacks (c)

In this work, instead of using the empirical equations based on characterization coefficients from the core suppliers earlier mentioned in section 2.4.2.1, the selected core material is characterized by direct core loss measurement and an accurate no-load loss estimation for the prototype transformer are done based the measurements results.

\subsubsection{Winding losses}

In this work, a numerical method based on FEM simulations is presented, which is based on the equivalent permeability and conductivity of the stranded copper conductors. The method is applied to the model of a $5 \mathrm{kHz}$ prototype MFT transformer equipped with Litz wires, and different simulations, with and without the usage of the equivalent model, are performed. Winding losses are calculated at different frequencies. Subsequently, the winding losses of the prototype MFT are measured at different frequencies and compared with the results of simulations. The results of simulations have an acceptable conformity with the presented measurements. Therefore, the proposed method can be used by designers of MFTs as an effective and quick way to easily estimate the winding losses of MFTs where Litz conductors are used.

As shown in Figure 2-1a, the current wave form is similar to a sinusoidal shape and for any simulation or measurement experiment it seems enough to just apply the fundamental element of the medium frequency current wave to estimate the losses in the windings.

The complex permeability and conductivity defined by (2-50) and 2-51) are applied in simulations for the modelling of the Litz wires as a continuum.

The finite element simulations were performed in FEMM, which uses approximate but closed-form expressions for the equivalent conductivity and permeability of regions filled with hexagonally packed round wires, allowing proximity and skin effects to be included with ease in $2 D$ AC field computations. 
The distribution of flux and current densities with equivalent material representation for $5 \mathrm{kHz}$ is shown in Figure 4-15. Solutions were performed over a range of frequencies between 0 and $15 \mathrm{kHz}$. The value of losses and increase of losses in relation to DC losses are illustrated in Table 4-2 showing an increase of losses equal to $21 \%$ at $5 \mathrm{kHz}$, the fundamental frequency, and a value of $189 \%$ at 15 $\mathrm{kHz}$, the third harmonic frequency of the current waveform in the MFT. As it is explained in section 6.1.3, these results are in agreement with the actual measurements on the prototype MFT.

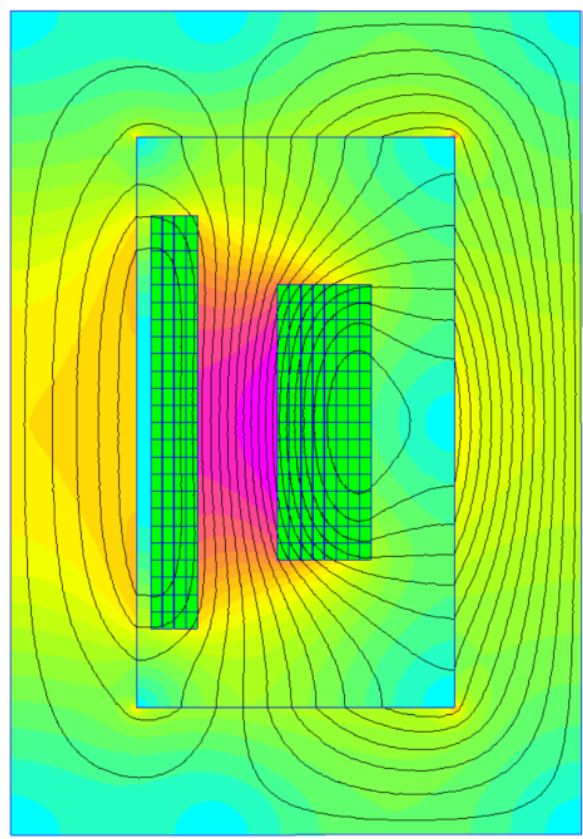

(a)

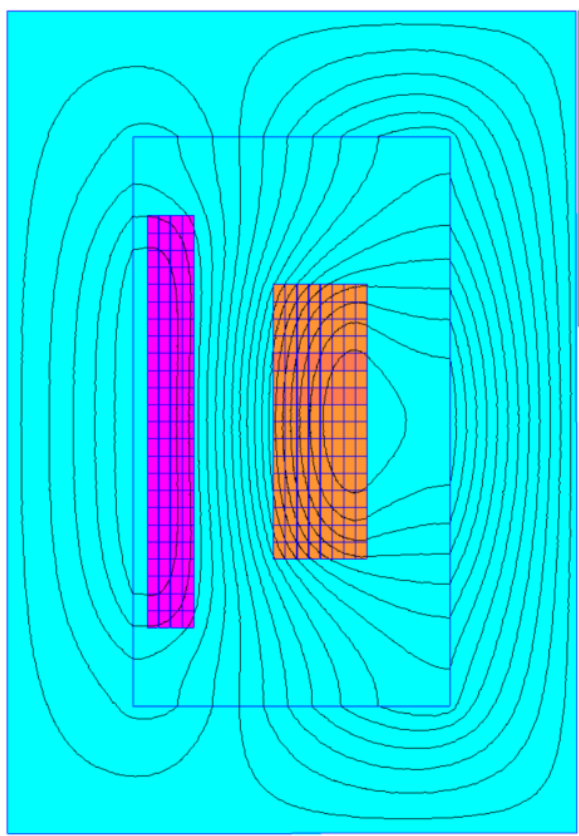

(b)

Figure 4-15. Winding losses analysis of the MFT with equivalent material representation for $5 \mathrm{kHz}$; Flux density distribution (a), Current density distribution (b)

Table 4-2. Results of loss analysis with equivalent material representation method (see section 6.1 .3 for the loss measurement results)

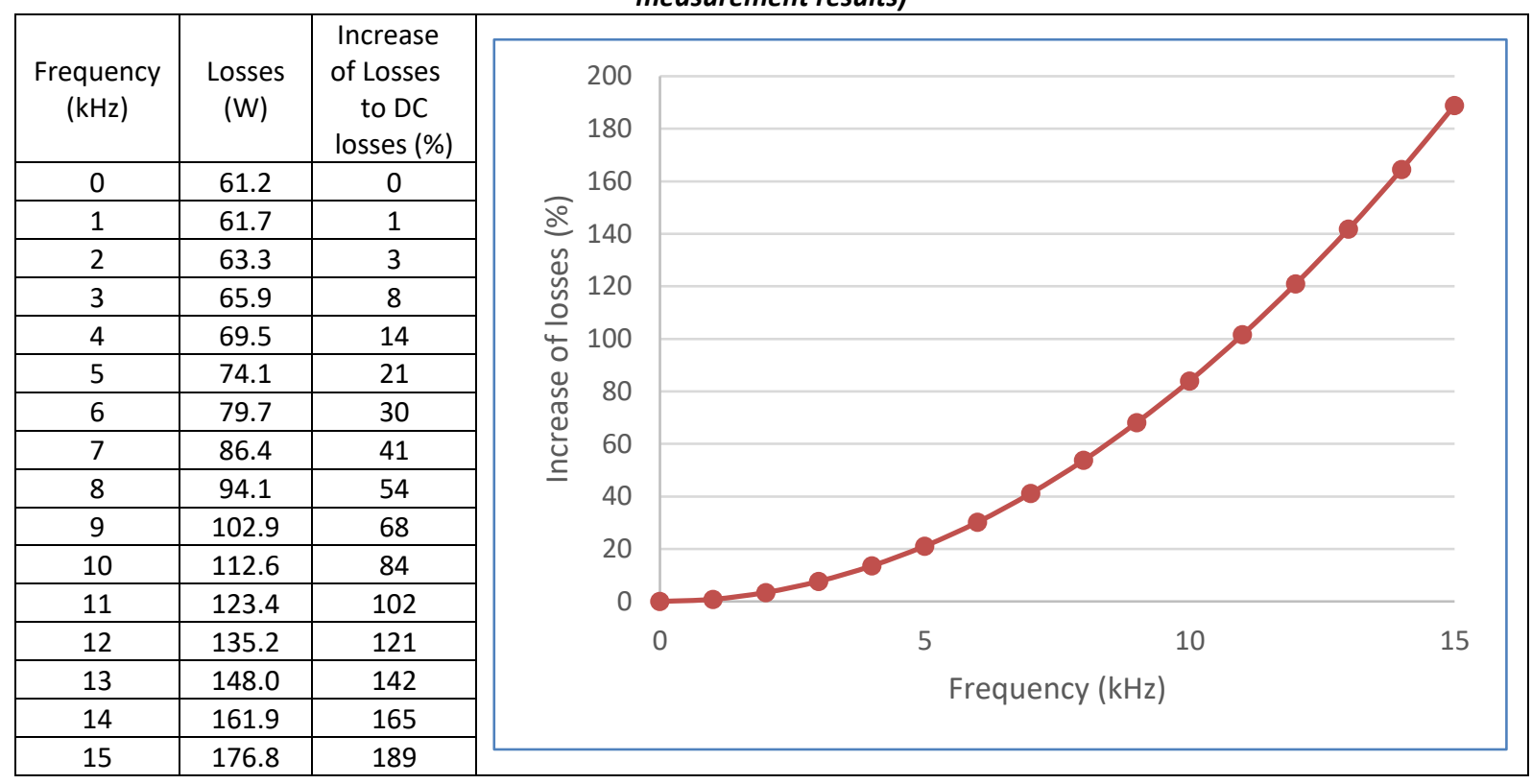

The validity of the method was explored by a comparison of finite element solutions with an equivalent and explicit model of a wound coil. The model with explicit windings needs a fine mesh inside the wires in order to adequately model skin and proximity effects at high frequencies. Since an 
analysis of a problem with all wires modelled with explicit Litz strands is not possible due to the huge amount of required mesh elements at high frequencies, only one wire is modelled explicitly at each time and the total losses that occur inside its copper strands is extracted and compared with the one that is obtained from the equivalent material representation method. Each wire consists of 186 round copper strands wound in alternating layers of 16 and 15 strands in the columns.

The change of the current distribution inside the individual strands of a Litz wire at different frequencies is shown in Figure 4-16. The results of the loss analysis for a Litz wire is shown in Table 4-3 demonstrating that the equivalent material model provides good agreement with the explicit model over the whole frequency range from 0 up to $15 \mathrm{kHz}$.

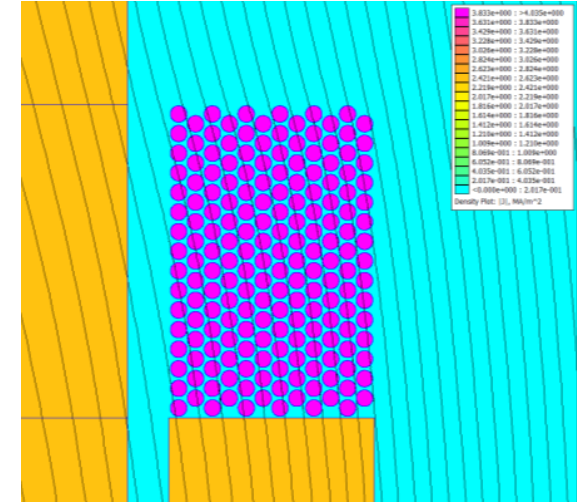

(a) $0 \mathrm{~Hz}(\mathrm{DC})$

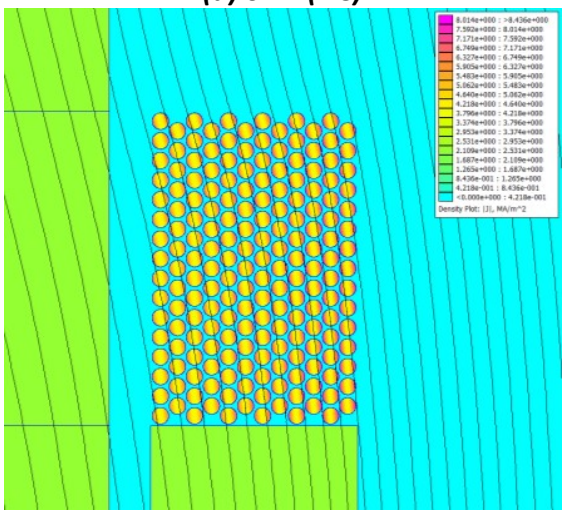

(c) $10 \mathrm{kHz}$

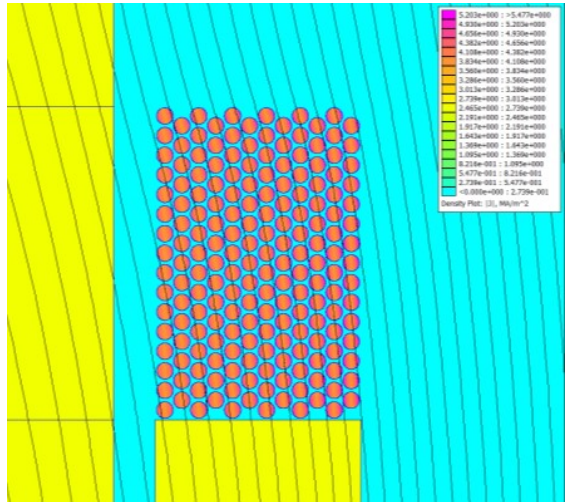

(b) $5 \mathrm{kHz}$

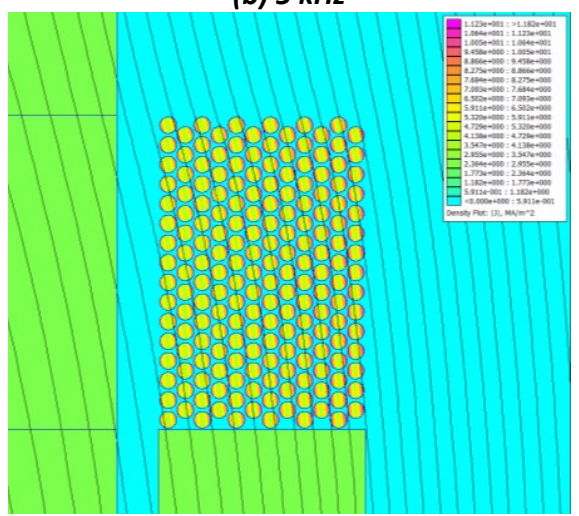

(d) $15 \mathrm{kHz}$

Figure 4-16. Current distribution inside individual strands of a Litz wire at different frequencies

Table 4-3. Comparison of Litz wire losses for explicit and equivalent material models

\begin{tabular}{|c|c|c|c|c|}
\hline Frequency $(\mathrm{kHz})$ & 0 & 5 & 10 & 15 \\
\hline Exact Method & 0.827109 & 0.951974 & 1.32653 & 1.95066 \\
\hline Equivalent Material Representation & 0.827067 & 0.951871 & 1.32624 & 1.95008 \\
\hline
\end{tabular}

Depending on the frequency, the presence of these additional losses will make the AC current losses higher than the equivalent DC current copper losses. Circulating current losses can be a result of having parallel conductors of a different length or exposed to a different electric field which leads to a different voltage drop over two parallel conductors of the same length. For the prototype transformer considered in this study, parallel conductors are axially ordered and there are no circulating current losses in them. However, because of relatively high frequency, eddy current losses are present and will be investigated. 


\section{MFT prototypes manufacturing}

The transformer tank and the active part support structure were made of the extruded thermoplastic acrylic (Plexiglas) which has an excellent electrical insulation property and offers good chemical and thermal resistance to warm transformer oil. An oil circulation and cooling system together with an oil expansion cylinder all from off the shelf materials was connected to the oil tank to feed in the cold oil from bottom and to suck out oil from top. To make a better natural circulation and flow distribution, the oil inlet and outlet pipes were made both in pair and inlets and outlets placed in 90 degrees position. All the piping system were made of oil and heat resistant, electrically insulating plastics (see Figure 5-1).
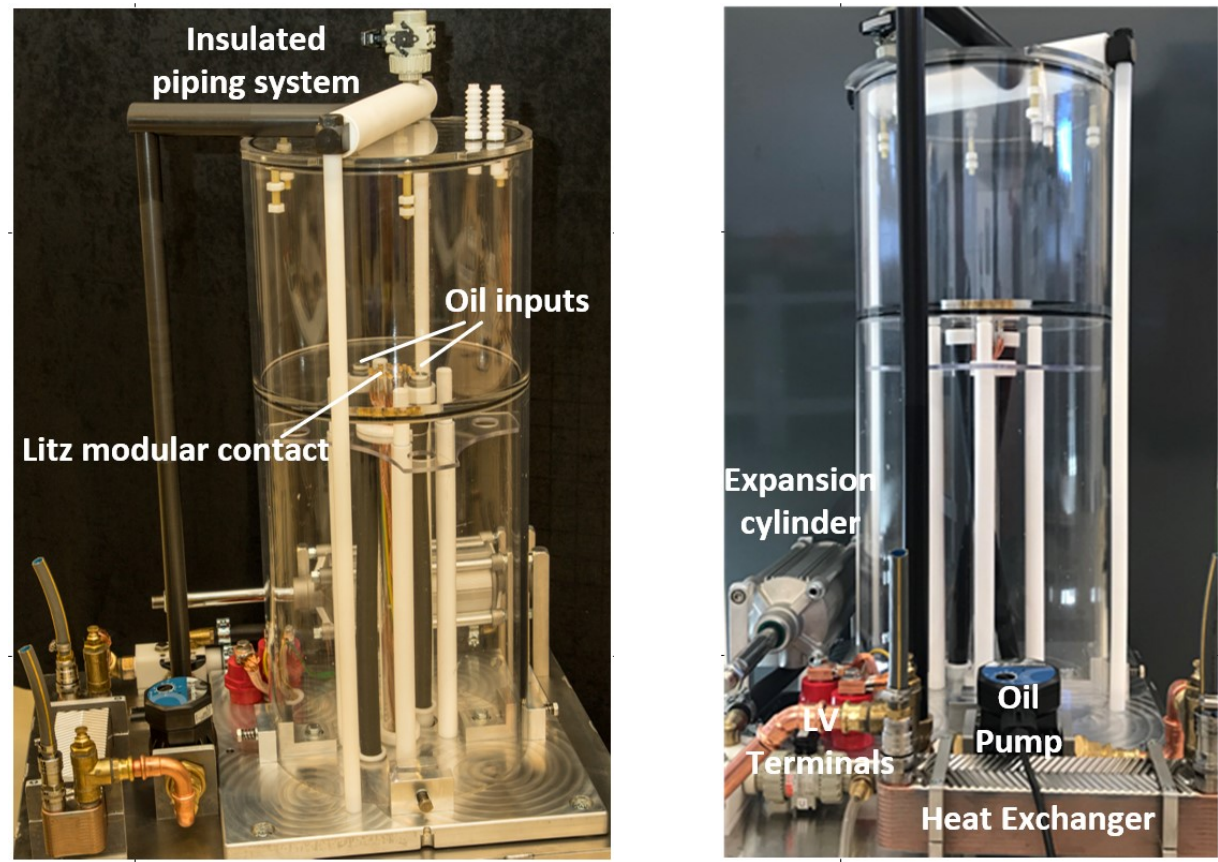

Figure 5-1. Transformer tank and active part mechanical structure

As the tank itself takes the role of the isolation of $125 \mathrm{kV} \mathrm{DC}$ to ground, the only isolation required is between two $5 \mathrm{kV}$ winding terminals which were made of Teflon (see Figure 5-2a). All the cores were grounded using copper strips (see Figure 5-2b). 8+8+1 female banana contacts in the bottom plate of the tank are connected to the two LV terminals outside tank on the bottom plate using 17 Litz wires.

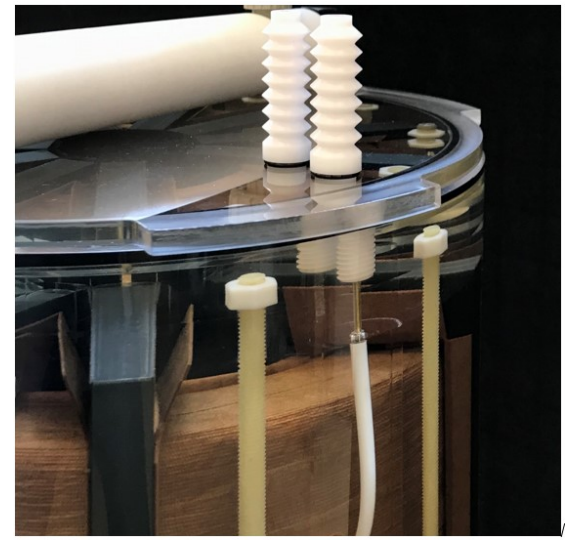

(a)

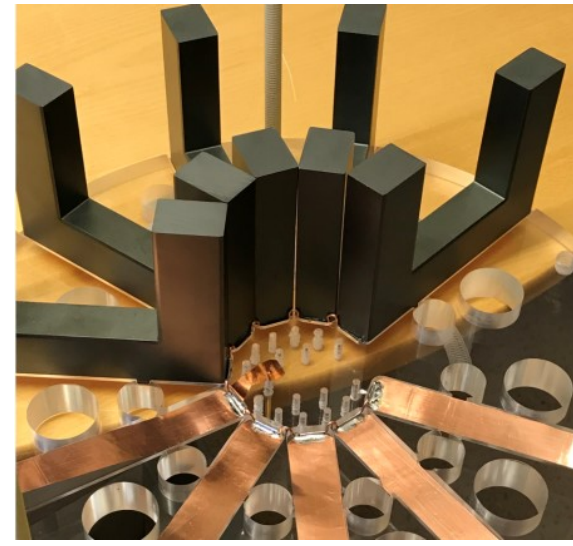

(b)

Figure 5-2. HV bushings and core grounding strips; The HV bushings for isolation of 5 kV AC (a), The grounding copper strips for Ferrite cores (b) 
Each active part includes two round extruded thermoplastic acrylic (Plexiglas) discs which press the cores using epoxy threaded rods. There are enough holes in the discs to let a smooth flow of the oil through the insulated active parts and the core. The active parts have two banana contacts on top and $8+8+1$ banana contacts at the bottom which can sit directly on the female banana contacts at the bottom of the tank. Each Litz wire of 8 has its own banana contact to reduce the extra losses in the external connections to a minimum. The low voltage and high voltage windings of the magnetic prototypes are of layer and disc type respectively as explained in section 4.1. The LV winding has 8 parallel and the HV winding has one rectangular Litz winding (see Figure 5-3a).

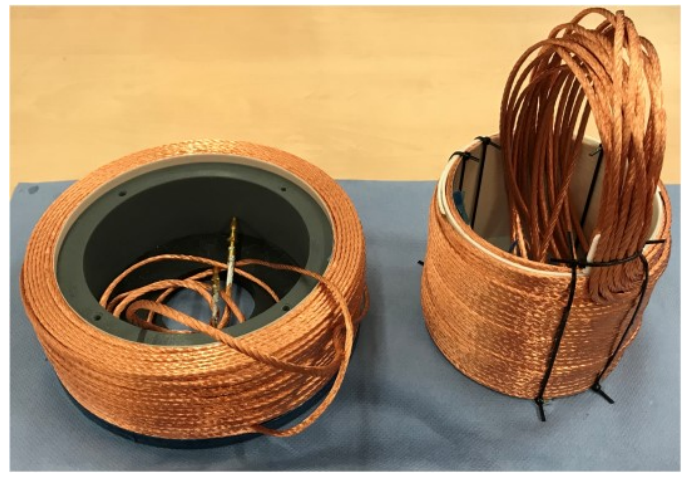

(a)

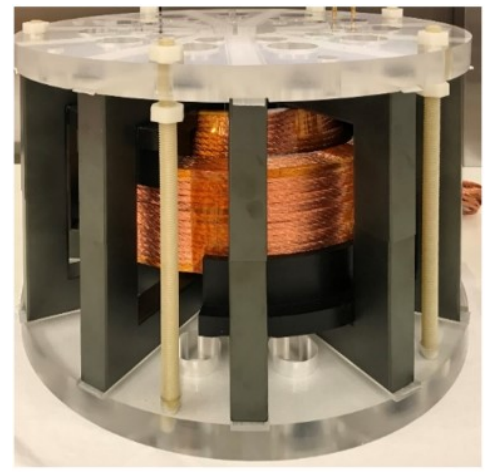

(b)

Figure 5-3. Two windings and magnetic active part module which sits inside the transformer tank; HV and LV windings (a), Magnetic prototype (b)

The dimensions of the built magnetic prototype are presented in Figure 5-4.

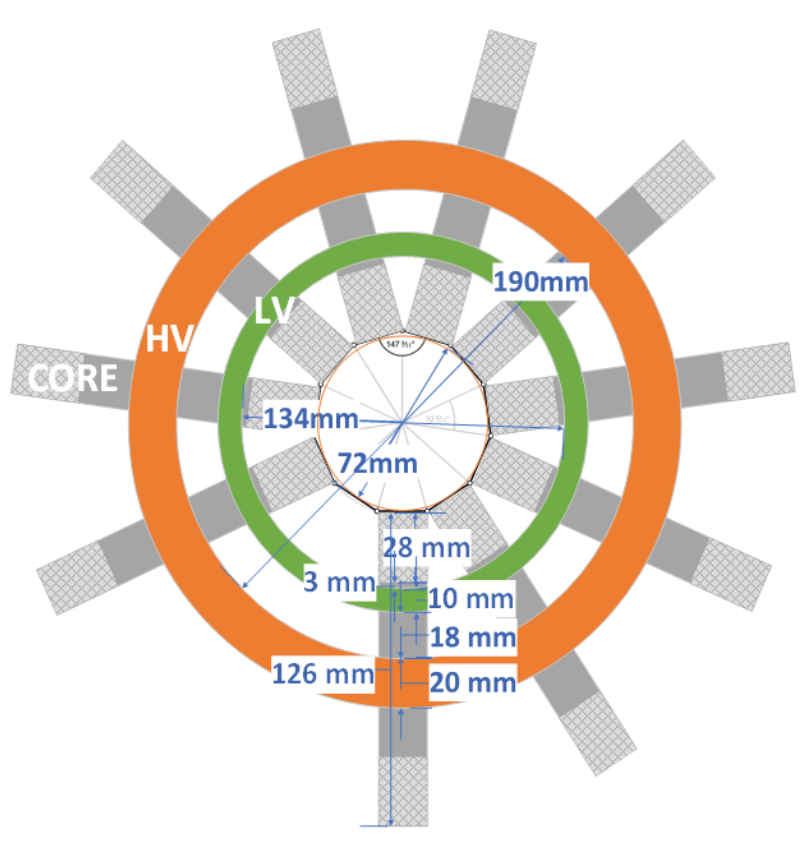

(a)

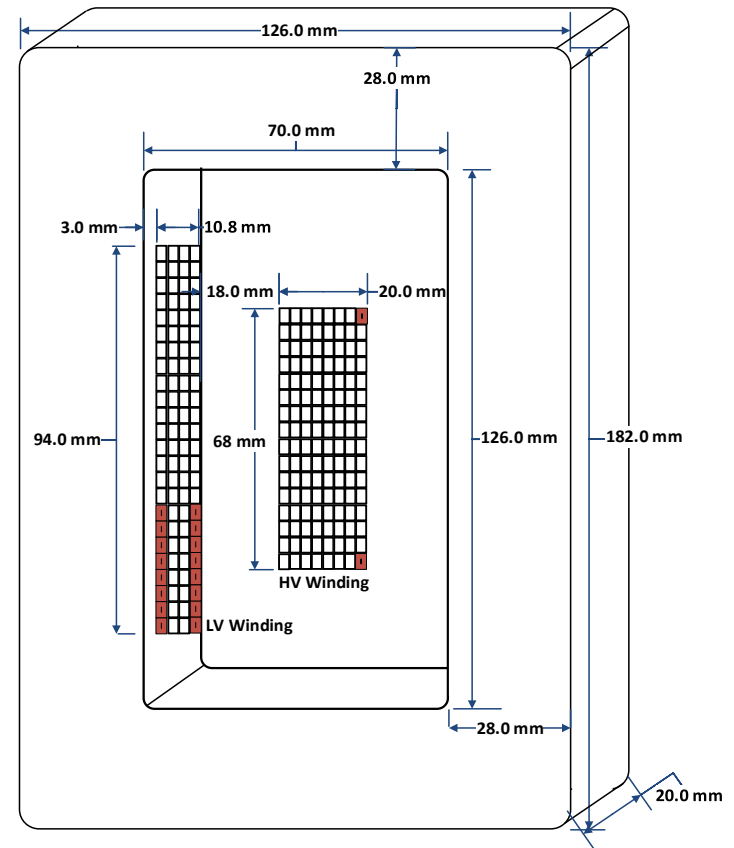

(b)

Figure 5-4. Decagon MFT winding dimensions and their positions inside core window

The LV and HV electrodes of the insulation prototype were made of aluminium cylinders exactly replicating the windings (see Figure 5-5). 


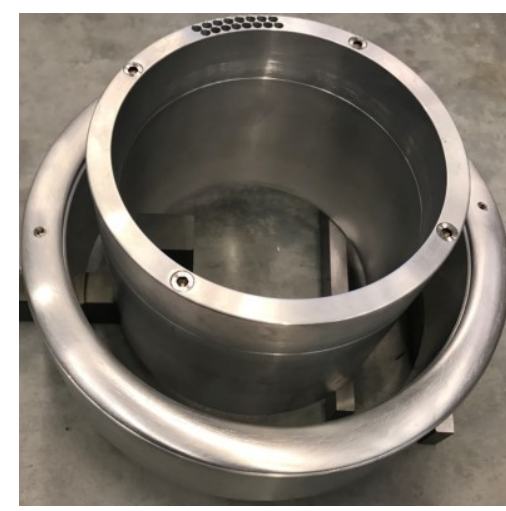

(a)

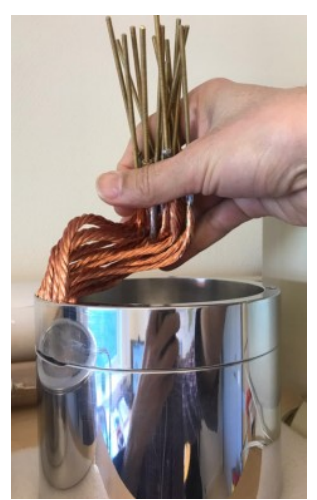

(b)

Figure 5-5. The replica of windings made for insulation prototype; Insulation prototype winding replicas (a), LV electrode with all $8+8$ output Litz wires (b)

Main formed insulation barriers were ordered from a supplier. These parts are made of wood pulp clay mould and dried under special conditions [63] (see Figure 5-6)
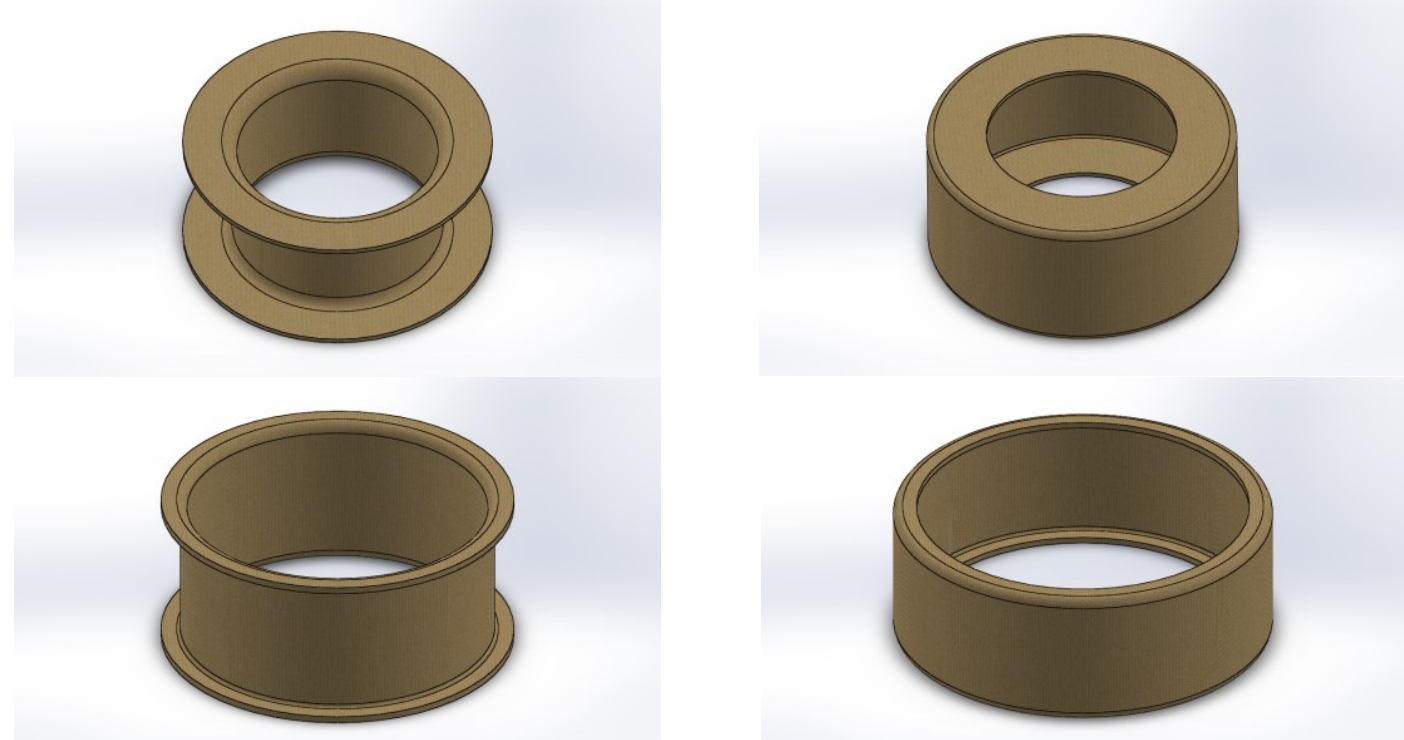

Figure 5-6. Formed insulation parts ordered to a supplier

Figure 5-7a shows the manufactured insulation prototype active part and Figure 5-7b demonstrates a model made by a 3D printer mantled in the active part before ordering the actual insulation parts from the supplier.

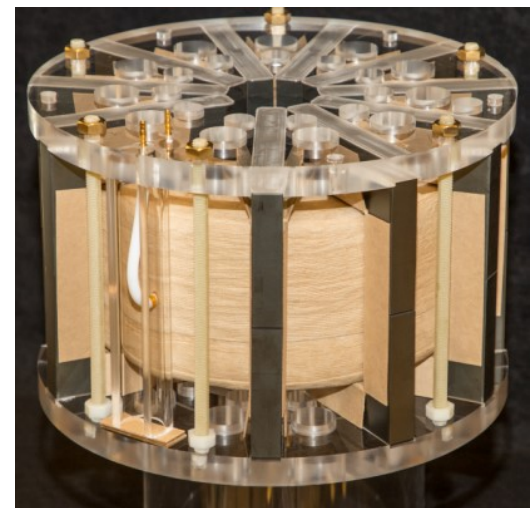

(a)

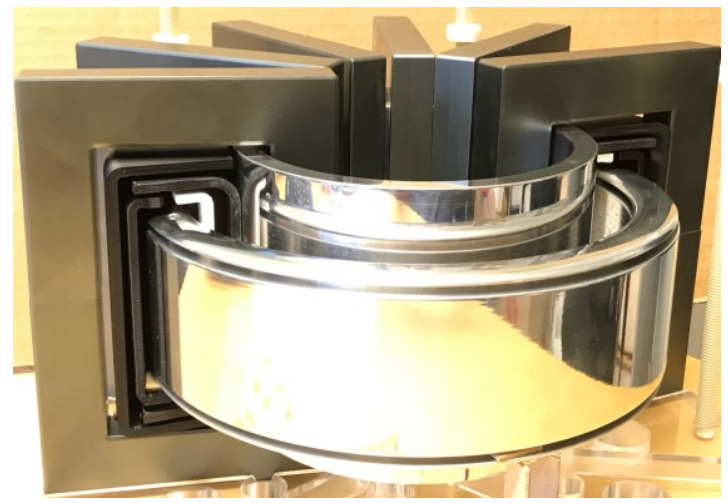

(b)

Figure 5-7. A cut model of insulation parts mantled together with the made coil replicas and cores; The manufactured insulation prototype active part (a), Model 3D printed insulation barriers inside the active part (b) 
The other insulation parts needed are made of cellulose pressboards by the author. $1 \mathrm{~mm}$ pressboards are bent after making them damp on the edges and using dies to keep it in its form during drying (see Figure 5-8).

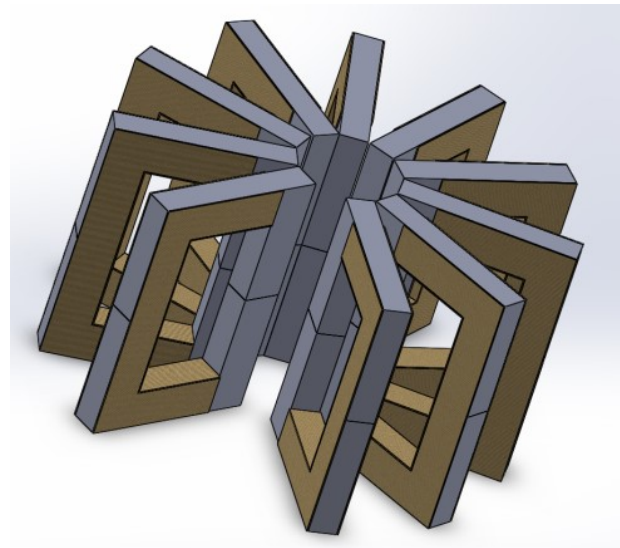

(a)

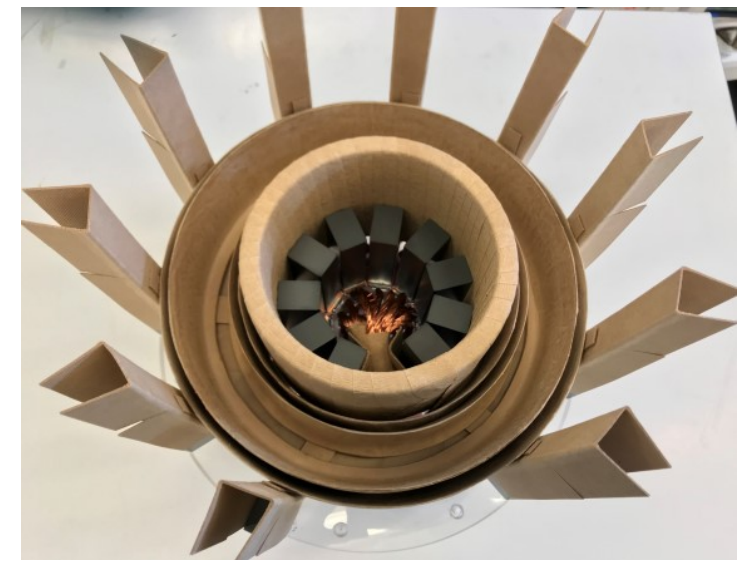

(b)

Figure 5-8. The core barriers during setting up; A model of core protection boards (a), The core protection boards, lower part of formed insulations and the $L V$ winding mantled (b)

Ionized water which has much less impurity of conductive particles compared with tap water was used for damping the dry pressboard before bending (see Figure 5-9b \& c). A Lutron WA-2017 SD water conductivity meter is used to confirm the suitability of the water. $6 \mu \mathrm{S}$ was measured for ionized water compared with $240 \mu \mathrm{S}$ measured for normal the tap water.

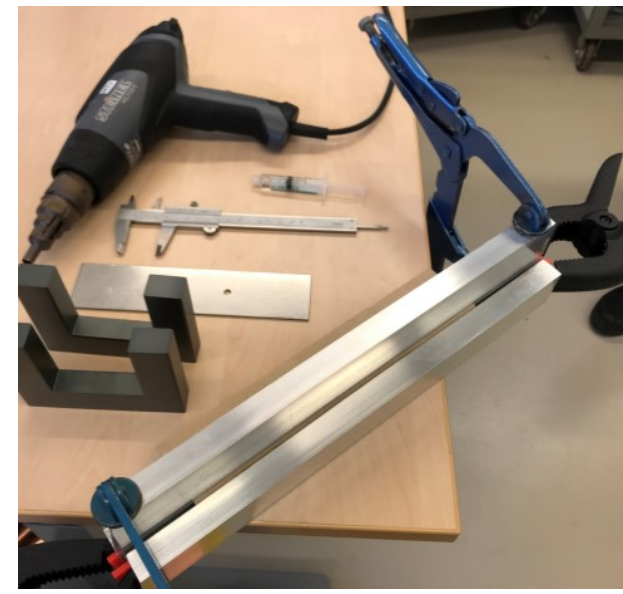

(a)

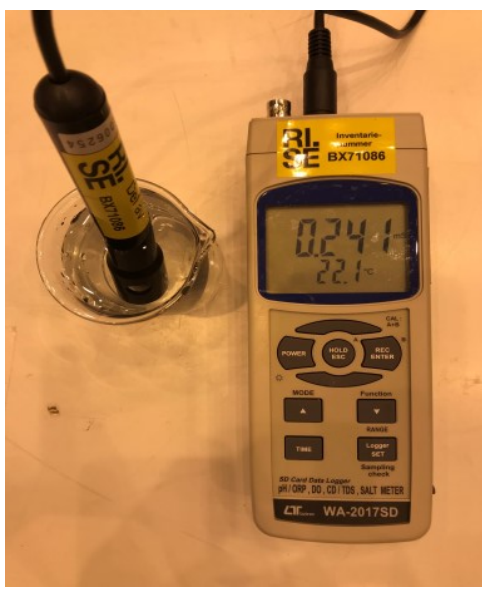

(b)

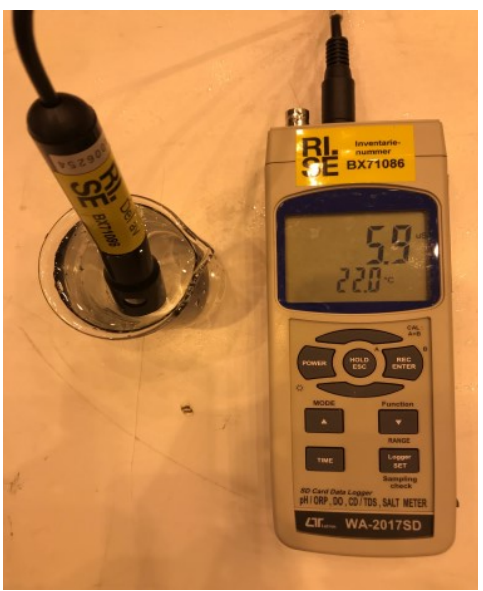

(c)

Figure 5-9. The tools used to form and dry pressboards to formed insulations (a), The conductivity of ionized water (b) compared with the conductivity of tap water (c)

The active part was put in a stainless-steel container, and the container has been placed in $80^{\circ} \mathrm{C}$ for 12 hours and later additionally under full vacuum for 96 hours. Later $80^{\circ} \mathrm{C}$ oil is led into the vacuum chamber trough a needle valve and filled the container from the bottom. After the oil filling the heating was stopped and the system was left under vacuum for 72 hours to cooldown to room temperature.

At the same time the main transformer tank is prepared for receiving the active part. The valve on the top is connected to a $2 \mathrm{~m}$ empty pipe which was connected to a vacuum pump. The valve at the bottom is connected to a barrel of dried and degassed transformer oil at room temperature. 
The active part container is taken out of the vacuum chamber, the humidity content of the oil is checked (see Figure 5-10a), the active part raised and placed in the tank, the tank cover is closed, and vacuum is set to a proper value not over loading the transformer tank mechanical structure. The bottom valve was opened, and the tank was filled with transformer oil from the bottom (see Figure $5-10 b)$. Finally, the transformer is left for 72 hours before testing.

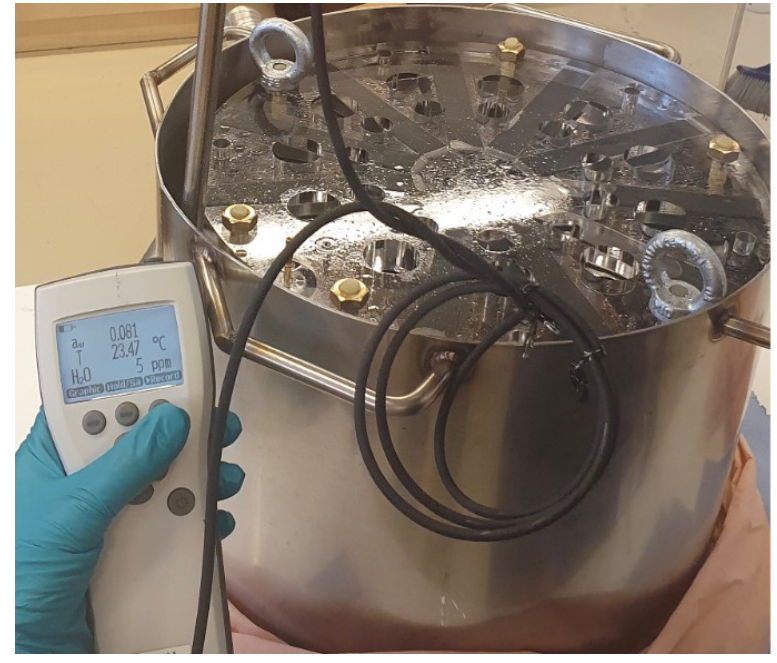

(a)

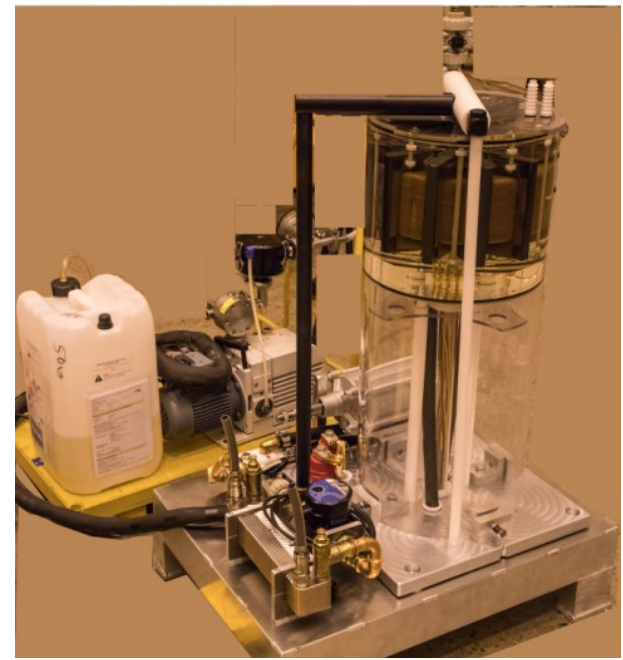

(b)

Figure 5-10. The active part ready to be mantled in the transformer tank; The active part in the oil container after oil impregnation (a), The transformer oil filling setup (b)

The three-stage oil-filling process after putting the active part in the transformer tank is demonstrated in Figure 5-11.

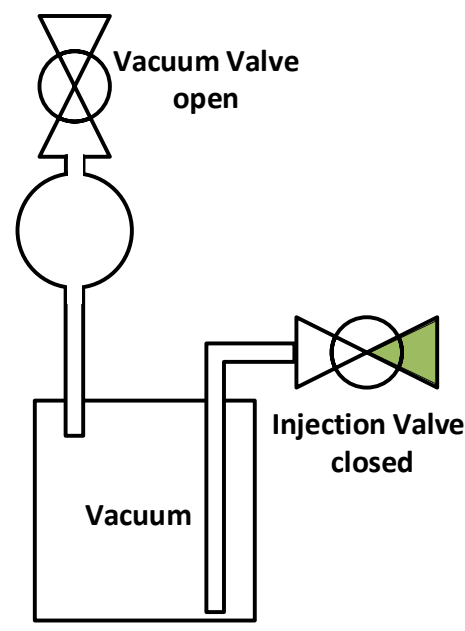

(a)

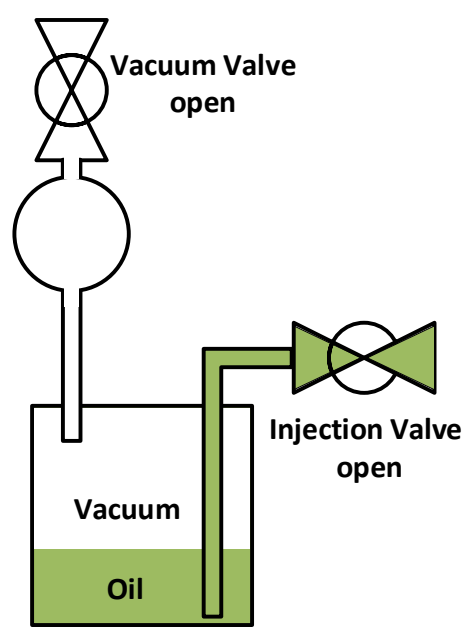

(b)

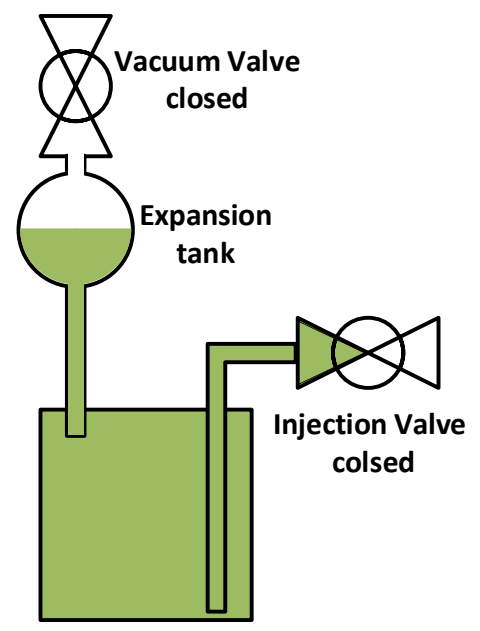

(c)

Figure 5-11. three-stage oil-filling process after putting the active part in the transformer tank; The active part drying and degassing (a), Oil injection under vacuum (b), The Oil filled tank (c) 


\section{Verification tests and analysis}

\subsection{Tests on magnetic prototype}

\subsubsection{Leakage inductance}

Table 6-1 presents the dimensions of constructed prototype transformer which will be used in this section.

Table 6-1. Dimensions of constructed prototype transformers ( $\mathbf{m m}$ )
\begin{tabular}{|l|c|}
\hline Number of core stacks & 10 \\
\hline Core stack width & 20 \\
\hline Core thickness & 28 \\
\hline Core window height & 126 \\
\hline Core window width & 70 \\
\hline & \\
\hline Primary winding number of layers x turns per layer & $4 \times 3$ \\
\hline Secondary winding number of discs x turns per disc & $16 \times 8$ \\
\hline & \\
\hline Primary-Secondary isolation gap mean length (perimeter) & 540 \\
\hline Part of $M L T_{\text {iso }}$ inside the core window (ten 20 mm cores) & 200 \\
\hline Part of $M L T_{\text {iso }}$ outside the core window & 340 \\
\hline
\end{tabular}

To verify the simulation method, inductance measurements have been performed on the Decagon magnetic prototype MFT built in this project. Ferrite cores are used for the design (see Figure 6-1a). The measurement of leakage inductance has been performed using an Agilent E4980A RLC meter. The measurements are performed from both sides. If the measurement is performed from the secondary winding terminals, the primary winding shall be short-circuited (see Figure 6-1b).

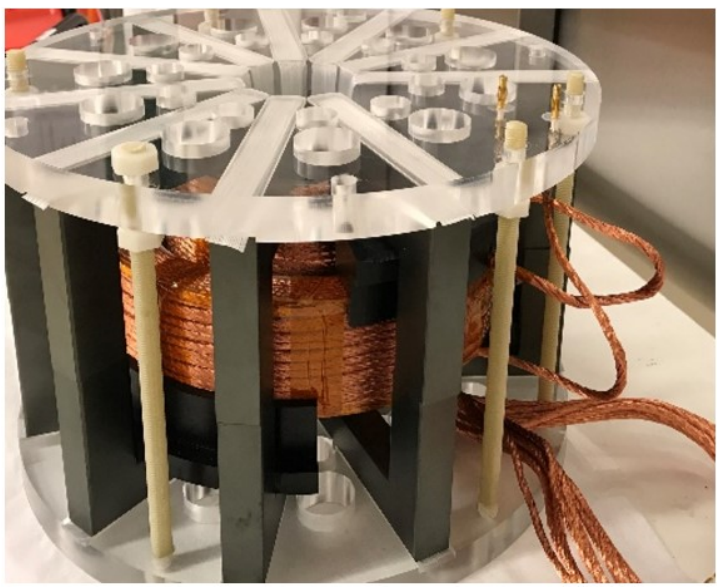

(a)

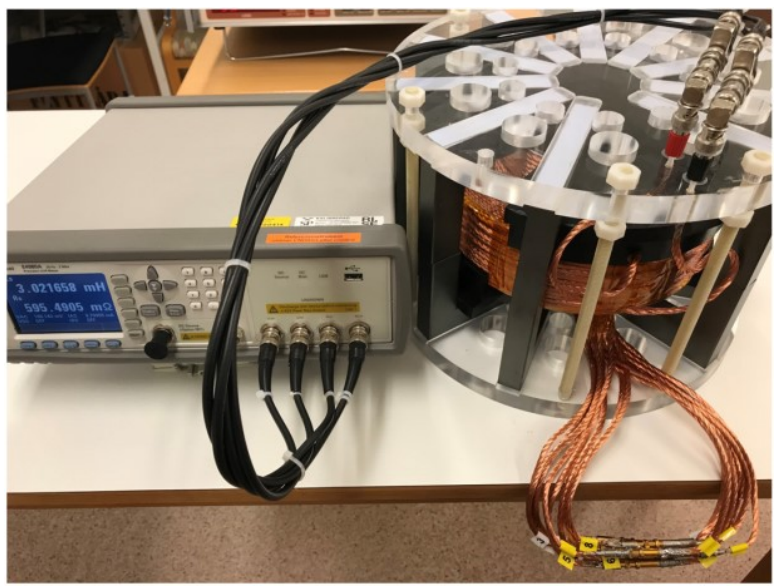

(b)

Figure 6-1. Constructed prototype transformers and leakage inductance measuring test setup; Decagon MFT (a), Leakage inductance measuring circuit (b)

The results of the measurements are presented in Figure 6-2 which indicates a value of $26.47 \mu \mathrm{H}$ at $5 \mathrm{kHz}$. The change of inductance is not significant due to the use of the Litz wire in the windings. It is noticeable that the skin depth of copper at $10 \mathrm{kHz}$ is about $0.73 \mathrm{~mm}$ (with $\sigma=4.74 \mathrm{e}^{7} \mathrm{~S} / \mathrm{m} \mathrm{at} 75^{\circ} \mathrm{C}$ ) while the diameter of each strand of the Litz wire is only $0.2 \mathrm{~mm}$. Therefore, no significant change is observed in the leakage inductance value in the frequency range of $100 \mathrm{~Hz}$ to $10 \mathrm{kHz}$. 


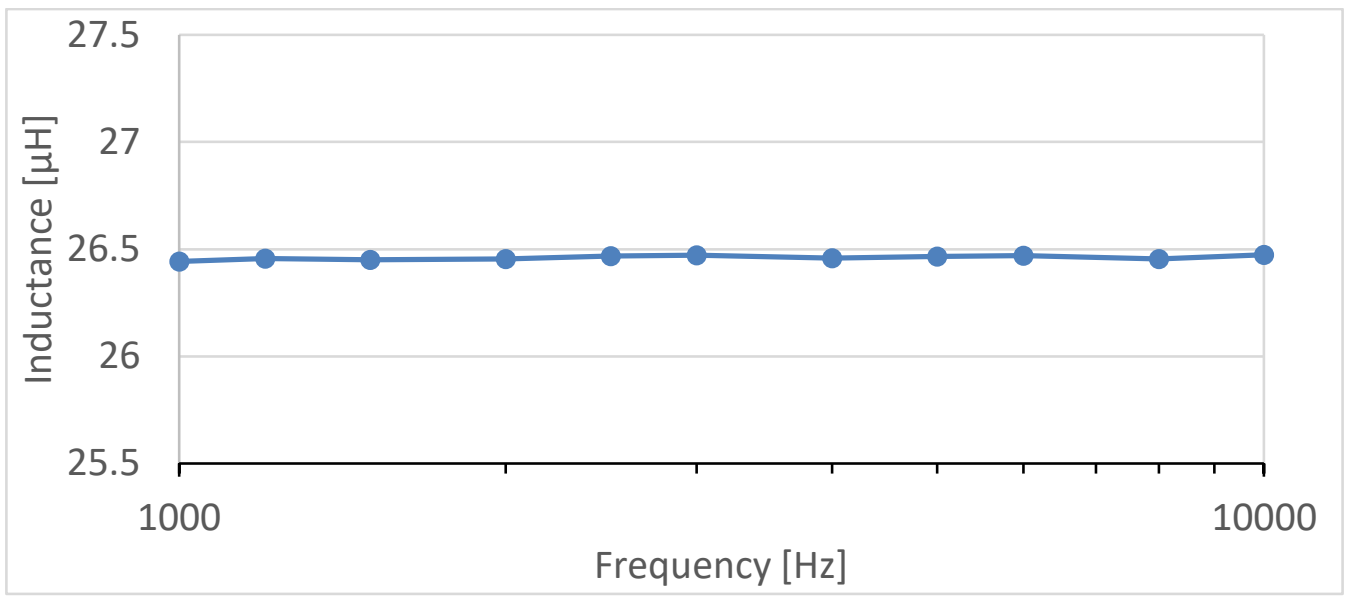

Figure 6-2. Constructed prototype transformers

It can be noticed from Table 4-1 that the leakage inductances computed using the FEM $(26.71 \mu \mathrm{H})$ is in very close agreement to the measurement result.

\subsubsection{Core losses}

Core losses can be measured by two methods; using a power analyser or using a Volt-Ampere method.

\subsubsection{Volt-Ampere method}

The Volt-Ampere method is implemented by a Brockhaus MPG200 measuring unit (see Figure 6-3a) which is used for a series of loss measurements in this work. This method is based on an instantaneous voltage-current sampling and integration method and uses the current signal sampled from the primary side (using an magnetising current from supply side) together with the voltage signal sampled from the secondary side (open circuit voltage which is equal to the magnetizing voltage from the primary side considering the transformation ratio) (see Figure 6-3b).

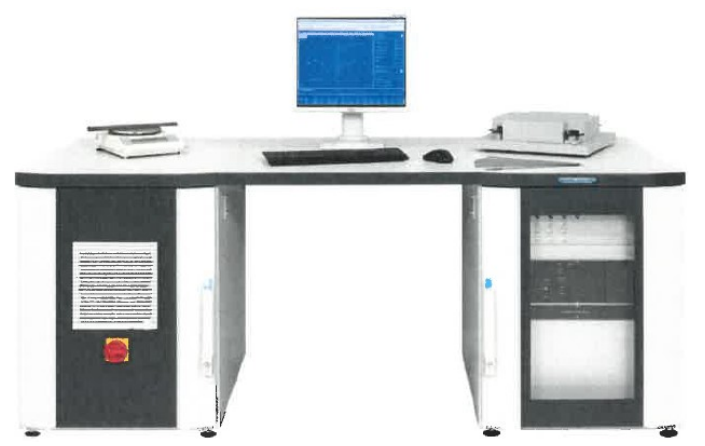

(a)

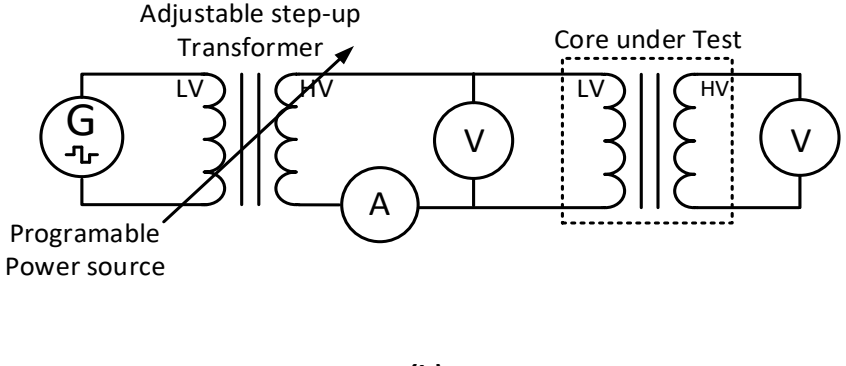

(b)

Figure 6-3. The circuit diagram for measuring core losses; Brockhaus MPG200 core test system (a), Volt-Ampere method circuit diagram (b)

The product of voltage and current samples are integrated over a set of measuring cycles using [109]

$$
P_{i}=\frac{1}{w_{e} \cdot T} \frac{N_{1}}{N_{2}} \int_{0}^{T} v_{L} i_{L} d t
$$

where $w_{e}$ is the mass of the core, $T$ is the time period, $N_{1}$ is the number of the primary winding turns; $N_{2}$ is the number of the secondary winding turns, $v_{L}$ is the voltage on the open secondary side and $i_{L}$ is the current at the primary side.

The tests have been performed applying a precise wave shape and frequency on a type of Nanocrystalline cores and a type of Ferrite core (see Table 6-2). 
Table 6-2. The core samples undergone the measurements

\begin{tabular}{|l|c|c|}
\hline & Nano & Ferrite \\
\hline Core type & Hitachi FINEMET ${ }^{\circledR}$ F3CC Series Cut Core $[84]$ & $93 / 152 / 30 \mathrm{~N} 87[83]$ \\
\hline Weight $[\mathrm{kg}]$ & 2.2625 & $754.7 \times 2$ \\
\hline Mean magnetic length $L_{m}[\mathrm{~mm}]$ & 410.2 & 352.9 \\
\hline Filling factor $k_{c}$ & $693 /(29.6 \times 30)=0.78$ & 1 \\
\hline Density $\left[\mathrm{kg} / \mathrm{m}^{3}\right]$ & 7300 & 4850 \\
\hline Magnetic cross section $A c\left[\mathrm{~mm}^{2}\right]$ & $30 \times 29.9 \times 0.78=699.7$ & 840 \\
\hline Operating temp $\left[{ }^{\circ} \mathrm{C}\right]$ & $20-155$ & $25-100$ \\
\hline
\end{tabular}

Demagnetization before each measurement has been performed. Measurements has been performed in room temperature. Four windings are wound to be used for supplying the magnetizing current and to pick up the voltage on the two core limbs. The limit of $100 \mathrm{~V}$ and $52 \mathrm{~A}$ from the unit has dictated the selected number of turns and type of connections of the windings (series or parallel) (see Figure 6-4).

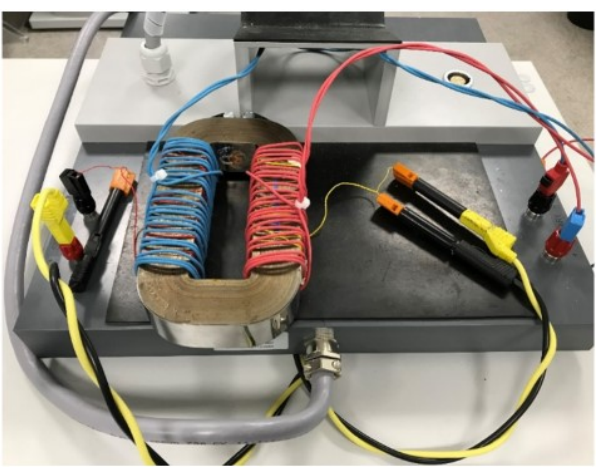

(a)

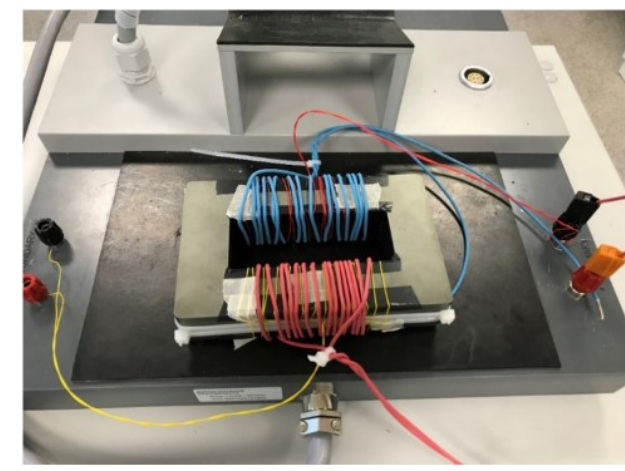

(b)

Figure 6-4. Voltage pickup and current supplying coils connected to the measuring circuits; Nanocrystalline core (a), Ferrite core (b)

A sample recorded current, voltage and $B / H$ curve at $5 \mathrm{kHz}$ applying sinusoidal and square wave shapes are presented in Figure 6-5. The test results are summarized in Table 6-3. It is noticeable that at room temperature, the losses are not dependent of the wave shape change from sinusoidal to square wave shape at $5 \mathrm{kHz}$ if the flux density is kept under $0.3 \mathrm{~T}$. This becomes clearer comparing the recorded $\mathrm{B} / \mathrm{H}$ curves at different excitation voltage shapes.

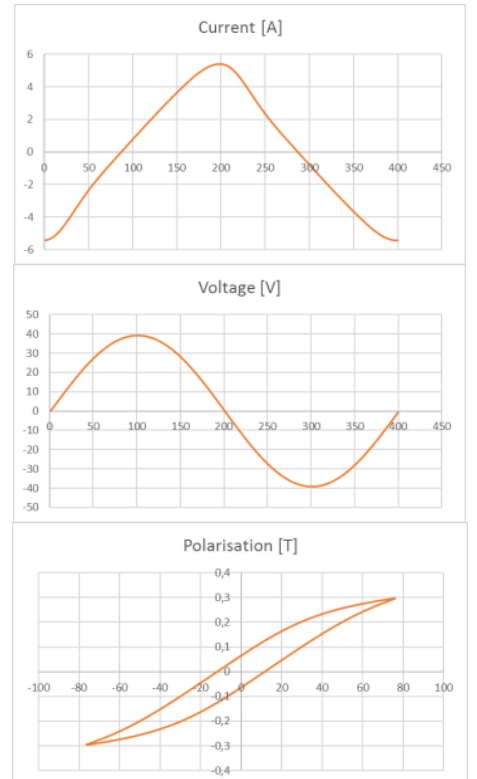

(a) Sinus $5 \mathrm{kHz}, 0.3 \mathrm{~T}$

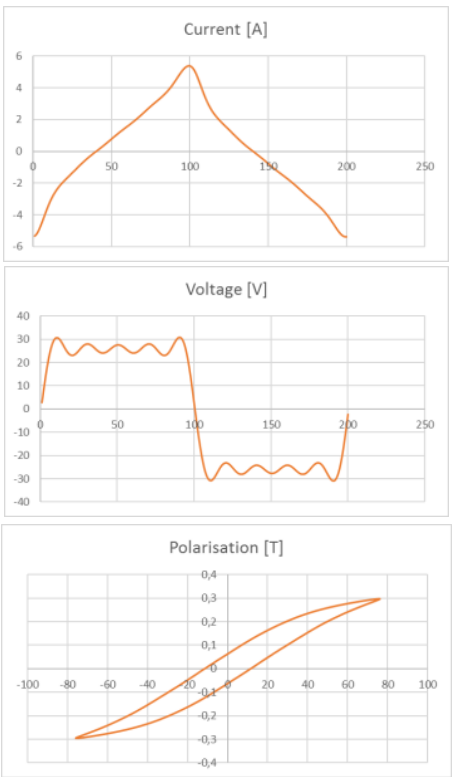

(b) Square $5 \mathrm{kHz}, 0.3 \mathrm{~T}$

Figure 6-5. The recorded current, voltage and $B / H$ curves applying different wave shapes 
Table 6-3. Summary of core loss measurements on two high frequency core samples

\begin{tabular}{|c|c|c|c|c|c|c|c|c|}
\hline \multirow{3}{*}{$\begin{array}{c}\begin{array}{c}\text { Ps } \\
{[\mathrm{W} / \mathrm{kg}]}\end{array} \\
{[\mathrm{T}]} \\
\mathrm{f}[\mathrm{kHz}]\end{array}$} & \multicolumn{4}{|c|}{ Nanocrystalline } & \multicolumn{4}{|c|}{ Ferrite } \\
\hline & \multicolumn{2}{|c|}{$\begin{array}{c}0.1 \\
\text { (Data sheet) }\end{array}$} & \multicolumn{2}{|c|}{$\begin{array}{c}1.0 \\
(=1.23 \times 0.8) \\
\end{array}$} & \multicolumn{2}{|c|}{$\begin{array}{c}0.3 \\
\left(=0.38 @ 100^{\circ} \mathrm{C} \times 0.8\right) \\
\end{array}$} & \multicolumn{2}{|c|}{$\begin{array}{c}0.4 \\
\left(=0.49 @ 25^{\circ} \mathrm{C} \times 0.8\right)\end{array}$} \\
\hline & Square & Sinus & Square & Sinus & Square & Sinus & Square & Sinus \\
\hline 1 & 0.0108 & 0.0121 & 1.333 & 1.3096 & 2.2679 & 2.1599 & 4.2998 & 4.2147 \\
\hline 2 & 0.0227 & 0.0283 & 2.937 & 3.2474 & 4.3549 & 4.4404 & 8.1522 & 8.6792 \\
\hline 4 & 0.0561 & 0.0724 & $\begin{array}{c}8.0898 \\
(0.975 T)\end{array}$ & 8.9581 & 9.0285 & 9.3601 & $\begin{array}{c}17.4141 \\
(0.39 \mathrm{~T})\end{array}$ & 17.8390 \\
\hline 5 & 0.0788 & 0.0909 & $\begin{array}{l}10.291 \\
(0.96 \mathrm{~T}) \\
\end{array}$ & 12.292 & 11.6345 & 11.6518 & $\begin{array}{c}21.4151 \\
(0.38 \mathrm{~T})\end{array}$ & 22.6056 \\
\hline 8 & $\begin{array}{c}0.159 \\
(8.3 \mathrm{kHz}, 0.093 \mathrm{~T})\end{array}$ & 0.2013 & $\begin{array}{c}25.184 \\
(8.3 \mathrm{kHz})\end{array}$ & 24.773 & $\begin{array}{l}20.4339 \\
(8.3 \mathrm{kHz})\end{array}$ & 19.6096 & $\begin{array}{l}40.8608 \\
(8.3 \mathrm{kHz})\end{array}$ & 37.9127 \\
\hline 10 & $\begin{array}{c}0.181 \\
(9.1 \mathrm{kHz} .0 .093 \mathrm{~T})\end{array}$ & $\begin{array}{c}0.261 \\
(9.5 \mathrm{kHz})\end{array}$ & 33,383 & $\begin{array}{c}31,025 \\
(9.5 \mathrm{kHz})\end{array}$ & 25.0809 & 25.2984 & 48.5436 & 49.0182 \\
\hline
\end{tabular}

\section{HVDC MFT core losses}

First to verify the calculation, the measurement procedure is applied to recalculate the already measured core losses of the previously manufactured dry type planar Ferrite design MFT [8]. The total volume of the cores is $0.0030576 \mathrm{~m}^{3}$ and their total weight $14.83 \mathrm{~kg}$. Applying the same loss density of $11.65 \mathrm{~W} / \mathrm{kg}$ (from Table 6-3), the total core losses will be 172.76 which is comparable with the measured value of $171.4 \mathrm{~W}$ at room temperature and the flux density of $0.32 \mathrm{~T}$.

The measurements performed on the Ferrite cores used for HVDC MFT prototype at $0.3 \mathrm{~T}$ demonstrates that for each core the loss at $5 \mathrm{kHz}$ square wave excitation is about $11.65 \mathrm{~W} / \mathrm{kg}$ (see Table 6-3). The density of core is $4870 \mathrm{~kg} / \mathrm{m}^{3}$ according to its datasheet. Consequently, the measured losses can be presented as $56.7 \mathrm{~kW} / \mathrm{m}^{3}$. The total volume of the cores of the HVDC MFT is $0.1386 \times 20$ or $2772000 \mathrm{~mm}^{3}$ and accordingly the total weight of the cores will be $13.50 \mathrm{~kg}$. Therefore, the total core losses for the transformer at $0.3 \mathrm{~T}$ will be approximately $157.1 \mathrm{~W}$. Based on the cores technical data, at a working temperature $80^{\circ} \mathrm{C}$ the core losses will be reduced by $33 \%$ for the prototype and will reach $121 \mathrm{~W}$ [83].

\subsubsection{Direct power measurement method}

To introduce a core loss measuring setup with the off-the-shelf equipment instead of a very expensive and specialized equipment available at $A B B$, a setup is prepared. An audio amplifier is used to amplify the signals generated by a signal generator to the desired voltage level and the core loss measurement has been performed at supply terminals by a precision Power analyser. A temperature chamber is used to bring the core to the desired temperature (see Figure 6-6). 


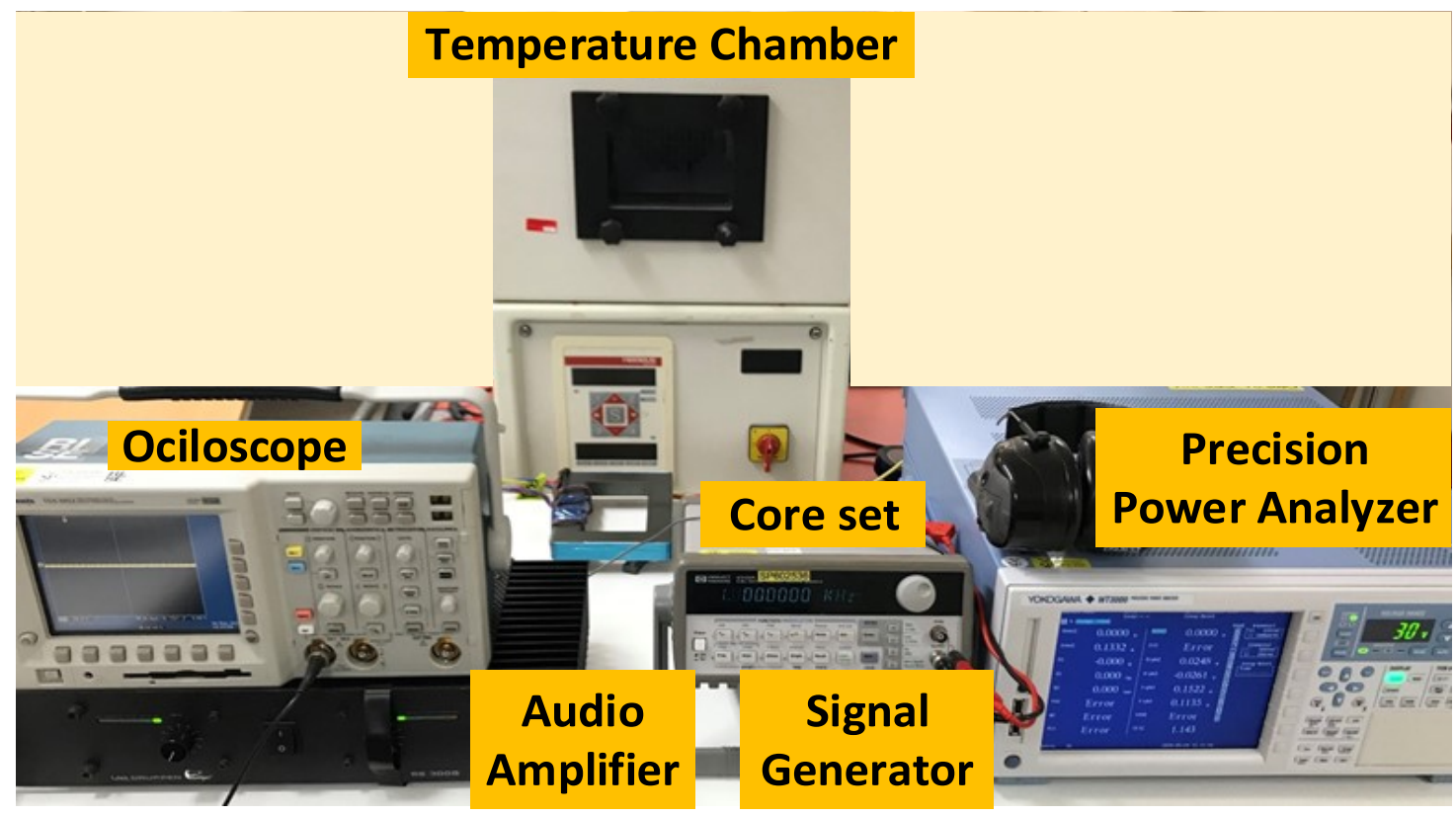

Figure 6-6. Core loss measuring test setup

The second series of measurements are performed using a power analyser which is designed to handle the very low phase angles between the voltage and current. A Yokogawa WT3000, precision power analyser is used for the direct loss measurement. It is obvious that the voltage-drop in the supply winding due to magnetising current is also measured. As a result, the measured loss will be slightly higher than the real loss. To make a correction a 4 turns coil was wound to pick up the supplied voltage to the primary while the supplied current is measured from the main winding. Table 6-4 demonstrates the calculations made to determine the voltage that should be set on a 4-turn winding around the core during a one core set loss measurement.

Table 6-4. Preliminary calculations to perform direct core loss measurements
\begin{tabular}{|l|c|}
\hline Core type & B67385U \\
& $126 / 91 / 20$ \\
\hline$A_{c}$ Core cross section $\left(\mathrm{mm}^{2}\right)$ & 560 \\
\hline$K_{c}$ Filling factor & 1 \\
\hline Weight of one U core (kg) & 0.675 \\
\hline Volume of one U core (m $\left.{ }^{3}\right)$ & 0.0001386 \\
\hline Total weight of cores (kg) & 13.500 \\
\hline Total volume of cores (m3) & 0.002772 \\
\hline Flux density @421.9 V \& 5kHz square (T) & 0.32 \\
\hline Required test Square Voltage @0.32 T \& 5 kHz (V) & 421.90 \\
\hline Applied to 4 turns one core Square Voltage @0.32 T\& 5kHz (V) & 14.06 \\
\hline Required test Sinusoidal Voltage @0.32 T \& 5 kHz(V) & 468.86 \\
\hline Applied to 4 turns one core Sinusoidal Voltage @0.32 T \& 5kHz (V) & 15.63 \\
\hline
\end{tabular}

The required voltage to achieve the rated flux density is calculated for both square and sinusoidal waves using (2-30) and (2-31) respectively. The required voltage to reach the same flux density for the sine wave feeding is higher than the voltage for the square wave. Table 6-5 shows the result of measurements for the sinusoidal- and square- wave-shapes at nominal flux densities of the core in the frequency range of 1 to $10 \mathrm{kHz}$ at room temperature. The results show that the losses at sine wave is slightly higher than for the square wave case. 
Table 6-5. Ferrite N87 core loss measurements results

\begin{tabular}{|c|c|c|c|c|c|c|c|c|c|c|c|}
\hline Frequency $(\mathrm{kHz})$ & & 1 & 2 & 3 & 4 & 5 & 6 & 7 & 8 & 9 & 10 \\
\hline \multicolumn{12}{|l|}{ Square } \\
\hline 4 turns supply & Nom. Volt. & 2.8 & 5.6 & 8.4 & 11.2 & 14.1 & 16.9 & 19.7 & 22.5 & 25.3 & 28.1 \\
\hline Voltage from Aux & App. Volt. & 2.8 & 5.6 & 8.4 & 11.3 & 14.1 & 16.9 & 19.7 & 22.5 & 25.3 & 28.1 \\
\hline \multirow[t]{2}{*}{ One UU core } & Meas. Curr. & 4.4 & 4.4 & 4.4 & 4.4 & 4.5 & 4.5 & 4.5 & 4.5 & 4. 6 & 4.57 \\
\hline & Meas. Loss & 2.9 & 5.9 & 8.9 & 11.9 & 15.0 & 18.0 & 21.0 & 24.5 & 27.3 & 30.6 \\
\hline Ten set of $\frac{\bigcap}{U}$ cores & Total Loss & 29.0 & 59.0 & 89.1 & 119.3 & 149.6 & 179.7 & 210.5 & 244.6 & 273.5 & 306.0 \\
\hline \multicolumn{12}{|l|}{ Sinusoidal } \\
\hline 4 turns supply & Nom. Volt. & 3.1 & 6.2 & 9.4 & 12.5 & 15.6 & 18.7 & 21.9 & 25.0 & 28.1 & 31.3 \\
\hline Voltage from Aux & App. Volt. & 3.1 & 6.2 & 9.4 & 12.5 & 15.6 & 18.7 & 21.9 & 25.0 & 28.1 & 31.3 \\
\hline \multirow[t]{2}{*}{ One UU core } & Meas. Curr. & 5.5 & 5.5 & 5.5 & 5.5 & 5.5 & 5.5 & 5.5 & 5.4 & 5.48 & 5.45 \\
\hline & Meas. Loss & 3.0 & 5.9 & 8.8 & 11.9 & 15.0 & 18.1 & 21.5 & 25.0 & 28.4 & 32.1 \\
\hline Ten set of $\frac{\|}{U}$ cores & Total Loss & 29.6 & 58.4 & 88.2 & 118.6 & 150.0 & 181.5 & 214.9 & 250.3 & 284.4 & 320.6 \\
\hline
\end{tabular}

\subsubsection{Winding losses}

To verify the calculation method, measurements have been performed on the built prototype Decagon MFT (see Figure 6-1a). Table 6-6 presents the dimensions data of the designed prototype.

Table 6-6. Dimensions data of the Ferrite prototype

\begin{tabular}{|l|c|}
\hline & Sizes in (mm) \\
\hline \hline Primary winding & 12 \\
\hline Number of turns (4 layers) & 10 \\
\hline Thickness & 10.8 \\
\hline Thickness (built prototype) & 30.4 \\
\hline Wire height (8 parallel conductors) & 91.2 \\
\hline Magnetic height & 94 \\
\hline Magnetic height (built prototype) & 121.6 \\
\hline Physical height & \\
\hline & \\
\hline Secondary winding & 128 \\
\hline Number of turns (16 discs) & 20 \\
\hline Thickness & 3.8 \\
\hline Wire height & 60.8 \\
\hline Magnetic height & 68 \\
\hline Magnetic height (built prototype) & 60.8 \\
\hline Physical height & \\
\hline
\end{tabular}

\subsubsection{DC resistance}

The measured DC resistance of one strand with the length of $14450 \times 1.04 \mathrm{~mm}(14450 \mathrm{~mm}$ is the conductor length) is $8.32 \Omega$, which is measured using a precision HP 3458 multimeter at $20^{\circ} \mathrm{C}$. The resistivity of copper $\rho$ at $20^{\circ} \mathrm{C}$, is calculated as $1.74 \mathrm{e}-8 \Omega$.m which is comparable with the values given by manufacturers. In addition, the resistance of the complete conductor with the length of $14450 \mathrm{~mm}$ is measured applying $1 \mathrm{~A}$ which gives the resistance of the conductor of $3.2 \mathrm{~m} \Omega / \mathrm{m}$.

The resistances of the finished windings are also measured. The cores' magnetizing circuits were open during the resistance measurement (see Figure 6-7. This omits the possibility of measuring the core magnetizing current in addition to the resistive current, the current stabilizes very quickly, and the measurement time will be reduced dramatically. 


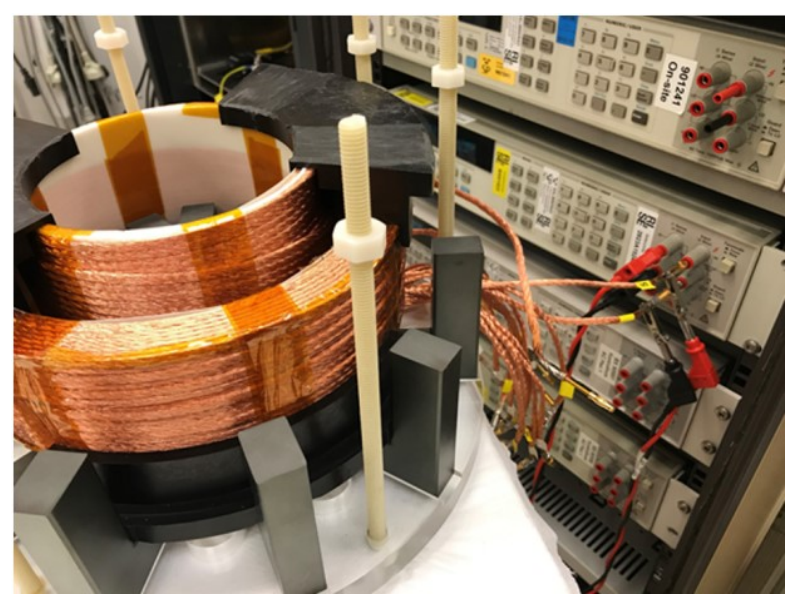

Figure 6-7. DC resistance measuring setup

The measured and calculated DC resistances are presented in Table 6-7.

Table 6-7. Comparison of calculated and measured DC resistances at $20^{\circ} \mathrm{C}$

\begin{tabular}{|l|c|c|}
\hline Measured from & Secondary & Primary \\
\hline Calculated & 274.262 & 2.322 \\
\hline Measured & 276.36 & 2.402 \\
\hline
\end{tabular}

The $R I^{2}$ losses when a DC current is applied is calculated according to Table 6-8.

Table 6-8. Calculated and measured resistances and calculated $R^{2}$ losses

\begin{tabular}{|l|c|c|}
\hline & Secondary & Primary \\
\hline Calculated $\mathrm{R}(\mathrm{m} \Omega)$ & 274.262 & 2.322 \\
\hline Rated r.m.s current (A) & 11.11 & 118.5 \\
\hline Calculated DC, $\left.R\right|^{2}$ losses (W) & 34 & 33 \\
\hline Transformer DC, $\left.R\right|^{2}$ losses (W) & \multicolumn{2}{|c|}{$\mathbf{6 6 . 4 6}$} \\
\hline
\end{tabular}

\subsubsection{Short circuited transformer AC resistance}

In order to evaluate the effect of frequency on the losses, the $A C$ resistances are measured for the frequency range of $100 \mathrm{~Hz}$ to $10 \mathrm{kHz}$ using an Agilent E4980A RLC meter. As it can be seen in Figure 6-8, the change of AC resistance is not significant below $1 \mathrm{kHz}$. It is shown in Table 6-11 that the losses calculated from theses measured $A C$ resistance are in agreement with the values resulted from FEM simulations.

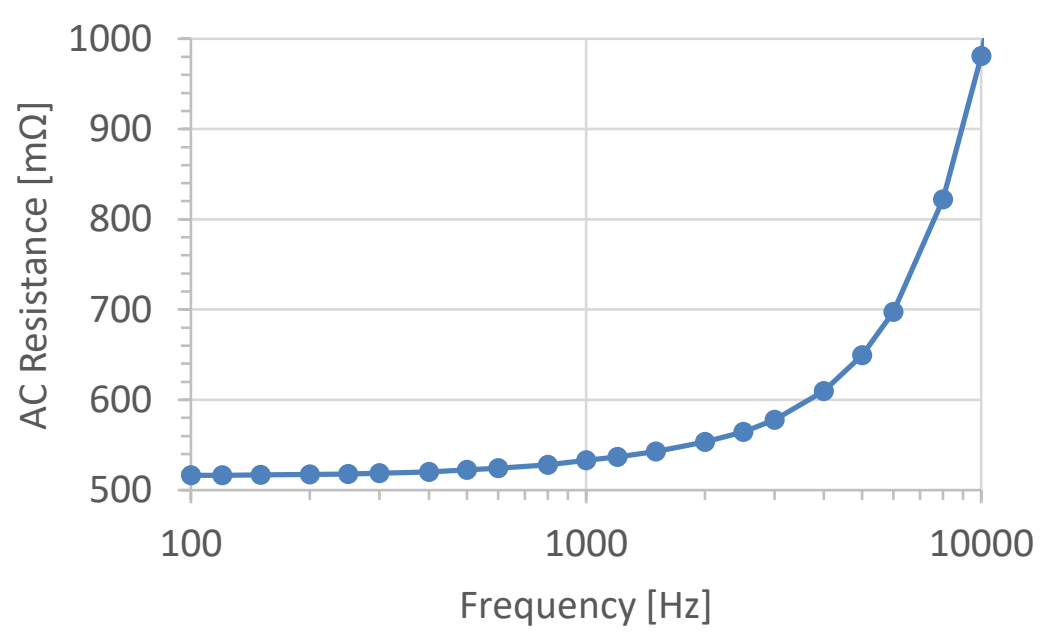

Figure 6-8. Series inductance and AC resistance measured from the secondary side while the primary side is shortcircuited 


\subsubsection{Load losses}

The winding loss measurement circuit is demonstrated in Figure 6-9.

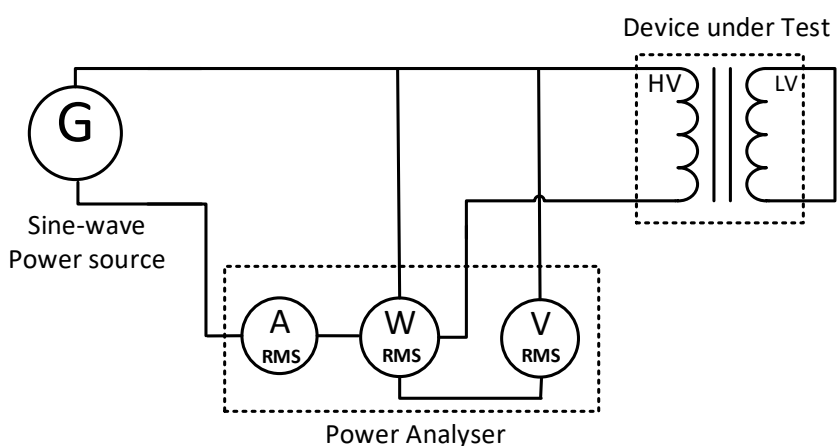

Figure 6-9. Windings loss measurement circuit diagram

The voltage, current and the power parameters were registered using a precision power analyser. The supply source used was a Chroma 61605 AC, 0-1000 Hz power supply (see Figure 6-10). To measure the total losses in the primary and secondary windings, the rated current of the transformer shall be applied to the windings. At the secondary side, when the primary side is short-circuited (see Figure 6-10), the voltage is raised until the maximum current, near to the rated current, passes simultaneously in both windings. The losses are measured as fast as possible to capture the losses at room temperature.

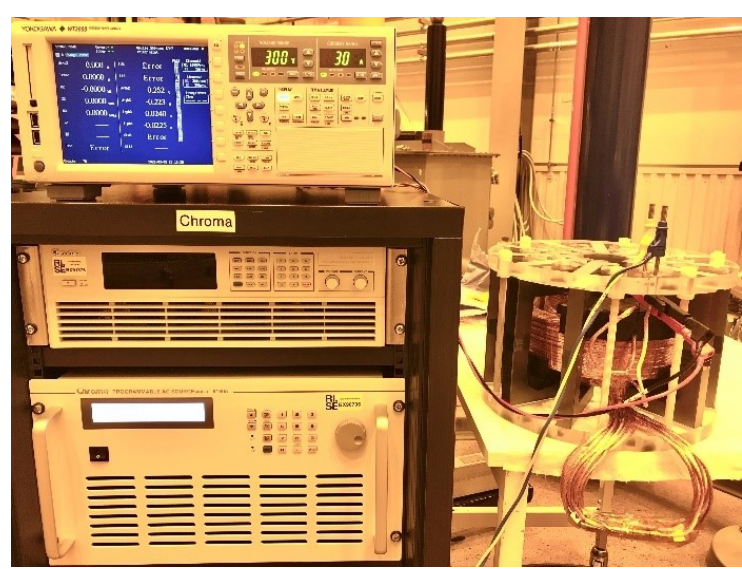

Figure 6-10. Windings loss measurement setup on prototype MFT

The load loss is measured at room temperature using the short circuit method and the DC resistance values at room temperature $\left(20^{\circ} \mathrm{C}\right)$ is used to estimate the load losses at the operation temperature (reference $80^{\circ} \mathrm{C}$ ). The load losses under full loading were calculated at rated current and reference temperature from the reduced current and room temperature measurements according to Table 6-9.

Table 6-9. Load losses at rated current and reference temperature $\left(80^{\circ} \mathrm{C}\right)$ at $1 \mathrm{kHz}$ from room temperature measurements

\begin{tabular}{|l|c|}
\hline Total $R l^{2}$ losses at $20^{\circ} \mathrm{C}$ & 66.46 \\
\hline Calculated $R I^{2}$ losses at $80{ }^{\circ} \mathrm{C}$ & 81.98 \\
\hline Measured Load losses at $20^{\circ} \mathrm{C}$ & 67.52 \\
\hline Additional Losses (measured total losses minus calculated $R I^{2}$ losses at $\left.20^{\circ} \mathrm{C}\right)$ & 1.06 \\
\hline Calculated Load losses at $80^{\circ} \mathrm{C}$ (calculated additional losses plus calculated $R I^{2}$ losses at $80^{\circ} \mathrm{C}$ ) & $\mathbf{8 3 . 0 4}$ \\
\hline
\end{tabular}

The losses in the extra wires out of the winding are considered according to Table 6-10.

Table 6-10. The losses in extra wires

\begin{tabular}{|c|c|c|c|c|}
\hline Extra wire & $\mathrm{mm}$ & $\mathrm{m} \Omega$ & $\mathrm{A}$ & $\mathrm{W}$ \\
\hline LV & 400 & 0.159 & 118.5 & 2.23 \\
\hline HV & 1800 & 5.724 & 11.11 & 0.71 \\
\hline Total & & & & 2.94 \\
\hline
\end{tabular}


The numerically calculated values are corrected to include the losses in the extra wires out of the winding and the $4 \%$ extra length of the Litz wire strands. It means that the calculated value should be added with 2.94 and the result should be multiplied by 1.04 . Table $6-11$ demonstrates the rise of AC resistance measured from the secondary side while the primary side was short-circuited. The resulting increase in the calculated losses in comparison with the losses directly measured, and also calculated by the numerical method, are also presented.

Table 6-11. The rise in AC resistance and calculated winding losses at $20^{\circ} \mathrm{C}$ compared with the measured value

\begin{tabular}{|c|c|c|c|c|}
\hline $\begin{array}{c}\text { Frequency } \\
(\mathrm{Hz})\end{array}$ & $\begin{array}{c}\text { Measured } \\
\text { AC resistance } \\
\text { from HV side }(\mathrm{m} \Omega)\end{array}$ & $\begin{array}{c}\text { Losses calculated } \\
\text { from AC } \\
\text { resistance }(\mathrm{W})\end{array}$ & $\begin{array}{c}\text { Losses Measured } \\
\text { by Power } \\
\text { Analyzer }(\mathrm{W})\end{array}$ & $\begin{array}{c}\text { Losses } \\
\text { Calculated } \\
\text { by FEM } \\
(\mathrm{W})\end{array}$ \\
\hline 1000 & 533 & 65.8 & 67.5 & 67.2 \\
\hline 2000 & 553 & 68.3 & - & 68.9 \\
\hline 3000 & 578 & 71.3 & - & 71.6 \\
\hline 4000 & 610 & 75.3 & - & 75.3 \\
\hline 5000 & 649 & 80.1 & - & $\mathbf{8 0 . 1}$ \\
\hline
\end{tabular}

Additionally, as demonstrated in Table 6-12, the total impedance of the short-circuited transformer calculated by the measured $R$ and $L$ values achieved from the RLC meter is comparable with the calculated impedance from the voltage and current values registered by the Power analyser.

Table 6-12. Comparison of the impedance measured by the RLC meter and during the winding loss measurements

\begin{tabular}{|l|c|}
\hline \multicolumn{1}{|c|}{$1 \mathrm{kHz}$} & Decagon \\
\hline$L(\mathrm{nH})$ & 3023335 \\
\hline$R(\mathrm{~m} \Omega)$ & 532.86 \\
\hline$Z(\Omega)$, RLC meter & $\mathbf{1 8 . 9 9}$ \\
\hline$U(\mathrm{~V})$ & 215.21 \\
\hline$I(\mathrm{~A})$ & 11.34 \\
\hline$Z(\Omega)$ at rated current & $\mathbf{1 8 . 9 8}$ \\
\hline
\end{tabular}

As a direct supply of the rated current under short circuit condition at $5 \mathrm{kHz}$ was not possible, putting all the above-mentioned data together, the load losses of the Decagon transformer at $80^{\circ} \mathrm{C}, 5 \mathrm{kHz}$ and the rated current will be $95.6 \mathrm{~W}$.

\subsection{Tests on insulation prototype}

To verify the insulation design, the insulation prototype is subjected to a high voltage DC stress test. The duration of voltage application was determined by the results of the simulation showing that a dynamic voltage distribution can continue up to 4000 seconds (see Figure 4-6).

The test circuit is shown in Figure 6-11. The applied voltage was measured by a precise $150 \mathrm{kV}$ DC voltage divider and was registered using a data acquisition system (Figure 6-12). The transformer passed successfully the test and no breakdown occurred during HVDC application. 


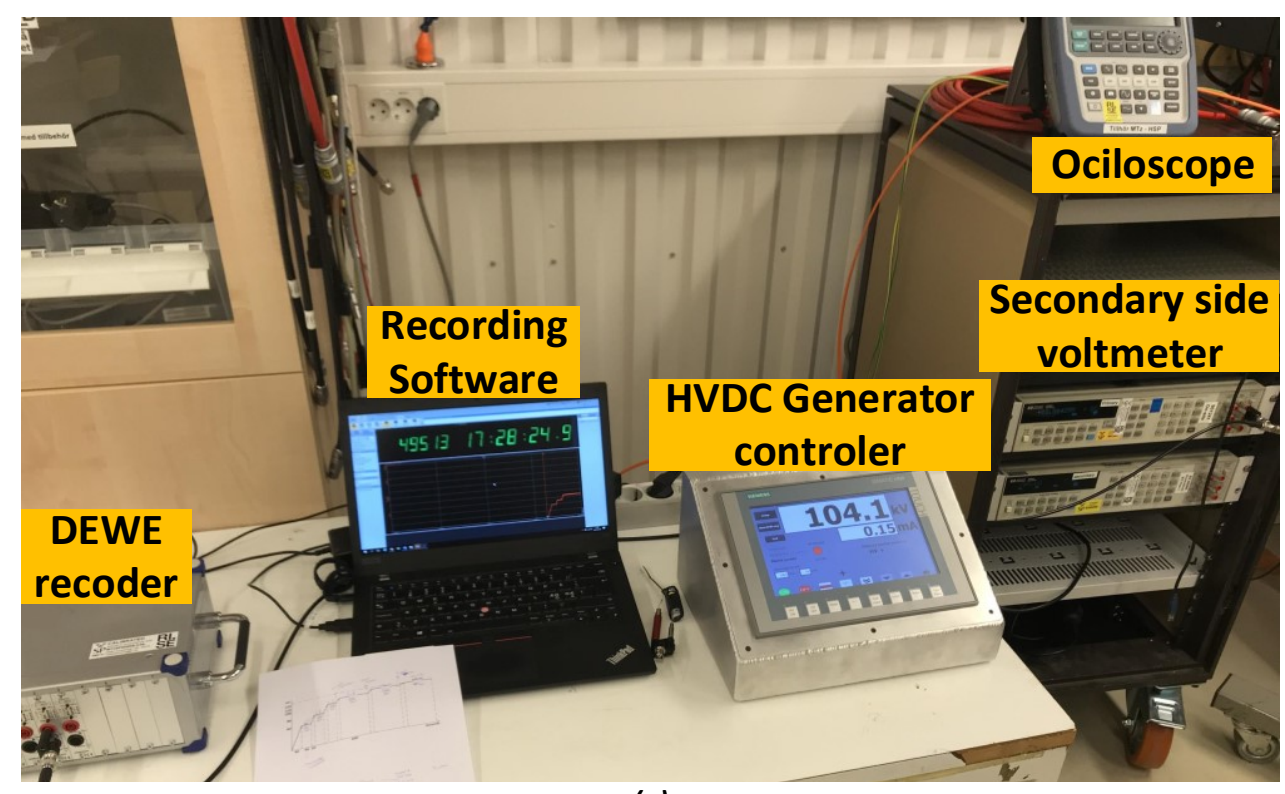

(a)

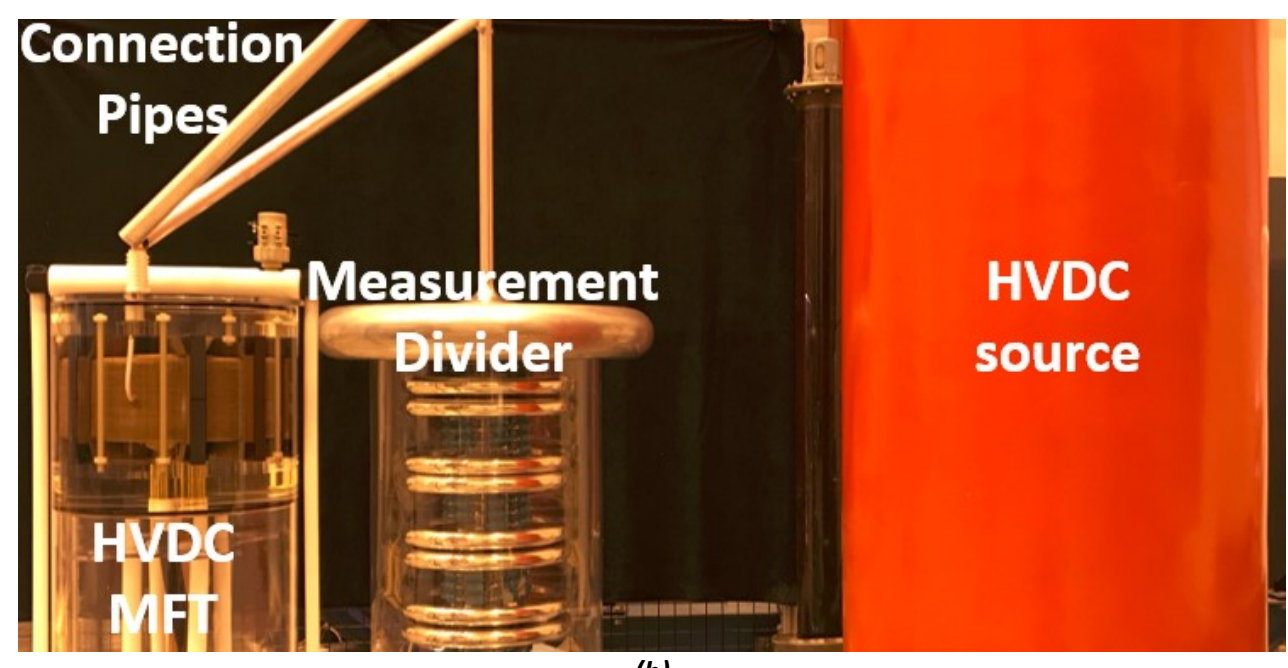

(b)

Figure 6-11. Insulation prototype HVDC testing setup; HVDC voltage control \& measuring setup (a), High voltage test setup (b)

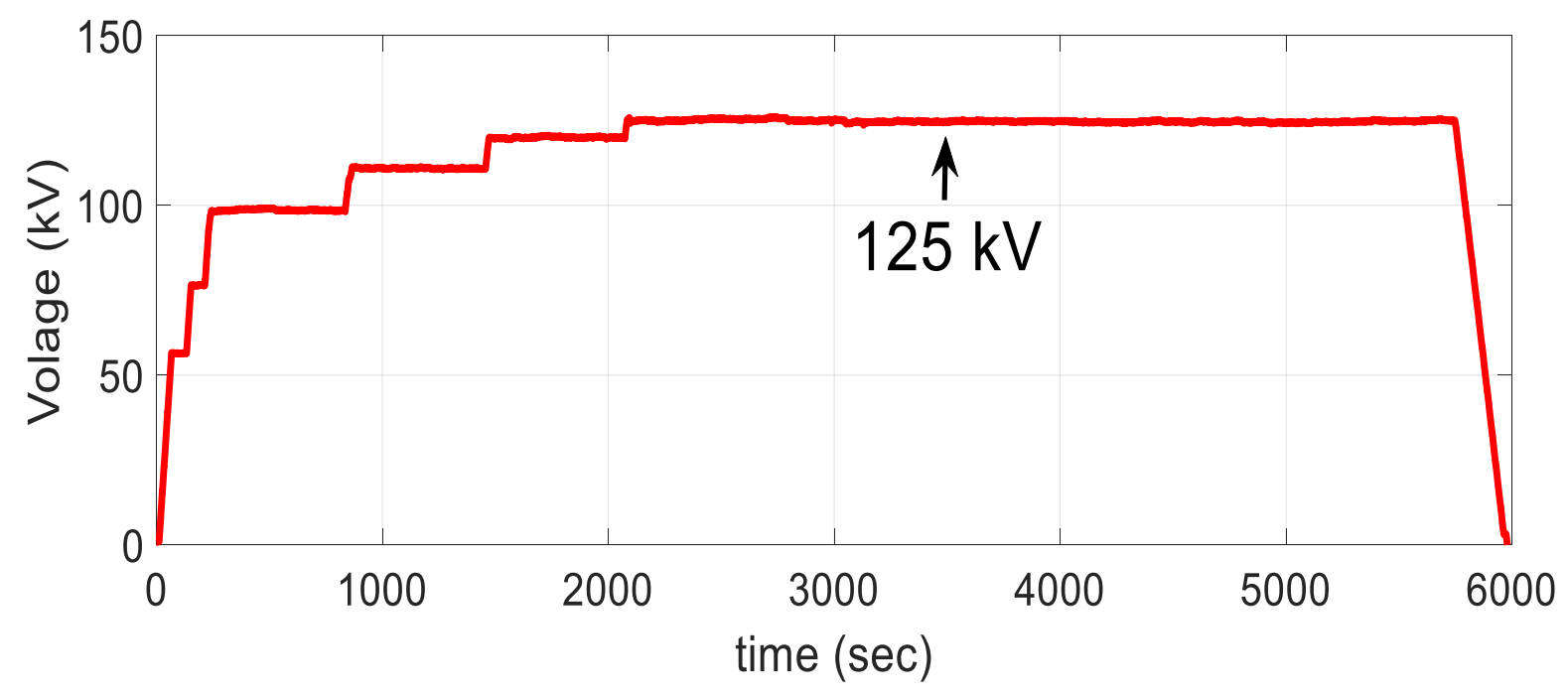

Figure 6-12. Applied voltage-time to HVDC MFT prototype 


\section{Design proposal for a full-scale unit}

A Master thesis titled "Feasibility study of sea-based wind park" [110] was conducted. For different offshore wind parks of types $A C / A C, A C / D C$ and $D C / D C$, the life cycle cost and the energy efficiency were determined and compared. In addition, the energy losses and costs of the transmissions systems were evaluated.

Three cases were selected and Life Cycle Cost (LCC) analysis was performed. Case 1 and case 2 were $1000 \mathrm{MW}$ and $500 \mathrm{MW}$ offshore wind parks respectively, located at four different distances form suitable grid connection points, namely 60, 80, 100 and $120 \mathrm{~km}$. Case 3 was a wind park similar to the $110 \mathrm{MW}$ Lillgrund park having 48 wind turbines of, $3 \mathrm{MW}$ located at two different distances out in the sea, namely 9 and $60 \mathrm{~km}$.

The findings from [110] show that for the wind turbines themselves, a weight saving of 3 tons was found for the nacelle transformer itself, which leads to a further reduced weight for the structure and foundations, totally about $4 \%$ for the plant as a whole. For the investigated oil-insulated transformer topology, it appeared that it seems realistic to reach a DC offset voltage of $\pm 150 \mathrm{kV}$. In addition, the DC series also offers the highest electrical efficiency related to the $A C / A C$ and $A C / D C$ systems. Due to the high energy losses in the $A C$ transmission system, i.e. in the cables, the $A C / A C$ system is more expensive than the AC/DC case, when the farm was located at a longer distance to land than $80 \mathrm{~km}$ to $100 \mathrm{~km}$. Subsequently, for large power capacities at long distances, the HVDC technology (AC/DC or DC/DC) is preferred.

In case of a park having the size of Lillgrund and is placed $9 \mathrm{~km}$ out in the sea an AC/AC system is more beneficent than the DC Series system. When the distance to the land increases (to $60 \mathrm{~km}$ for example), the DC Series will be more cost effective where the offshore platform needed is omitted. In this case $85 \mathrm{M} €$ will be saved. The efficiency of the DC design is also higher, and the losses will be 2 MW less than the similar AC/AC design.

In reference [110], among other studies, todays classical system, shown in Figure 7-1, is compared with a full scale series DC system (see Figure 7-2).

The interested reader can find more information in [111-115]. 


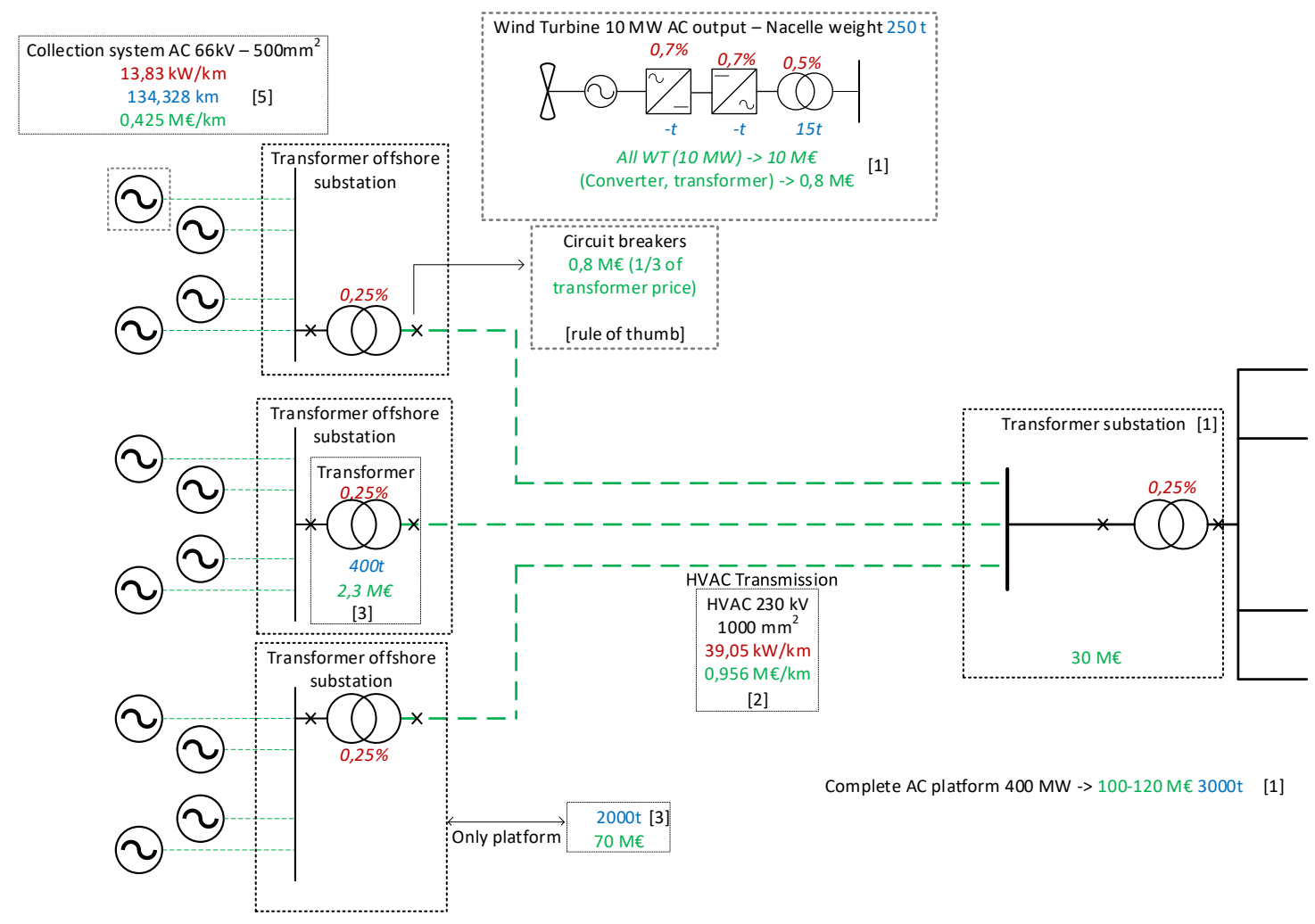

Figure 7-1. A detailed cost/Loss data of an AC/AC 1GW park having 100 WTs of 10 MW capacity

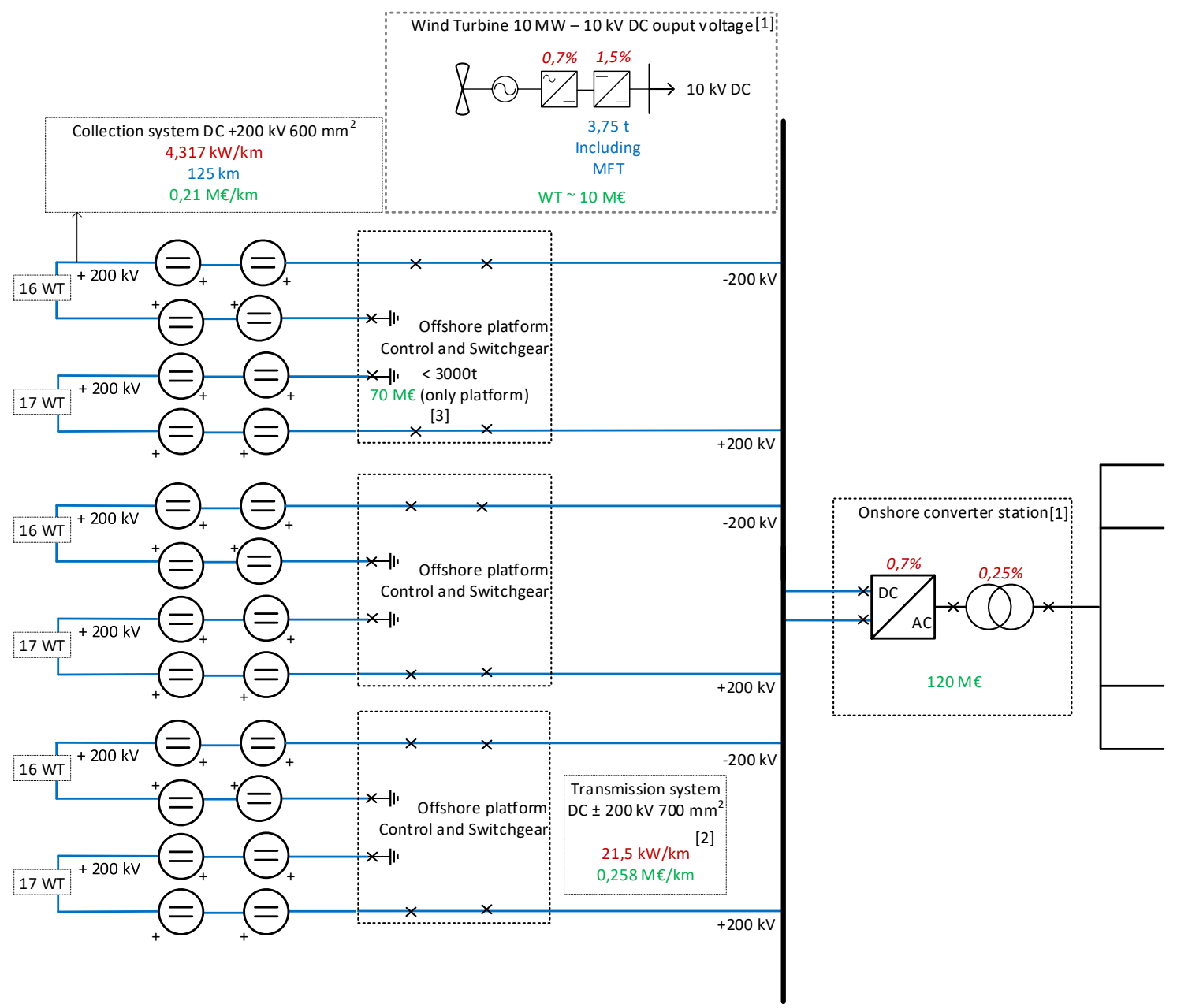

Figure 7-2. A detailed cost/Loss data of an DC Series 1 GW park having 100 WTs of 10 MW capacity 
In the system study the prerequisites of a $1 \mathrm{GW}$ farm having 100, $10 \mathrm{MW}$ turbines with up to $200 \mathrm{kV}$ HVDC insulation ability from the HV winding to ground is assumed. To realise this a series connection of $17,10 \mathrm{MW}, 1.8 / 18 \mathrm{kV}$ DC/DC converters with $250 \mathrm{kV} \mathrm{DC}$ to ground insulation will deliver up to 306 $\mathrm{kV}$ in series which has a good margin in case of uneven power production from the various turbines [Due to the current series connection, the turbines add voltage in proportion to their power production] or having a turbine out of circuit. $250 \mathrm{kV} \mathrm{HVDC}$ insulation ability from the HV winding to ground will be suitable for a system operating at maximum 200 KV DC to ground. Below, thus, follows the design proposal for such a full-scale unit is presented.

With this as background a full-scale prototype transformer for such a DC/DC converter is herewith proposed. $10 \mathrm{MW}, 1.8 / 18 \mathrm{kV} 5 \mathrm{kHz}$ AC, $250 \mathrm{kV}$ DC prototype, with a ferrite core:

- Rated primary current, $I_{T 1}$ (r.m.s.) $=10 \mathrm{MW} / 1.8 \mathrm{kV}=5555 \mathrm{~A}$

- Number of turns, $N_{1}$ and $N_{2}=12$ and 120

$\circ$ (For the winding type demonstrated in Figure 4-2, the highest voltage difference between two adjacent discs of 6 turns/disc will be $18 \mathrm{kV} / 120 \times 6 \times 2=1800 \mathrm{~V}$ and this means a vertical distance should be considered between two discs which are selected as $3 \mathrm{~mm}$ in this design.)

- Magnetic core cross section, calculated from (2-30) as $23437 \mathrm{~mm}^{2}$ for the case of using Ferrite material where

- $V_{r m s 1}$ is the rated voltage of the primary winding $=1800 \mathrm{~V}$

- $K_{c}$ is the core filling factor $\approx 1$ for the Ferrite cores

- $\quad N_{1}$ is the number of turns of the primary winding $=12$

$\circ B_{m}$ is the maximum working flux density of the core $\approx 0.32 \mathrm{~T}$ for the Ferrite

$\circ \quad f$ is the switching frequency $=5[\mathrm{kHz}]$

- By switching from $125 \mathrm{kV}$ for the prototype to $250 \mathrm{kV}$ the electrical field in the core window will be much higher. The isolation distances shall be increased, compared with the prototype case. As explained in section 2.2.4, this means a higher number of barriers and oil gaps. The oil gap distances, the OIP barriers thicknesses and the number of barriers were increased. Using the developed method earlier demonstrated in section 4.2, after a series of simulations, the oil gap distances of $6 \mathrm{~mm}$, the OIP barrier thicknesses of $6 \mathrm{~mm}$ and a number of barriers of 4 are selected.

- As the voltage levels are 4 times higher than the prototype MFT case, the core area is selected similar to the prototype with 2 times increase in the dimensions. The core will have $40 \times 56=2240 \mathrm{~mm}^{2}$ as physical core area and similar to the prototype case, 10 core stacks including $20 \mathrm{U}$ cores is found to be suitable for the full-scale design.

- A Litz wire $9000 \times 0.2 \mathrm{~mm}$ having the physical dimension of $26.6 \times 17.5 \mathrm{~mm}^{2}$ is assumed to be used. This is a $7 \times 7$ wires combination of the previously used $3.8 \times 2.5$ Litz wires.

- For the primary layer type winding, considering $5555 \mathrm{~A}$ as the rated r.m.s. current, the space that the primary winding occupy in the core window will have a height of $851 \mathrm{~mm}$ (consisting of eight $26.6 \mathrm{~mm}$ parallel wires on top of the each other) and the width of $130 \mathrm{~mm}$ (consisting of four $17.5 \mathrm{~mm}$ layers having a $20 \mathrm{~mm}$ oil gap between the layers for the cooling purpose). Considering 3 turns of the 8 parallel $26.6 \times 17.5 \mathrm{~mm}^{2}$ Litz wires on the top of each other, the ideal magnetic height of the winding will be $638 \mathrm{~mm}$. For eight parallel $9000 \times 0.2$ $\mathrm{mm}$ Litz wire the actual current density will be $2.5 \mathrm{~A} / \mathrm{mm}^{2}$.

- For the secondary disc type winding, considering $555.55 \mathrm{~A}$ as rated r.m.s. current, the space that the secondary winding occupy in the core window need a height of $589 \mathrm{~mm}$ (consisting 
of twenty $26.6 \mathrm{~mm}$ discs having $3 \mathrm{~mm}$ oil gap between the discs for the electrical isolation and for cooling purpose) and a width of $115 \mathrm{~mm}$ (consisting of six $17.5 \mathrm{~mm}$ wires on the side of the each other having $2 \mathrm{~mm}$ gaps for the cooling purpose between turns). The actual current density will be $1.97 \mathrm{~A} / \mathrm{mm}^{2}$. The dimensions of the real scale $10 \mathrm{MVA} \mathrm{HVDC} \mathrm{MFT}$ are presented in Figure 7-3.

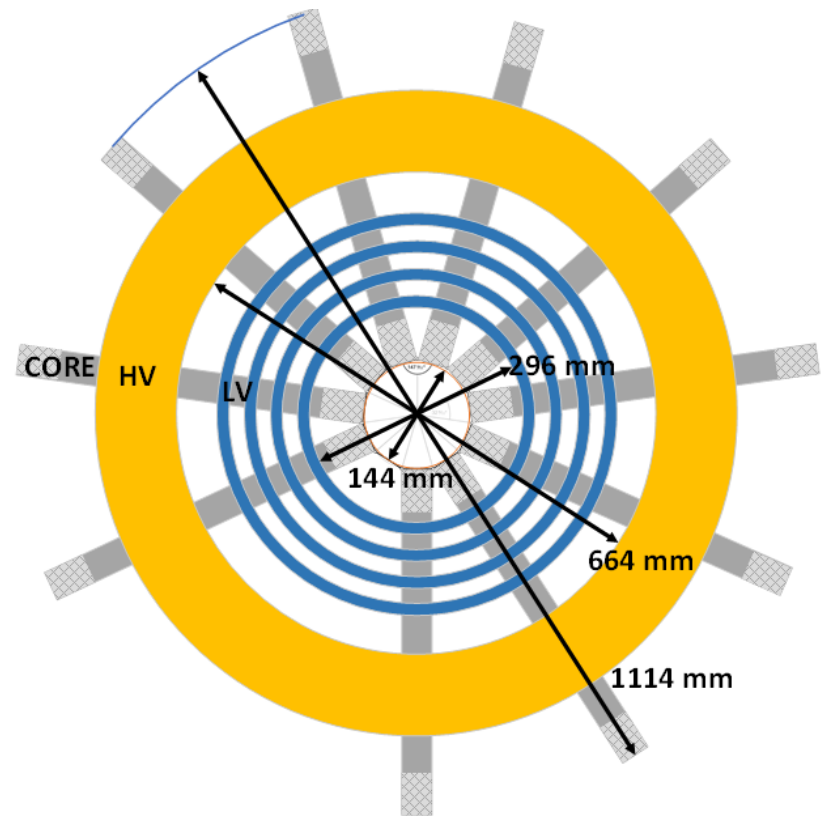

(a)

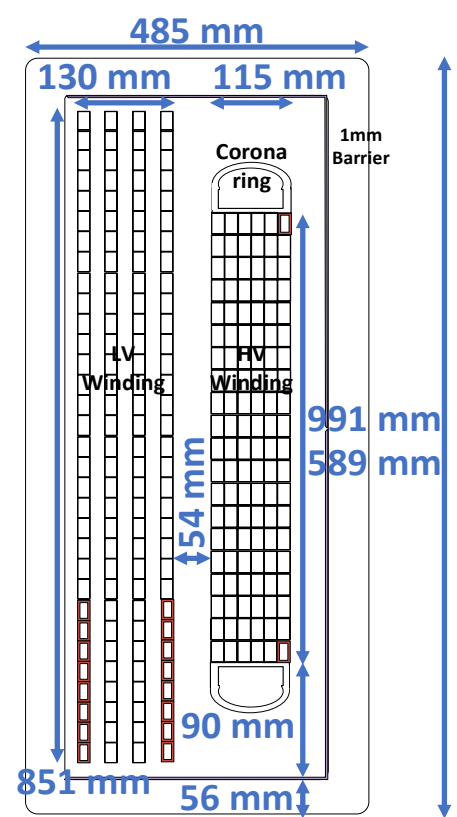

(b)

Figure 7-3. Dimensions of the real scale Ferrite $10 \mathrm{MW}, 1.8 \mathrm{kV} / 4.5 \mathrm{kV}, 0.32 \mathrm{~T}, 5555$ A/555 A, 12/120 Turns, $5 \mathrm{kHz}$ MFT

The weight of the transformer can be calculated as the sum of the weights of windings, oil and the cores. The weight of the windings will be $1320 \mathrm{~kg}$ as the total volume of the LV and HV windings will be 0.080 and $0.166 \mathrm{~m}^{3}$ respectively, the fill factor is 0.6 and the density of the copper wire is 8.96 $\mathrm{g} / \mathrm{cm}^{3}$. The volume of the core will be $0.061 \mathrm{~m}^{3}$ and the weight of core will be $297.6 \mathrm{~kg}$ as the density of the core is $4870 \mathrm{~kg} / \mathrm{m}^{3}$. The volume of the cylindrical tank will be $0.983 \mathrm{~mm}^{3}$ with the base diameter of $1.1 \mathrm{~m}$ and height of $0.991 \mathrm{~m}$ and therefore, the volume of the oil will be $0.63 \mathrm{~m}^{3}$ and the weight of the oil will be nearly $570.8 \mathrm{~kg}$. Thus, the total weight of the transformer will be nearly 2.2 ton.

For a quick comparison, a sample three phase $10 \mathrm{MVA} 50 \mathrm{~Hz} 6.3 / 33$ transformer can have a dimension of $\mathrm{L} 2.9 \times \mathrm{W} 1.4 \times \mathrm{H} 2.2 \mathrm{~m}^{3}$ and a weight of 26 tons. More examples are presented in Table 7-1.

Table 7-1. Examples of $50 \mathrm{~Hz}$ 3-phase transformers in a similar range to the designed real scale $10 \mathrm{MVA}$ transformer

\begin{tabular}{|l|c|c|c|}
\hline Voltage $(\mathrm{kV})$ & $6.6 / 20$ & $20 / 20$ & $6.3 / 33$ \\
\hline Weight $($ ton $)$ & 20.6 & 22.3 & 21.2 \\
\hline Length $(\mathrm{m})$ & 3.5 & 3.3 & 3.4 \\
\hline Width $(\mathrm{m})$ & 2.4 & 2.2 & 2.8 \\
\hline Height $(\mathrm{m})$ & 2.8 & 3.0 & 2.9 \\
\hline Tank volume $\left(\mathrm{m}^{3}\right)$ & 23.5 & 21.8 & 27.6 \\
\hline
\end{tabular}

To have a safe distance from the ground in case of application $250 \mathrm{kV} \mathrm{DC}$ step, the highest voltage for the equipment $\left(U_{m}\right.$, r.m.s. value) of $170 \mathrm{kV}(\approx 250 / \mathrm{V} 2)$ can be selected and according to IEC EN 600711:2006 [116] the insulation distance of the high voltage outputs from ground shall be selected as 1.5 meters. This is true in the case of standard reference atmospheric conditions [116], where the temperature is $20^{\circ} \mathrm{C}$, the pressure is $1013 \mathrm{mbar}$ and the absolute humidity is $11 \mathrm{~g} / \mathrm{m}^{3}$. In addition, 
the ambient air shall be not polluted by dust, vapours or salt. These means that in case a construction like the prototype transformer is selected at reference atmospheric condition the minimum dimensions of a container carrying the transformer will not be smaller than $3 \times 3 \times 3 \mathrm{~m}^{3}$ (see Figure 7-4).

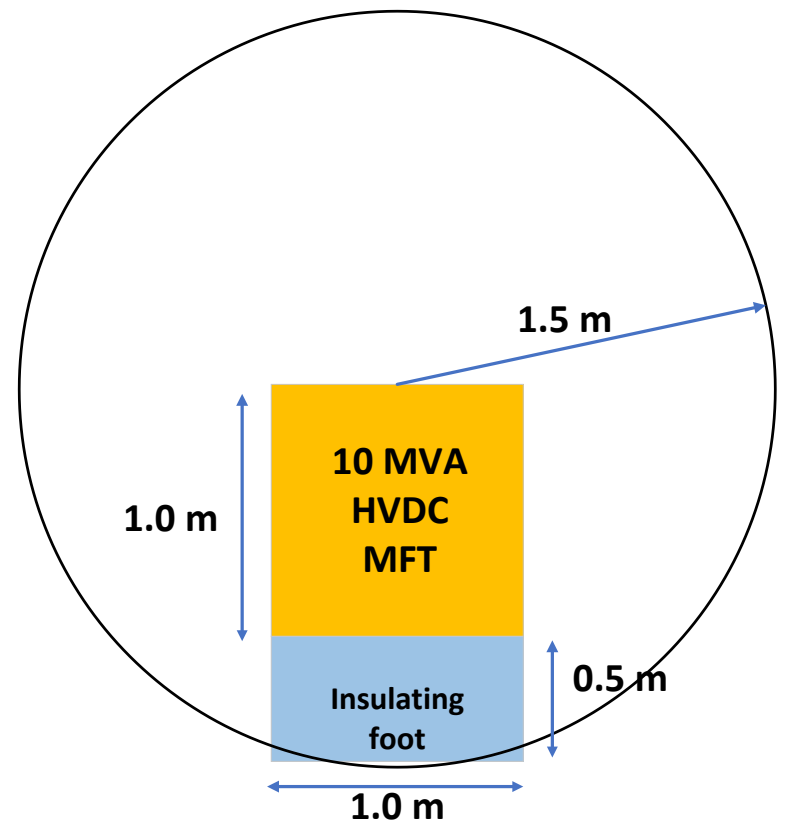

Figure 7-4. The safe clearance in air for a $250 \mathrm{kV}$ DC MFT

The converter can also be placed inside a monopile tubular steel foot which is the most common foundation for offshore wind turbines and normally have the diameter of 4 to 6 metres. The other solution is to use a direct cable connector to the metal transformer tank. An example is a set of HVCONNEX separable connectors, size 6, which are designed to work with an AC voltage up to $170 \mathrm{kV}$ and a current up to $2500 \mathrm{~A}$. Special components with corresponding material properties suitable for offshore applications can be ordered. In this case there will be no need for external clearances in air as a shielded cable is connected directly to a grounded transformer tank (see Figure 7-5).

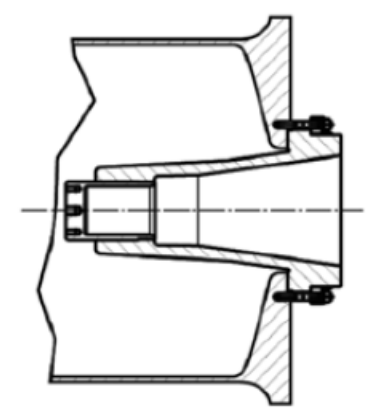

(a)

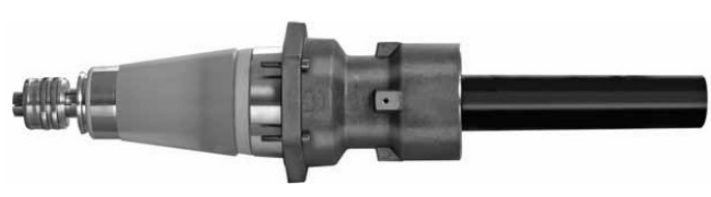

(b)

Figure 7-5. A HV-CONNEX separable connectors schematic [117]; Female coupling on metal transformer tank (a), Cable termination directly pluggable to the tank (b)

\section{Insulation design of the Real scale transformer}

Since both conductivity measurement data both for the mineral oil and ester are available in this stage, the Comsol simulations are performed to consider insulation strength if the same mechanical design is filled with the mineral or with the ester oil. Figure 7-6 shows the electric field level in the different areas of the transformers for different times at $50{ }^{\circ} \mathrm{C}$. Referring to the simulation results presented in section 3.3.3 the ester transformer has reached to the final steady state situation just after 10 seconds. 


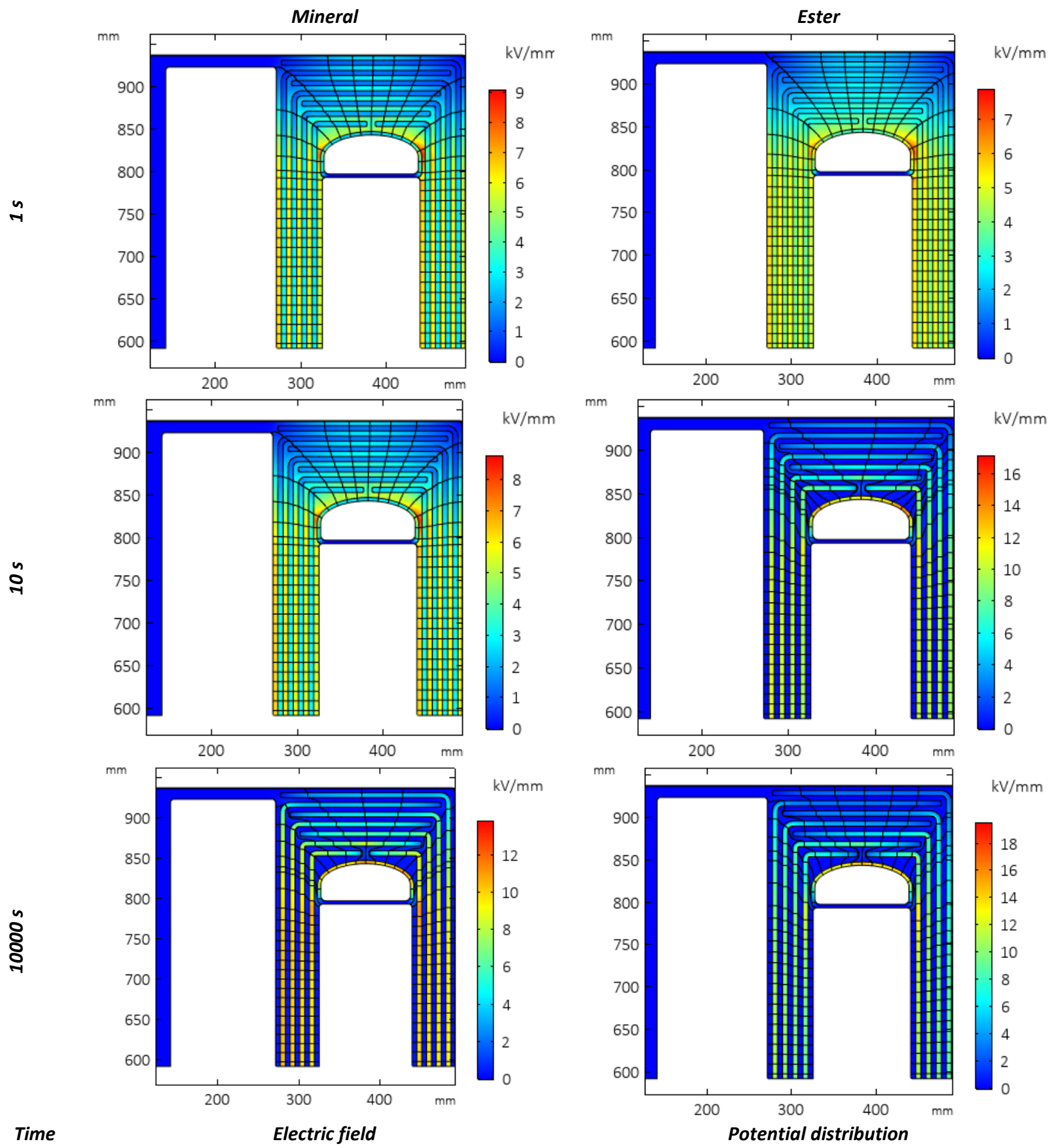

Figure 7-6. Electric filled in the mineral and ester filled 10 MVA transformers after 1,10 and 10000 seconds at $50{ }^{\circ} \mathrm{C}$

The summary of the simulations performed to detect the weak areas in the oil gaps and OIP barriers is presented in Table 7-2.

Table 7-2. A summary of the insulation design simulation for the electric field in the oil gaps and OIP

\begin{tabular}{|l|c|c|c|}
\hline & Temperature $\left({ }^{\circ} \mathrm{C}\right)$ & Maximum E-field in OIP $(\mathrm{kV} / \mathrm{mm})$ & Minimum SF in oil gaps \\
\hline \multirow{3}{*}{ Mineral oil and OIP } & 30 & 16.3 & 1.88 \\
\cline { 2 - 4 } & 50 & 13.7 & 1.88 \\
\cline { 2 - 4 } & 90 & 7.4 & 1.88 \\
\hline \multirow{3}{*}{ Ester oil and OIP } & 30 & 17.4 & 2.14 \\
\cline { 2 - 4 } & 50 & 18.2 & 2.15 \\
\cline { 2 - 4 } & 90 & 17.8 & 2.19 \\
\hline
\end{tabular}

The table shows that in case of the maximum Electric field (E-field) in the OIP barriers, the transformer filled with the mineral oil is safer; however, the variation of the E-field with temperature 
is higher and this means that the stress in the oil gaps will be changing with the loading and unloading of the transformer which can be interpreted as an unfavourite feature. Regarding the Safety Factor (SF) the situation is not the same and the ester oil gaps are under lower stress compared with the mineral oil. It should be mentioned that, as the maximum oil gap strength curves were not available for the ester oil, based on the similar AC breakdown test levels reported in literature $[118,119]$, the same maximum oil gap strength curves are used both for the mineral and ester oils. Figure 7-7 shows the regions with the lowest SF or the highest E-field detected for the mineral and ester oil field transformers occurred.

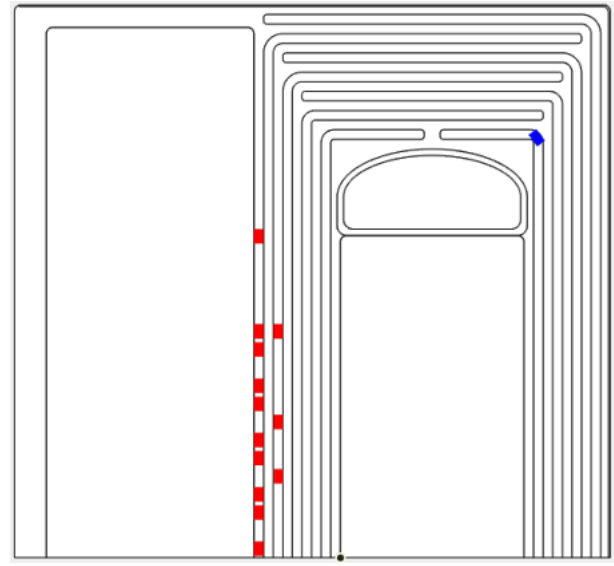

(a)

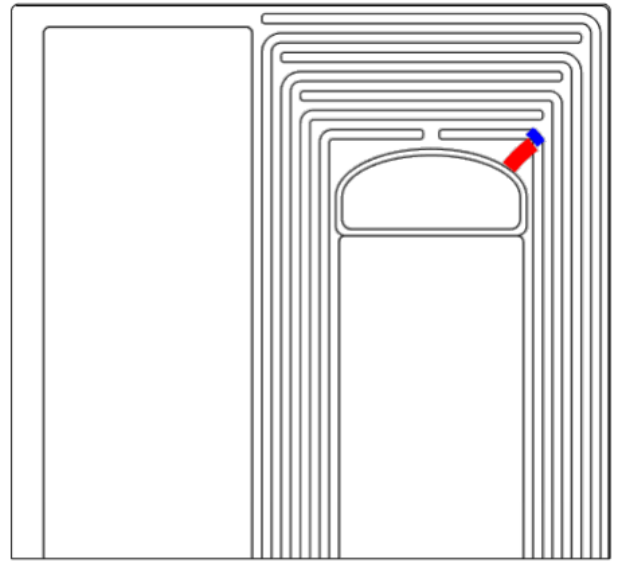

(b)

Figure 7-7. The critically stressed regions in the oil (red) and in the OIP barriers; The transformer filled with the mineral oil (a) and ester oil (b)

Figure 7-8 shows the regions with the lowest SF detected for the creepage fields in the mineral and ester oil transformers occurred. The summary of the simulations performed to detect the weak creepage areas in the oil-OIP interfaces is presented in Table 7-3.

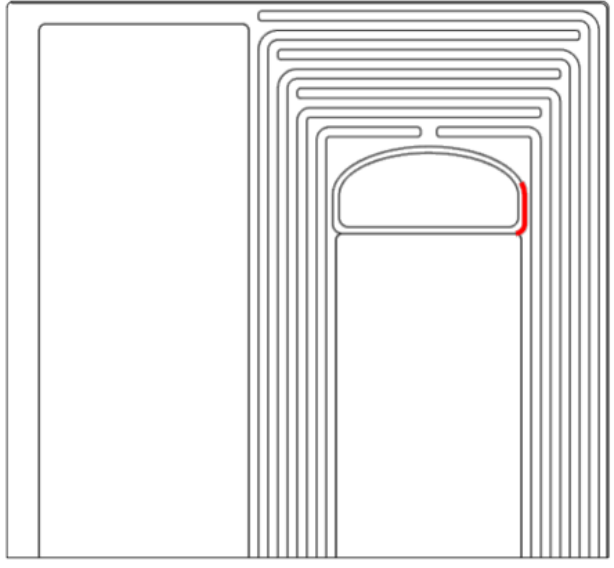

(a) The transformer filled with the mineral oil

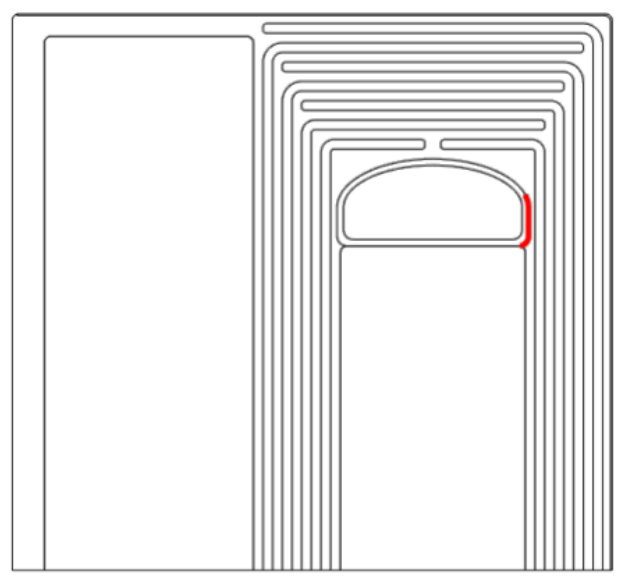

(b) The transformer filled with the ester oil

Figure 7-8. The critically stressed regions in the oil/OIP interfaces (red); The transformer filled with the mineral oil (a) and ester oil (b)

Table 7-3. A summary of the insulation design simulation for the creepage electric field in the oil-OIP interfaces

\begin{tabular}{|l|c|c|}
\hline & Temperature $\left({ }^{\circ} \mathrm{C}\right)$ & Minimum SF on the creepage paths \\
\hline \multirow{3}{*}{ Mineral oil and OIP } & 30 & 2.17 \\
\cline { 2 - 3 } & 50 & 2.15 \\
\cline { 2 - 3 } & 90 & 2.44 \\
\hline \multirow{3}{*}{ Ester oil and OIP } & 30 & 2.13 \\
\cline { 2 - 3 } & 50 & 2.18 \\
\cline { 2 - 3 } & 90 & 2.24 \\
\hline
\end{tabular}


At the same time the leakage inductance dictated from the converter side shall be considered. This makes a restriction for the dimensions of the windings and their distance from each other. In addition, the issue of cooling for the windings should be investigated. The windings have higher current densities in case of the real scale transformer. The thermal design is not covered by this thesis, but it is natural that before the placements of the insulation barriers and the oil gaps, is made, the flow of the cooling transformer oil inside the active part shall be investigated. 


\section{Conclusions and future work}

\subsection{Conclusions}

This research presents solutions to actualise the technology required for the direct connection of offshore wind farms to land, without the need for a very large and expensive $50 \mathrm{~Hz}$ transformer station. It introduces a method for the insulation design of a HVDC medium frequency oil-paper insulated power transformer, which is the key element inside DC/DC converters connected to the outputs of the wind turbines.

First, as a fundamental input to the insulation design, specialised measurements for HVDC conductivity of insulation materials have been performed and the results are compared with reliable sources. Then, the data was used as input for Multiphysics simulations, using Comsol software and the non-linear Maxwell Wagner theories. Finally, a prototype was built and tested to verify the results of simulations.

The key result of this study is the development of an insulation design methodology for a HVDC insulated Medium Frequency Transformer (MFT).

This cross disciplinary research introduces new solutions for power-electronic researchers, and a new application for high-voltage designers. The developed method in this work can be easily implemented for more complicated designs of HVDC MFTs and facilitates the application of these step-up transformers for power electronic engineers where higher isolation levels are required.

The study shows that for dry-type transformers, it is difficult to accomplish with a relatively high voltage to ground insulation. It is shown in this work that, an oil type transformer is the solution. For an oil-type transformer, the long oil gaps shall be divided by several solid barriers. It is verified that, compared with normal AC transformers, for a DC design, the thickness of solid barriers shall be increased. It is explained how the position, form and the overlapping of the solid barriers shall be considered.

It is revealed that for a successful DC insulation design, the conductivity of the insulation materials should be determined. The measurements, made in this work, demonstrate that the conductivities of transformer oil and OIP exhibit strong nonlinear dependencies on temperature and the electric field that makes the performance of the insulation of the MFT, dependent on specific operation conditions.

A simple comparison of the achieved conductivity values of ester and mineral oil and OIP highlights that the conductivity ratio of the ester oil/OIP are not as much temperature and voltage stress dependent as conductivity ratio of the mineral oil/OIP is. This means a reduced dynamic stress level of the design in case of using ester oil and OIP. It is also shown that the ester oil/OIP conductivities are approximately 100-1000 times higher than mineral oil/OIP conductivities. This, results in a much shorter time for the ester transformer to reach the steady state condition.

In addition, presented results of the high frequency breakdown tests show that the characterization of the insulation of the delivered materials like Litz wires or insulation tapes is vital before a successful insulation design of a medium frequency transformer. 
Additionally, a parametric field and temperature dependent stress investigation, focusing on all the weak points of the transformer insulation are performed and it is strongly recommended for a reliable HVDC oil paper insulation system design process. The nonlinear dependency of the oil and OIP to the applied voltage and temperature, is considered. This is crucial for an accurate calculation of the dynamic electric fields in the insulation system of the transformer.

Moreover, in this work it is demonstrated how the leakage inductance constraint shall be realised during the insulation design. An analytical method has been established for the computing of the leakage inductance. The final expressions are simple and fast convergent. Using the proposed method, time-consuming 3D FEM simulations can be avoided in the design stage without compromising with the accuracy.

Furthermore, an effective numerical method is utilized for calculation of eddy current losses in Medium Frequency Transformer (MFT) windings made by Litz wires. The numerical method is based on the equivalent permeability and conductivity of stranded copper conductors. The proposed method is effective and quick.

To sum up, the concept of the medium frequency transformer with a high DC insulation to ground thought to be used in the DC/DC converters for the series high voltage DC integration of the offshore wind farms, has been proven viable, and no obstacle to further progress have been identified.

\subsection{Future work}

Due to the project's low TRL level there are a great number of further studies that needs to be made, below, some of them are listed.

- Thermal design:

Finish the thermal design and perform a heat run verification test

- High frequency stress:

Continue with high frequency insulation aspect, perform high frequency induced over voltage test on a prototype transformer

- Biodegradable oil:

Continue with the biodegradable oil, redesign the prototype considering the thermal and electrical insulation aspects test the new prototype

- Conductivity measurements:

Continue with the conductivity measurements especially with the ester oil and Nomex paper

- Core losses:

Continue with core specification tests including determination of the actual loss values inside the cut cores of the same type, the effect of temperature on the saturation flux and loss density of the cores and effect of wave shape and frequency on 'Loss density - Flux density' curves

- Simulations:

Perform a combined Multiphysics thermal and electrical insulation simulation and analysis Perform 3D insulation design calculation to consider the high voltage exit leads and the effect of sharp core corners

- System level:

Study the degree of over-dimensioning needed for a stable DC output voltage needed to keep redundancy 
- High voltage tests:

Investigate the DC breakdown strength of combined oil/paper dielectric structure by experiments

Do BD tests on the synthetic ester oil and extract oil strength curves like the Weidmann withstand curves

Perform AC \& DC stress combined breakdown tests on insulation materials

Continue with the high voltage high frequency withstand tests using a voltage source having a square wave shape

- Other solid insulation materials:

Investigate the suitability of using a solid material like Teflon, PE, POM or Plexiglas inside oil considering the wetting effect of oil on the surface of them

- HVDC oil/paper insulation system:

Build a Kerr effect setup and perform measurements on the different oil-barrier-electrode arrangements

- Short circuit condition:

Perform short circuit forces calculations and verification tests on the Litz wires windings

- Transformer manufacturing:

Plexiglass can be charged on its surface, and discharges on its surface can happen. This can also be the case if we use glass. A semiconducting layer will help to prevent surface charge and discharge. Investigate applying a semiconductor pain on the surface of the Plexiglass tank

Do a new prototype design with a metal tank and plug-in high voltage cable termination 


\section{Bibliography}

[1] IEA. "IEA Press Release, Offshore wind to become a \$1 trillion industry." https://www.iea.org/news/offshore-wind-to-become-a-1-trillion-industry (accessed 25-082020).

[2] ABB. "ABB Review, Special Report, 80 years of HVDC." https://library.e.abb.com/public/aff841e25d8986b5c1257d380045703f/140818\%20ABB\%20 SR\%2060\%20years\%20of\%20HVDC 72dpi.pdf (accessed 25-08-2020).

[3] M. A. Bahmani, "Design and Optimization Considerations of Medium-frequency Power Transformers in High-power DC-DC Applications," Chalmers University of Technology, 2016. [Online]. Available: https://books.google.se/books?id=XG8CkAEACAAJ

[4] M. Kharezy, "Constructional Design, Manufacturing and Evaluation of High Power Density Medium Frequency Transformer Prototypes ", Chalmers University of Technology, 2014. [Online]. Available: http://publications.lib.chalmers.se/records/fulltext/211818/211818.pdf

[5] L. Heinemann, "An actively cooled high power, high frequency transformer with high insulation capability," in APEC. Seventeenth Annual IEEE Applied Power Electronics Conference and Exposition (Cat. No.02CH37335), 2002 2002, vol. 1, pp. 352-357 vol.1, doi: 10.1109/APEC.2002.989270.

[6] M. Steiner and H. Reinold, "Medium frequency topology in railway applications," in 2007 European Conference on Power Electronics and Applications, 2-5 Sept. 2007 2007, pp. 1-10, doi: 10.1109/EPE.2007.4417570.

[7] Spezial-Transformatoren-Stockach. "MF Transformer for Traction." http://www.ststrafo.com/fileadmin/user upload/messeposter rail kor2.pdf (accessed 25-08-2020).

[8] M. A. Bahmani, T. Thiringer, and M. Kharezy, "Optimization and experimental validation of medium-frequency high power transformers in solid-state transformer applications," in 2016 IEEE Applied Power Electronics Conference and Exposition (APEC), 20-24 March 2016 2016, pp. 3043-3050, doi: 10.1109/APEC.2016.7468297.

[9] M. Jaritz, S. Blume, and J. Biela, "Design procedure of a $14.4 \mathrm{kV}, 100 \mathrm{kHz}$ transformer with a high isolation voltage (115 kV)," IEEE Transactions on Dielectrics and Electrical Insulation, vol. 24, no. 4, pp. 2094-2104, 2017, doi: 10.1109/TDEI.2017.006279.

[10] T. B. Gradinger, U. Drofenik, and S. Alvarez, "Novel insulation concept for an MV dry-cast medium-frequency transformer," in 2017 19th European Conference on Power Electronics and Applications (EPE'17 ECCE Europe), 11-14 Sept. 2017 2017, pp. P.1-P.10, doi: 10.23919/EPE17ECCEEurope.2017.8099006.

[11] P. Huang, C. Mao, and D. Wang, "Electric field simulations and analysis for high voltage high power medium frequency transformer," Energies, vol. 10, no. 3, p. 371, 2017.

[12] G. Ortiz, "High-power DC-DC converter technologies for smart grid and traction applications," ETH Zurich, 2014.

[13] D. D. M. Mogorovic, "High Power MFT Design Optimization," École Polytechnique Fédérale de Lausanne, 2017.

[14] U. Gafvert, A. Jaksts, C. Tornkvist, and L. Walfridsson, "Electrical field distribution in transformer oil," IEEE Transactions on Electrical Insulation, vol. 27, no. 3, pp. 647-660, 1992, doi: 10.1109/14.142730.

[15] U. Gafvert, O. Hjortstam, Y. Serdyuk, C. Tornkvist, and L. Walfridsson, "Modeling and Measurements of Electric Fields in Composite Oil/Cellulose Insulation," in 2006 IEEE Conference on Electrical Insulation and Dielectric Phenomena, 15-18 Oct. 2006 2006, pp. 154157, doi: 10.1109/CEIDP.2006.312084.

[16] L. Yang, S. M. Gubanski, Y. V. Serdyuk, and J. Schiessling, "Dielectric properties of transformer oils for HVDC applications," IEEE Transactions on Dielectrics and Electrical Insulation, vol. 19, no. 6, pp. 1926-1933, 2012, doi: 10.1109/TDEI.2012.6396949. 
[17] K. Kato, H. Okubo, F. Endo, A. Yamagishi, and K. Miyagi, "Investigation of charge behavior in low viscosity silicone liquid by kerr electro-optic field measurement," IEEE Transactions on Dielectrics and Electrical Insulation, vol. 17, no. 4, pp. 1214-1220, 2010, doi: 10.1109/TDEI.2010.5539692.

[18] M. Wakamatsu, K. Kato, N. Inoue, H. Koide, and H. Okubo, "DC field measurement in Oil/Pressboard composite insulation system by electro-optic Kerr effect," IEEE Transactions on Dielectrics and Electrical Insulation, vol. 10, no. 6, pp. 942-947, 2003, doi: 10.1109/TDEI.2003.1255770.

[19] H. Okubo, "HVDC electrical insulation performance in oil/pressboard composite insulation system based on Kerr electro-optic field measurement and electric field analysis," IEEE Transactions on Dielectrics and Electrical Insulation, vol. 25, no. 5, pp. 1785-1797, 2018, doi: 10.1109/TDEI.2018.007187.

[20] Y. Ebisawa, S. Yamada, S. Mori, and T. Teranishi, "DC creepage breakdown characteristics of oil-immersed insulation," IEEE Transactions on Dielectrics and Electrical Insulation, vol. 16, no. 6, pp. 1686-1692, 2009, doi: 10.1109/TDEI.2009.5361590.

[21] R. Nakane, H. Okubo, and K. Kato, "HVDC electrical insulation performance based on charge activity in oil-pressboard composite insulation structures," IEEE Transactions on Dielectrics and Electrical Insulation, vol. 26, no. 2, pp. 576-583, 2019, doi: 10.1109/TDEI.2019.007682.

[22] M. Yea, K. J. Han, J. Park, S. Lee, and J. Choi, "Design optimization for the insulation of HVDC converter transformers under composite electric stresses," IEEE Transactions on Dielectrics and Electrical Insulation, vol. 25, no. 1, pp. 253-262, 2018, doi: 10.1109/TDEI.2018.006629.

[23] T. Gabler, K. Backhaus, S. Großmann, and R. Fritsche, "Dielectric modeling of oil-paper insulation systems at high DC voltage stress using a charge-carrier-based approach," IEEE Transactions on Dielectrics and Electrical Insulation, vol. 26, no. 5, pp. 1549-1557, 2019, doi: 10.1109/TDEI.2019.008175.

[24] H. P. Moser and V. Dahinden, "Transformerboard II, Properties and application of transformerboard of different fibers". Weidmann AG, 1987.

[25] M. J. P. Jeroense and F. H. Kreuger, "Electrical conduction in HVDC mass-impregnated paper cable," IEEE Transactions on Dielectrics and Electrical Insulation, vol. 2, no. 5, pp. 718-723, 1995, doi: 10.1109/94.469968.

[26] Waslström, Cigré, Electra, ELT_046_1, working group A2.12, "Voltage tests on transformers and smoothing reactors for HVDC transmission". 1976, pp. 19-38.

[27] Beletsky, Cigré, working group A2.12, Session paper "Insulation problems in power transformers for HVDC transmissions". 1976, pp. 1-9.

[28] M. Khanali, "Effects of Distorted Voltages on the Performance of Renewable Energy Plant Transformers," UWSpace, 2017. [Online]. Available: http://hdl.handle.net/10012/12368

[29] T. L. Koltunowicz, "Accelerated Insulation Aging Due to Thermal and Electrical Stresses in Future Power Grids," Boxpress, 2014. [Online]. Available: https://books.google.se/books?id=PnxOrgEACAAJ

[30] T. Koltunowicz, A. Cavallini, D. Djairam, G. C. Montanari, and J. Smit, "The influence of square voltage waveforms on transformer insulation break down voltage," in 2011 Annual Report Conference on Electrical Insulation and Dielectric Phenomena, 16-19 Oct. 2011 2011, pp. 48-51, doi: 10.1109/CEIDP.2011.6232593.

[31] M. Khanali and S. Jayaram, "Partial discharge activities in transformer insulation under steep front voltages," in Electrical Insulation and Dielectric Phenomena (CEIDP), 2014 IEEE Conference on, 2014: IEEE, pp. 204-207.

[32] R. C. Kiiza, M. G. Niasar, R. Nikjoo, X. Wang, and H. Edin, "Change in partial discharge activity as related to degradation level in oil-impregnated paper insulation: effect of high voltage impulses," IEEE Transactions on Dielectrics and Electrical Insulation, vol. 21, no. 3, pp. 12431250, 2014, doi: 10.1109/TDEI.2014.6832271.

[33] P. H. Morshuis, "Degradation of solid dielectrics due to internal partial discharge: some thoughts on progress made and where to go now," IEEE Transactions on Dielectrics and Electrical Insulation, vol. 12, no. 5, pp. 905-913, 2005. 
[34] M. A. Bahmani and T. Thiringer, "Accurate Evaluation of Leakage Inductance in HighFrequency Transformers Using an Improved Frequency-Dependent Expression," IEEE Transactions on Power Electronics, vol. 30, no. 10, pp. 5738-5745, 2015, doi: 10.1109/TPEL.2014.2371057.

[35] E. Agheb, M. A. Bahmani, H. K. Høidalen, and T. Thiringer, "Core loss behavior in high frequency high power transformers -II: Arbitrary excitation," Journal of Renewable and Sustainable Energy, vol. 033113, no. 4, 2012. [Online]. Available: https://doi.org/10.1063/1.4727917.

[36] S. A. K. S.V. Kulkarni, "Transformer Engineering Design, Technology, and Diagnostics". CRC Press is an imprint of Taylor \& Francis Group, an Informa business, 2013.

[37] Z. Ouyang, O. C. Thomsen, and M. A. E. Andersen, "The analysis and comparison of leakage inductance in different winding arrangements for planar transformer," in 2009 International Conference on Power Electronics and Drive Systems (PEDS), 2-5 Nov. 2009 2009, pp. 11431148, doi: 10.1109/PEDS.2009.5385844.

[38] J. Li, C. Hu, and X. Pang, "Analysis of the leakage inductance of planar transformer," in 2009 9th International Conference on Electronic Measurement \& Instruments, 16-19 Aug. 2009 2009, pp. 1-273-1-276, doi: 10.1109/ICEMI.2009.5274877.

[39] A. Boyajian, "Leakage Reactance of Irregular Distributions of Transformer Windings by the Method of Double Fourier Series [includes discussion]," Transactions of the American Institute of Electrical Engineers. Part III: Power Apparatus and Systems, vol. 73, no. 2, pp. 1078-1086, 1954, doi: 10.1109/AIEEPAS.1954.4498933.

[40] L. Rabins, "Transformer reactance calculations with digital computers," Transactions of the American Institute of Electrical Engineers, Part I: Communication and Electronics, vol. 75, no. 3, pp. 261-267, 1956, doi: 10.1109/TCE.1956.6372526.

[41] C. R. Sullivan, "Optimal choice for number of strands in a litz-wire transformer winding," IEEE Transactions on Power Electronics, vol. 14, no. 2, pp. 283-291, 1999, doi: 10.1109/63.750181.

[42] F. Tourkhani and P. Viarouge, "Accurate analytical model of winding losses in round Litz wire windings," IEEE Transactions on Magnetics, vol. 37, no. 1, pp. 538-543, 2001, doi: 10.1109/20.914375.

[43] Z. Ouyang, J. Zhang, and W. G. Hurley, "Calculation of Leakage Inductance for High-Frequency Transformers," IEEE Transactions on Power Electronics, vol. 30, no. 10, pp. 5769-5775, 2015, doi: 10.1109/TPEL.2014.2382175.

[44] K. Zhang et al., "Accurate Calculation and Sensitivity Analysis of Leakage Inductance of HighFrequency Transformer With Litz Wire Winding," IEEE Transactions on Power Electronics, vol. 35, no. 4, pp. 3951-3962, 2020, doi: 10.1109/TPEL.2019.2936523.

[45] P. L. Dowell, "Effects of eddy currents in transformer windings," Proceedings of the Institution of Electrical Engineers, vol. 113, no. 8, pp. 1387-1394, 1966, doi: 10.1049/piee.1966.0236.

[46] J. A. Ferreira, "Analytical computation of AC resistance of round and rectangular litz wire windings," IEE Proceedings B - Electric Power Applications, vol. 139, no. 1, pp. 21-25, 1992, doi: 10.1049/ip-b.1992.0003.

[47] J. A. Ferreira, "Improved analytical modeling of conductive losses in magnetic components," IEEE Transactions on Power Electronics, vol. 9, no. 1, pp. 127-131, 1994, doi: 10.1109/63.285503.

[48] M. Mogorovic and D. Dujic, "100 kW, 10 kHz Medium-Frequency Transformer Design Optimization and Experimental Verification," IEEE Transactions on Power Electronics, vol. 34, no. 2, pp. 1696-1708, 2019, doi: 10.1109/TPEL.2018.2835564.

[49] K. Karsai, D. Kerényi, and L. Kiss, Large Power Transformers. Elsevier, 1987.

[50] M. Kharezy and T. Thiringer, "Challenges with the design of cost-effective series DC collection network for offshore wind-farms," presented at the 17th Wind Integration Workshop on Large scale Integration of Wind Power into Power Systems as well as on Transmission Networks for Offshore Wind Power Plants, Stockholm, Sweden, 2018.

[51] M. Kharezy, H. R. Mirzaei, J. Rastamo, M. Svensson, Y. Serdyuk, and T. Thiringer, "Performance of Insulation of DC/DC Converter Transformer for Offshore Wind Power 
Applications," presented at the CEIDP2020, Conference on Electrical Insulation and Dielectric Phenomena, NJ, USA, 2020.

[52] Cigré working group A2/D1.41, "Technical Brochure 646: HVDC transformer insulation: Oil conductivity". 2016, pp. 1-93.

[53] M. A. Bahmani, T. Thiringer, and M. Kharezy, "Design Methodology and Optimization of a Medium-Frequency Transformer for High-Power DC-DC Applications," IEEE Transactions on Industry Applications, vol. 52, no. 5, pp. 4225-4233, 2016, doi: 10.1109/TIA.2016.2582825.

[54] C. R. Sullivan, "Computationally efficient winding loss calculation with multiple windings, arbitrary waveforms, and two-dimensional or three-dimensional field geometry," IEEE Transactions on Power Electronics, vol. 16, no. 1, pp. 142-150, 2001, doi: 10.1109/63.903999.

[55] M. A. Bahmani, T. Thiringer, and H. Ortega, "An Accurate Pseudoempirical Model of Winding Loss Calculation in HF Foil and Round Conductors in Switchmode Magnetics," IEEE Transactions on Power Electronics, vol. 29, no. 8, pp. 4231-4246, 2014, doi: 10.1109/TPEL.2013.2292593.

[56] E. Agheb, "Medium Frequency High Power Transformers for All-DC Wind Parks," Norwegian University of Science and Technology, 2017. [Online]. Available:

http://hdl.handle.net/11250/2448642

[57] M. Eklund, N. Naphthenics, P. Jarman, and M. Edwall, "Transformer Oil Handbook". Nynas $A B, 2010$.

[58] K. L. R. Fritsche, F. Trautmann, T. Hammer, "Transformers for High Voltage Direct Current Transmission - Challenge, Technology and Development," (in DE), 2016. [Online]. Available: https://www.researchgate.net/publication/315657537.

[59] R. Asano and S. A. Page, "Reducing Environmental Impact and Improving Safety and Performance of Power Transformers With Natural Ester Dielectric Insulating Fluids," IEEE Transactions on Industry Applications, vol. 50, no. 1, pp. 134-141, 2014, doi: 10.1109/TIA.2013.2269532.

[60] I. Fofana, "50 years in the development of insulating liquids," IEEE Electrical Insulation Magazine, vol. 29, no. 5, pp. 13-25, 2013, doi: 10.1109/MEl.2013.6585853.

[61] T. G. Aakre, T. A. Ve, and $\varnothing$. L. Hestad, "Conductivity and permittivity of midel 7131: effect of temperature, moisture content, hydrostatic pressure and electric field," IEEE Transactions on Dielectrics and Electrical Insulation, vol. 23, no. 5, pp. 2957-2964, 2016, doi: 10.1109/TDEI.2016.7736858.

[62] P. J. Rumpelt, F., "Oil Impregnated Pressboard Barrier Systems Based on Ester Fluids for an Application in HVDC Insulation Systems," (in EN), MDPI Energies, 2017, Art no. 10122147. [Online]. Available: https://www.mdpi.com/1996-1073/10/12/2147\#cite.

[63] H. P. Moser, "Transformerboard". Weidmann AG, 1979.

[64] Cigré working group D1.12, "Technical Brochure 520: Material properties of solid HVDC Insulation systems". 2012, pp. 1-16.

[65] A. Küchler, "High Voltage Engineering: Fundamentals-Technology-Applications". Springer, 2017.

[66] M. Kharezy, M. Eslamian, and T. Thiringer, "Insulation Design of a Medium Frequency Power Transformer for a Cost-Effective Series High Voltage DC Collection Network of an Offshore Wind Farm," in Proceedings of the 21st International Symposium on High Voltage Engineering, Cham, B. Németh, Ed., 2020// 2020: Springer International Publishing, pp. 14061417.

[67] H. Okubo, T. Sakai, T. Furuyashiki, K. Takabayashi, and K. Kato, "HVDC electric field control by pressboard arrangement in oil-pressboard composite electrical insulation systems," in 2016 IEEE Conference on Electrical Insulation and Dielectric Phenomena (CEIDP), 16-19 Oct. 2016 2016, pp. 35-39, doi: 10.1109/CEIDP.2016.7785513.

[68] M. Liebschner, Interaktion von Ölspalten und fester Isolation in HVDC-Barrierensystemen. VDI-Verlag, 2009. 
[69] K. B. T. Gabler, J. Speck, R. Fritsche and S. Großmann, "Experimental Validation of the Charge Carrier-based Modelling of Oil-Paper Insulations at High DC Voltage Stress," Conference: 20th International Symposium on High Voltage EngineeringAt: Buenos Aires, Argentina, 2017.

[70] K. Backhaus, J. Speck, M. Hering, S. Großmann, and R. Fritsche, "Nonlinear dielectric behaviour of insulating oil under HVDC stress as a result of ion drift," in 2014 ICHVE International Conference on High Voltage Engineering and Application, 8-11 Sept. 20142014 pp. 1-4, doi: 10.1109/ICHVE.2014.7035453.

[71] K. B. T. Gabler, T. Goetz, T. Linde, S. Grossmann and R. Fritsche, "Investigation on the breakdown behaviour of oil-paper insulations at superimposed DC and lightning impulse voltage stress," Conference: VDE-Hochspannungstechnik 2018 - ETG-Fachtagung, Berlin, Germany, 2018.

[72] F. Schober, S. Harrer, A. Kuchler, F. Berger, W. Exner, and C. Krause, "Transient and steadystate dc behavior of oil-impregnated pressboard," IEEE Electrical Insulation Magazine, vol. 32, no. 4, pp. 8-14, 2016, doi: 10.1109/MEI.2016.7528985.

[73] F. Vahidi, S. Tenbohlen, M. Rösner, C. Perrier, and H. Fink, "Influence of electrode material on conductivity measurements under DC stresses," in 2014 IEEE 18th International Conference on Dielectric Liquids (ICDL), 29 June-3 July 2014 2014, pp. 1-5, doi: 10.1109/ICDL.2014.6893133.

[74] T. Gabler, K. Backhaus, T. Götz, S. Großmann, and R. Fritsche, "Effect of the Non-Linear Electric Conductivity of Mineral Insulating Oil on the Dielectric Strength at High DC Voltage Stress," in 2018 IEEE Conference on Electrical Insulation and Dielectric Phenomena (CEIDP), 21-24 Oct. 2018 2018, pp. 54-57, doi: 10.1109/CEIDP.2018.8544845.

[75] IEC 62631-3-1: Determination of resistive properties (DC methods) - Volume resistance and volume resistivity - General method, ICS 17.220.99; 29.035.01, 2016.

[76] Küchler, Cigré, Study Comittee D1, Session paper "Evaluation of Conductivities and Dielectric Properties for Highly Stressed HVDC Insulating Materials". 2010, pp. 1-10.

[77] M. Lisowski and R. Kacprzyk, "Changes proposed for the IEC 60093 Standard concerning measurements of the volume and surface resistivities of electrical insulating materials," IEEE Transactions on Dielectrics and Electrical Insulation, vol. 13, no. 1, pp. 139-145, 2006, doi: 10.1109/TDEI.2006.1593412.

[78] ASTM D 257-14: Standard test methods for dc resistance or conductance of insulating materials, ASTM, 2014.

[79] Küchler, Cigré, Study Comittee A2, Session paper "Condition Assessment of Aged transformer Bushing Insulation". 2006, pp. 1-10.

[80] M. Eslamian, M. Kharezy, and T. Thiringer, "Calculation of the Leakage Inductance of Medium Frequency Transformers with Rectangular-Shaped Windings using an Accurate Analytical Method," in 2019 21st European Conference on Power Electronics and Applications (EPE '19 ECCE Europe), 3-5 Sept. 2019 2019, pp. P.1-P.10, doi: 10.23919/EPE.2019.8915036.

[81] W. Shen, "Design of High-density Transformers for High-frequency High-power Converters," Virginia Polytechnic Institute and State University, 2006. [Online]. Available: https://books.google.se/books?id=XG8CkAEACAAJ

[82] C. P. Steinmetz, "On the Law of Hysteresis," Transactions of the American Institute of Electrical Engineers, vol. IX, no. 1, pp. 1-64, 1892, doi: 10.1109/T-AIEE.1892.5570437.

[83] TDK. "SIFERRIT material N87." https://www.tdkelectronics.tdk.com/download/528882/71e02c7b9384de1331b3f625ce4b2123/pdf-n87.pdf (accessed 25-08-2020).

[84] Hitachi. "FINEMET F3CC Series Cut Cores." https://www.hitachimetals.co.jp/e/products/elec/tel/pdf/hl-fm34.pdf (accessed 25-08-2020).

[85] R. W. A. A. D. Doncker, D. M. Divan, and M. H. Kheraluwala, "A three-phase soft-switched high-power-density DC/DC converter for high-power applications," IEEE Transactions on Industry Applications, vol. 27, no. 1, pp. 63-73, 1991, doi: 10.1109/28.67533. 
[86] Vonroll. "Winding Wires and Litz Wires."

https://www.vonroll.com/userfiles/downloads/1576761040530073/2.01.1 Wire EN.pdf (accessed 25-08-2020).

[87] O. Moreau, L. Popiel, and J. L. Pages, "Proximity losses computation with a 2D complex permeability modelling," IEEE Transactions on Magnetics, vol. 34, no. 5, pp. 3616-3619, 1998, doi: 10.1109/20.717854.

[88] J. Gyselinck and P. Dular, "Frequency-domain homogenization of bundles of wires in 2-D magnetodynamic FE calculations," IEEE Transactions on Magnetics, vol. 41, no. 5, pp. 14161419, 2005, doi: 10.1109/TMAG.2005.844534.

[89] D. Meeker. "Continuum Representation of Wound Coils via an Equivalent Foil Approach." www.femm.info (accessed 25-08-2020).

[90] IEC 60076-3: Power transformers - Insulation levels, dielectric tests and external clearances in air 978-2-83220-830-4, 2013.

[91] IEC 61251: Electrical insulating materials and systems - A.C. voltage endurance evaluation, ICS 17.220.99; 29.035.01, 2015.

[92] G. C. Stone, R. G. v. Heeswijk, and R. Bartnikas, "Investigation of the effect of repetitive voltage surges on epoxy insulation," IEEE Transactions on Energy Conversion, vol. 7, no. 4, pp. 754-760, 1992, doi: 10.1109/60.182659.

[93] D. R. Johnston, J. T. LaForte, P. E. Podhorez, and H. N. Galpern, "Frequency Acceleration of Voltage Endurance," IEEE Transactions on Electrical Insulation, vol. El-14, no. 3, pp. 121-126, 1979, doi: 10.1109/TEI.1979.298211.

[94] IEC 60034-18-41: Rotating electrical machines - Part 18-41: Partial discharge free electrical insulation systems (Type I) used in rotating electrical machines fed from voltage converters Qualification and quality control tests, ICS 29.160, 2014.

[95] IEC 60156: Insulating liquides - Determination of breakdown voltage at power frequency Test method, 1995.

[96] IEC 60851-5: Winding wires - Test methods - Part 5: Electrical properties, ICS 29.060.10, 2008.

[97] S. M. Hasheminezhad, E. Ildstad, and A. Nysveen, "Breakdown strength of solid|solid interface," in 2010 10th IEEE International Conference on Solid Dielectrics, 4-9 July 2010 2010, pp. 1-4, doi: 10.1109/ICSD.2010.5568229.

[98] IEC 60243-1: Electric strength of insulating materials -Test methods - Part 1: Tests at power frequencies, ICS 17.220.99; 29.035.01, 2013.

[99] ASTM D149-97a: Standard test method for dielectric breakdown voltage and dielectric strength of solid electrical insulating materials at commercial power frequencies, 2004.

[100] G. Steeves. "'Vapor phase transformer drying - Part I," Transformer Magazine, Volume3, Issue 2, April 2016." www.transformers-magazine.com (accessed 25-08-2020).

[101] IEC 60641-2: Pressboard and presspaper for electrical purposes - Part 2: Methods of tests, ICS 17.220.99, 2004.

[102] F. Vahidi, S. Tenbohlen, M. Rösner, C. Perrier, and H. Fink, "The investigation of the temperature and electric field dependency of mineral oil electrical conductivity," Conference: VDE-Hochspannungstechnik 2013 - ETG-Fachtagung, Dresden, Germany, 2013.

[103] Y. Li, K. Zhou, G. Zhu, M. Li, S. Li, and J. Zhang, "Study on the Influence of Temperature, Moisture and Electric Field on the Electrical Conductivity of Oil-Impregnated Pressboard," (in EN), MDPI Energies, 2019, Art no. 12163136 [Online]. Available:

https://www.mdpi.com/1996-1073/12/16/3136\#cite.

[104] P. Rumpelt and F. Jenau, "Investigation on DC conductivity of alternative insulating oils as an application for HVDC converter transformers," in 2017 IEEE 19th International Conference on Dielectric Liquids (ICDL), 25-29 June 2017 2017, pp. 1-6, doi: 10.1109/ICDL.2017.8124607.

[105] C. Daran-Daneau, D. É, M. F. Fréchette, and S. Savoie, "Influence of the surrounding medium on the dielectric strength measurement of LLDPE/clay nanocomposites," in 2012 IEEE International Symposium on Electrical Insulation, 10-13 June 2012 2012, pp. 654-658, doi: 10.1109/ELINSL.2012.6251553. 
[106] Y. Ohki, "News from Japan," IEEE Electrical Insulation Magazine, vol. 29, no. 6, pp. 71-74, 2013, doi: 10.1109/MEI.2013.6648759.

[107] M. Mogorovic and D. Dujic, "Sensitivity Analysis of Medium-Frequency Transformer Designs for Solid-State Transformers," IEEE Transactions on Power Electronics, vol. 34, no. 9, pp. 8356-8367, 2019, doi: 10.1109/TPEL.2018.2883390.

[108] D. Meeker. "Finite element method magnetics, Version 4.2." www.femm.info (accessed 2508-2020).

[109] H. Yongtao, W. Eberle, and L. Yan-Fei, "New measurement methods to characterize transformer core loss and copper loss in high frequency switching mode power supplies," in 2004 IEEE 35th Annual Power Electronics Specialists Conference (IEEE Cat. No.04CH37551), 20-25 June 2004 2004, vol. 2, pp. 1695-1701 Vol.2, doi: 10.1109/PESC.2004.1355681.

[110] O. Guillén Sentís, "Feasibility of sea based wind park," Chalmers University of Technology, 2020. [Online]. Available: https://hdl.handle.net/20.500.12380/301473

[111] T. C. Estate. "The Crown Estate, Guide to an offshore wind farm " https://www.thecrownestate.co.uk/media/2860/guide-to-offshore-wind-farm-2019.pdf (accessed 25-08-2020).

[112] NorthSeaGrid. "Offshore electricity grid implementation in the north sea, Technical report." http://northseagrid.info/sites/default/files/NorthSeaGrid Final Report.pdf (accessed 25-082020).

[113] Entsoe. "Offshore Transmission Technology " https://eepublicdownloads.blob.core.windows.net/public-cdn-container/cleandocuments/pre2015/publications/entsoe/SDC/European offshore grid Offshore Technology - FINALversion.pdf (accessed 25-08-2020).

[114] P. Härtel, T. K. Vrana, T. Hennig, M. v. Bonin, E. J. Wiggelinkhuizen, and F. D. J. Nieuwenhout, "Review of investment model cost parameters for vsc hvdc transmission infrastructure," Elsevier Electric Power Systems Research, vol. 151, pp. 419-431, 2017. [Online]. Available: https://doi.org/10.1016/j.epsr.2017.06.008.

[115] DNV.GL. "66 kV Systems for Offshore Wind Farms " https://www.tennet.eu/fileadmin/user upload/Our Grid/Offshore Netherlands/Consultatie proces net op zee/Technical Topics/4 T1. Enclosure $\mathrm{nr} 1 \mathrm{~b}$ 66 kV systems for Offshore Wind Farms by DNV GL.pdf (accessed 25-08-2020).

[116] IEC EN 60071-1:2006 "Insulation co-ordination - Part 1: Definitions, principles and rules, 2006.

[117] Pfisterer. "Cable systems, cable fittings for high voltage networks." https://www.pfisterer.com/fileadmin/pfisterer/downloads en/CableSystemHV-CT-EN.pdf (accessed 25-08-2020).

[118] B. Dolata, H. Borsi, and E. Gockenbach, "Comparison of Electric and Dielectric Properties of Ester Fluids with Mineral Based Transformer Oil," presented at the Proceedings of the 15st International Symposium on High Voltage Engineering, Ljubljana, Slovenia, 2007.

[119] M. Lashbrook, A. Gyore, and R. Martin, "A review of the fundamental dielectric characteristics of ester-based dielectric liquids," Elsevier Science Direct Procedia Engineering, vol. 202, pp. 121-129, 2017. [Online]. Available: https://doi.org/10.1016/i.proeng.2017.09.699. 